
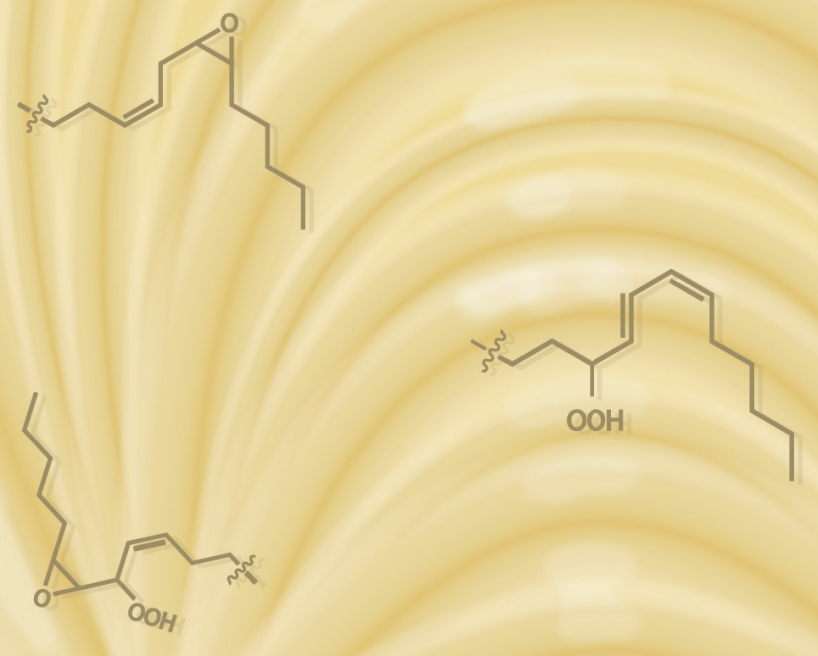

Mechanism-based markers for early assessment of lipid oxidation in mayonnaise by magnetic resonance spectroscopy
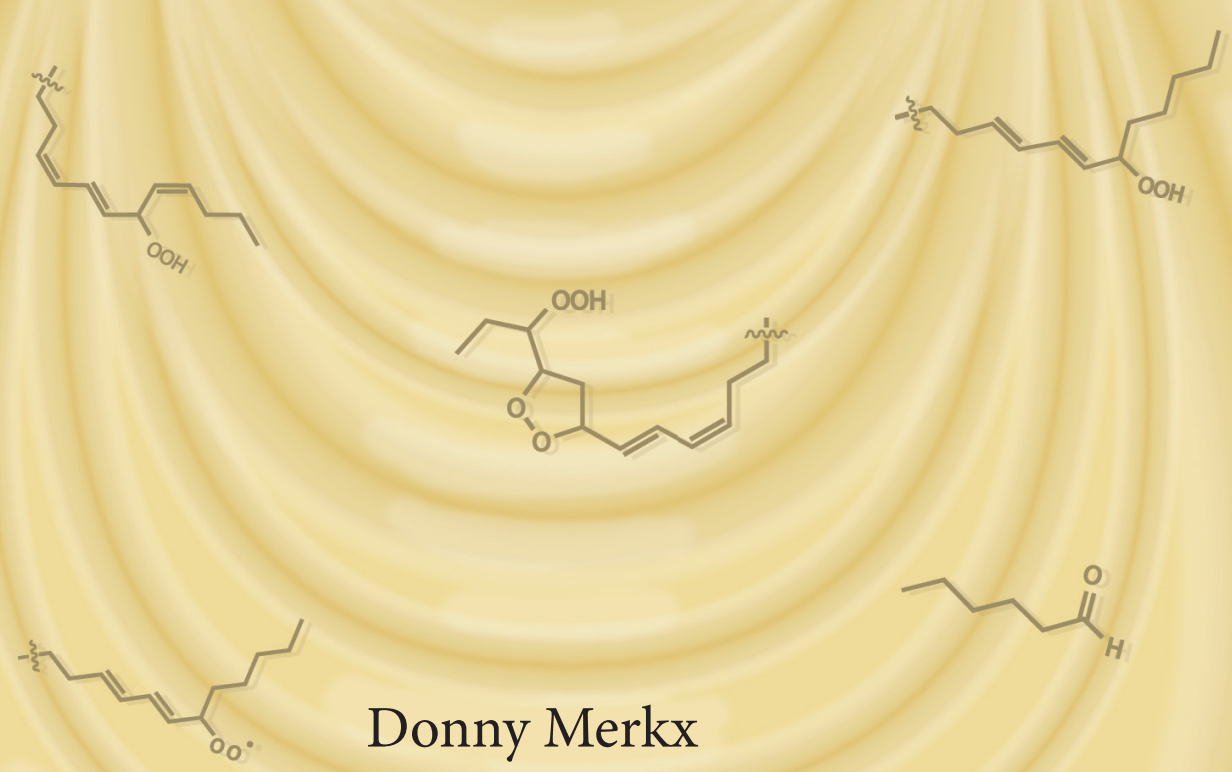

Donny Merkx 


\section{Propositions}

1. NMR is the most versatile tool to obtain broad comprehension of lipid oxidation. (this thesis)

2. Understanding $\mathrm{LOOH}$ kinetics is understanding lipid oxidation. (this thesis)

3. Gatekeeping (Barzilai-Nahon, 2009) of analytical methods withholds them from the wider scientif ic community and severely impedes the speed of scientific progress .

4. Importance of soft skills is undervalued in academia.

5. Physical fitness should be the cornerstone of education to improve student's cognitive functions (Bass, 2013).

6. Taxing capital income more progressively and aggressively (Bastani \& Waldenström, 2020 ) is an effective way to reduce wealth disparity.

Propositions belonging to the thesis, entitled

Mechanism-based markers for early assessment of lipid oxidation in mayonnaise by magnetic resonance spectroscopy

Donny Merkx

Wageningen, 13 October 2021 

Mechanism-based markers for early assessment of lipid oxidation in mayonnaise by magnetic resonance spectroscopy

\author{
Donny Merkx
}




\section{Thesis committee}

\section{Promotor}

Prof. Dr John P.M. van Duynhoven

Special Professor Magnetic Resonance in relation to Food

Wageningen University \& Research

\section{Co-promotor}

Dr Marie Hennebelle

Assistant professor, Food Chemistry

Wageningen University \& Research

\section{Other members}

Prof. Dr CGPH (Karin) Schroën, Wageningen University \& Research

Prof. Dr Charlotte Jacobsen, Technical University of Denmark, Kongens Lyngby, Denmark Dr Mickaël Laguerre, Givaudan, Avignon, France

Prof. Dr Hanne C.S. Bertram, Aarhus University, Aarhus, Denmark

This research was conducted under the auspices of the Graduate School VLAG (Advanced studies in Food Technology, Agrobiotechnology, Nutrition and Health Sciences). 


\title{
Mechanism-based markers for early assessment of lipid oxidation in mayonnaise by magnetic resonance spectroscopy
}

\author{
Donny Merkx
}

\section{Thesis}

submitted in fulfilment of the requirements for the degree of doctor at Wageningen University

by the authority of the Rector Magnificus,

Prof. Dr. A.P.J. Mol,

in the presence of the

Thesis Committee appointed by the Academic Board

to be defended in public

on Wednesday 13 October 2021

at 4 p.m. in the Aula 
Donny Merkx

Mechanism-based markers for early assessment of lipid oxidation in mayonnaise by magnetic resonance spectroscopy

204 pages

$\mathrm{PhD}$ thesis, Wageningen University, Wageningen, the Netherlands (2021)

With references, with summary in English

ISBN: 978-94-6395-900-1

DOI: https://doi.org/10.18174/550169 


\section{Table of content}

Chapter 1

Chapter 2

Appendix - Chapter 2

Chapter 3

Chapter 4

Chapter 5

Chapter 6

Chapter 7
General introduction

7

Rapid quantitative profiling of lipid oxidation products

27

in a food emulsion by ${ }^{1} \mathrm{H}$ NMR

Quantitative ${ }^{1} \mathrm{H}$ NMR analysis of food lipid

hydroperoxides and aldehydes

Lipid epoxide quantification in a food emulsion by ${ }^{1} \mathrm{H}-$

67

${ }^{13}$ C HSQC NMR spectroscopy

Evaluation of PBN spin-trapped radicals as early

95

markers of lipid oxidation in mayonnaise

Quantitative and predictive modelling of lipid

123

oxidation in mayonnaise

${ }^{31} \mathrm{P}$ NMR assessment of the phosvitin-iron complex in mayonnaise

General discussion

Summary

189

Acknowledgements

193

About the author

199 


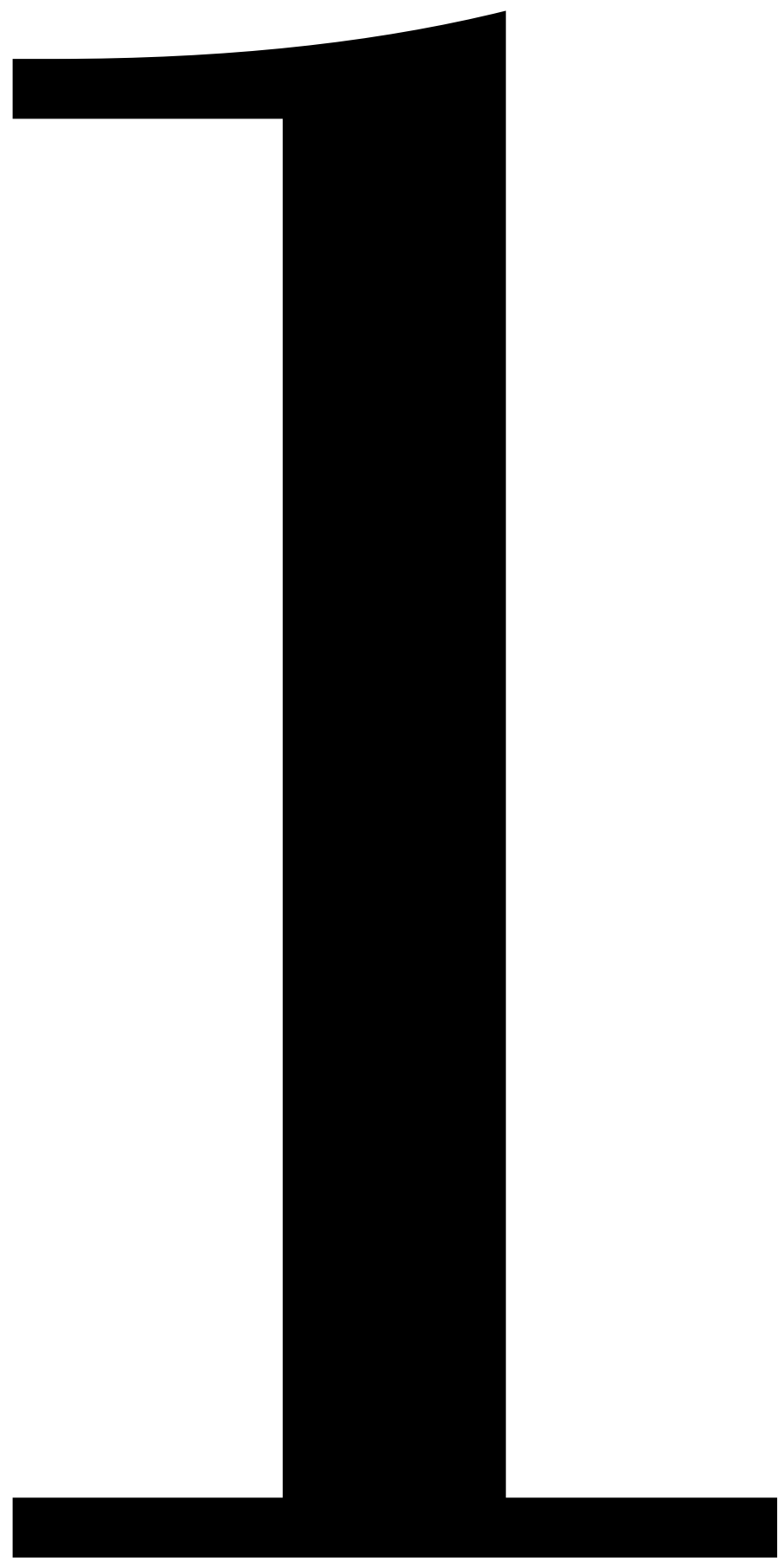


Chapter 1

General Introduction 


\subsection{Introduction}

Lipid oxidation is a major concern in the food industry as it is one of the main contributors to the limited shelf life of lipid-based food products. This is a particular concern for food emulsions with high amounts of unsaturated fats, such as mayonnaise. In the food industry, accelerated shelf-life tests (ASLTs) are often applied to assess the oxidative stability of different formulations. The development of new technologies to combat lipid oxidation is hampered by the lack of rapid shelf-life evaluation tools that provide mechanistical insights. In this thesis, we developed rapid and quantitative NMR and ESR methods that cover major lipid oxidation initiators and intermediates, both in the oil as well as the water phase of food emulsion. Aided by semi-empirical models, these methods will be utilised to shorten shelflife testing.

\subsubsection{Limited stability of natural mayonnaise}

Fats are one of three macronutrients, next to proteins and carbohydrates. This means that they are essential to the diet in large quantities on a daily basis. The two main sources of fat in a healthy diet are animal-based (meat, milk, eggs) and plant-based (seeds, nuts, oils, dressings). In this thesis, the focus will be on the latter, specifically the oil from pressed seeds. Often, these oils are not consumed as such in large quantities, but rather in emulsions. An emulsion is a dispersion of two immiscible liquids, such as water and oil, stabilised by surface-active molecules that lower the surface tension (i.e., surfactants). Typical food emulsions are either water-in-oil dispersions such as margarine, or oil-in-water dispersions such as salad dressing and mayonnaise. The latter, mayonnaise, has a particularly big market size, worth 10.3 billion dollars in 2018 and is projected to expand to 10.3 billion dollars by 2024. It is therefore of considerable interest for the industry. The main ingredients of mayonnaise, are, by order of quantity: vegetable oil, water, egg yolk, vinegar and salt (Figure 1.1). Often, flavourings such as sugar and mustard, amongst others, are added as well. Mayonnaise is considered a highfat emulsion, with a minimum amount of vegetable oil of $70 \% \mathrm{w} / \mathrm{w}$ and $65 \% \mathrm{w} / \mathrm{w}$ in Europe and the United States, respectively. Furthermore, in Europe, mayonnaise cannot be called as such if egg yolk is not present in at least $5 \% \mathrm{w} / \mathrm{w}$. Egg yolk contains phospholipids and proteins that both act as emulsifiers and surfactants. Without these emulsifiers, the emulsion will separate rapidly into a bilayer system. Egg yolk does bring a significant amount of iron to the emulsion system, which may have a detrimental effect on the chemical stability of unsaturated oils. 


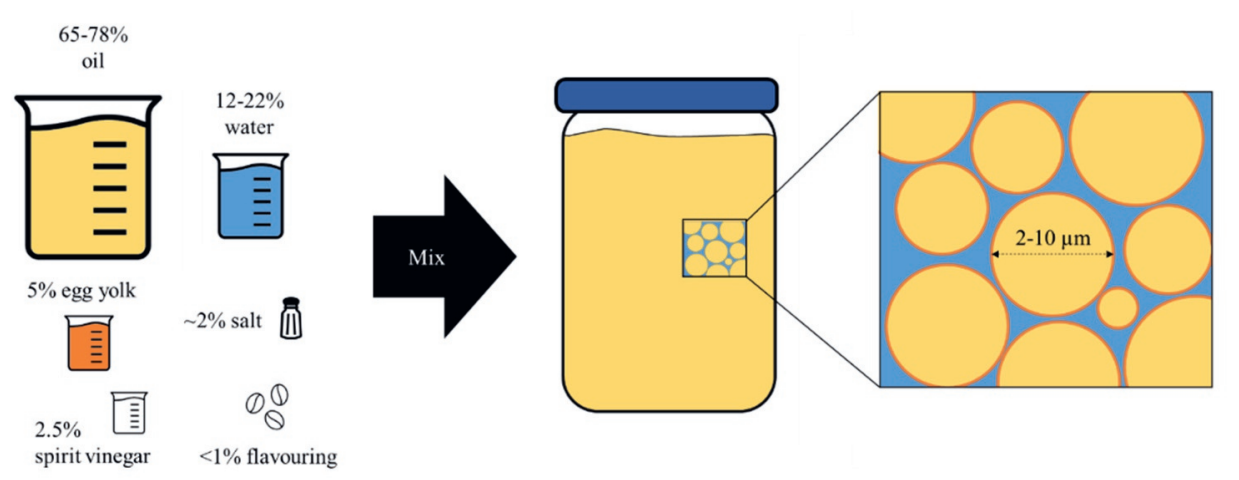

Figure 1.1. Schematic overview of mayonnaise ingredients and the physical structure of this high-fat oil-in-water emulsion.

To ensure the quality and safety of emulsions, it is essential to assess the physical, microbiological, and chemical stability of such a product. For mayonnaise, the physical and microbiological stabilities can be readily maintained for a long period of time with current technologies. Chemical deterioration, however, is still a major issue. The main process that is responsible for this deterioration is called lipid oxidation. Current technologies to combat lipid oxidation in food emulsions involve chilled supply chains and/or the use of artificial (Elabelled) antioxidants. There is, however, an increasing demand from consumers for more sustainable (ambient supply chain) and natural (clean label) products. Even though lipid oxidation is responsible for most of the chemical deterioration, it is still a slow process if it is compared to typical industrial product development cycles. To put it into context, a mayonnaise without any oxidative measures, such as adding antioxidants, is still very well edible (so low off-flavours) after one month under consumer relevant conditions (fridge, closed lid). One can imagine that when developing new products with novel ingredients to combat lipid oxidation, this timescale is too long. Therefore, when developing new routes, accelerated shelf-life tests (ASLTs) are often employed. In these ASLTs, stimulation of oxidative mechanisms can be achieved in different ways (Calligaris, Manzocco, Anese, \& Nicoli, 2016; Manzocco, Calligaris, Anese, \& Nicoli, 2016). One can add pro-oxidants such as transition metals (Berton, Ropers, Bertrand, Viau, \& Genot, 2012; Gutteridge, Richmond, \& Halliwell, 1979; Schröder, Sprakel, Boerkamp, Schroën, \& Berton-Carabin, 2019), manipulate the amount of oxygen content and its state (Johnson \& Decker, 2015) or increase the storage temperature (Gómez-Alonso, Mancebo-Campos, Desamparados Salvador, \& Fregapane, 2004; Ragnarsson \& Labuza, 1977). The latter is the most popular option due to its convenience and low cost, as the only thing that is required is an oven. The major downside of using temperature as a stimulus of the oxidation process is that it not only increases the speed of the reaction, but it can also change the type of oxidation products that are formed. Increasing temperature will also influence the physical structure of the emulsion. It can lead 
to evaporation of the continuous water layer and/or destabilization of the dispersed droplets. Nonetheless, it is the best option in assessing lipid oxidation if time is a limited commodity. The assessment of shelf-life tests is typically done by assessing the molecules that are responsible for the off-flavour and/or by one-dimensional methods such as oxygen consumption. ASLTs reduce the time to assess the oxidative stability from multiple months to multiple weeks, but this time-gain of ASLTs can be compromised by having different mechanisms at play than under consumer-relevant conditions. Current assessment methods cannot diagnose such mechanistic modulations. Furthermore, current one-dimensional methods are labour-intensive and time-consuming, which strains rapid and cost-effective development of novel formulations. Therefore, there is a need for rapid quantitative tools that can help understanding lipid oxidation under different storage conditions.

\subsubsection{Lipid oxidation principles}

Before delving into the oxidative stability problems in mayonnaises, it is essential to explain the basic principles of lipid oxidation. Even though lipid oxidation in food emulsions is more complex than in bulk oil, the principles still apply. In both oil and mayonnaise, the bulk of lipids found are in triglyceride (triacylglycerol) form. Triglycerides are free fatty acids that are esterified to a glycerol backbone. The glycerol backbone is always the same, but these fatty acids can differ in two major ways. In edible oils, they are mainly 16 or 18 carbons long and the tail characteristics can be divided in three subclasses: saturated, mono-unsaturated and poly-unsaturated (Table 1.1). In saturated fatty acids, the tail is fully hydrogenated, which means that each carbon is sp3-hybridised and surrounded by hydrogen atoms. For the unsaturated fatty acids, one (mono) or more (poly) of the $\mathrm{C}-\mathrm{C}$ bonds are not fully hydrogenated, so they can form a double bond $\mathrm{C}=\mathrm{C}$. Due to this unsaturation, the carbon-tail is less rigid and does not crystallise that readily as compared to saturated fatty acids. This unsaturation does make the fatty acid susceptible for oxidation. Lipid oxidation, sometimes referred to as fat oxidation, is a chemical process in which oxygen reacts with the unsaturated moieties of the fatty acid chain. This process yields new molecules which are often undesired, such as aldehydes and epoxides. It can be stimulated by the presence of certain enzymes or light and are hence referred to as enzymatic oxidation and photo-oxidation. In food systems, however, the major problems stem from the autooxidation process, which can be interpreted as 'self-inflicted' oxidation. This is a process that only requires oxygen and unsaturated fatty acids, but can be catalysed by, for example, transition metals.

In its simplest form, lipid autooxidation is a free radical chain reaction that is traditionally divided in three phases: initiation, propagation, and termination. In the initiation phase, a free radical is formed by abstraction of a hydrogen atom from the alpha-position of the olefine (double bond), or referred to as allylic moiety in the fatty acid (LH) chain (Eq. 1.1). This 
initiation can be triggered spontaneously or by the presence of a radical initiator. In the next phase, the propagation, this free radical can react with oxygen (Eq. 1.2), forming a peroxyradical (LOO·) that can subsequently abstract a hydrogen from another $\mathrm{LH}$, forming a lipid radical $(\mathrm{L} \cdot)$ and hydroperoxide $(\mathrm{LOOH})$ in the process (Eq. 1.3). The oxidative susceptibility of a fatty acid chain primarily depends on the number of double bounds, and their positions regarding one another. When only a single double bond is present, as in oleic acid, the free radical that is present after the hydrogen abstraction at the allylic position can only be weakly stabilised by resonance. However, in both linoleic and alpha-linolenic acid, respectively two and three double bonds are present and are separated by only one $\mathrm{CH}_{2}$. This $\mathrm{CH}_{2}$ is on the so-called bisallylic position, and when a hydrogen is abstracted from this position, it can be very well stabilised by resonance. This resonance pushed the formation of the thermodynamically more favourable radical. In turn, this means that this radical is longer lived with an increased chance to react with oxygen in the next step. Thus, these polyunsaturated fatty acid chains are much more susceptible to lipid oxidation than their monounsaturated counterparts. This free radical chain reaction will slow down if the radicals start reacting with each other, which is called the termination phase (Eq. 1.4/1.5, amongst many other possible reactions). It is important to note that, even though these steps are postulated in order, all three process steps are continuously happening at the same time at different rates.

\section{Initiation}

$$
\mathrm{LH} \rightarrow \mathrm{L} \cdot+\mathrm{H} \cdot
$$

\section{Propagation}

$$
\begin{array}{cc}
\mathrm{L} \cdot+\mathrm{O}_{2} \rightarrow \mathrm{LOO} \cdot & \text { Eq. } 1.2 \\
\mathrm{LOO} \cdot+\mathrm{LH} \rightarrow \mathrm{LOOH}+\mathrm{L} \cdot & \text { Eq. } 1.3
\end{array}
$$

Termination

$$
\begin{array}{cr}
\mathrm{L} \cdot+\mathrm{L} \cdot \rightarrow \mathrm{LL} & \text { Eq. } 1.4 \\
\mathrm{~L} \cdot+\mathrm{LOO} \cdot \rightarrow \mathrm{LOOL} & \text { Eq. } 1.5
\end{array}
$$


Table 1.1 Overview of the main fatty acids that are present in edible oils, with their trivial names including common abbreviations, chemical structure, and relative reactivity. Allylic protons are coloured orange, bisallylic protons are coloured red, to illustrate their increased oxidative susceptibility. The fatty acid distribution on four of the most common oils in food products are displayed on the right of the table.

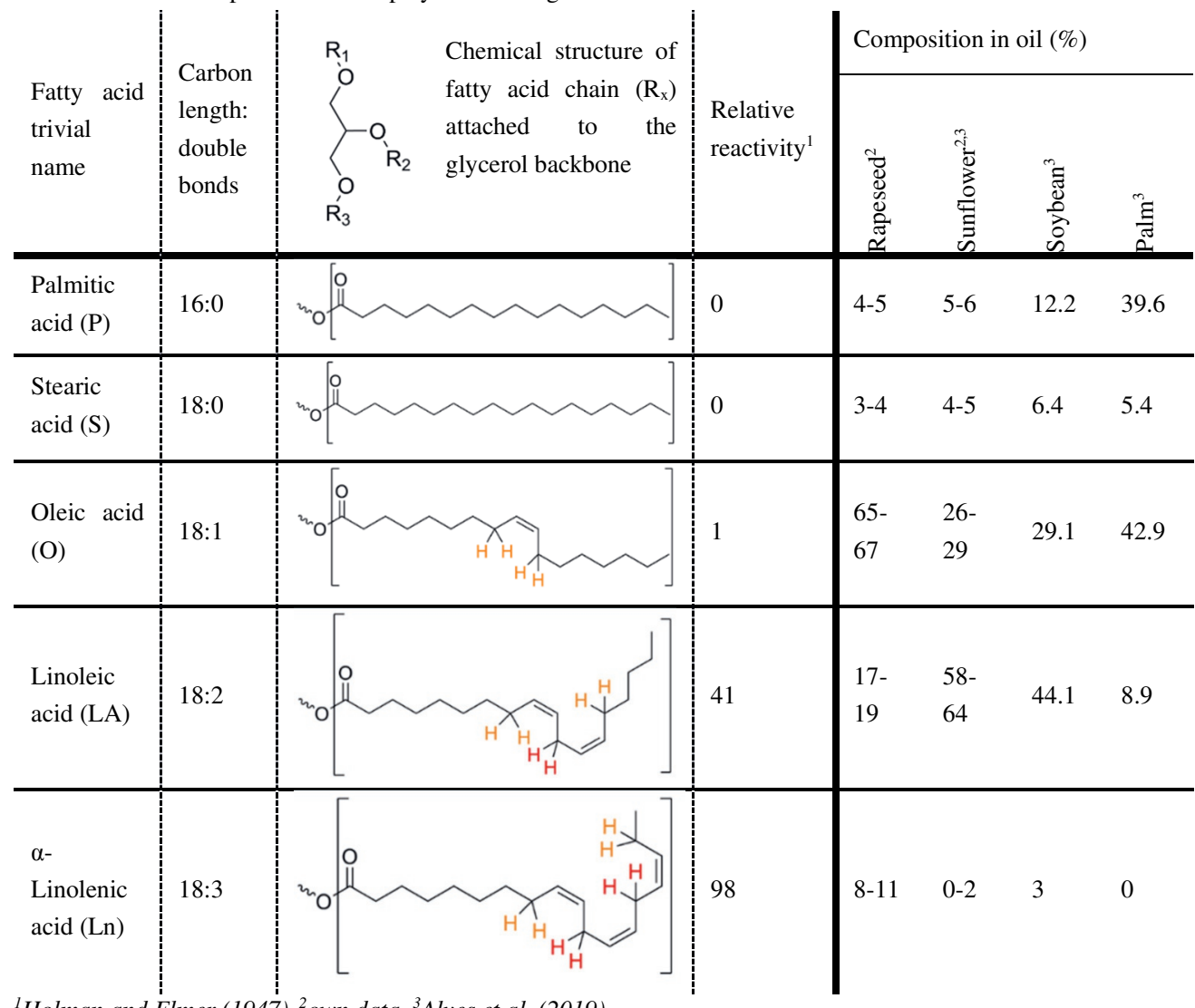

${ }^{1}$ Holman and Elmer (1947), ${ }^{2}$ own data, ${ }^{3}$ Alves et al. (2019)

This free radical three-phase representation is an oversimplification of the actual process. There is common consensus that lipid oxidation can be divided into two main oxidation phases. The generation of hydroperoxides is often referred to as 'primary oxidation', logically followed by 'secondary oxidation'. Secondary oxidation is used as an umbrella term that comprises of the reactions the formed hydroperoxides can undergo. Hydroperoxides can readily decompose into alkoxyl (LO·) radicals both thermally (Eq. 1.6) as well as via transition metal catalysis (Eq. 1.7), where in the same redox cycle also peroxyl (LOO·) are formed (Eq.1.8). 


\section{Radical generation from $\mathrm{LOOH}$}

$$
\begin{gathered}
\mathrm{LOOH} \rightarrow \mathrm{LO} \cdot+\cdot \mathrm{OH} \\
\mathrm{LOOH}+\mathrm{M}^{3+} \rightarrow \mathrm{LOO} \cdot+\mathrm{H} \cdot+\mathrm{M}^{2+} \\
\mathrm{LOOH}+\mathrm{M}^{2+} \rightarrow \mathrm{LO} \cdot+\mathrm{H} \cdot+\mathrm{M}^{3+}
\end{gathered}
$$

Eq. 1.6

Eq. 1.7

Eq. 1.8

The presence of primarily the alkoxyl radical kicks off a myriad of degradation mechanism (E.N. Frankel, 2014; Schaich, 2012), generating a very wide range of oxidation products such as aldehydes, alkanes, alkenes, epoxides, endoperoxides, alcohols, polymers, and combinations of these functionalities. For these secondary oxidation products, there is more than one chemical pathway imaginable, e.g., it has been reported that epoxides can be formed both from peroxyl (LOO·) as well as alkoxyl (LO·) radicals. Of the above listed secondary oxidation products, the aldehydes, epoxides, and polymers are one of the most common. To supply a brief overview of how these products can be formed, pathways that include the alkoxy-radical (LO) are described in Figure 1.2. Aldehydes can be readily formed via homolytic beta-cleavage, generating an alkane or alkene in the process. Epoxides can be formed via internal cyclization, in a reaction where the free radical is still able to further react. This free radical can then continue its regular propagation and thus form hydroperoxylepoxides and subsequently epoxy-aldehydes even. Polymers can be formed via a reaction of two free radicals on different fatty acid chains, effectively forming polytriglycerides in the process.

\subsubsection{Lipid oxidation in emulsions: the impact of transition metals}

In the previous section, a basic view on lipid oxidation in bulk oil has been described. When considering lipid oxidation in food emulsions such as mayonnaises, this process gets both more complex as well as more rapid. The two main reasons for this added complexity and increased susceptibility to lipid oxidation are transition metal catalysis and the physical reactive interface. Transitional metals are a group of elements that have a partly filled dorbital, allowing these elements to have different oxidation states with a variety of catalytic properties. In the context of lipid oxidation in food emulsions, iron and copper are the most relevant transition metals. Both iron and copper can be present in small traces in the oil and other ingredients. Iron, however, is introduced in significant amounts when using egg as emulsifier, since it is complexed with the phosphoprotein phosvitin. Since egg is a mandatory ingredient of mayonnaise, the emphasis in this thesis will be on the role of iron. 


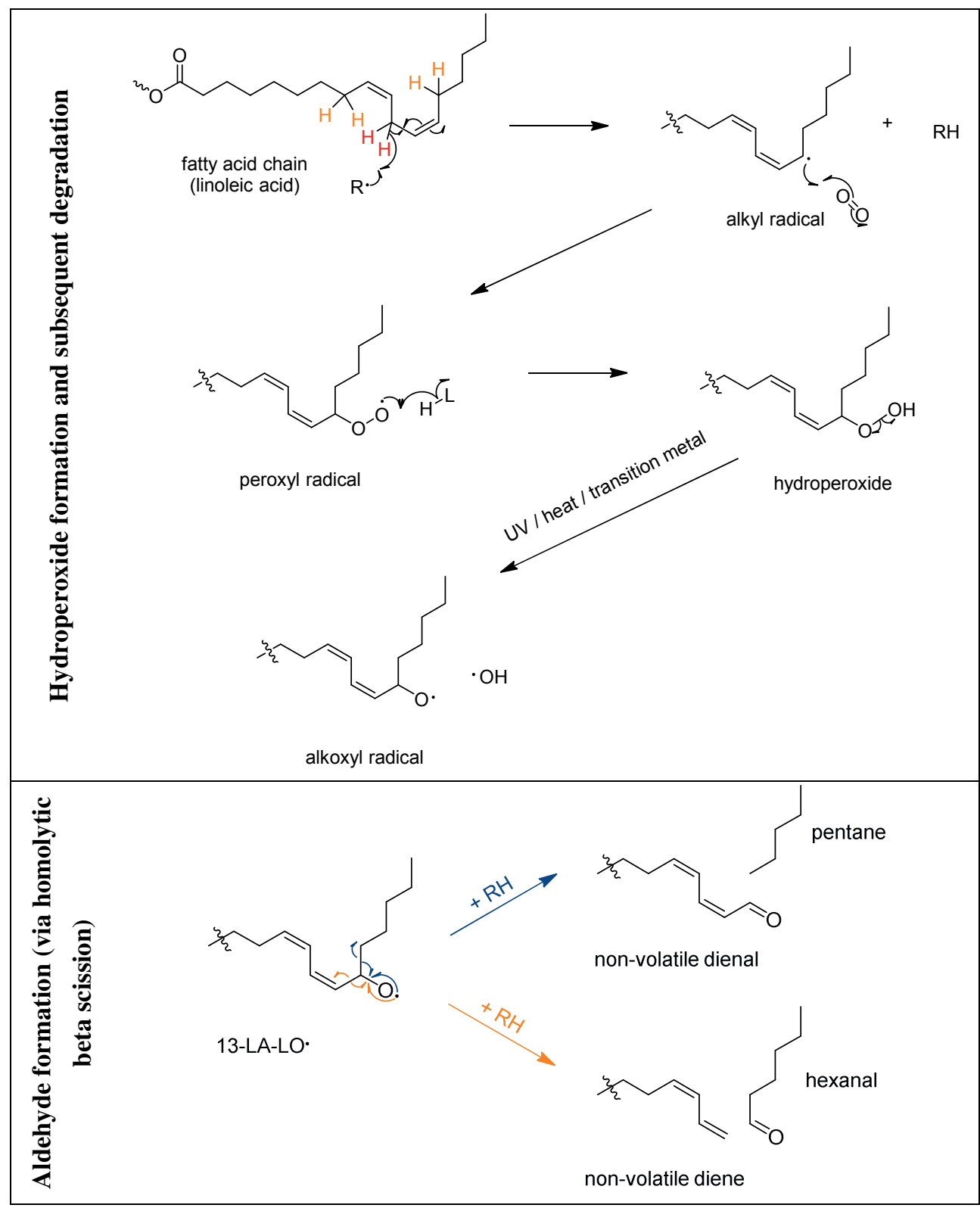




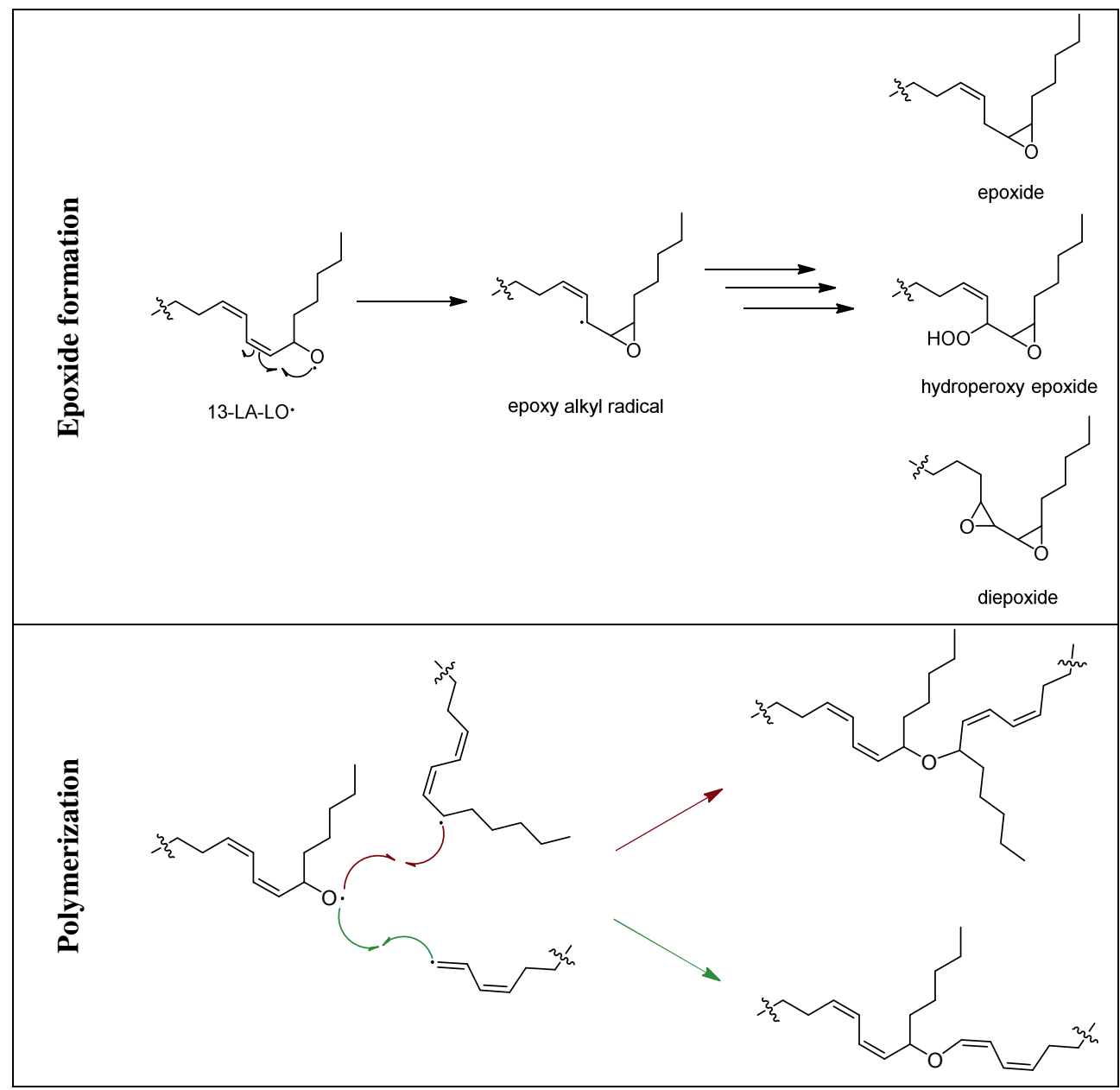

Figure 1.2. Overview of four of the main reaction mechanisms leading to common lipid oxidation products starting from the alkoxyl (LO·) radical. In this example, linoleic acid (LA/18:2) was used, where similar pathways can be postulated with the other fatty acids. The reactions show the basic principles, with many more possible outcomes not shown.

Iron ions have two dominant oxidation states, the ferrous and ferric ions, respectively $\mathrm{Fe}^{2+}$ and $\mathrm{Fe}^{3+}$. Both these oxidation states have a catalytic role in lipid oxidation and are constantly regenerated in a redox cycle (Eq. 1.7/1.8). In the traditional lipid oxidation mechanisms, the rate of the reaction is governed by the initiation step (Eq. 1.1), the abstraction of a hydrogen radical from the (bis)allylic position. When transition metals are present, the role of the hydroperoxides become more prevalent. Hydroperoxides can undergo iron-catalysed decomposition that will readily form more radicals and will boost the overall rate of oxidation. Even more so, when working with real oils and food emulsion, it is almost impossible to have material that is totally void of any hydroperoxides. In other words, in most 
systems, transition metals will have a substrate to reduce and oxidise from the beginning. Besides the presence of iron, it is also important how it physically reaches the reactive interphase, or how to prevent iron from reaching the interphase. For example, EDTA (Jacobsen et al., 2001) can act as a chelator that readily captures the iron, disallowing it to enter the redox cycle and slowing down lipid oxidation (Eq. 1.7/1.8). Considering these complexities, monitoring hydroperoxides will be essential to understand lipid oxidation in food emulsions.

\subsubsection{Lipid oxidation in emulsions: a colloidal game}

Compared to bulk oils, emulsions bring extra complexity and with that potentially extra oxidative reactivity. This increase is due to the chance for oxidation to take place in all phases of the emulsion (Jacobsen, Let, Nielsen, \& Meyer, 2008). As mentioned before, oil-in-water emulsions are colloidal systems in which the oil droplets, forming the dispersive phase, are suspended in the continuous aqueous phase. Figure 1.4 gives a visual representation of an oil-in-water emulsion in which the droplets are stabilised by surface active emulsifiers, proteins, and solid particles. In oil-in-water emulsions, lipid oxidation is believed to primarily take place at the interface between the oil and water phases (McClements \& Decker, 2000; Waraho, McClements, \& Decker, 2011). Here, the unsaturated lipids are in contact with prooxidant compounds such as metal ions located in the aqueous layer (Cho, McClements, $\&$ Decker, 2002). Simple chemical reactions leading to lipid oxidation often cannot explain the transition from the initiation to propagation phase. In recent years, hypotheses on how the interplay of association colloids impact lipid oxidation mechanisms in emulsions has been developed (Budilarto \& Kamal-Eldin, 2015; Chaiyasit, Elias, McClements, \& Decker, 2007; Laguerre, Bily, Roller, \& Birtic, 2017). The emphasis of the proposed ideas and hypotheses primarily consider the importance of molecular transfer of reactants between and within emulsion droplets. The review of Laguerre et al. (2017) focuses on inter-droplet transfer and coins three ways in which compounds can transfer between emulsion droplets (Figure 1.4). The first possibility is that the compounds diffuse into the aqueous layer to reach neighbouring droplets. The second involves transfer of compounds between droplets upon droplet collision, while the third is about transfer of components using micelles as a vehicle. A fourth way is postulated by Budilarto and Kamal-Eldin (2015), where they consider inverse micelles in the oil droplets to play a critical role in the propagation steps. The commonality of these hypotheses is the critical role of hydroperoxides and secondary oxidation products and their amphiphilic properties. These hypotheses have been drafted to accommodate different type of emulsions, but when considering mayonnaise specifically, some are more likely than others. The first mechanism, regarding direct diffusion, was deemed unlikely since the amphiphilic or hydrophobic lipid oxidation products would freely migrate over the 
polar continuous water phase. For the second one, the transfer upon droplet collision is possible for such components, albeit slow due to the low dynamics in a mayonnaise. The third hypothesis proposed by Laguerre et al. (2017) is considered more likely, since if surfactants are present in excess, they could stimulate a fast inter-droplet transfer (Figure 1.4). These surfactants could form micelles in the continuous phase and transfer lipophilic components between emulsion droplets. This mechanism is thus far mainly hypothetical in the mayonnaise, but, in work by Raudsepp, Brüggemann, and Andersen (2014), it has been shown that the apolar peroxyl radicals can transfer between the oil droplets in oil-in-water emulsions, possibly via such micelle-assisted transfer. This observation shows that the lipid oxidation process is not restricted to the droplet in which it has been initiated, but can be spread between emulsion droplets. In contrast to the observations for peroxyl radicals, alkyl radicals were not found to take part in such a transfer (Raudsepp, Brüggemann, Knudsen, \& Andersen, 2016). The authors defined that the observed difference in the transfer is due to difference in radical life-times (Raudsepp et al., 2016), 10-6 $\mathrm{s}$ and 0.5-7 s for alkoxyl and peroxyl radicals, respectively (Pryor, 1986) that restricts the distance which a radical can travel before reacting. Laguerre et al. (2017) further elaborate on the topic by suggesting that lipid oxidation in food emulsion is unlikely to spread by radical transfer, due to the high reactivity that these species possess. They assume that LOOHs, as the first stable product of lipid oxidation are involved in transferring the reaction to new emulsion droplets. LOOHs are more polar than their precursors and are surface active compounds (Nuchi, Hernandez, McClements, \& Decker, 2002), both of which aid their diffusion into the aqueous phase. All this makes them a logical candidate to transfer the oxidation between emulsion droplets, forming micelles with and without the assistance of surfactants. In line with this hypothesis, the inverse might be happening (Figure 1.4). Reverse micelles might be able to capture prooxidant water soluble compounds from the interface and transport them throughout the lipid layer (Budilarto \& Kamal-Eldin, 2015; Chaiyasit et al., 2007). This process would also be dependent on a critical concentration of hydroperoxides $\left(\mathrm{CC}_{\mathrm{LOOH}}\right)$ formed at an oxidizable interface, after which hydroperoxides will stabilise reverse micelles and promote further oxidation inside the droplet. 


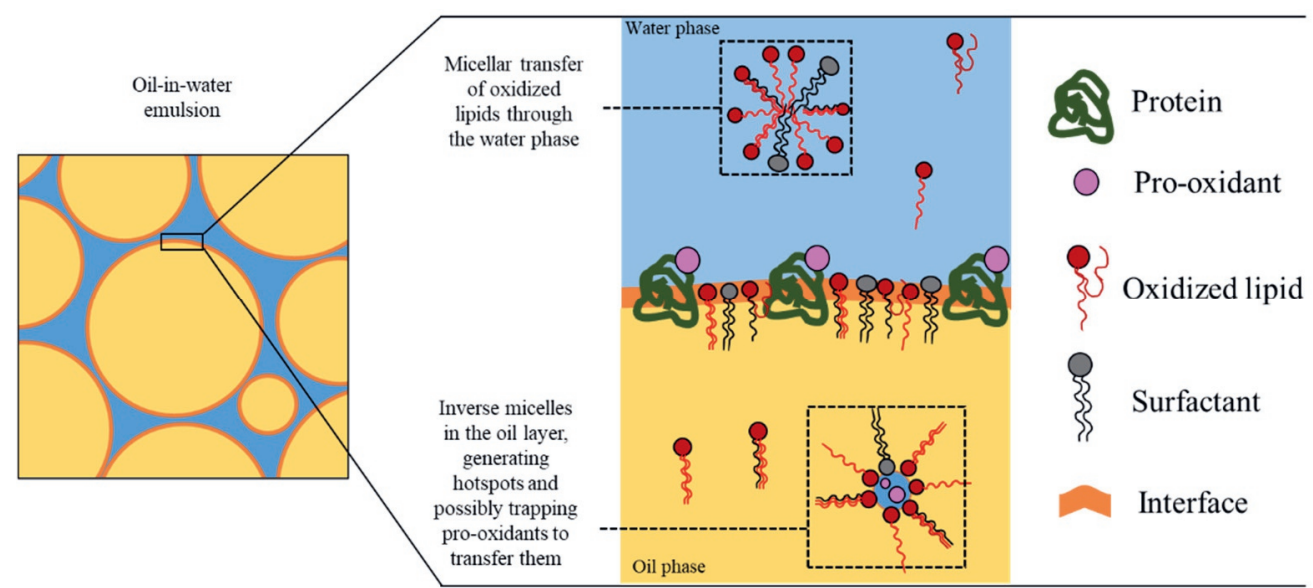

Figure 1.4. Hypothesised colloidal mechanisms for the increased oxidation in oil-in-water emulsions. In the water phase, micelles consisting of both oxidised lipids and surfactants might spread oxidation products throughout the emulsion. In the oil phase, inverse micelles might act as oxidation hotspots by trapping water-soluble pro-oxidants.

In all, the start of the propagation step will not only be dependent on the chemical susceptibility of the lipid compound to oxidation, but also the chemistry of the primary oxidation products formed. For instance, kinetics will be influenced by the ability of LOOH to form micelles with or without aid of surfactant molecules. Hypothetically, the transition happens when the Critical Micellar Concentration (CMC) of LOOH is reached and thus the reaction is no longer limited by the transfer of oxidative species via droplet collision. The transfer becomes micelle-assisted and thereby much faster. In other words, the reaction goes through a transition from transfer-limited to reaction-limited phase (Laguerre et al., 2017). For proper understanding of lipid oxidation in complex matrices, measuring and mapping oxidation products from all different routes is essential. Tools that are currently employed and/or available do not suffice for this purpose. Therefore, in this thesis, novel methods that fill this gap will be explored and utilised.

\subsection{Lipid oxidation assessment toolbox}

Accelerated shelf-life tests, or lipid oxidation samples in general are often monitored by simple one-dimensional methods. For the assessment of primary oxidation, the 'peroxide value (PV)' titration and the conjugated diene (CD) methods are often used. For aldehydes and epoxides, these are anisidine value (AV) and hydrogen bromide ( $\mathrm{HBr}$ ) titration methods, respectively. Alternatively, oxygen consumption in the headspace and/or product during shelf life can be measured, as a measure of overall oxidation. All these methods are indirect 
methods and have as major benefit that they are easy to perform in most labs, explaining their wide usage. However, besides being time-consuming and prone of analyst error, they are also aspecific, making it difficult to generate mechanistical insights in a reliable manner. This has driven an increased interest in rapid and multidimensional approaches for a more holistic description of lipid oxidation (E. N. Frankel \& Meyer, 2000).

\subsubsection{Chromatographic approaches}

The most well-known of these multidimensional methods are chromatography based. Here, both Gas Chromatography (GC) and Liquid Chromatography (LC) approaches have been used and developed, focusing on stable oxidation products. GC is primarily used to assess the volatiles, such as the aldehydes, ketones, alcohols and short carboxylic acids (Sghaier et al., 2016). The most common technique that is utilised here is Headspace (HS) GC, often coupled with a Mass Spectrometer (MS). In lipid oxidation studies, hexanal is measured most often as a marker for secondary oxidation by HS-GC as it is the major degradation product of linoleic acid hydroperoxides (E. N. Frankel, Hu, \& Tappel, 1989; Panseri, Soncin, Chiesa, $\&$ Biondi, 2011). For example, HS-GC-MS was used to establish that the oxidation rate of emulsified oils is larger than that of bulk oils (van Ruth \& Roozen, 2000). In another study, 2D-GC was applied in mayonnaise at low 'consumer relevant' temperature $\left(4-38^{\circ} \mathrm{C}\right)$, where the authors could also distinguish different pathways based on storage temperature (Ghorbani Gorji, Calingacion, Smyth, \& Fitzgerald, 2019). For oxidation products that are higher in molecular weight and are not volatile enough to be analysed with gas chromatography, LCmethods are often used, either coupled to a UV detector or MS. LC-MS has been successfully applied to detect and identify a range of primary and secondary oxidation products (Grüneis et al., 2019; Hollebrands, Varvaki, Kaal, \& Janssen, 2018; Steenhorst-Slikkerveer, Louter, Janssen, \& Bauer-Plank, 2000). LC-MS allows to look further than the oxidised moiety to the rest of the structure by looking at its mass. As such, hydroperoxides, aldehydes, epoxides can be assessed in combination with the fatty acid structure that is still present in the rest of the TAG. For example, one can distinguish between two-and-half TAGs that underwent the beta scission at different positions (e.g., 9 and 13 for LA). Current chromatographic methods are excellent for providing a framework on what types of lipid oxidation products are formed. However, the need for authentic standards and non-linear responses limits throughput when absolute quantification is desired. 


\subsubsection{Magnetic resonance}

Techniques that do not suffer from these drawbacks are spectroscopy methods such Nuclear Magnetic Resonance (NMR) and Electron Spin Resonance (ESR). To the wider audience, NMR is commonly linked with elucidation of chemical structure. For the last two decades, it is more and more applied for quantitative analysis as well. As the higher magnetic field strengths became more affordable and thus accessible, combined with significant developments in the processing software, such as deconvolution methods, NMR became a convenient option for quantitative analysis (Van Duynhoven, 2013). A major benefit and fundamental principle of quantitative NMR (qNMR) is that signal ratios directly correlate to molar ratios. This means that one can use generic internal/external standards for the quantification than authentic standards of every specific analyte. Since the electronic stability of NMR instruments, and that chromatographic separation is not required, qNMR methods are highly reproducible, both inter- and intra-laboratory. In recent years, qNMR methods have been applied widely in food research, from monosaccharide analysis after hydrolysis (Merkx et al., 2018) to profiling of tea metabolites (van der Hooft et al., 2012). Importantly, NMR is a very good tool for the assessment of lipid oxidation products as well. There has been a considerate amount of work published on the identification of aldehydes in oils (Skiera, Steliopoulos, Kuballa, Holzgrabe, \& Diehl, 2012b) under a wide variety of conditions. Furthermore, Xia et al. showed that NMR might be a good candidate for the epoxide assessment as well. Not only late oxidation products are detectable by NMR, but NMR also has potential for the quantification of hydroperoxides (Goicoechea \& Guillen, 2010; Skiera, Steliopoulos, Kuballa, Holzgrabe, \& Diehl, 2012a).

Whereas ${ }^{1} \mathrm{H}$ NMR can be used to assess and quantify stable oxidation products, Electron Spin Resonance (ESR), equally referred to as Electron Paramagnetic Resonance (EPR), allows the detection of radicals, which are the intermediates in lipid oxidation mechanisms. Indeed, with ESR, one can detect radical intermediates in lipid oxidation (Andersen \& Skibsted, 2002). Most radicals that are formed in food are highly reactive and therefore very short-lived intermediates (Andersen \& Skibsted, 2017). This results in low steady state concentrations that are below the detection limit of ESR. However, with the help of diamagnetic compounds (spin traps), the free radicals can be trapped resulting in more stable radicals, the so-called spin adducts. Usually, organic nitroso or nitrone compounds are used for this trapping purpose (Davies, 2016). Spin adducts can accumulate to ESR detectable concentrations and allow thereby the indirect detection of the free radicals formed during lipid oxidation. Spin traps do require covalent bonding with the target molecule, potentially influencing the mechanisms at play. 
Although the potential of using NMR and ESR to assess lipid oxidation is being slowly unlocked, to this day, these advanced analytical applications did not reach their full power. In this thesis, the focus is on unlocking the potential of these spectroscopic methods and to utilise them for building predictive quantitative models to better understand lipid oxidation in emulsions.

\subsection{Project aim}

Although widely used in industry, accelerated shelf-life tests are not used without drawbacks. First, it is well recognised that shelf-life testing under accelerating conditions (E. N. Frankel \& Meyer, 2000) may induce shifts in mechanisms, and therefore, different stability rankings than when performed under consumer-relevant conditions. Secondly, even under accelerated conditions, it can still take weeks of shelf-life before volatile secondary lipid oxidation products can be detected. Thirdly, it is difficult to infer the involved mechanism from these late lipid oxidation products. To optimise formulation and processing of oxidation-stable food emulsions in a more rapid and rational manner, there is a clear need for shelf-life tests that provide early (hours-days) markers that quantitatively predict generation of off-flavour and give an indication of the involved oxidative mechanism(s). The main goal of the thesis is to discover these mechanism-based early markers for lipid oxidation in food emulsions, possibly under consumer relevant conditions. The mechanistic underpinning of these markers should also allow the discrimination of the involved dominant pathways. To achieve this, we intend to develop rapid and quantitative NMR and ESR methods that cover major lipid oxidation initiators and intermediates.

\subsection{Outline of the thesis}

Chapter 2 describes the development of a quantitative NMR method that can be used in the quantitative assessment of both hydroperoxides and aldehydes. The common drawback of the lack of sensitivity in ${ }^{1} \mathrm{H}$ NMR is being tackled using band-selective excitation. Individual hydroperoxide and aldehyde signals will be assigned to allow for potential mechanistic markers. The optimised method will then be tested on a set of mayonnaises that are stored under different shelf-life conditions.

In Chapter 3, NMR is further explored to include the epoxides that were not quantifiable in Chapter 2. Here, 2D HSQC is used to quantify epoxides in a real food emulsion and oils under thermal oxidation conditions to enable assessment of environmental impact on lipid oxidation pathways. Furthermore, we will assess the potential of epoxides as an early marker. 
Different classes of epoxides are distinguished, based on stereospecificity and additional oxidation moieties.

In Chapter 4, the potential of ESR in combination with PBN spin-trapping as a technique for early assessment in lipid oxidation in mayonnaises will be explored. Furthermore, we will describe the spin-trapping mechanism of PBN in oil-systems and its implications on the interpretation of the kinetics.

In Chapter 5, we will use the early oxidation products to train a prediction model for late oxidation kinetics. To this, we will use an extensive set of kinetic curves acquired by the method described in Chapter 2. Different semi-empirical models will be tested with parameters that can be linked to the colloidal behaviour of the emulsions. The ultimate goal of Chapter 5 is to evaluate the potential of early mechanistical oxidation markers to predict the oxidative stability at a later stage to shorten the total length of shelf-life tests.

In Chapter 6, we will assess the colloidal role of phosvitin in early lipid oxidation kinetics. Specifically, the binding of phosvitin to iron in mayonnaise will be assessed by phosphorous NMR ( ${ }^{31} \mathrm{P}$ NMR). For this, the distinct magnetic properties of $\mathrm{Fe}(\mathrm{II})$ and $\mathrm{Fe}(\mathrm{III})$, which are respectively dia- and paramagnetic and thus have different line-broadening effects on the phosvitin signal, will be utilised. A holistic overview of how all chapters fit within the scope of this thesis is provided in Figure 1.5.

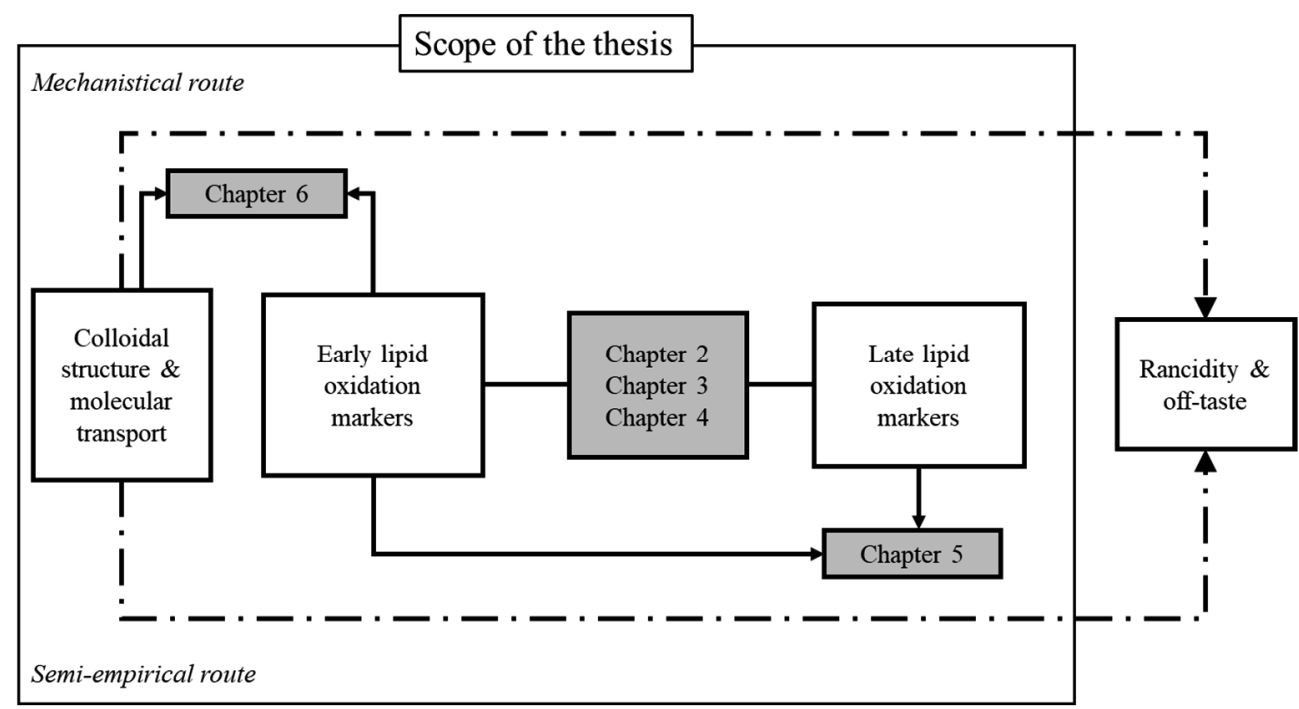

Figure 1.5. Schematic outline of the thesis, depicting the scope of this thesis. Chapters are positioned to provide a holistic overview as to which topic they had most impact on and which route (mechanistical/semi-empirical) they followed most closely. 


\subsection{Literature}

Alves, A. Q., da Silva, V. A. J., Góes, A. J. S., Silva, M. S., de Oliveira, G. G., Bastos, I. V. G. A., . . Alves, A. J. (2019). The Fatty Acid Composition of Vegetable Oils and Their Potential Use in Wound Care. Advances in Skin \& Wound Care, 32(8), 1-8.

Andersen, M. L., \& Skibsted, L. H. (2002). Detection of early events in lipid oxidation by electron spin resonance spectroscopy. European Journal of Lipid Science and Technology, 104(1), 65-68.

Berton, C., Ropers, M.-H., Bertrand, D., Viau, M., \& Genot, C. (2012). Oxidative stability of oil-in-water emulsions stabilised with protein or surfactant emulsifiers in various oxidation conditions. Food Chemistry, 131(4), 1360-1369.

Budilarto, E. S., \& Kamal-Eldin, A. (2015). The supramolecular chemistry of lipid oxidation and antioxidation in bulk oils. Eur J Lipid Sci Technol, 117(8), 1095-1137.

Calligaris, S., Manzocco, L., Anese, M., \& Nicoli, M. C. (2016). Shelf-life Assessment of Food Undergoing Oxidation-A Review. Critical Reviews in Food Science and Nutrition, 56(11), 1903-1912.

Chaiyasit, W., Elias, R. J., McClements, D. J., \& Decker, E. A. (2007). Role of physical structures in bulk oils on lipid oxidation. Critical Reviews in Food Science and Nutrition, 47(3), 299-317.

Cho, Y.-J., McClements, D. J., \& Decker, E. A. (2002). Ability of Surfactant Micelles To Alter the Physical Location and Reactivity of Iron in Oil-in-Water Emulsion. Journal of Agricultural and Food Chemistry, 50(20), 5704-5710.

Davies, M. J. (2016). Detection and characterisation of radicals using electron paramagnetic resonance (EPR) spin trapping and related methods. Methods, 109, 21-30.

Frankel, E. N. (2014). Lipid Oxidation: Elsevier Science.

Frankel, E. N., Hu, M.-L., \& Tappel, A. L. (1989). Rapid headspace gas chromatography of hexanal as a measure of lipid peroxidation in biological samples. Lipids, 24(11), 976.

Frankel, E. N., \& Meyer, A. S. (2000). The problems of using one-dimensional methods to evaluate multifunctional food and biological antioxidants. Journal of the Science of Food and Agriculture, 80(13), 1925-1941.

Ghorbani Gorji, S., Calingacion, M., Smyth, H. E., \& Fitzgerald, M. (2019). Comprehensive profiling of lipid oxidation volatile compounds during storage of mayonnaise. Journal of food science and technology, 56(9), 4076-4090.

Goicoechea, E., \& Guillen, M. D. (2010). Analysis of Hydroperoxides, Aldehydes and Epoxides by $1 \mathrm{H}$ Nuclear Magnetic Resonance in Sunflower Oil Oxidized at 70 and $100{ }^{\circ} \mathrm{C}$. Journal of Agricultural and Food Chemistry, 58(10), 6234-6245.

Gómez-Alonso, S., Mancebo-Campos, V., Desamparados Salvador, M., \& Fregapane, G. (2004). Oxidation kinetics in olive oil triacylglycerols under accelerated shelf-life testing $\left(25-75^{\circ} \mathrm{C}\right)$. European Journal of Lipid Science and Technology, 106(6), 369-375.

Grüneis, V., Fruehwirth, S., Zehl, M., Ortner, J., Schamann, A., König, J., \& Pignitter, M. (2019). Simultaneous Analysis of Epoxidized and Hydroperoxidized Triacylglycerols in Canola Oil and Margarine by LC-MS. Journal of Agricultural and Food Chemistry, 67(36), 10174-10184. 
Gutteridge, J. M. C., Richmond, R., \& Halliwell, B. (1979). Inhibition of the iron-catalysed formation of hydroxyl radicals from superoxide and of lipid peroxidation by desferrioxamine. Biochemical Journal, 184(2), 469-472.

Hollebrands, B., Varvaki, E., Kaal, S., \& Janssen, H.-G. (2018). Selective labeling for the identification and semi-quantification of lipid aldehydes in food products. Analytical and Bioanalytical Chemistry, 410(22), 5421-5429.

Holman, R. T., \& Elmer, O. C. (1947). The rates of oxidation of unsaturated fatty acids and esters. Journal of the American Oil Chemists Society, 24(4), 127-129.

Jacobsen, C., Hartvigsen, K., Thomsen, M. K., Hansen, L. F., Lund, P., Skibsted, L. H., . . . Meyer, A. S. (2001). Lipid Oxidation in Fish Oil Enriched Mayonnaise: Calcium Disodium Ethylenediaminetetraacetate, but Not Gallic Acid, Strongly Inhibited Oxidative Deterioration. Journal of Agricultural and Food Chemistry, 49(2), 10091019.

Jacobsen, C., Let, M. B., Nielsen, N. S., \& Meyer, A. S. (2008). Antioxidant strategies for preventing oxidative flavour deterioration of foods enriched with n-3 polyunsaturated lipids: a comparative evaluation. Trends in Food Science \& Technology, 19(2), 76-93.

Johnson, D. R., \& Decker, E. A. (2015). The Role of Oxygen in Lipid Oxidation Reactions: A Review. Annual Review of Food Science and Technology, 6(1), 171-190.

Laguerre, M., Bily, A., Roller, M., \& Birtic, S. (2017). Mass Transport Phenomena in Lipid Oxidation and Antioxidation. Annual Review of Food Science and Technology, 8(1), 391-411.

Manzocco, L., Calligaris, S., Anese, M., \& Nicoli, M. C. (2016). Chapter 2 - Determination and Prediction of Shelf Life of Oils/Fats and Oil/Fat-Based Foods. In M. Hu \& C. Jacobsen (Eds.), Oxidative Stability and Shelf Life of Foods Containing Oils and Fats (pp. 133-156): AOCS Press.

McClements, D., \& Decker, E. A. (2000). Lipid Oxidation in Oil-in-Water Emulsions: Impact of Molecular Environment on Chemical Reactions in Heterogeneous Food Systems. Journal of Food Science, 65(8), 1270-1282.

Merkx, D. W. H., Westphal, Y., van Velzen, E. J. J., Thakoer, K. V., de Roo, N., \& van Duynhoven, J. P. M. (2018). Quantification of food polysaccharide mixtures by $1 \mathrm{H}$ NMR. Carbohydrate Polymers, 179, 379-385.

Nuchi, C. D., Hernandez, P., McClements, D. J., \& Decker, E. A. (2002). Ability of Lipid Hydroperoxides To Partition into Surfactant Micelles and Alter Lipid Oxidation Rates in Emulsions. Journal of Agricultural and Food Chemistry, 50(19), 54455449.

Panseri, S., Soncin, S., Chiesa, L. M., \& Biondi, P. A. (2011). A headspace solid-phase microextraction gas-chromatographic mass-spectrometric method (HS-SPMEGC/MS) to quantify hexanal in butter during storage as marker of lipid oxidation. Food Chem, 127(2), 886-889.

Pryor, W. A. (1986). Oxy-Radicals and Related Species: Their Formation, Lifetimes, and Reactions. Annual Review of Physiology, 48(1), 657-667.

Ragnarsson, J. O., \& Labuza, T. P. (1977). Accelerated shelf-life testing for oxidative rancidity in foods-A review. Food Chemistry, 2(4), 291-308.

Raudsepp, P., Brüggemann, D. A., \& Andersen, M. L. (2014). Evidence for Transfer of Radicals between Oil-in-Water Emulsion Droplets as Detected by the Probe (E,E)3,5-Bis(4-phenyl-1,3-butadienyl)-4,4-difluoro-4-bora-3a,4a-diaza-s-indacene, 
BODIPY665/676. Journal of Agricultural and Food Chemistry, 62(51), 1242812435.

Raudsepp, P., Brüggemann, D. A., Knudsen, J. C., \& Andersen, M. L. (2016). Localized lipid autoxidation initiated by two-photon irradiation within single oil droplets in oil-inwater emulsions. Food chemistry, 199, 760-767.

Schaich, K. M. (2012). Thinking outside the classical chain reaction box of lipid oxidation. Lipid Technology, 24(3), 55-58.

Schröder, A., Sprakel, J., Boerkamp, W., Schroën, K., \& Berton-Carabin, C. C. (2019). Can we prevent lipid oxidation in emulsions by using fat-based Pickering particles? Food Research International, 120, 352-363.

Sghaier, L., Vial, J., Sassiat, P., Thiebaut, D., Watiez, M., Breton, S., . . Cordella, C. B. Y. (2016). An overview of recent developments in volatile compounds analysis from edible oils: Technique-oriented perspectives. European Journal of Lipid Science and Technology, 118(12), 1853-1879.

Skiera, C., Steliopoulos, P., Kuballa, T., Holzgrabe, U., \& Diehl, B. (2012a). 1H-NMR Spectroscopy as a New Tool in the Assessment of the Oxidative State in Edible Oils. Journal of the American Oil Chemists' Society, 89, 1383-1391.

Skiera, C., Steliopoulos, P., Kuballa, T., Holzgrabe, U., \& Diehl, B. (2012b). 1H NMR approach as an alternative to the classical p-anisidine value method. European Food Research and Technology, 235, 1101-1105.

Steenhorst-Slikkerveer, L., Louter, A., Janssen, H.-G., \& Bauer-Plank, C. (2000). Analysis of nonvolatile lipid oxidation products in vegetable oils by normal-phase highperformance liquid chromatography with mass spectrometric detection. Journal of the American Oil Chemists' Society, 77(8), 837.

van der Hooft, J. J. J., de Vos, R. C. H., Mihaleva, V., Bino, R. J., Ridder, L., de Roo, N., . . - Vervoort, J. (2012). Structural Elucidation and Quantification of Phenolic Conjugates Present in Human Urine after Tea Intake. Analytical Chemistry, 84(16), 7263-7271.

Van Duynhoven, J., van Velzen, E. \& Jacobs, D. M. . (2013). Quantification of complex mixtures by NMR. Annual Reports on NMR Spectroscopy, 80.

van Ruth, S. M., \& Roozen, J. P. (2000). Aroma compounds of oxidised sunflower oil and its oil-in-water emulsion: volatility and release under mouth conditions. European Food Research and Technology, 210(4), 258-262.

Waraho, T., McClements, D. J., \& Decker, E. A. (2011). Mechanisms of lipid oxidation in food dispersions. Trends in Food Science \& Technology, 22(1), 3-13. 


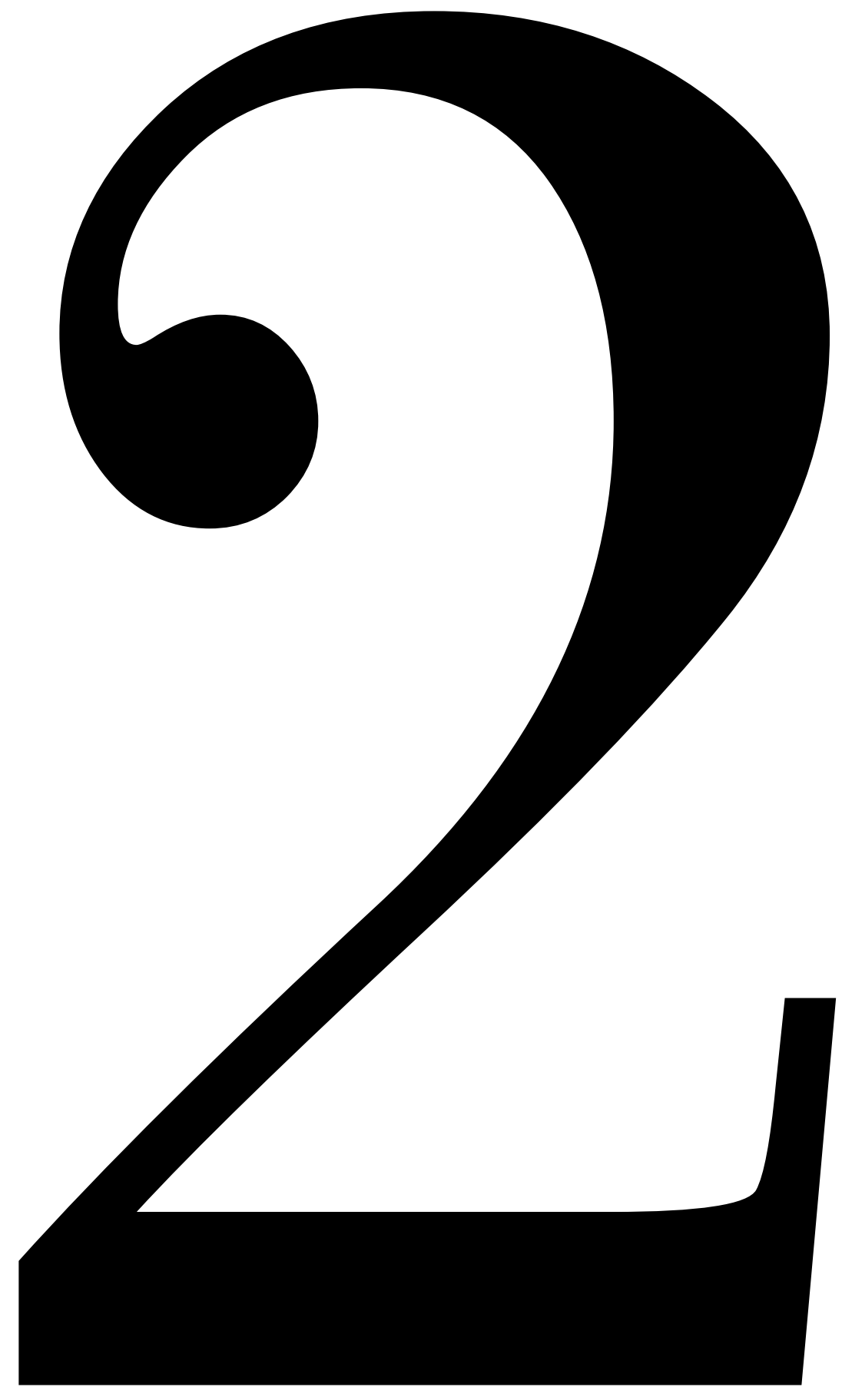




\section{Chapter 2}

\section{Rapid quantitative profiling of lipid oxidation products in a food emulsion by ${ }^{1} \mathrm{H}$ NMR}

Based on: Merkx, D. W. H., Hong, G. T. S., Ermacora, A., \& van Duynhoven, J. P. M. (2018). Rapid Quantitative Profiling of Lipid Oxidation Products in a Food Emulsion by ${ }^{1} \mathrm{H}$ NMR. Analytical Chemistry, 90(7), 4863-4870. 


\begin{abstract}
Lipid oxidation is one of the most important reasons for the compromised shelf life of food emulsions. A major bottleneck in unravelling the underlying mechanisms is the lack of methods that provide a rapid, quantitative and comprehensive molecular view on lipid oxidation in these heterogeneous systems. In this study, the unbiased and quantitative nature of ${ }^{1} \mathrm{H}$ NMR was exploited to assess lipid oxidation products in mayonnaise, a particularly oxidation-prone food emulsion. An efficient and robust procedure was implemented to produce samples where the ${ }^{1} \mathrm{H}$ NMR signals of oxidation products could be observed in a well resolved and reproducible manner. ${ }^{1} \mathrm{H}$ NMR signals of hydroperoxides were assigned in a fatty acid and isomer specific way. Band-selective ${ }^{1} \mathrm{H}$ NMR pulse excitation allowed immediate quantification of both hydroperoxides and aldehydes with high throughput and high dynamic range at levels of $0.03 \mathrm{mmol} / \mathrm{kg}$ with high precision $\left(\mathrm{RSD}_{\mathrm{R}}=5.9 \%\right)$. Explorative multivariate data modelling of the quantitative ${ }^{1} \mathrm{H}$ NMR profiles revealed that shelf-life temperature has a significant impact on lipid oxidation mechanisms.
\end{abstract}




\subsection{Introduction}

Unsaturated fatty acids are essential to the human diet and are for a major part consumed in emulsified form. In food emulsions such as mayonnaise and dressings, unsaturated fatty acids are prone to lipid oxidation, a chemical process that consists of two phases. In the primary oxidation phase, hydroperoxides are formed via radical mechanisms. These hydroperoxides then further degrade to form secondary oxidation products, such as aldehydes, hydroxides and epoxides (Coupland \& McClements, 1996; Jacobsen, 1999; McClements \& Decker, 2000). Many of these secondary oxidation products are volatile and perceived by the consumer as rancid, which compromises the sensorial quality of food emulsions.

Compared to bulk oil, lipid oxidation mechanisms in oil-in-water food emulsions become more complex due to the presence of oil-water interfaces (McClements \& Decker, 2000). In these emulsions, the presence of transition metals and antioxidants at the droplet interface can respectively enhance and retard lipid oxidation (Mozuraityte, Rustad, \& Storrø, 2008). Additionally, fatty acids become more hydrophilic after primary oxidation, making them more exposed at the interface and thereby more vulnerable for further oxidation reactions (Berton-Carabin, Ropers, \& Genot, 2014; Ghorbani Gorji, Smyth, Sharma, \& Fitzgerald, 2016). Furthermore, colloidal transport of reaction intermediates can modulate oxidation mechanisms (Budilarto \& Kamal-Eldin, 2015; Laguerre et al., 2015; Laguerre, Bily, Roller, $\&$ Birtic, 2017). The interplay of lipid oxidation chemistry with these colloidal effects and environmental conditions, such as temperature and oxygen pressure, is poorly understood. To better understand the mechanisms at play, a comprehensive view on lipid oxidation is required. One-dimensional methods (Frankel \& Meyer, 2000). such as Peroxide Value (PV) ("ISO 3960:2017," 2017) and Anisidine Value (AV) ("ISO 6885:2016," 2016), are unable to unravel different oxidation mechanisms. Therefore, we deem it necessary to adopt a 'foodomics' approach (Laghi, Picone, \& Capozzi, 2014), where the broad coverage of lipid oxidation products is combined with explorative multivariate modelling. Currently, no methods provide the required rapid, quantitative, and comprehensive molecular view on both primary and secondary lipid oxidation. LC-MS (Laguerre et al., 2015) can detect and identify a wide range of primary and secondary oxidation products in a single run, but suffers from low throughput and the need of authentic standards for quantification. Volatile secondary lipid oxidation products can sensitively be monitored by headspace GC (Frankel, Hu, \& Tappel, 1989), but quantification is compromised by partitioning over sample and headspace and the need for authentic standards.

Quantitative ${ }^{1} \mathrm{H}$ NMR (qNMR) profiling has been introduced as a high-throughput method that does not suffer from these drawbacks. NMR has been shown to identify (Guillen \& Goicoechea, 2009; Ibargoitia, Sopelana, \& Guillen, 2014) and quantify (Skiera, Steliopoulos, 
Kuballa, Holzgrabe, \& Diehl, 2012a, 2012b) primary and secondary lipid oxidation products in bulk oils by using a binary $\mathrm{CDCl} 3$ :DMSO-d6 solvent. These investigations focused on bulk oils in advanced stages of oxidation, and did not address the structural complexity of oil-in-water emulsions. In these systems, the presence of susceptibility mismatches at the water-oil interfaces leads to significant line broadening and thus compromises chemical shift resolution. Furthermore, levels of non-oxidised lipids and hydroperoxides differed four-tofive orders in magnitude, which poses a challenge to the dynamic range of the receiver. In this paper, we describe the development and optimization of an ${ }^{1} \mathrm{H}$ NMR based profiling method that covers a wide range of primary and major secondary oxidation products in food emulsions. The method makes use of band selective pulses (Dugo et al., 2015; Rastrelli, 2009), is quantitative, sensitive, unbiased and allows for high sample throughput. The suitability of the ${ }^{1} \mathrm{H}$ NMR profiling approach for distinguishing temperature induced shifts on the lipid oxidation mechanism was demonstrated in a mayonnaise shelf-life test.

\subsection{Material and Methods}

\subsubsection{Materials}

$\mathrm{CDCl}_{3}$, DMSO-d $\mathrm{d}_{6}$ and $4 \AA$ molsieves were purchased from Euriso-top (Saint-Aubin, France). Methyl oleate, methyl linoleate, methyl $\alpha$-linolenate, glyceryl trioleate, glyceryl trilinoleate and glyceryl trilinolenate were all purchased from Sigma Aldrich (Zwijndrecht, the Netherlands). Medium chained tryglyceride (MCT) oil was prepared in-house by stripping coconut oil.

\subsubsection{Mayonnaise formulation and processing}

Mayonnaise was manufactured by emulsification in a colloid mill. The aqueous phase was prepared first, with the following ingredients: egg yolk $(4.2 \% \mathrm{w} / \mathrm{w})$, starch $(1 \% \mathrm{w} / \mathrm{w})$, sorbic acid $(0.01 \% \mathrm{w} / \mathrm{w})$, sodium chloride $(1.1 \% \mathrm{w} / \mathrm{w})$, sugar $(1.3 \% \mathrm{w} / \mathrm{w})$, water $(20.1 \% \mathrm{w} / \mathrm{w})$. Subsequently, rapeseed oil $(65 \% \mathrm{w} / \mathrm{w})$ and spirit vinegar $12 \%(2.6 \% \mathrm{w} / \mathrm{w})$ were slowly added to form the emulsion. 


\subsubsection{Mayonnaise storage}

Aliquots of $1 \mathrm{~g}$ mayonnaise were stored in $20 \mathrm{~mL}$ screwcap vials in the dark at $20^{\circ} \mathrm{C}$ and 50 ${ }^{\circ} \mathrm{C}$. At $20^{\circ} \mathrm{C}$, five aliquots were removed from storage each week for 29 weeks. At $50{ }^{\circ} \mathrm{C}$, five aliquots were removed from storage two to three times a week for 6 weeks. The aliquots were then stored at $-20^{\circ} \mathrm{C}$ until further analysis.

\subsubsection{Nuclear Magnetic Resonance (NMR) sample preparation}

For NMR, two aliquots were used per formulation and timepoint. These were stored at -20 ${ }^{\circ} \mathrm{C}$ for $16 \mathrm{~h}$ and subsequently thawed at room temperature, resulting in phase separation of the oil and water phases. $250 \mu \mathrm{L}$ of the upper layer (oil) was collected and transferred to a $2 \mathrm{~mL}$ Eppendorf tube. To this, $750 \mu \mathrm{L}$ of 5:1 $\mathrm{CDCl}_{3}: \mathrm{DMSO}-\mathrm{d}_{6}$ (Euriso-top, France) was added. This solvent was dried using $4 \AA$ molsieves (Euriso-top, France). After homogenizing the solution, $550 \mu \mathrm{L}$ was transferred to a 5-mm NMR tube.

\subsubsection{NMR acquisition}

Spectra were recorded on a Bruker Avance HD $700 \mathrm{MHz}$ NMR spectrometer (Bruker BioSpin, Switzerland) equipped with a 5-mm BBI-probe or an Avance III $600 \mathrm{MHz}$ spectrometer equipped with a 5-mm cryo-probe. The internal temperature of the probe was set at $295 \mathrm{~K}$. For each sample, a single pulse and a band-selective experiment were performed. The single pulse experiments were recorded with 4 scans, a relaxation time of 5 sec and an acquisition time of 4 seconds. The $90^{\circ}$ pulse length was determined automatically $(\sim 7.2 \mu \mathrm{s})$, the receiver gain was set to the maximum value where no digitiser overflow occurred. Two band selective experiments were used. The first one used a hard $90^{\circ}$ pulse followed by a selective gradient enhanced Gaussian inversion pulse (Berger, 1997). The second one used a double echo with gradients using selective refocussing with a RE-BURP shaped pulse and a $90^{\circ}$ pulse in between the $180^{\circ}$ pulses to refocus J-evolution (Adams, Holroyd, Aguilar, Nilsson, \& Morris, 2013; Melliou, Magiatis, \& Killday, 2015). The length of the shaped pulses was 1-2 ms, and band-selective spectra were recorded with 16-32 scans. The relaxation and acquisition times were respectively set to 5 and $2.7 \mathrm{~s}$, the $90^{\circ}$ pulse length was determined automatically $(\sim 7.2 \mu \mathrm{s})$. The data was processed with Bruker TopSpin 3.2 software. Before Fourier transformation, an exponential window function with a line broadening factor of 0.3 was applied, followed by automatic baseline correction and phase correction. Band-selective NOESY and TOCSY experiments were used for spectral assignments (Berger, 1997). 


\subsubsection{Calculations}

The levels of peroxides and aldehydes $\left(\mathrm{c}_{\mathrm{ox}}\right)$ are both expressed in $\mathrm{mmol} / \mathrm{kg}$ and are calculated by Equation 1 for a single pulse experiment recorded under quantitative (van Duynhoven, van Velzen, \& Jacobs, 2013) acquisition conditions:

$$
\mathrm{c}_{\mathrm{ox}}(\mathrm{mmol} / \mathrm{kg})=\frac{\mathrm{I}_{\mathrm{ox}}}{\mathrm{I}_{\mathrm{TG}}} \cdot \frac{\mathrm{N}_{\mathrm{TG}}}{\mathrm{N}_{\mathrm{ox}}} \cdot \frac{10^{6}}{\mathrm{MW}_{\mathrm{TG}}}
$$

and by Equation 2 for spectra recorded with band selective excitation pulses:

$$
\begin{aligned}
\mathrm{c}_{\mathrm{ox}}(\mathrm{mmol} / \mathrm{kg})= & \frac{\mathrm{I}_{\mathrm{ox}}}{\mathrm{I}_{\mathrm{TG}}} \cdot \frac{\mathrm{N}_{\mathrm{TG}}}{\mathrm{N}_{\mathrm{ox}}} \cdot \frac{\mathrm{ns}_{\mathrm{zg}}}{\mathrm{ns}_{\mathrm{sel}}} \cdot \frac{\sin \left(\mathrm{p}_{\mathrm{zg}}\right)}{\sin \left(\mathrm{p}_{\mathrm{sel}}\right)} \cdot \frac{\mathrm{RG}_{\mathrm{zg}}}{\mathrm{RG}_{\mathrm{sel}}} \cdot \frac{10^{6}}{\mathrm{MW}_{\mathrm{TG}}} \quad \text { Eq. } 2.2 \\
& \cdot \text { SelFactor }
\end{aligned}
$$

Here, $\mathbf{I}$ is the NMR signal intensity after signal integration, $\mathrm{N}$ is the number of protons, $\mathrm{MW}$ is the molecular weight (average triglyceride $\sim 880 \mathrm{~g} \cdot \mathrm{mol}^{-1}$ ), ns is the number of scans, RG is the receiver gain, $\mathrm{p}$ is the pulse angle in degrees. Index ox stands for oxidized lipid product, TG for triglyceride, zg for the direct pulse experiment and sel for the band-selective experiment. SelFactor stands for the empirically determined factor which corrects for the signal intensity loss due to relaxation differences between the single pulse and band-selective experiments. The spectral integration region to determine $\mathrm{I}_{\mathrm{TG}}$ is $\delta 4.4-4.0 \mathrm{ppm}$, with $\mathrm{N}_{\mathrm{TG}}$ corresponding to the 4 outer protons of the glycerol backbone. The glycerol backbone is not expected to undergo changes upon oxidation, hence its ${ }^{1} \mathrm{H}$ NMR signal can be used as internal standard (Skiera et al., 2012a). The integrals $\mathrm{I}_{\mathrm{ox}}$ are determined from the spectral integration regions listed in Table 2.1, all with $\mathrm{N}_{\mathrm{ox}}$ corresponding to 1 .

\subsubsection{Peroxide Value (PV)}

PV values were determined according to the international standard procedure ISO 3960 ("ISO 3960:2017," 2017). The method in short: $5.0 \mathrm{~g}$ oil was added to a $25 \mathrm{~mL}$ isooctane/acetic acid solution and a $1.0 \mathrm{~mL}$ saturated potassium iodide solution. After 5 minutes, the reaction was quenched with $100 \mathrm{~mL}$ water and $2.0 \mathrm{~mL}$ starch solution was added. The mixture was titrated with a sodium thiosulfate solution until the dark blue solution became transparent.

\subsubsection{Headspace Gas Chromatography (HS-GC)}

For method comparison, mayonnaise samples were measured with both Headspace GC as well as ${ }^{1} \mathrm{H}$ NMR. To semi-quantitatively assess the hexanal content, a hexanal in MCT-oil 
standard sample was included in the measurement series. The headspace GC analyses were performed on a 7890A gas chromatograph (Agilent Technologies, United States). The screwcap vials were placed in an automated headspace sampler at $60{ }^{\circ} \mathrm{C}$ and allowed to equilibrate for at least 30 minutes. Headspace samples of $0.5 \mathrm{~mL}$ were injected with a gastight syringe in split mode (ratio 1:40) onto a DB Wax column (J\&W Scientific, Belgium) with specifications: length $20 \mathrm{~m}$, ID $0.18 \mathrm{~mm}$ and film thickness of $0.3 \mu \mathrm{m}$. The GC was equipped with a 5975C MS detector (Agilent Technologies, United States) using an electron source of $70 \mathrm{eV}, \mathrm{m} / \mathrm{z} 33-150$ and solvent delay of 0.75 minutes. Temperatures at the inlet and detector were respectively $200{ }^{\circ} \mathrm{C}$ and $230{ }^{\circ} \mathrm{C}$. The temperature program for GC was: $35^{\circ} \mathrm{C}$ $(2 \mathrm{~min})-35{ }^{\circ} \mathrm{C} / \mathrm{min}$ to $200{ }^{\circ} \mathrm{C}-200{ }^{\circ} \mathrm{C}$ (2 min). The helium gas flow through the column was $1.0 \mathrm{~mL} / \mathrm{min}$. Peak areas (target response) of hexanal were monitored and compared with the standard sample (hexanal in MCT oil) to semi-quantify the hexanal content.

\subsubsection{Validation}

Repeatability (r) and within-laboratory reproducibility $(\mathrm{R})$ were determined by measuring three samples in duplicate for four days (24 measurements in total).(Miller \& Miller, 2010) The samples were stored in the freezer $\left(-20^{\circ} \mathrm{C}\right)$ prior to analysis. For hydroperoxides and aldehydes, respectively three and two different concentration levels were analyzed. The $r$ and $\mathrm{R}$ for each concentration level were obtained using an ANalysis Of VAriance (ANOVA) model. Results were expressed as relative standard deviations of repeatability $\left(\mathrm{RSD}_{\mathrm{r}}\right)$ and within-laboratory reproducibility $\left(\mathrm{RSD}_{\mathrm{R}}\right)$. The limit of detection $(\mathrm{LoD})$ and limit of quantification (LoQ) were respectively based on estimates of ten and thirty times the standard deviation from ten samples containing small amounts of oxidation products. The trueness of results obtained by application of the was determined by measuring a set of 5 oil sample in duplicate with both the ${ }^{1} \mathrm{H}$ NMR method and PV titration method. Method offset and proportional bias were determined by linear regression.

\subsubsection{Principal Component Analysis (PCA)}

Principal component analysis (PCA) was performed using SIMCA 14 (Umetrics, Sweden). Twenty quantified concentrations of the oxidation products were used as PCA input. Oxidation products below the detection limit were set at 0 . The first three principal components were calculated. Pareto scaling without variable centering (ParN) was applied to the data set. 


\subsection{Results and Discussion}

\subsubsection{NMR sample preparation}

For the analysis of lipid oxidation products in food emulsions we used a binary solvent mixture, composed of 5:1 CDCl 3 :DMSO-d $\mathrm{d}_{6}$ (Skiera et al., 2012a). The binary solvent was used because DMSO forms hydrogen bonds with hydroperoxides, which in turn narrowed the width of hydroperoxide signals and moved them downfield, resulting in well dispersed peaks. However, when dissolving a full emulsion into this binary solvent, the present water partially dissolved as well. This water then broadened the hydroperoxide peaks significantly and effectively countered the effect DMSO had on line-width and dispersion. To avoid this, we extracted the lipids prior to analysis. A quick and convenient method to achieve this was by breaking the emulsion in a freeze-thaw step. This resulted into a clear separation of the aqueous (bottom) and oil (top) phases, after which the latter could conveniently be transferred for further analysis. The freeze-thaw step complied well with the logistic requirements of shelf-life tests where samples are typically stored in a freezer at either $-20{ }^{\circ} \mathrm{C}$ or $-80{ }^{\circ} \mathrm{C}$ between sampling and actual measurement.

To investigate whether the oil-layer correctly represented the oil in the food emulsion, we compared the freeze-thaw extraction with the established, but labour-intensive, chloroformmethanol extraction (Folch, Lees, \& Sloane Stanley, 1957). We found no significant differences in hydroperoxide concentrations between the two extraction methods (see Supporting Info, Figure S2.1). Compared to the samples obtained by chloroform extraction, the linewidths of the hydroperoxide signals in samples produced by freeze-thawing were narrower. This can be attributed to the presence of water in the chloroform extracts, which compromises the line narrowing effect of the binary solvent.

\subsubsection{Dynamic range improvement}

In the early stages of lipid oxidation, primary oxidation products are typically present at ppm level. Therefore, in $\mathrm{CDCl}_{3}$ :DMSO- $\mathrm{d}_{6}$ lipid extracts, signals of these oxidation products could be up to five orders of magnitude smaller than the abundant background signals of nonoxidised lipids. Even with a modern receiver, digitiser noise compromised the detection of such low levels (Figure 2.1A). To reduce the digital noise in the hydroperoxide and aldehyde regions, the signal of the abundant non-oxidised lipids needed to be suppressed. For this purpose, we excited the signals of the hydroperoxides and aldehydes with a 1D band selective gradient pulse using a shaped Gaussian inversion pulse (Berger, 1997). This pulse sequence required separate acquisitions where respectively the hydroperoxide region $(\delta$ 11.5-10.5 
ppm) and the aldehyde region ( $\delta$ 10.0-9.0 ppm) were excited. By using a RE-BURP shaped pulse (Adams et al., 2013), however, we were able to uniformly excite both the hydroperoxide $(\delta 11.5-10.5 \mathrm{ppm})$ and aldehyde signals $(\delta 10.0-9.0 \mathrm{ppm})$. This pulse program effectively reduced the overall measurement time by a factor two, while retaining signal-tonoise ratio.

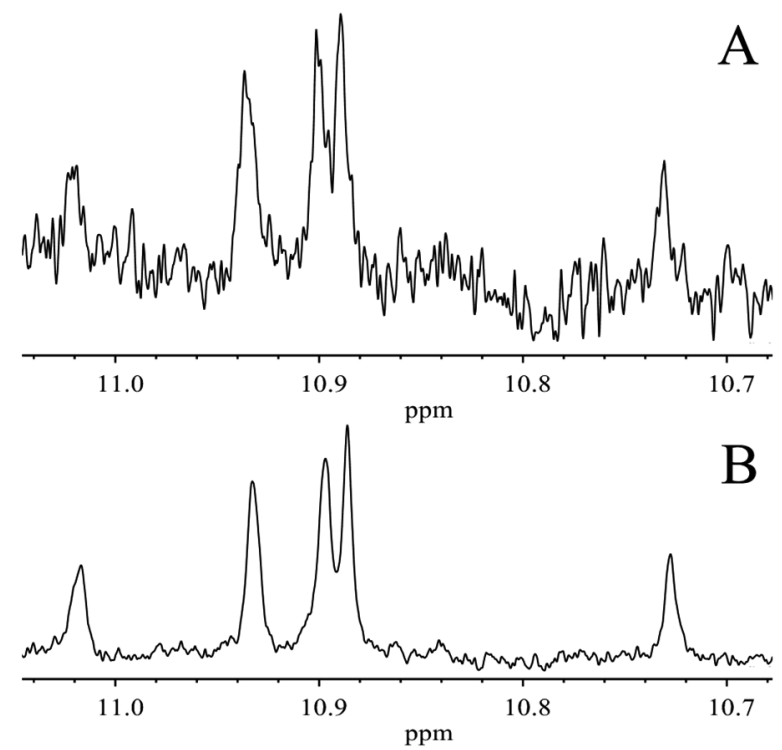

Figure 2.1. The ${ }^{1} \mathrm{H}$ NMR spectrum $(700 \mathrm{MHz})$ of the hydroperoxide region of a $\mathrm{CDCl}_{3}: \mathrm{DMSO} \mathrm{d}_{6}$ lipid extract prepared from mildly oxidised mayonnaise sample. The top spectrum (A) is recorded with a regular excitation with a hard $90^{\circ}$ pulse, the bottom spectrum (B) with a band-selective pulse.

The use of band-selective pulses allowed for a significant increase of receiver gain, resulting in improved digitization and a threefold improved signal-to-noise ratio (Figure 2.1B). The use of a ${ }^{1} \mathrm{H}$ NMR frequency of $700 \mathrm{MHz}$ relative to $600 \mathrm{MHz}$ did not yield better resolved hydroperoxides and aldehydes signals. This can be attributed to the linewidths of these signals which are typically 1-2 Hz. Hence, we proceeded by recording quantitative NMR profiles using a $600 \mathrm{MHz}$ NMR instrument equipped with a 5-mm cryoprobe.

\subsection{3. ${ }^{1} \mathrm{H}$ NMR spectral assignments of hydroperoxide and aldehyde signals}

Detailed assignments of ${ }^{1} \mathrm{H}$ NMR aldehyde signals in the binary $\mathrm{CDCl}_{3}: \mathrm{DMSO}-\mathrm{d}_{6}$ solvent were derived from literature (Guillen \& Goicoechea, 2009; Guillén \& Ruiz, 2008; Skiera et al., 2012b). The chemical shifts of these signals (Table 2.2) were stable within large sample series, rendering them useful for assessment in profiling mode. To date, hydroperoxide ${ }^{1} \mathrm{H}$ NMR signals in the binary $\mathrm{CDCl}_{3}: \mathrm{DMSO}-\mathrm{d}_{6}$ solvent have been assigned for three commonly occurring unsaturated fatty acids: oleic acid (O, 18:1), linoleic acid (LA, 18:2) and $\alpha$-linolenic 
acid ( $\alpha$ Ln, 18:3) (Skiera et al., 2012a). These ${ }^{1} \mathrm{H}$ NMR assignments were for a major part derived from the oxidation products of the methyl esters of these fatty acids. To obtain more specific assignments, we pursued a more a detailed structural elucidation of these products. However, established 2D homo- and heteronuclear NMR techniques could not be used due to the unfavorable dynamic range of the levels of oxidation products vs non-oxidised lipids. Hence, 1D band-selective NOESY and TOCSY pulse sequences (Berger, 1997) were deployed to obtain assignments specific for hydroperoxide position in the fatty acid chain and cis-trans isomerism. 1D NOESY experiments were used to transfer the magnetization from the hydroperoxide $(\mathrm{OOH})$ to the neighboring allylic proton $(\mathbf{C H})$. After identifying the allylic proton, we used 1D TOCSY experiments to further transfer magnetization to neighboring olefinic protons $(\mathbf{H C}=\mathrm{CH})$. The J-coupling of these olefinic protons indicated whether the originally excited hydroperoxide is adjacent to a cis- or trans-configured double bond. This allowed us to not only identify the fatty acid that was attached to the hydroperoxide, but also the conformation of the double bonds. These assignments were performed for oleic, linoleic and $\alpha$-linolenic acid. For oleic and linoleic acid methyl ester hydroperoxides, different geometric isomers (cis/trans) were annotated. The $\alpha$-linolenic acid hydroperoxides were assigned based on hydroperoxide position (inner vs. outer vs. cyclised). The OOH-signals of the oxidised methyl esters all displayed resonances between $\delta 11.5-10.5$ ppm. For oleic acid, two peak-clusters were sufficiently base-line separated to be excited by a band-selective 1D NOESY pulse sequence. The high and low field signals respectively corresponded to cis and trans-isomers. In a similar way, cis-trans- and trans-trans-isomers could be distinguished for the linoleic acid methyl ester. We were not able to further elucidate whether the hydroperoxides were attached to the 8- and 11-positions for oleic acid or the 9and 13-positions for linoleic acid, since the chemical shift of the allylic protons (next to the hydroperoxide) was not significantly influenced by the position in the fatty acid alkyl chain. In the case of $\alpha$-linolenic acid methyl esters, the major distinction between the hydroperoxides formed by autoxidation, was not the isomeric configuration of the double bonds, but rather the position of the hydroperoxide moiety on the chain. The hydroperoxides were either formed on the 'inside' or 'outside' of the three double bonds. The 'inner'-isomers can cyclise internally, which generates additional cyclised hydroperoxides (Frankel, 2005). These cyclization mechanisms are inhibited by $\alpha$-tocopherol (Frankel, 2005) which enabled us to identify the 'inner'-isomers and cyclised-isomers, since these were only present in samples without $\alpha$-tocopherol.

Since the above-described analyses were done on the methyl esters, we needed to translate this to the triglycerides. To this end, we oxidised model triglycerides that consist of only one type of fatty acid (O, LA, $\alpha$-Ln) and compared their ${ }^{1} \mathrm{H}$ NMR spectra with those of the corresponding methyl esters. The spectra of hydroperoxides in triglycerides were almost 
identical to those in methyl esters. The chemical shifts of these signals did not significantly change, which enabled straightforward assignments of hydroperoxides signals originating from oxidised fatty acids in their esterified triglyceride form. In Figure 2.2 and Table 2.1 the assignments of hydroperoxides signals from oxidised fatty acids are summarised.
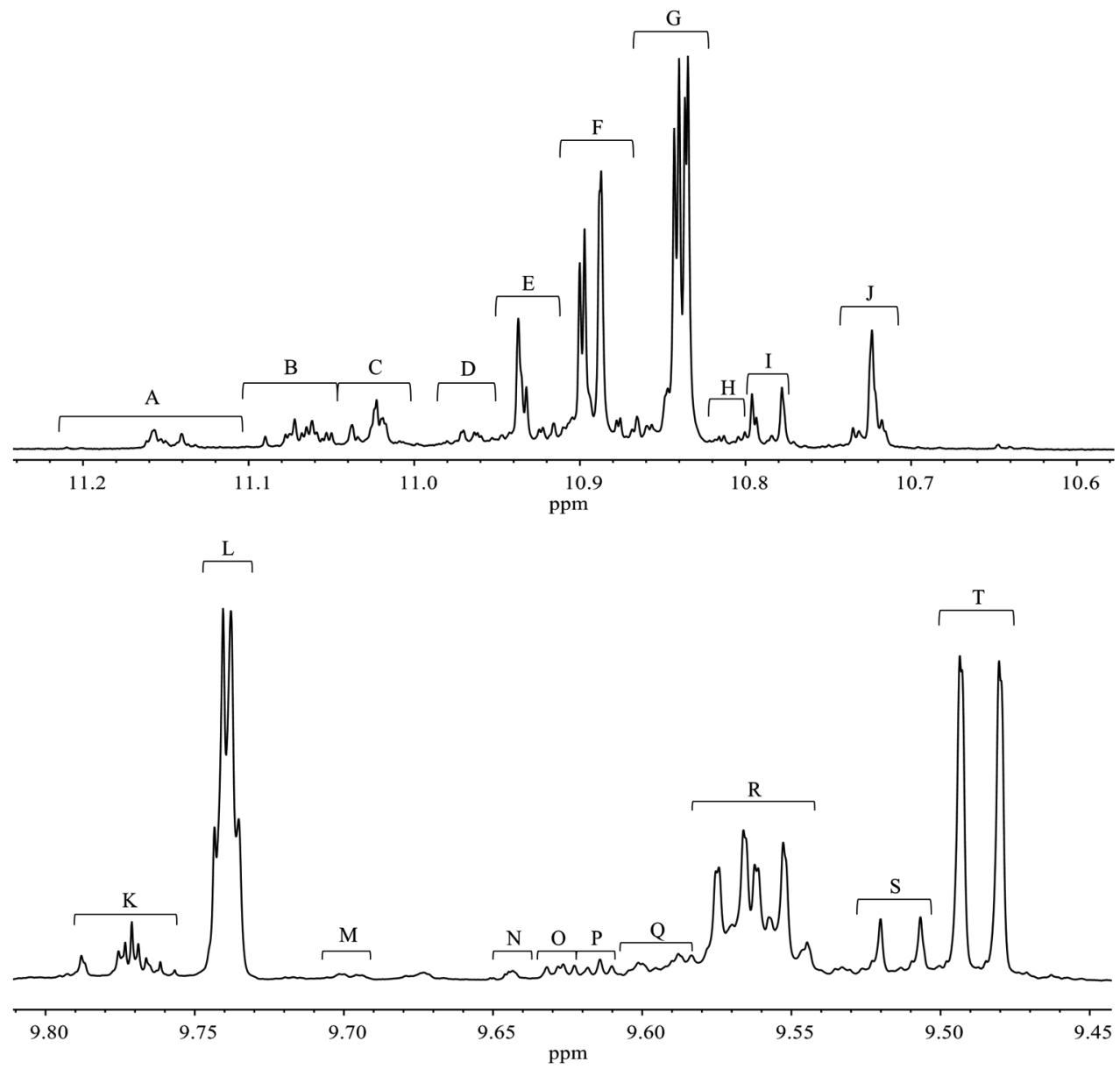

Figure 2.2. ${ }^{1} \mathrm{H}$ NMR spectra obtained with band selective excitation, with assignment coding, according to Table 2.1. The sample contained $25 \%$ oxidised oil in $75 \% 5: 1 \mathrm{CDCl}_{3}: \mathrm{DMSO}-\mathrm{d}_{6}$. Top: Hydroperoxide region $(\delta 11.24-$ $10.57 \mathrm{ppm}$ ); bottom: Aldehyde region ( $\delta 9.81-9.44 \mathrm{ppm})$. 


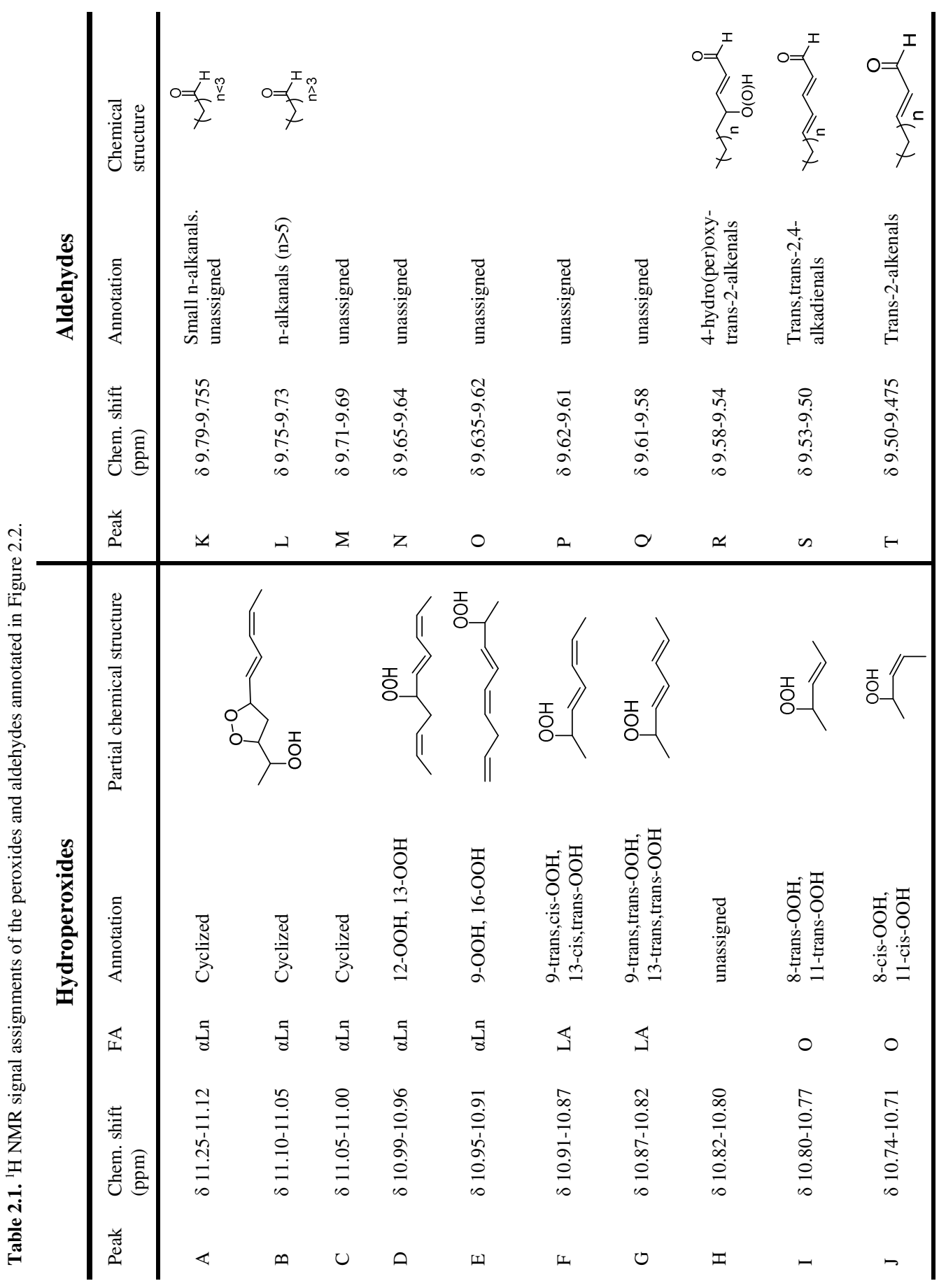




\subsubsection{Quantification of hydroperoxides and aldehydes}

For samples with high concentrations of oxidised lipids ( $>>1 \mathrm{mmol} / \mathrm{kg}$ ), the hydroperoxide, aldehyde and triglyceride signals can all be excited by a single pulse experiment with a sufficient signal-to-noise ratio. For such samples, the methylene signals at 4.4-4.0 ppm were used as an internal reference to quantify the total amount of hydroperoxides and aldehydes in $\mathrm{mmol} / \mathrm{kg}$ in a straightforward manner by using Equation 1. Samples with low amounts of oxidised lipids required the use of band selective experiments to acquire hydroperoxide and aldehyde ${ }^{1} \mathrm{H}$ NMR signals with sufficient sensitivity. For their quantification, single pulse experiments were used to acquire the methylene signals. To account for the different acquisition schemes of these two spectra, we implemented an empirical correction factor. This 'SelFactor' was determined by comparing signal intensities of spectra acquired by respectively single pulse and band-selective excitation for samples with high levels of oxidation products (Berger, 1997). In the calculations of concentrations of oxidation products, according to Equation 2, we also accounted for differences in excitation pulse angle, receiver gain and number of scans between the single pulse and band selective experiments. The band-selective and single-pulse experiments were recorded with a recycle delay of 5 seconds, the SelFactor also incorporated the effect of partial saturation by comparison of signal intensities of spectra recorded under fully relaxed conditions (recycle delay of 30 seconds).

The accuracy of the introduced correction factors in the ${ }^{1} \mathrm{H}$ NMR approach on hydroperoxides was verified by comparison of results with the classical PV titration method. Linear regression between the total hydroperoxide values obtained by NMR and PV methods resulted in an off-set bias and proportional bias that were not statistically significant (see Supporting Info, Table S2.1). For ${ }^{1} \mathrm{H}$ NMR band-selective measurements performed within five minutes, the limits of detection and quantification for individual hydroperoxide and aldehydes were respectively 0.01 and $0.03 \mathrm{mmol} / \mathrm{kg}$ (see Table 2.2). This is comparable to the conventional and more cumbersome PV method, which only measures total hydroperoxide concentration.

We also compared the aldehyde concentrations determined by ${ }^{1} \mathrm{H}$ NMR with the headspace GC method, which is commonly used in shelf-life monitoring of food emulsions since it measures a perceivable volatile and rancid secondary oxidation product. Figure 2.3 shows a good correlation $\left(\mathrm{R}^{2}=0.93\right)$ between hexanal semi-quantified in the head space of a freezethawed mayonnaise by GC-MS and the total n-alkanal concentrations as quantified directly in the extracted oil phase by ${ }^{1} \mathrm{H}$ NMR. The linear relationship is in line with vegetable oil being a near ideal solvent for hexanal, with a gas-oil partitioning coefficient $\mathrm{K} \approx 1 \cdot 10^{-3}$ (Landy et al., 2007). It is important to note that hexanal concentrations cannot be determined by ${ }^{1} \mathrm{H}$ NMR due to signal overlap with other n-alkanals $(n>5)$, which likely explains the small 
offset on the horizontal axis. The summation of $n$-alkanal levels as determined by ${ }^{1} \mathrm{H}$ NMR also partially explains the proportional bias versus hexanal levels determined in the headspace. Another factor that can explain the proportional bias is the elevated measurement temperature for the headspace GC-MS methods compared to the ambient condition for ${ }^{1} \mathrm{H}$ NMR. The low gas-oil partitioning coefficient $K$ implies that most hexanal is still dissolved in the oil layer and only a small amount is present in the headspace. This makes the GC method very prone to subtle variations in $K$, whereas this hardly impact on concentrations as determined by the ${ }^{1} \mathrm{H}$ NMR method. Furthermore, the headspace GC method assumes that the gas-matrix partition coefficient does not change during oxidation and therefore ideally requires the analyst to re-establish the gas-matrix coefficients of each analyte at different oxidation stages (Savary, Guichard, Doublier, \& Cayot, 2006). In summary, even though the methods intrinsically differ in what and how they measure alkanals, they both show the same trend, with the main advantage of the ${ }^{1} \mathrm{H}$ NMR method is that an absolute quantification of $\mathrm{n}$-alkanals in the oil phase can be provided, not biased by variation in partition coefficients.

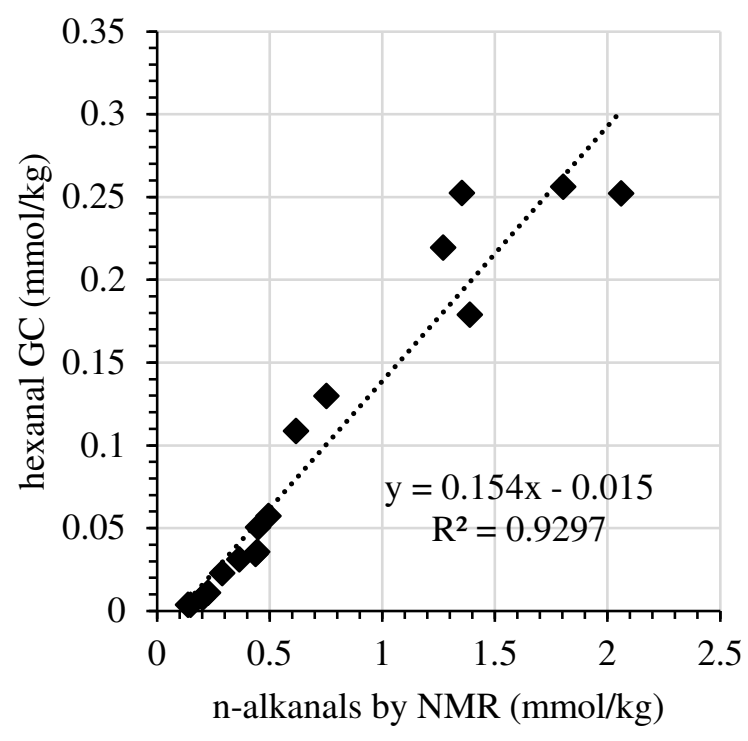

Figure 2.3. Comparison of hexanal concentrations in the headspace vials with freeze-thawed mayonnaise measured by headspace GC after 30 min equilibration at $60^{\circ} \mathrm{C}$ with concentrations of $n$-alkanals in oil extracted from mayonnaise stored in vials determined by ${ }^{1} \mathrm{H}$ NMR at ambient temperature.

The precision of the method for the assessment of hydroperoxides and aldehydes was determined by performing duplicate experiments over different measurement days and concentration levels and expressed in terms of repeatability and reproducibility (respectively 
between- and within-day variation) by ANOVA (Table 2.2). Pooled relative standard deviations of repeatability $\left(\mathrm{RSD}_{\mathrm{r}}\right)$ and within-laboratory reproducibility $\left(\mathrm{RSD}_{\mathrm{R}}\right)$ were respectively $5.7 \%$ and $5.9 \%$. The repeatability and within-laboratory reproducibility variations are close, indicating that the method is stable over time. The aldehyde concentrations, which are closest to the limit of quantification, have the highest relative standard deviations, but are still within the limits to allow for quantitative profiling studies.

Table 2.2. Overview of the validation results for hydroperoxide and aldehyde quantification using band-selective excitation (32 scans).

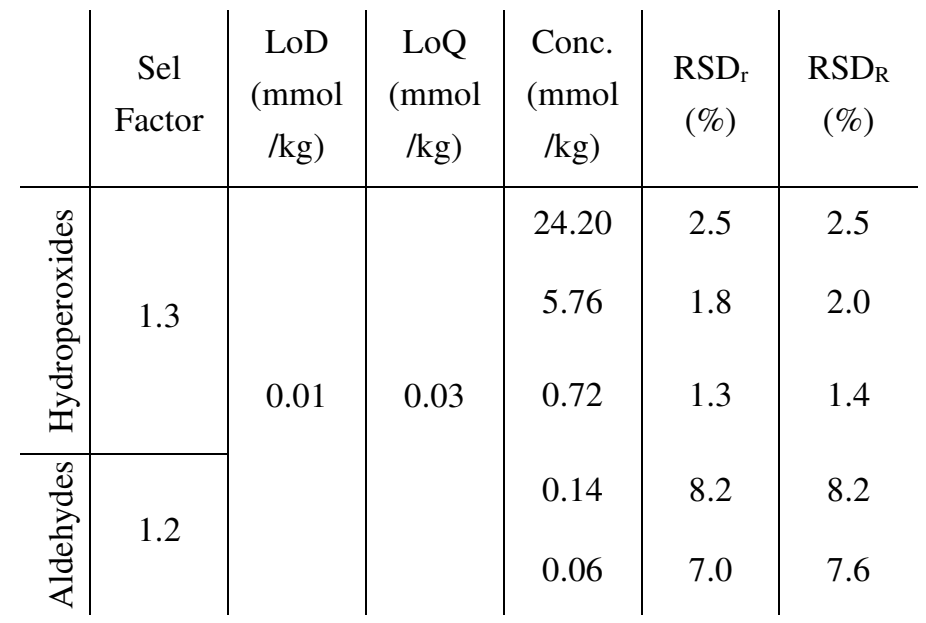

\subsubsection{Application: assessment of oxidative stability of mayonnaise}

We used Principal Component Analysis (PCA) to assess the impact of storage temperature on oxidative mechanisms in a food emulsion. A conventional mayonnaise formulation was used, stored at two different temperatures: room temperature $\left(22^{\circ} \mathrm{C}\right)$ for 200 days and elevated temperature $\left(50^{\circ} \mathrm{C}\right)$ for 53 days. The oxidation products formed in these mayonnaise samples were quantified by ${ }^{1} \mathrm{H}$ NMR, after which PCA was used to map compositional variations in time.

The first three principal components explained $97 \%$ of the total variation in the model. Most of the variation was explained by PC 1 (75.9\%), followed by PC 2 (18.5\%) and PC 3 (2.5\%). PC 1 captured the overall variation of primary and secondary lipid oxidation products over time. Here, all oxidation products showed positive loadings (see Supporting Info, Figure S2.2). Since the variation in PC1 could be explained by the overall time-dependence of primary and secondary lipid oxidation, this principal component could not discriminate between different oxidation mechanisms. For PC 2 (Figure 2.4B), the positive loadings could 
be assigned to the major hydroperoxides, while the negative loadings primarily corresponded to the aldehydes. This difference prompted us to map formation of hydroperoxides versus aldehydes (Figure 2.5A). At elevated T, both hydroperoxides and aldehydes were formed. However, at ambient $\mathrm{T}$, the formation of hydroperoxides exceeds the formation of aldehydes. The enhanced generation of aldehydes at elevated $\mathrm{T}$ may be attributed to instability of hydroperoxides. Consequently, this may bias the translation of accelerated shelf-life experiments performed at elevated temperature to ambient conditions.

A

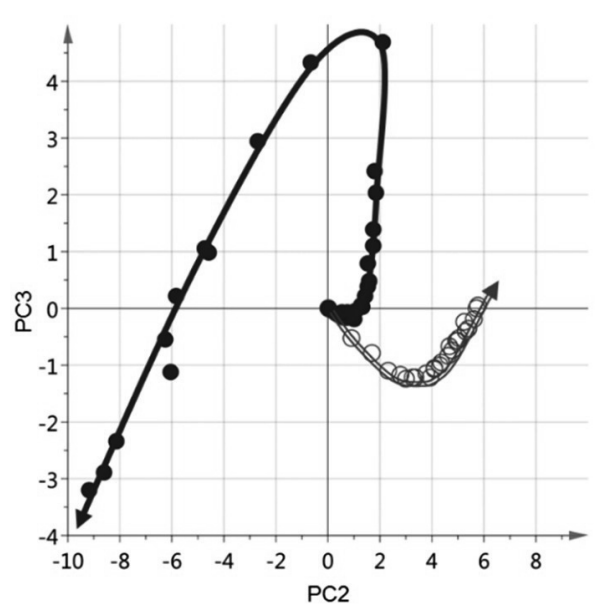

B

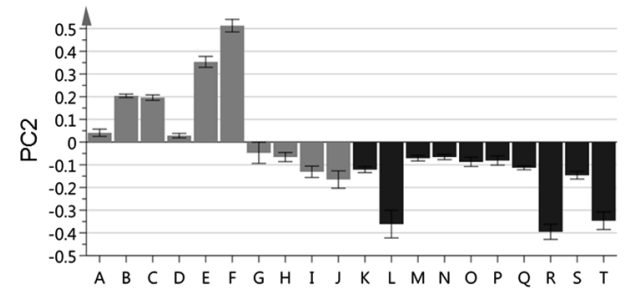

$\mathrm{C}$

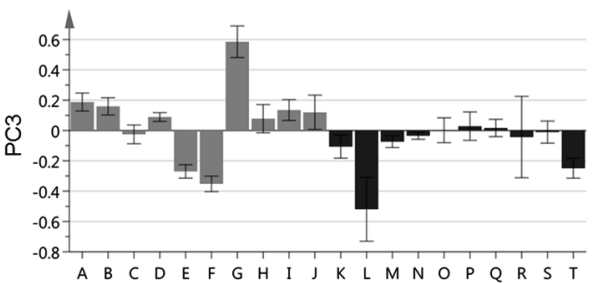

Figure 2.4. Oxidation product profiles of mayonnaise stored at two different temperatures and followed in time. A) Score plot of PC 2 versus PC 3. The arrows in the score plots indicate the trajectory through time, the base of the arrow is timepoint zero, the arrowhead is located at the last timepoint. Samples at elevated $($ ) and ambient ( O) temperature are respectively stored for 53 and 203 days. B/C) Loading plots of PC2/PC3, where grey bars represent hydroperoxides and black bars aldehydes. A-T correspond to the annotations in Table 2.1.

For PC3 (2.5\%), the loading plot (Figure 2.4C) showed opposite loadings for different lipid hydroperoxides isomers (F vs. G, A-D vs. E). To verify and visualise whether storage temperature had an impact on isomeric preferences of hydroperoxide formation, we plotted the trans-trans-LA-OOH versus cis-trans-LA-OOH for both storage conditions (Figure 5B). At ambient T, relatively small amounts of trans-trans-LA-OOH were formed, whereas at elevated $\mathrm{T}$ these trans-trans-LA-OOH levels exceed those of cis-trans-LA-OOH. This could be attributed to temperature induced cis-trans isomerization of the native cis bonds in LA. Although this isomerization reaction is known to occur at elevated temperatures, it has not been well appreciated that this may also impact the formation of different primary oxidation products. Furthermore, we conjecture that the formation of volatile secondary oxidation 
products may be impacted by storage at accelerated elevated temperature conditions, which may bias translation of results to ambient conditions.

A

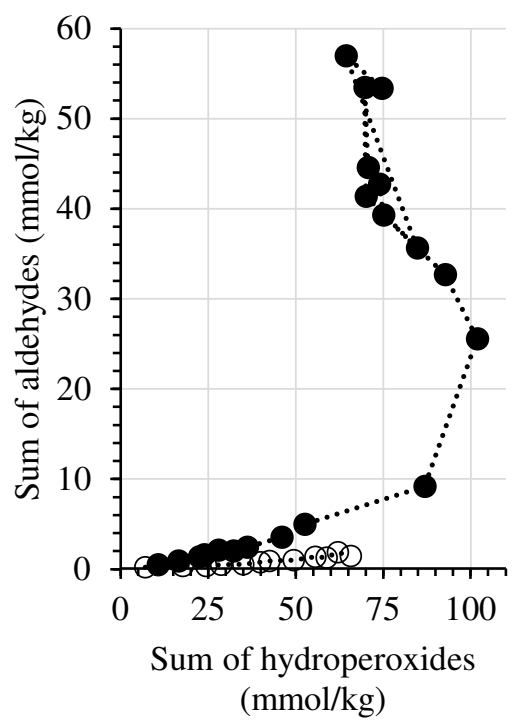

B

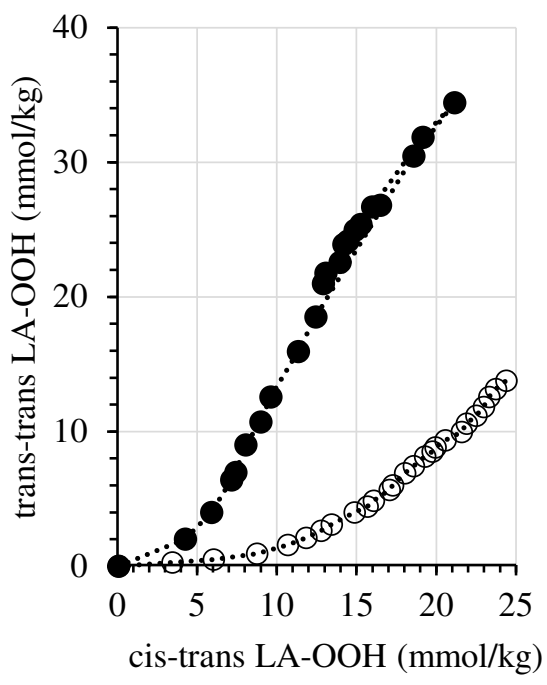

Figure 2.5. Map of oxidation trajectories at elevated $(-$ and ambient $(\mathrm{O})$ temperature, respectively stored for 53 and 203 days. (A) Plot of aldehydes vs. hydroperoxides (PC2 visualised) (B) plot of trans-trans-LA-OOH vs. cis-trans-LA-OOH (PC3 visualised).

In this work, the main purpose of the unsupervised explorative modelling by PCA was to demonstrate the quality and potential of quantitative profiling of primary and secondary oxidation products by ${ }^{1} \mathrm{H}$ NMR. In future work, we intend to exploit the longitudinal nature of the oxidation profiles acquired during food emulsion shelf-life tests by more sophisticated multivariate analysis techniques, such as batch Partial Least Squares (PLS) (Smilde et al., 2010; Wold, Kettaneh, Fridén, \& Holmberg, 1998). Furthermore, we expect to deploy multivariate ANOVA (Smilde et al., 2010) and multilevel multivariate approaches to disentangle sources of variation in longitudinally acquired oxidation profiles such as storage conditions (temperature, $\mathrm{O}_{2}$ pressure) and (pro- and anti-oxidant) compositions of the food emulsions (Antti et al., 2002; Jonsson et al., 2006; Nomikos \& MacGregor, 1995). 


\subsection{Conclusion}

${ }^{1} \mathrm{H}$ NMR can be used as a convenient and fast method to assess lipid oxidation products in food emulsions. By using band selective NOESY and TOCSY experiments, up to nine primary lipid oxidation products can be identified at level of common fatty acid types, cis/trans isomers and chain position of hydroperoxide moiety. Band-selective excitation allows quantification of hydroperoxides and aldehydes precisely $\left(\operatorname{RSD}_{\mathrm{R}}=5.9 \%\right)$ with a detection limit of $0.01 \mathrm{mmol} / \mathrm{kg}$ in less than five minutes. In comparison to conventional onedimensional methods, such as PV or headspace GC, ${ }^{1} \mathrm{H}$ NMR profiling provides both a sensitive and simultaneous quantification of primary and secondary oxidation products. Explorative modelling of the quantitative ${ }^{1} \mathrm{H}$ NMR profiles of primary and secondary oxidation products reveals the modulation of lipid oxidation mechanism by temperature.

\subsection{Literature}

Adams, R. W., Holroyd, C. M., Aguilar, J. A., Nilsson, M., \& Morris, G. A. (2013). "Perfecting" WATERGATE: clean proton NMR spectra from aqueous solution. Chemical Communications, 49(4), 358-360.

Antti, H., Bollard, M. E., Ebbels, T., Keun, H., Lindon, J. C., Nicholson, J. K., \& Holmes, E. (2002). Batch statistical processing of $1 \mathrm{H}$ NMR-derived urinary spectral data. Journal of Chemometrics, 16(8-10), 461-468.

Berger, S. (1997). NMR techniques employing selective radiofrequency pulses in combination with pulsed field gradients. Progress in Nuclear Magnetic Resonance Spectroscopy, 30(3), 137-156.

Berton-Carabin, C. C., Ropers, M.-H., \& Genot, C. (2014). Lipid Oxidation in Oil-in-Water Emulsions: Involvement of the Interfacial Layer. Comprehensive Reviews in Food Science and Food Safety, 13(5), 945-977.

Budilarto, E. S., \& Kamal-Eldin, A. (2015). The supramolecular chemistry of lipid oxidation and antioxidation in bulk oils. Eur J Lipid Sci Technol, 117(8), 1095-1137.

Coupland, J. N., \& McClements, D. J. (1996). Lipid oxidation in food emulsions. Trends in Food Science \& Technology, 7(3), 83-91.

Dugo, G., Rotondo, A., Mallamace, D., Cicero, N., Salvo, A., Rotondo, E., \& Corsaro, C. (2015). Enhanced detection of aldehydes in Extra-Virgin Olive Oil by means of band selective NMR spectroscopy. Physica A: Statistical Mechanics and its Applications, 420, 258-264.

Folch, J., Lees, M., \& Sloane Stanley, G. H. (1957). A simple method for the isolation and purification of total lipides from animal tissues. J Biol Chem, 226(1), 497-509.

Frankel, E. N. (2005). Hydroperoxide formation. In Lipid Oxidation (2nd ed., pp. 25-50): Elsevier.

Frankel, E. N., Hu, M.-L., \& Tappel, A. L. (1989). Rapid headspace gas chromatography of hexanal as a measure of lipid peroxidation in biological samples. Lipids, 24(11), 976. 
Frankel, E. N., \& Meyer, A. S. (2000). The problems of using one-dimensional methods to evaluate multifunctional food and biological antioxidants. Journal of the Science of Food and Agriculture, 80(13), 1925-1941.

Ghorbani Gorji, S., Smyth, H. E., Sharma, M., \& Fitzgerald, M. (2016). Lipid oxidation in mayonnaise and the role of natural antioxidants: A review. Trends in Food Science \& Technology, 56, 88-102.

Guillen, M. D., \& Goicoechea, E. (2009). Oxidation of corn oil at room temperature: Primary and secondary oxidation products and determination of their concentration in the oil liquid matrix from $1 \mathrm{H}$ nuclear magnetic resonance data. Food Chemistry, 116(1), 183-192.

Guillén, M. D., \& Ruiz, A. (2008). Monitoring of heat-induced degradation of edible oils by proton NMR. European Journal of Lipid Science and Technology, 110(1), 52-60.

Ibargoitia, M. L., Sopelana, P., \& Guillen, M. D. (2014). (1)H Nuclear Magnetic Resonance monitoring of the degradation of margarines of varied compositions when heated to high temperature. Food Chem, 165, 119-128.

ISO 3960:2017. (2017). In Animal and vegetable fats and oils -- Determination of peroxide value -- Iodometric (visual) endpoint determination (pp. 10). Geneva: International Organization for Standardization.

ISO 6885:2016. (2016). In Animal and vegetable fats and oils -- Determination of anisidine value (pp. 7). Geneva: International Organization for Standardization.

Jacobsen, C. (1999). Sensory impact of lipid oxidation in complex food systems. European Journal of Lipid Science and Technology, 101(12), 484-492.

Jonsson, P., Stenlund, H., Moritz, T., Trygg, J., Sjöström, M., Verheij, E. R., . . Antti, H. (2006). A strategy for modelling dynamic responses in metabolic samples characterized by GC/MS. Metabolomics, 2(3), 135-143.

Laghi, L., Picone, G., \& Capozzi, F. (2014). Nuclear magnetic resonance for foodomics beyond food analysis. 59.

Laguerre, M., Bayrasy, C., A., P., Weiss, J., Mcclements, D. J., Lecomte, J., . . Villeneuve, P. (2015). What makes food antioxidatns in lipid-based systems? The enxt theories beyond the polar paradox. Critical Reviews in Food Science and Nutrition, 55, 183201.

Laguerre, M., Bily, A., Roller, M., \& Birtic, S. (2017). Mass Transport Phenomena in Lipid Oxidation and Antioxidation. Annual Review of Food Science and Technology, 8(1), 391-411.

Landy, P., Pollien, P., Rytz, A., Leser, M. E., Sagalowicz, L., Blank, I., \& Spadone, J.-C. (2007). Model Studies on the Release of Aroma Compounds from Structured and Nonstructured Oil Systems Using Proton-Transfer Reaction Mass Spectrometry. Journal of Agricultural and Food Chemistry, 55(5), 1915-1922.

McClements, D., \& Decker, E. (2000). Lipid oxidation in oil-in-water emulsions: Impact of molecular environment on chemical reactions in heterogeneous food systems. Journal of Food Science, 65(8), 1270-1282.

Melliou, E., Magiatis, P., \& Killday, K. B. (2015). A New Ultra Rapid Screening Method for Olive Oil Health Claim Evaluation Using Selective Pulse NMR Spectroscopy. In Magnetic Resonance in Food Science: Defining Food by Magnetic Resonance (pp. 84-92): The Royal Society of Chemistry.

Miller, J. N., \& Miller, J. C. (2010). Statistics and Chemometrics for Analytical Chemistry (6th ed.): Pearson Education Limited. 
Mozuraityte, R., Rustad, T., \& Storrø, I. (2008). The Role of Iron in Peroxidation of Polyunsaturated Fatty Acids in Liposomes. Journal of Agricultural and Food Chemistry, 56, 537-543.

Nomikos, P., \& MacGregor, J. F. (1995). Multivariate SPC Charts for Monitoring Batch Processes. Technometrics, 37(1), 41-59.

Rastrelli, F., Schievano, E., Bagno, A. \& Mammi, S. . (2009). NMR quantification of trace components in complex matrices by band-selective excitation with adiabatic pulses. . Magnetic Resonance in Chemistry, 47, 868-872.

Savary, G., Guichard, E., Doublier, J.-L., \& Cayot, N. (2006). Mixture of aroma compounds: Determination of partition coefficients in complex semi-solid matrices (Vol. 39).

Skiera, C., Steliopoulos, P., Kuballa, T., Holzgrabe, U., \& Diehl, B. (2012a). 1H-NMR Spectroscopy as a New Tool in the Assessment of the Oxidative State in Edible Oils. Journal of the American Oil Chemists' Society, 89, 1383-1391.

Skiera, C., Steliopoulos, P., Kuballa, T., Holzgrabe, U., \& Diehl, B. (2012b). 1H NMR approach as an alternative to the classical p-anisidine value method. European Food Research and Technology, 235, 1101-1105.

Smilde, A. K., Westerhuis, J. A., Hoefsloot, H. C., Bijlsma, S., Rubingh, C. M., Vis, D. J., . . . van der Greef, J. (2010). Dynamic metabolomic data analysis: a tutorial review. Metabolomics, 6(1), 3-17.

van Duynhoven, J., van Velzen, E., \& Jacobs, D. M. (2013). Quantification of Complex Mixtures by NMR. In Annual Reports on NMR Spectroscopy (Vol. 80, pp. 181-236).

Wold, S., Kettaneh, N., Fridén, H., \& Holmberg, A. (1998). Modelling and diagnostics of batch processes and analogous kinetic experiments. Chemometrics and Intelligent Laboratory Systems, 44(1), 331-340. 


\subsection{Supporting information}

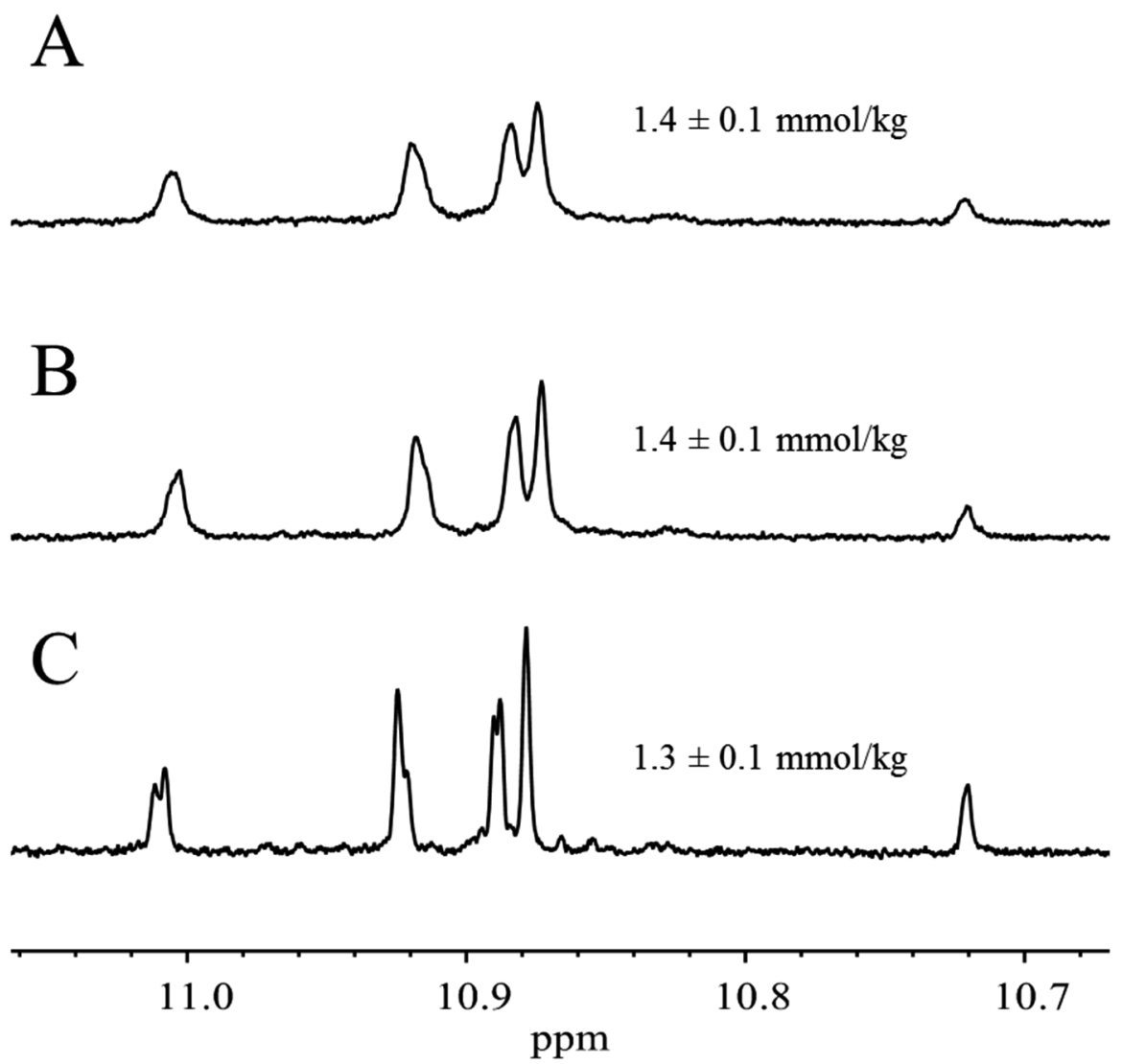

Figure S2.1. Hydroperoxide regions of the ${ }^{1} \mathrm{H}$ NMR spectra $(700 \mathrm{MHz})$ of extracts obtained from an oxidised mayonnaise after $(\mathrm{A}, \mathrm{B})$ chloroform-methanol extraction and $(\mathrm{C})$ freeze-thaw extraction.

Table S2.1. Results of linear regression between the total hydroperoxide values obtained by ${ }^{1} \mathrm{H}$ NMR and the PV method.

\begin{tabular}{ll}
\hline Measure & Value \\
\hline Intercept $\pm 0.5 . \mathrm{CI}_{\text {intercept }}$ & $0.8152 \pm 0.8573$ \\
Max. off-set bias & 1.6725 \\
Slope $\pm 0.5 . \mathrm{CI}_{\text {slope }}$ & $1.1275 \pm 0.1870$ \\
Max. proportional bias & 0.3145 \\
\hline
\end{tabular}


A

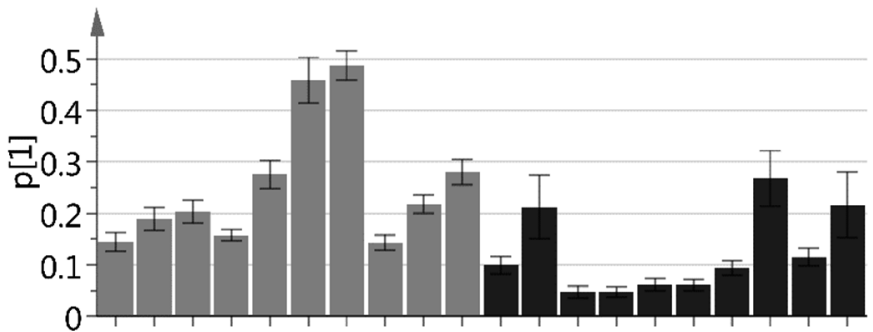

A B C D E F G H I J K L M N P Q R S T

$\mathrm{B}$

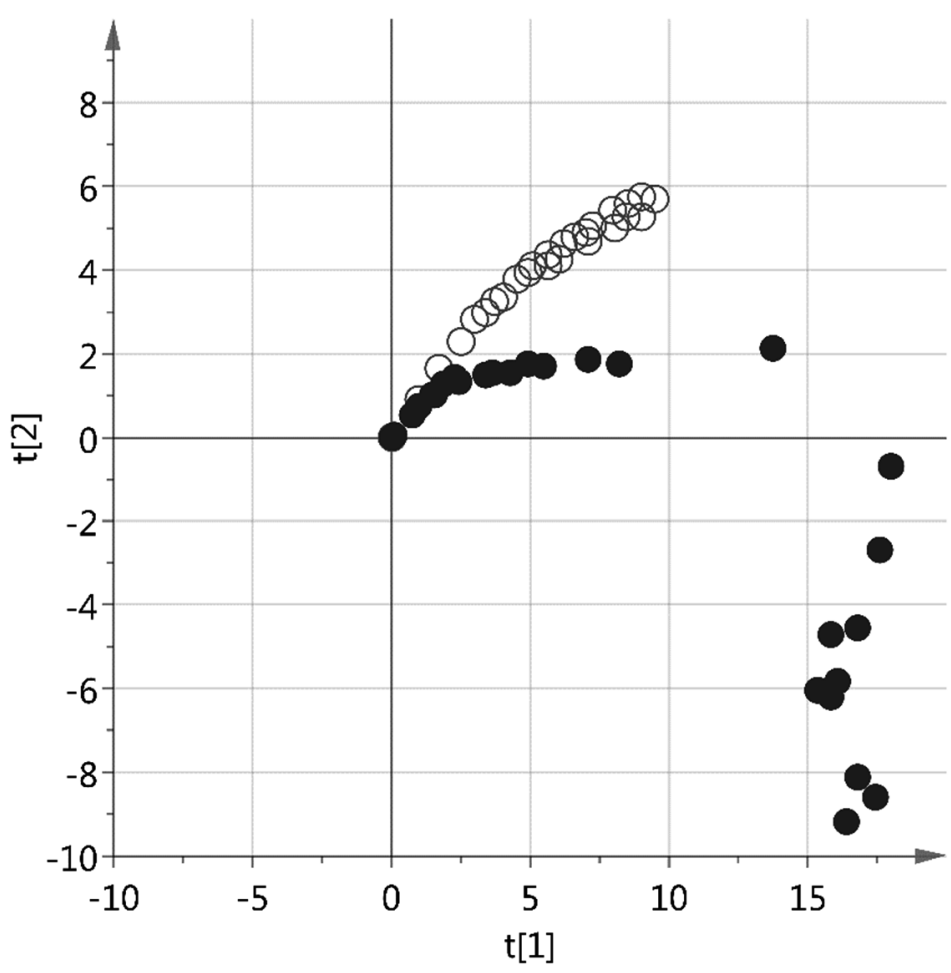

Figure S2.2. Oxidation product profiles of mayonnaise stored at two different temperatures and followed in time. A) Loading plots of PC1, where grey bars represent hydroperoxides and black bars aldehydes. A-T correspond to the annotations in Table 2.1. B) Score plot of PC 1 versus PC 2. Samples at elevated () and ambient (O) temperature are respectively stored for 53 and 203 days. 




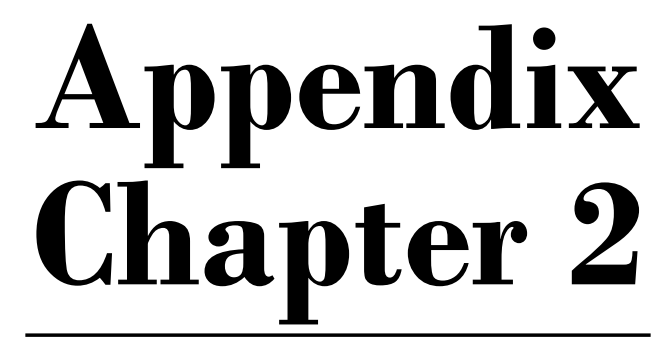

\section{Quantitative ${ }^{1} \mathbf{H}$ NMR analysis of food lipid hydroperoxides and aldehydes}

Based on: Merkx, D. W. H., Boerkamp, V. J. P., Hennebelle, M., \& van Duynhoven, J. P. M., Quantitative ${ }^{1} \mathrm{H}$ NMR analysis of food lipid hydroperoxides and aldehydes. In S.S. Anderson (Ed.), Multidimensional characterization of dietary lipids, New York City:

Springer, to be published 


\begin{abstract}
${ }^{1} \mathrm{H}$ NMR spectroscopy enables rapid and sensitive one-shot quantification of both lipid hydroperoxides and aldehydes as primary and secondary oxidation products in lipid-based food ingredients and products. The method is straightforward and can be applied to any type of vegetable oils and oil-extracts but requires access to a commercial high-field NMR instrument. The sensitivity is 3 -fold improved by using a band-selective pulse excitation, which results in a limit of detection below $0.1 \mathrm{mmol} / \mathrm{kg}$ for lipid hydroperoxides when using a $600 \mathrm{MHz}$ NMR equipped with a cryoprobe. Here, we describe a step-by-step approach how to get from an oil (extract) to quantitative hydroperoxide and aldehyde content in one-go.
\end{abstract}




\section{A-2.1. Introduction}

By monitoring only a single class of oxidation products, the extent of lipid oxidation could be greatly underestimated (Schaich, 2020). Therefore, to accurately assess the oxidative state of foods, both the primary and secondary oxidation products should be quantified. Various techniques have been put forward to track primary oxidation using the peroxide value (PV), including iodometric titration and colorimetric detection of $\mathrm{Fe}(\mathrm{II})$ oxidation (Dobarganes \& Velasco, 2002). For the analysis of secondary oxidation, thiobarbituric acid reactive substances (TBARS) and the p-Anisidine Value (p-AV) are frequently used (Barriuso, Astiasarán, \& Ansorena, 2013). All these methods determine only single specie type (peroxides or specific aldehydes) and suffer from high labor intensity, low sensitivity, poor reproducibility, and distortion by contaminants (Antolovich, Prenzler, Patsalides, McDonald, \& Robards, 2002; Barriuso et al., 2013; Dobarganes \& Velasco, 2002). Analysis of oxidation products with chromatographic methods (GC; HPLC) mitigates most issues, as it enables identification of a wide range of lipid hydroperoxides $(\mathrm{LOOH})$ or volatiles with high sensitivity. These methods, however, suffer from a low throughput and require pure standards for accurate quantification (Dobarganes \& Velasco, 2002). In recent years, Proton Nuclear Magnetic Resonance $\left({ }^{1} \mathrm{H}\right.$ NMR) spectroscopy has been used increasingly for the analysis of food lipid oxidation products (Goicoechea \& Guillen, 2010; Guillen \& Goicoechea, 2009; Guillén \& Ruiz, 2008; Merkx, Hong, Ermacora, \& van Duynhoven, 2018; Skiera, Steliopoulos, Kuballa, Holzgrabe, \& Diehl, 2012a, 2012b). It is reproducible, easy to automate, high-throughput, and independent of pure standards for quantification. The method can be applied directly on vegetable oil samples or after lipid extraction for more complex food samples. The addition of DMSO-d6 to $\mathrm{CDCl}_{3}$ as solvent allows for accurate quantification of the hydroperoxides (Skiera et al., 2012a). Sensitivity issues due to dynamic range limitations can be overcome using selective pulse gradients (Merkx et al., 2018). This enables ${ }^{1} \mathrm{H}$ NMR measurements of less than 3 minutes per sample (600 $\mathrm{MHz} \mathrm{NMR}$ instrument equipped with a cryoprobe) with a limit of detection of $0.1 \mathrm{mmol} \mathrm{kg}^{-1}$ for LOOH, which is comparable or even outperforms classical titration and UV-methods. Besides its high through-put and sensitivity, ${ }^{1} \mathrm{H}$ NMR also presents the advantage to allow the simultaneous quantification of both primary and secondary oxidation products. Next to the hydroperoxides, non-volatile aldehydes are indeed quantified in the same run. The quantification of individual hydroperoxides and aldehydes is possible without the addition of internal standard by using the triglyceride peak as a reference and can potentially provide valuable information for reaction pathway elucidation (Merkx et al., 2018). Here, we describe a step-by-step protocol to quantify hydroperoxides and aldehydes in oils (or lipid extracts) using ${ }^{1} \mathrm{H}$ NMR. 


\section{A-2.2. Materials}

\section{A-2.2.1. Equipment}

This method requires a high-field NMR system, which implies a Larmor frequency of ${ }^{1} \mathrm{H}$ nuclei of at least $400 \mathrm{MHz}(9.4 \mathrm{~T})$. It also requires a console which can perform selective gradient pulses, which is common for modern commercial NMR spectrometers. A cryogenic probe head will allow for short measurement times but is not a necessity. Optional, but highly recommended, is the use of an automatic sample changer, to allow a large sequence of samples to be measured without intervention of the analyst.

\section{A-2.2.2. Chemicals}

- $\quad$ Deuterated chloroform $(\mathrm{CDCl} 3)+0.03 \%$ TMS, 99.80\% D, CAS [865-49-6]

- Deuterated dimethyl sulfoxide (DMSO-d6), 99.80\% D, CAS [2206-27-1]

- Optional: deuterated molecular sieves, 99.80\%, CAS [N1311]

- $5 \mathrm{~mm}$ NMR tubes

- Glass laboratory bottle, $100-500 \mathrm{~mL}$

- Disposable $1.5 \mathrm{ml}$ PP micro-centrifuge tubes

\section{A-2.3. Method}

\section{A-2.3.1. NMR-solvent preparation}

i. Transfer $50 \mathrm{~mL}$ of deuterated chloroform $\left(\mathrm{CDCl}_{3}\right)$ to a glass laboratory bottle.

ii. Add $10 \mathrm{~mL}$ of deuterated dimethylsulfoxide (DMSO- $\left.\mathrm{d}_{6}\right)$.

iii. Optional: add $100 \mathrm{mg}$ deuterated molsieves. This step is advised if the solvent is stored for longer than a week. If molsieves are not available, ensure the solvent attracts as minimal water as possible.

iv. Close the bottle and mix the solution manually for at least 10 seconds. The solution will warm up slightly.

v. Let the solution cool down prior to first use.

vi. When using the solution, swirl the bottle briefly ( $\sim 3$ seconds)

\section{A-2.3.2. Sample preparation}


This method requires oil or fat, which needs to be devoid of water as this might disturb accurate and precise NMR quantification. For example, oil can be used directly; for high-oil emulsions such as mayonnaise use a freeze-thaw induced phase separation to separate the oil; other types of samples and emulsions may require a lipid extraction first, such as Folch or Bligh and Dyer.

i. Transfer $150 \mu \mathrm{L}$ of oil or $\sim 135 \mathrm{mg}$ fat to a small container, e.g., a $1.5 \mathrm{~mL}$ microcentrifuge tube. These are the recommended and optimal quantities, note that the minimal suggested amount is $50 \mu \mathrm{L}$ oil or $45 \mathrm{mg}$ fat.

ii. To this, add $450 \mu \mathrm{L}$ NMR-solvent $\left(5: 1 \mathrm{CDCl}_{3}\right.$ :DMSO-d 6 )

iii. Mix the solution by closing the container and gentle tumbling of the container (2 seconds) to ensure that the solution is clear, and no phase separation is observed (2 seconds).

iv. Transfer the full solution $(\sim 600 \mu \mathrm{L})$ to a $5 \mathrm{~mm}$ NMR tube using a glass Pasteur pipette. Cap the NMR tube properly.

\section{A-2.3.3. NMR acquisition}

The following steps describe manual operation of a Bruker NMR spectrometer. Note that most, if not all steps, can be done fully automatically in most modern NMR facilities. The commands as used in the Bruker Topspin software are displayed in brackets, for other data acquisition software a skilled operator can identify similar commands.

Note that this protocol has been developed using TopSpin 3.6.1, operating a AVANCE III console with a cryoprobe on a $600 \mathrm{MHz}$ system. The suggested pulse values may therefore be different in your system and it is advised to always consult the NMR supervisor for the optimal setup.

i. Insert the NMR tube into the magnet.

ii. Set the internal temperature of the probe to $295 \mathrm{~K}$. [TE 295]

a. Wait until the sample temperature is stable (minimum 3 minutes) before continuing to step vii (shimming). Meanwhile, you can continue with steps iii to vi.

iii. Create a new experiment. [NEW]

iv. Select a single pulse proton experiment. [zg // zg30]

v. Tune and match the sample. [ATMA]

vi. Lock the sample to deuterated DMSO. [LOCK DMSO] 
a. Note that due to the presence of deuterated $\mathrm{CDCl} 3$, the system might lock wrongly in this step. If this happens, lock onto deuterated $\mathrm{CDCl} 3$ instead. [LOCK CDCL3]

Two experiments will be run after each other, a single pulse experiment to record the full spectrum (including the triglyceride peak for quantification) and a bandselective pulse experiment for the assessment of the oxidation products.

Single pulse experiment:

vii. Shim the sample. [TOPSHIM or GS]

a. Proper shimming is required to ensure sharp quantifiable peaks.

viii. Determine the length of the $90^{\circ}$ pulse. [PULSECAL]

ix. Set the relaxation delay to 5 seconds. [D1 5]

X. Set the acquisition time to 4 seconds. [AQ 4]

xi. Set the spectral width to $20 \mathrm{ppm}$. [SW 20]

xii. Set the offset to $6.175 \mathrm{ppm}$. [O1P 6.175]

xiii. Determine the ideal receiver gain. [RGA]

xiv. Set the number of scans to a minimum of 4. [NS 4]

Xv. Set the number of dummy scans to 2. [NS 2]

xvi. Run the experiment. [ZG]

a. Note if TopSpin reports 'receiver overflows', and the receiver gain is already at its lowest value, that the pulse length can be shortened further (possible in Cryoprobe-systems).

The band-selective pulse experiment can be created manually or by an interactive module in TopSpin.

Manual (skip steps xxvi - xxxv):

xvii. Make a new experiment [NEW] or increase the experiment number. [IEXPNO]

a. Ensure that the first experiment is not overwritten, as both spectra are required for the quantification.

xviii. Select a selective pulse sequence. [selgpse]

xix. Retrieve the powers. [GETPROSOL]

xx. Set the center of the selective pulse on the center of the desired region, e.g., at $\delta 10.5$ ppm . [CNST21 10.5]

xxi. Set the pulse length at the same value as in single pulse experiment. [e.g., P1 7.6]

xxii. Set the width of the band-selective pulse by changing the following:

a. SPDB2 15

b. P12 550

Note that lower SPDB2 and P12 increase the width of the band-selective pulse area. 
xxiii. Set the digitiser mode to digital. [DIGMOD DIGITAL]

xxiv. Set the zg-options to calculate the proper shaped pulse. [ZGOPTNS DCALC_SPOFFS]

$\mathrm{xxv}$. Set the correct gradient files.

a. GPNAM 1 SMSQ10.100

b. GPZ1 15

Optional: Use a REBURP-pulse instead of the default Gaussian inverse pulse for a sharper excitation. More detail can be found in Merkx et al.(Merkx et al., 2018) The pulse sequences for these selective pulses are available in the libraries of modern NMR spectrometers. TopSpin commands:
a. SPNAM2 Reburp.1000
b. P12 2000

Interactive (skip steps xvii - xxv):

xxvi. Open the single-pulse experiment.

xxvii. Locate the hydroperoxide and aldehyde regions, integrate [.INT] the area between 13 and 8 ppm, or tighter if possible.

xxviii. Click on the floppy disk with an 'a' next to it on the top of the integration module, select the option 'Save Regions to 'reg'.

xxix. Leave the integration module. [.SRET]

xxx. Locate and click the 'Acquire' tab on the top of your TopSpin interface.

xxxi. Click on the sub-tab 'More' and select 'Setup Selective 1D Expts.'.

xxxii. Click on 'Create Datasets'.

xxxiii. Select 'Selective Gradient $1 \mathrm{H}$ '.

a. Optional, change the shape of the pulse to Reburp.1000.

xxxiv. Press 'Accept'.

xxxv. In the next window, press 'cancel' to only create the data set and not start the acquisition yet.

Manual and interactive:

xxxvi. Set the relaxation delay to 5 seconds. [D1 5]

xxxvii. Set the acquisition time to 4 seconds. [AQ 4]

xxxviii. Set the spectral width to $20 \mathrm{ppm}$. [SW 20]

xxxix. Determine the ideal receiver gain automatically. [RGA] 
xl. Set the number of scans to a minimum of 16. [NS 16]

xli. Set the number of dummy scans to 4. [NS 4]

xlii. Run the experiment. [ZG]

\section{A-2.3.4. NMR pre-processing}

i. Make sure that the sise of the spectrum [SI] is equal to the sise of the Free Induction Decay (FID) [TD]. Note that the sise of the spectrum can be changed after acquisition, but the sise of the FID cannot.

ii. Use a Fourier-Transform on the FID spectrum. [FT]

a. Optionally, the Fourier Transform can be performed with an exponential window function with a line broadening of $0.3 \mathrm{~Hz}$ [LB 0.3 , followed by [EFP] to obtain smoother peaks.

iii. Correct the phase of spectrum manually [.PH] or automatically. [APK]

iv. Correct the baseline manually [.BASL] or automatically. [ABSN]

v. Calibrate the spectrum to have Trimethylsilane (TMS) at $0.00 \mathrm{ppm}$, either manually [.CAL] or automatically. [SREF]

\section{A-2.3.5. Integration}

Spectral integration is what allows us to quantify the oxidation products. In this protocol, the emphasis will be on the integration of three peak areas that are required to quantify the LOOHs and aldehydes. In the recorded single pulse experiment, the triglyceride peaks at 4.4 ppm need to be integrated. In the selective pulse experiment, the LOOH (11.3-10.6 ppm) and aldehyde area (9.9-9.2 ppm) need to be integrated (Figure A-2.1). The practical steps of the integration differ slightly per software-package, but the principle remains largely the same. Here, the integration steps for TopSpin are described. 


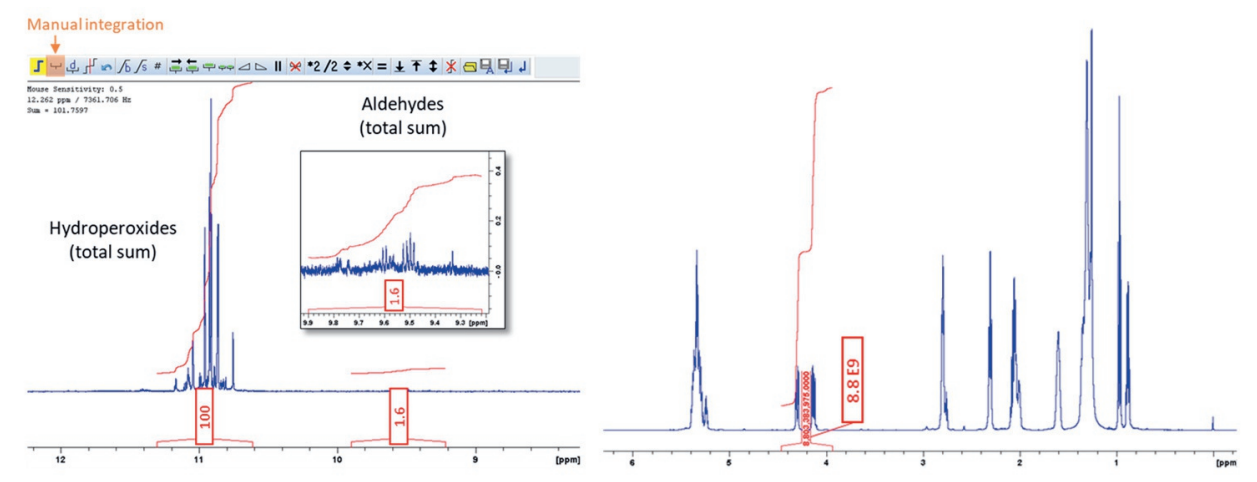

Figure A-2.1. Example of correct integration of the desired three regions. Left is the integration of the oxidation products in a selective pulse spectrum. Right is the integration of the triglyceride peak in the single pulse spectrum.

i. $\quad$ Open the integration module. [.INT]

ii. On the top left, make sure the small horizontal '-[' is toggled (If you hover over the correct button, it says 'Define new region using cursor. (toggle)')

iii. When integrating, include $0.1-0.2 \mathrm{ppm}$ of baseline on both sides of the peak of interest. This is important for the following step.

iv. To make sure the integral is not biased, make sure that both sides of the integral you take are horizontal (because integrating baseline should be zero). Do this by holding 'b' (b stands for bias and can be interpreted as baseline off-set) and dragging (or scrolling) it up or down to adjust the slope of the beginning of the integral. The same can be done for the end of the integral, using 's' (s stands for slope and can be interpreted as such). In Figure A-2.2, integrals with incorrect and correct 'b' and 's' values are shown.

v. Save and return when you are satisfied. (.sret)

vi. Optional:

a. If you want to save the integration settings and copy them to another spectrum: type 'wmisc intrng name', with a name for the integral. The saved integral settings can be found when typing 'rmisc' and can be used in different data-sets.

b. Then select another sample and type 'rmisc intrng name' to apply the same integral settings. When you use this option, it is recommended to go back to step 4 , and check the bias and slope of the integral. 
vii. Extract the absolute integral number, go to the tab 'integrals' and copy the number under 'integral [abs]'

a. Note that these absolute integral numbers are often large numbers that should be copied and pasted to a spreadsheet, rather than typed manually.

Note that this integration is describing the sum of hydroperoxides and the sum of aldehydes. Using the same approach, the individual lipid hydroperoxide and aldehydes can also be quantified. More details on the identification of these oxidation products can be found in Merkx et al. (2018).

A

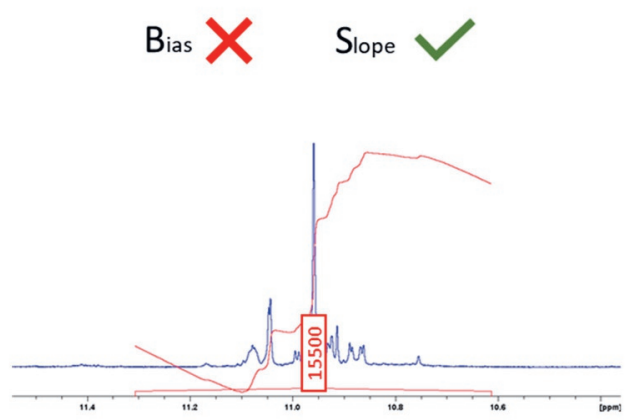

C

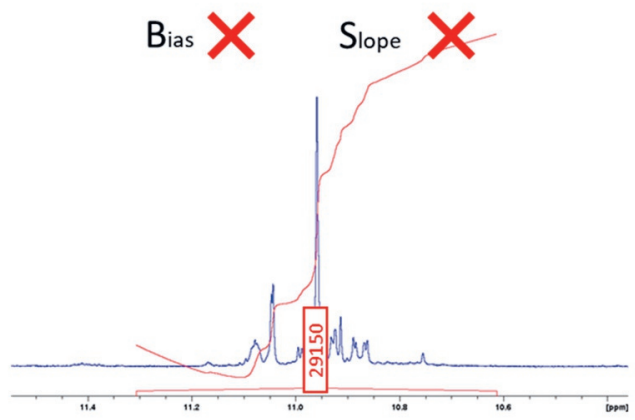

B

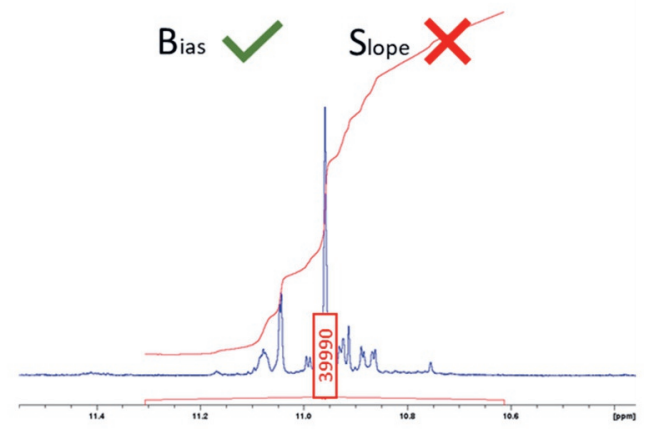

D

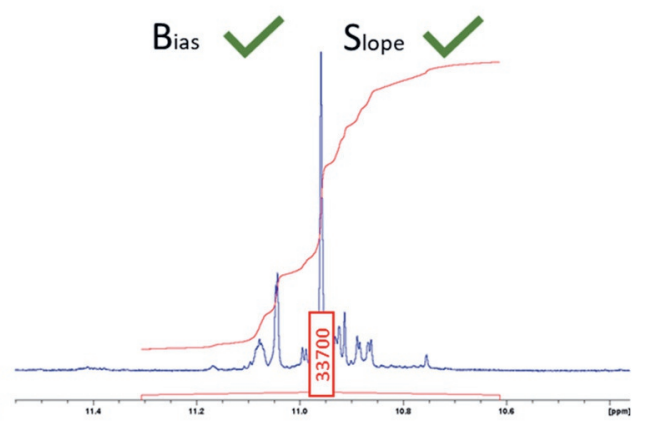

Figure A-2.2. The impact of Bias (b) and Slope (s) on spectral integration. Both sides of the integral should be horizontal. Modifying the Bias (b) and Slope (S) respectively impact the steepness of the beginning and end of the integral. Note the impact of the absolute integral numbers when the Bias and Slope are incorrect $(A, B, C)$ versus correct (D). 


\section{A-2.3.6. Calculation}

Traditionally, oxidation products are expressed as millimoles (mmol) or milliequivalents (meq) of oxidation product per kilogram oil. Therefore, the standard way this quantification method is set up yields the oxidation products in the ' $\mathrm{mmol} / \mathrm{kg}$ of oil' expression. The method does not require addition of an internal standard and instead uses the present triglyceride peak and its molar mass. The molar mass of the oil can be estimated easily by looking at the fatty acid composition of the used fat/oil. In the following calculation, a molar mass of $880 \mathrm{~g} / \mathrm{mol}$ is used.

The quantification can be done either by using only the single pulse NMR experiment or by using both the single pulse and the selective pulse experiments. One can use the first approach if the oxidation products are sufficiently present in the single pulse spectrum, which means that the $\mathrm{S} / \mathrm{N}$ ratio of the peaks should be above 10 . If this is not the case, the selective pulse is advised for accurate quantification.

Using only the single pulse experiment, the quantification can be performed as follows:

$$
\mathrm{c}_{\mathrm{ox}}(\mathrm{mmol} / \mathrm{kg})=\frac{\mathrm{I}_{\mathrm{ox}}}{\mathrm{I}_{\mathrm{TG}}} \cdot \frac{\mathrm{N}_{\mathrm{TG}}}{\mathrm{N}_{\mathrm{ox}}} \cdot \frac{10^{6}}{\mathrm{MW}_{\mathrm{TG}}}
$$

Here, $\mathrm{I}$ is the NMR signal intensity after integration, $\mathrm{N}$ is the number of protons and $\mathrm{MW}$ is the molecular weight of the fat or oil. Index ox stands for oxidized lipid product, TG for triglyceride. The integrals I need to be extracted from the spectrum as described under Integration/vii. The number of protons comes from prior knowledge of the system, so in case of LOOHs and aldehydes, $\mathrm{N}_{\mathrm{ox}}$ is 1 , whereas $\mathrm{N}_{\mathrm{TG}}$ is 4 . The $\mathrm{MW}_{\mathrm{TG}}$ needs to be estimated by the analyst as described before.

When the band selective pulses are used, several terms are added to the equation to correct for the use of different pulse sequences.

$$
\begin{aligned}
\mathrm{c}_{\mathrm{ox}}(\mathrm{mmol} / \mathrm{kg})= & \frac{\mathrm{I}_{\mathrm{ox}}}{\mathrm{I}_{\mathrm{TG}}} \cdot \frac{\mathrm{N}_{\mathrm{TG}}}{\mathrm{N}_{\mathrm{ox}}} \cdot \frac{\mathrm{ns}_{\mathrm{zg}}}{\mathrm{ns}_{\mathrm{sel}}} \cdot \frac{\sin \left(\mathrm{p}_{\mathrm{zg}}\right)}{\sin \left(\mathrm{p}_{\mathrm{sel}}\right)} \cdot \frac{\mathrm{RG}_{\mathrm{zg}}}{\mathrm{RG}_{\mathrm{sel}}} \cdot \frac{10^{6}}{\mathrm{MW}_{\mathrm{TG}}} \quad \text { Eq. A-2.2 } \\
& \cdot \text { SelFactor }
\end{aligned}
$$

Here, $n$ s is the number of scans, RG is the receiver gain, $\mathrm{p}$ is the pulse angle in degrees. Index $\mathrm{zg}$ stands for the direct pulse experiment and sel for the band-selective experiment. SelFactor stands for the empirically determined factor which corrects for the signal intensity loss due to relaxation differences between the single pulse and band-selective experiments. The SelFactor will typically have a value between 1.0 and 1.3 .

Note that the first time this quantification method is used for a new NMR system, the following steps should be performed to determine the SelFactor. 
i. Analyse one or more rancid oil (PV $>10 \mathrm{meq} / \mathrm{kg}$ ) according to the protocol. Record both single pulse as well as band selective pulse spectra.

a. Note that this step is to determine the SelFactor for your NMR system.

ii. Create a new spreadsheet file for the calculations.

iii. Retrieve the number of scans (NS) and receiver gain (RG) for both the single pulse and selective pulse experiments and put them in the spreadsheet as exemplified in Figure A-2.3.

a. If the single pulse is recorded with a pulse angle other than $90^{\circ}$, also include the sine of this angle in the spreadsheet, e.g., by (SIN(RADIANS(30)) in Microsoft Excel for the sinus of a $30^{\circ}$ degree pulse.

iv. Determine the Ox/TG corrected ratio for both spectra according to Eq A-2.3 (Eq A2.1 and Eq A-2.2 combined and reduced):

$$
\text { SelFactor }=\frac{I_{\mathrm{ox}}(\mathrm{zg})}{\mathrm{I}_{\mathrm{ox}}(\mathrm{sel})} \cdot \frac{\mathrm{ns}_{\mathrm{sel}}}{\mathrm{ns}_{\mathrm{zg}}} \cdot \frac{\mathrm{RG}_{\mathrm{sel}}}{\mathrm{RG}_{\mathrm{zg}}} \cdot \frac{\sin \left(\mathrm{p}_{\mathrm{sel}}\right)}{\sin \left(\mathrm{p}_{\mathrm{zg}}\right)}
$$

Single Pulse (ZG)

Band-Selective Pulse (SEL)

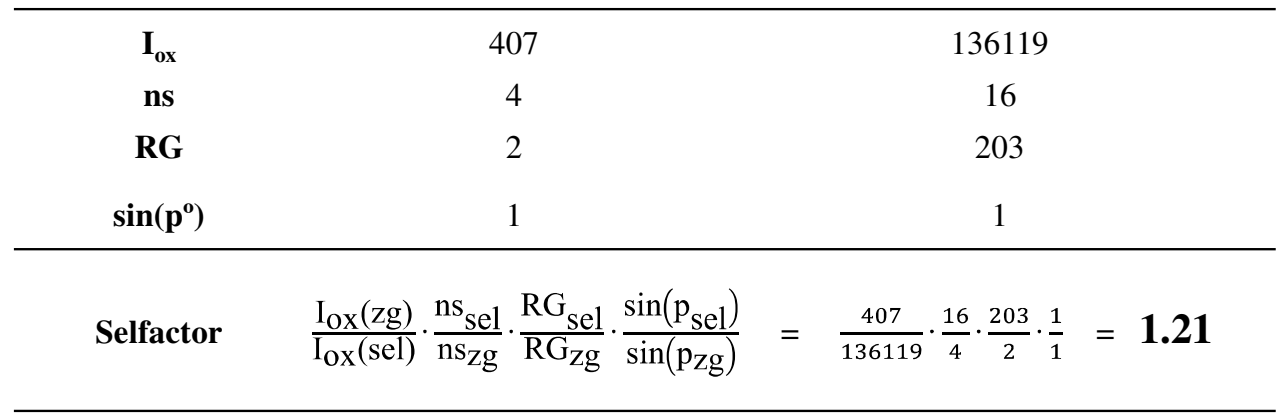

Figure A-2.3. Example on the calculation of the SelFactor, using both the single pulse (ZG) and band-selective pulse (SEL). Bases on the equation in 3.6.iv. 


\section{A-2.4. Notes}

I. The sensitivity of the method depends on the strength of the magnetic field, the sensitivity of the probe, and the number of scans used. Cryogenic probe heads can provide significant sensitivity enhancement, but are not commonly available. Increasing the magnetic field strength (i.e. using a $600 \mathrm{MHz}$ instead of a $400 \mathrm{MHz}$ ) is sometimes an option. The sensitivity (SNR) increase that is obtained from increasing the magnetic field strength $\left(\mathrm{B}_{0}\right)$ is: $\left(\frac{\mathrm{B}_{0}-\mathrm{High}}{\mathrm{B}_{0}-\mathrm{Low}}\right)^{1.5}$. The number of scans can always be increased, where increasing the number of scans by $\mathrm{x}$ will result in an increase in the SNR by the square root of x. For example, going from 16 to 256 scans will increase the SNR by a factor of $\sqrt{ }(256 / 16)=4$.

II. At temperatures higher than $290 \mathrm{~K}$, the samples in the NMR tube may partially phase separate after prolonged storage ( $>16$ hours). This will yield samples that are hard to shim and reduces the resolution of the NMR spectrum. To prevent this, either work with small series, manually tumble the NMR tubes every few hours, or use a cooled sample changer. The latter may however not be available in every facility. 


\section{A-2.5. Literature}

Antolovich, M., Prenzler, P. D., Patsalides, E., McDonald, S., \& Robards, K. (2002). Methods for testing antioxidant activity. Analyst, 127(1), 183-198.

Barriuso, B., Astiasarán, I., \& Ansorena, D. (2013). A review of analytical methods measuring lipid oxidation status in foods: a challenging task. European Food Research and Technology, 236(1), 1-15.

Dobarganes, M. C., \& Velasco, J. (2002). Analysis of lipid hydroperoxides. European Journal of Lipid Science and Technology, 104(7), 420-428.

Goicoechea, E., \& Guillen, M. D. (2010). Analysis of Hydroperoxides, Aldehydes and Epoxides by $1 \mathrm{H}$ Nuclear Magnetic Resonance in Sunflower Oil Oxidized at 70 and $100{ }^{\circ} \mathrm{C}$. Journal of Agricultural and Food Chemistry, 58(10), 6234-6245.

Guillen, M. D., \& Goicoechea, E. (2009). Oxidation of corn oil at room temperature: Primary and secondary oxidation products and determination of their concentration in the oil liquid matrix from $1 \mathrm{H}$ nuclear magnetic resonance data. Food Chemistry, 116(1), 183-192.

Guillén, M. D., \& Ruiz, A. (2008). Monitoring of heat-induced degradation of edible oils by proton NMR. European Journal of Lipid Science and Technology, 110(1), 52-60.

Merkx, D. W. H., Hong, S., Ermacora, A., \& van Duynhoven, J. (2018). Rapid Quantitative Profiling of Lipid Oxidation Products in a Food Emulsion by 1H NMR. Analytical Chemistry.

Schaich, K. M. (2020). Lipid Oxidation: New Perspectives on an Old Reaction. In F. Shahidi (Ed.), Bailey's Industrial Oil and Fat Products, (7th ed.): John Wiley \& Sons, Ltd.

Skiera, C., Steliopoulos, P., Kuballa, T., Holzgrabe, U., \& Diehl, B. (2012a). 1H-NMR Spectroscopy as a New Tool in the Assessment of the Oxidative State in Edible Oils. Journal of the American Oil Chemists' Society, 89, 1383-1391.

Skiera, C., Steliopoulos, P., Kuballa, T., Holzgrabe, U., \& Diehl, B. (2012b). 1H NMR approach as an alternative to the classical p-anisidine value method. European Food Research and Technology, 235, 1101-1105. 



$$
3
$$




\section{Chapter 3}

\section{Lipid epoxide quantification in a food emulsion by ${ }^{1} \mathrm{H}-{ }^{13} \mathrm{C}$ HSQC NMR spectroscopy}

Based on: Merkx, D. W. H. ${ }^{1}$, Boerkamp, V. J. P. ${ }^{1}$, Wang, J., Hennebelle, M., \& van Duynhoven, J. P. M. Lipid epoxide quantification in a food emulsion by ${ }^{1} \mathrm{H}-{ }^{13} \mathrm{C}$ HSQC NMR spectroscopy, to be submitted 


\begin{abstract}
Lipid oxidation is a chemical process that is detrimental for the quality of oil-based foods. Historically, hydroperoxides (LOOHs) and aldehydes are considered the most important molecules in the assessment of lipid oxidation. While they do provide insights in kinetics of primary and secondary oxidation, it has been proposed that a third class of oxidation products, the epoxides, could help resolve mechanistical anomalies that are hitherto unexplained. Here, we developed a $2 \mathrm{D}{ }^{1} \mathrm{H}_{-}{ }^{13} \mathrm{C}$ HSQC NMR method that allows for a quantitative assessment of epoxides in real food products. The method was applicable in vegetable oils and mayonnaises in a reproducible (11.6\% RSD) and repeatable (8.2\% RSD) manner with an LoD and LoQ of respectively 0.18 and $0.62 \mathrm{mmol} / \mathrm{kg}$.

Finally, the method was applied in combination with other NMR methods to assess LOOHs and aldehydes to provide a comprehensive view on lipid oxidation pathways under different conditions. Here, we varied sample matrix and storage temperature. We found that epoxides have a considerable contribution to the overall oxidation product range that is often overlooked. Its potential as a sole early marker for lipid oxidation, however, appeared to be limited. Assessing epoxides, in combination with hydroperoxides and aldehydes, can be a powerful tool to provide a more comprehensive mechanistical understanding on lipid oxidation pathways. Moreover, the developed HSQC-method allowed for characterization of individual epoxide sub-classes, enabling mechanistical oxidation studies.
\end{abstract}




\subsection{Introduction}

Lipid oxidation is a concern for the shelf-life of many food products that are high in unsaturated fatty acids, such as oils and dressings. It is linked to the formation of off-flavours and off-odours and, in later oxidation stages, can even physically destabilise the food product. Lipid oxidation is classically described as a free radical chain reaction that can be divided in primary and secondary oxidation. In primary oxidation, a (bis)allylic hydrogen is abstracted, forming a radical that can react with $\mathrm{O}_{2}$ to yield a peroxyl radical. This peroxyl radical can propagate the free radical chain reaction by abstracting a hydrogen from another fatty acid, forming hydroperoxides (LOOHs) in the process. Secondary oxidation is the degradation of these hydroperoxides, where one of the main products that are formed are the aldehydes, which are also potent off-flavours. This binary comprehension of the lipid oxidation mechanism has suited the industry and academia for a long period, but, in recent years, there has been an interest in more detailed assessment of the chemical mechanisms of lipid oxidation.

A major class that has been often overlooked are the epoxides (Schaich, Xie, \& Bogusz, 2017), which can be formed from peroxyl radicals during primary oxidation, or via alkoxyl radicals, the reactants of classical secondary oxidation. These epoxide generating mechanisms dependent on environmental conditions such as temperature, oxygen availability, and protic solvent availability (Schaich et al., 2017). Furthermore, there are reports that epoxides might be a potential novel candidate as early marker of lipid oxidation in food (Grüneis \& Pignitter, 2018). Currently, due to lack of rapid and quantitative methods, epoxides are rarely analysed in lipid oxidation studies, which makes their actual impact and importance is still poorly understood. The official AOCS method (Cd 9-57) to quantify epoxides is a hydrogen bromide $(\mathrm{HBr})$ titration with a low sensitivity and is influenced by the presence of conjugated dienes (Xia, Budge, \& Lumsden, 2015) and molecular oxygen (Liao, 2013). More recently, a novel LC-MS method has been developed to detect epoxides and hydroperoxides simultaneously, unfortunately with a time consuming pre-concentration step that hampered the throughput (Grüneis et al., 2019). So far, a major impediment for unravelling the mechanistic role of epoxides and validating their use as early marker is the lack of simple and high-throughput quantitative methods.

Nuclear Magnetic Resonance (NMR) has been shown in recent years as a convenient tool to assess lipid oxidation products quantitatively and rapidly (Merkx, Hong, Ermacora, \& van Duynhoven, 2018; Skiera, Steliopoulos, Kuballa, Holzgrabe, \& Diehl, 2012). NMR has been used to quantify epoxides in chemically and thermally epoxidised fatty acid esters and oil samples, when most double bonds are converted into epoxides (Goicoechea \& Guillen, 2010; Xia et al., 2015; Xia \& Budge, 2017; Xia, Budge, \& Lumsden, 2016). When epoxides are 
obtained via auto-oxidation under moderate shelf-life conditions, the epoxide structures are more diverse, and their concentrations are orders of magnitude lower. This results in 1D spectra with significant overlap of the epoxide and unreacted bisallylic protons which severely compromises quantification. A recourse for overcoming overlap in 1D NMR is the use of 2D NMR (Van Duynhoven, 2013). Here, we propose the use of 2D Heteronuclear Single Quantum Coherence (HSQC) spectroscopy to separate the epoxy signals from the bisallylic signals. Whereas the signal area in $1 \mathrm{D}$ NMR spectra is solely dependent on the number of equivalent spins, this is not the case in HSQC. Here, due to differences in longitudinal/transversal relaxation times and homo-/heteronuclear coupling constants, the signal intensity is not inherently quantitative (Fardus-Reid, Warren, \& Le Gresley, 2016; Giraudeau, 2014). To obtain quantitative results, one can modify the acquisition parameters to correct for heteronuclear coupling constants (Heikkinen, Toikka, Karhunen, \& Kilpeläinen, 2003), determine a correction factor theoretically (Peterson \& Loening, 2007), or determine a response factor empirically using samples with known analyte concentrations (Lewis et al., 2007). This last option will be further explored in this work.

In this paper, we describe the development of a novel 2D HSQC NMR method for the quantification of epoxides in real food systems, particularly rapeseed oil and mayonnaise. First, the method development and validation will be discussed. This 2D HSQC method will then be combined with other qNMR techniques to comprehensively assess the formation of hydroperoxides, aldehydes, and epoxides under accelerated shelf-life conditions. Here, we will vary temperature $\left(40{ }^{\circ} \mathrm{C}\right.$ vs $60^{\circ} \mathrm{C}$ ) and sample matrix (rapeseed oil vs. mayonnaise) to evaluate the significance of epoxide generation as an early marker and discuss further mechanistic implications.

\subsection{Material and Methods}

\subsubsection{Materials}

$\mathrm{CDCl}_{3}$ with $0.03 \%$ tetramethylsilane (TMS), DMSO- $d_{6}$ and deuterated $4 \AA$ molsieves were purchased from Euriso-top (Saint-Aubin, France). Rapeseed (RP) oil, egg yolk, sodium chloride, and spirit vinegar were purchased from local suppliers. Formic acid, hydrogen peroxide, and ethyl acetate were purchased from Sigma Aldrich (Zwijndrecht, the Netherlands). 


\subsubsection{Chemical epoxidation of rapeseed oil}

To compare the 1D ${ }^{1} \mathrm{H}$ NMR and 2D ${ }^{1} \mathrm{H}-{ }^{13} \mathrm{C}$ HSQC spectra of epoxidised samples without bisallylic protons, rapeseed oil was chemically epoxidised using formic acid and hydrogen peroxide, as described by Xia et al. (2016). In brief, formic acid and hydrogen peroxide were added to fresh rapeseed oil in a molar ratio of 1:3:2 (formic acid:hydrogen peroxide:double bonds). The mixture was incubated under head-over-tail rotation for $16 \mathrm{~h}$ at $38{ }^{\circ} \mathrm{C}$. Afterwards, the sample was mixed with $15 \mathrm{~mL}$ ethyl acetate and $22.5 \mathrm{~mL}$ brine and subsequently centrifuged $\left(4700 \mathrm{x} g, 5 \mathrm{~min}, 20^{\circ} \mathrm{C}\right)$. The epoxidised oil was recovered by drying the top layer under nitrogen flow.

\subsubsection{Mayonnaise preparation}

Mayonnaises with $78 \%$ w/w oil were prepared by emulsification with a Silverson mixer (Silverson, USA). Egg yolk (5\% w/w), $\mathrm{NaCl}(1.1 \% \mathrm{w} / \mathrm{w})$ were mixed with demineralised water to obtain the aqueous phase (in total $20.6 \% \mathrm{w} / \mathrm{w})$. Next, rapeseed oil $(78 \% \mathrm{w} / \mathrm{w}$ ) was slowly added to form the emulsion, and finally spirit vinegar $(1.4 \% \mathrm{w} / \mathrm{w})$ was added to achieve the desired $\mathrm{pH}$ (3.8).

\subsubsection{Accelerated shelf-life experiments}

For both the RP oils and the mayonnaises, aliquots of $1 \mathrm{~mL}$ were stored in $20.4 \mathrm{~mL}$ clear headspace vials with screwcaps at 40 and $60^{\circ} \mathrm{C}$. Sample frequency and storage duration were based on the oxidation level which depended on the sample matrix and storage conditions. Samples stored at $40{ }^{\circ} \mathrm{C}$ were sampled one to three times per week for a total duration of twelve weeks and the samples at $60{ }^{\circ} \mathrm{C}$ were sampled four to seven times per week for a total duration of three weeks. All samples were stored at $-20^{\circ} \mathrm{C}$ for at least 48 hours prior to analysis. The oxygen level was monitored with a MOCON OpTech- $\mathrm{O}_{2}$ oxygen sensor (Ametek Mocon, USA). The theoretical amount of oxygen that was used was calculated by assuming a $19.4 \mathrm{~mL}$ headspace with $\mathrm{O}_{2}$-max as measured by the oxygen sensor after stabilization and $46.8 \mathrm{mg} / \mathrm{kg}$ oxygen concentration in the oil (Cuvelier, Soto, Courtois, Broyart, \& Bonazzi, 2017). For each sample matrix and storage temperature, five samples were stored, and monitored over time. 


\subsubsection{NMR sample preparation}

Chemically epoxidised and autoxidised rapeseed oil were used without modification. Mayonnaise samples were first freeze-thawed to break the emulsion and to yield a clean oil layer after centrifugation ( $15 \mathrm{~min} ; 22,000 \mathrm{x} g ; 2{ }^{\circ} \mathrm{C}$ ). For both RP oil and the oil layer of mayonnaise, $150 \mu \mathrm{L}$ was dissolved in $450 \mu \mathrm{L} \mathrm{CDCl}_{3}$ (method development) or 5:1 $\mathrm{CDCl}_{3}:$ DMSO- $d_{6}$ (method development and shelf-life study) and transferred to 5-mm NMR tubes.

\subsubsection{NMR experimental setup}

All NMR spectra were recorded on a $600 \mathrm{MHz}(14.1 \mathrm{~T})$ Bruker Avance III NMR spectrometer (Bruker BioSpin, Switzerland) equipped with a cryo-probe. The internal temperature of the probe was set at $295 \mathrm{~K}$.

\subsubsection{Epoxy signal assignment}

For the epoxy signal assignment, a rapeseed oil was used that was stored for sixteen days under unlimited oxygen atmosphere at $60{ }^{\circ} \mathrm{C}$. A $2 \mathrm{D}{ }^{1} \mathrm{H}^{13} \mathrm{C}$ HSQC spectrum was recorded using the standard "hsqcetgpsisp2.2" Bruker pulse sequence. In the ${ }^{13} \mathrm{C}$-dimension, the spectral width was $200 \mathrm{ppm}$ with an off-set of $\delta 72.8 \mathrm{ppm}\left(\delta_{\mathrm{C}} 172.8\right.$ to $\left.-27.2 \mathrm{ppm}\right)$ and with 4096 increments. In the ${ }^{1} \mathrm{H}$-dimension, the spectral width was $16 \mathrm{ppm}$ with an off-set of 5 ppm $\left(\delta_{\mathrm{H}} 5\right.$ to $\left.1 \mathrm{ppm}\right)$ with 8192 increments. Sixteen scans were collected using a relaxation time of $1.5 \mathrm{~s}$, an automatically determined $90^{\circ}$ pulse length, and the INEPT delay tuned to an assumed ${ }^{1} J_{\mathrm{CH}}$ of $170 \mathrm{~Hz}$. The standard "hsqcetgpml" Bruker pulse sequence was used to record 2D TOCSY-HSQC spectra. Three spectra were recorded which differed in mixing time (20,50, and $80 \mathrm{~ms})$, using the settings above, except for the number of increments which was set to 2048 and 4096 for the ${ }^{13} \mathrm{C}$ and ${ }^{1} \mathrm{H}$-dimension, respectively. For the Fourier transformation in the ${ }^{1} \mathrm{H}$-dimension of the HSQC experiment, Gaussian apodization was used with a Gaussian max position of 0.001 . For the ${ }^{13} \mathrm{C}$-dimension, zero-filling up to 1024 points was applied prior to Fourier transformation with a squared cosine window function. For processing the TOCSY-HSQC experiments, both dimensions were filled up to 4096 points prior to a Fourier transform using a qsine window function and SSB of 2. For all spectra, phase correction was performed manually, baseline correction was performed automatically, and TMS was calibrated to $\delta_{\mathrm{H}} 0 \mathrm{ppm}$ and $\delta_{\mathrm{C}} 0 \mathrm{ppm}$. 


\subsubsection{Quantification of epoxides}

Unless mentioned otherwise, the same settings as described in previous section were used in the $2 \mathrm{D}{ }^{1} \mathrm{H}-{ }^{13} \mathrm{C}$ HSQC-experiments for epoxide quantification. Spectra were recorded using a standard "hsqcetgpsisp2.2" Bruker pulse sequence. In the ${ }^{13} \mathrm{C}$-dimension, the spectral width was $200 \mathrm{ppm}$ with an off-set of $\delta 72.8 \mathrm{ppm}\left(\delta_{\mathrm{C}} 172.8\right.$ to $\left.-27.2 \mathrm{ppm}\right)$ and with 400 increments. In the ${ }^{1} \mathrm{H}$-dimension, the spectral width was $4 \mathrm{ppm}$ with an off-set of $3 \mathrm{ppm}\left(\delta_{\mathrm{H}} 5\right.$ to $\left.1 \mathrm{ppm}\right)$ with 2048 increments. Twelve scans were collected using a relaxation time of $0.8 \mathrm{~s}$. Spectral processing was done as described in the previous section. The upfield triglycerol (TG) backbone peak was calibrated to $\delta_{\mathrm{H}} 4.13 \mathrm{ppm}$ and $\delta_{\mathrm{C}} 61.9 \mathrm{ppm}$. Integration was done automatically using predefined elliptical integration ranges in MestReNova v14.1 (Mestrelab Research, S.L., Santiago de Compostela, Spain). Details on the integrations can be found in Table S3.1. The concentration of epoxides was expressed in $\mathrm{mmol} / \mathrm{kg}$ oil and calculated using Equation 3.1 for the 1D experiment (for the chemically epoxidized oil samples) and Equation 3.2 for the 2D HSQC experiment.

$$
\begin{gathered}
\mathrm{c}_{\text {epox }}(\mathrm{mmol} / \mathrm{kg})=\frac{\mathrm{I}_{\text {epox }}^{1 \mathrm{D}}}{\mathrm{I}_{\mathrm{TG}}^{1 \mathrm{D}}} \cdot \frac{\mathrm{N}_{\mathrm{TG}}}{\mathrm{N}_{\text {epox }}} \cdot \frac{10^{6}}{\mathrm{MW}_{\mathrm{TG}}} \\
\mathrm{c}_{\text {epox }}(\mathrm{mmol} / \mathrm{kg})=\frac{\mathrm{I}_{\text {epox }}^{2 \mathrm{D}}}{\mathrm{I}_{\mathrm{TG}}^{2 \mathrm{D}}} \cdot \frac{\mathrm{N}_{\mathrm{TG}}}{\mathrm{N}_{\text {epox }}} \cdot \frac{10^{6}}{\mathrm{MW}_{\mathrm{TG}}} \cdot \mathrm{K}
\end{gathered}
$$

Here, I was the NMR signal integral, $\mathrm{N}$ the number of protons, and MW the molecular weight (average triglyceride $\sim 880 \mathrm{~g} \cdot \mathrm{mol}^{-1}$ ). Index 'epox' stands for epoxide, 'TG' for triglyceride. The factor $\mathrm{K}$ was the empirically determined factor that links the $1 \mathrm{D}$ response to the $2 \mathrm{D}$ response of both TG and epoxy moieties, as described in Eq 4-7. The spectral integration region to determine $\mathrm{I}_{\mathrm{TG}}$ was $\delta_{\mathrm{H}} 4.4$ to $4.0 \mathrm{ppm}$, with $\mathrm{N}_{\mathrm{TG}}$ corresponding to the four outer protons of the TG backbone. The TG backbone was not expected to undergo changes upon oxidation, hence its ${ }^{1} \mathrm{H}$ NMR signal was used as internal standard (Skiera et al., 2012). The integrals $\mathrm{I}_{\mathrm{epox}}$ are determined from the spectral integration regions listed in Table 1, all with $\mathrm{N}_{\text {epox }}$ corresponding to 2 . The proposed correction factor $\mathrm{K}$ was built on the theoretical constant $\mathrm{k}$ (Lewis et al., 2007), according to Eq 3.3. Combining Eq. 3.3 with Eq. 3.1 and Eq. 3.2 yielded the Equations 3.4-3.7. The assumption was made that the concentration (c) is proportional to the $1 \mathrm{D}$-integral (I) divided by the number of protons $(\mathrm{N})$. 


$$
\begin{gathered}
\mathrm{V} \text { or } \mathrm{I}^{2 \mathrm{D}}=\mathrm{k} \cdot \mathrm{c} \cdot \mathrm{N} \cdot \mathrm{Vs} \\
\frac{\mathrm{I}_{\text {epox }}^{2 \mathrm{D}}}{\mathrm{I}_{\mathrm{TG}}^{2 \mathrm{D}}}=\frac{\mathrm{k}_{\text {epox }}}{\mathrm{k}_{\mathrm{TG}}} \cdot \frac{\mathrm{c}_{\text {epox }}}{\mathrm{C}_{\mathrm{TG}}} \cdot \frac{\mathrm{N}_{\mathrm{epox}}}{\mathrm{N}_{\mathrm{TG}}} \cdot \frac{\mathrm{Vs}}{\mathrm{Vs}} \\
\frac{\mathrm{I}_{\text {epox }}^{2 \mathrm{D}}}{\mathrm{I}_{\mathrm{TG}}^{2 \mathrm{D}}}=\frac{\mathrm{k}_{\text {epox }}}{\mathrm{k}_{\mathrm{TG}}} \cdot \frac{\mathrm{I}_{\text {epox }}^{1 \mathrm{D}}}{\mathrm{I}_{\mathrm{TG}}^{1 \mathrm{D}}} \cdot \frac{\mathrm{N}_{\mathrm{TG}}}{\mathrm{N}_{\mathrm{epox}}} \cdot \frac{\mathrm{N}_{\mathrm{epox}}}{\mathrm{N}_{\mathrm{TG}}} \\
\frac{\mathrm{I}_{\mathrm{epox}}^{2 \mathrm{D}}}{\mathrm{I}_{\mathrm{TG}}^{2 \mathrm{D}}}=\mathrm{K} \cdot \frac{\mathrm{I}_{\text {epox }}^{1 \mathrm{D}}}{\mathrm{I}_{\mathrm{TG}}^{1 \mathrm{D}}}, \text { where } \mathrm{K}=\frac{\mathrm{k}_{\mathrm{epox}}}{\mathrm{k}_{\mathrm{TG}}} \\
\mathrm{K}=\frac{\mathrm{I}_{\text {epox }}^{1 \mathrm{D}}}{\mathrm{I}_{\mathrm{TG}}^{1 \mathrm{D}}} \cdot \frac{\mathrm{I}_{\mathrm{TG}}^{2 \mathrm{D}}}{\mathrm{I}_{\mathrm{epox}}^{2 \mathrm{D}}}
\end{gathered}
$$

Here, $\mathrm{V}$ or $\mathrm{I}^{2 \mathrm{D}}$ was the peak volume or $2 \mathrm{D}$-integral, $\mathrm{k}$ is the HSQC-constant, $\mathrm{c}$ the concentration, $\mathrm{N}$ the number of protons, Vs the sensitive coil volume and $\mathrm{K}$ the combined epoxide-constant. The main requirement for the determination of $\mathrm{K}$ is that the epoxide and TG region can be accurately integrated in both 1D and 2D (HSQC) spectra. A K-factor of 1.16 was used in this research.

\subsubsection{Quantification of lipid hydroperoxides and aldehydes}

Hydroperoxides and aldehydes were quantified using a single pulse and band selective ${ }^{1} \mathrm{H}$ NMR spectra. Details on the assignment of signals and quantification can be found in Merkx et al. (2018).

\subsubsection{Validation of epoxide method}

The repeatability (r) and within-laboratory reproducibility $(\mathrm{R})$ were determined by measuring three auto-oxidised oil samples in duplicate for four days (total 24 samples). Three epoxide concentrations were assessed to mimic anticipated levels during real storage. The $\mathrm{r}$ and $\mathrm{R}$ for each concentration level were obtained using an ANalysis Of VAriance (ANOVA) model. Results were expressed as relative standard deviations of repeatability $\left(\mathrm{RSD}_{\mathrm{r}}\right)$ and withinlaboratory reproducibility $\left(\mathrm{RSD}_{\mathrm{R}}\right)$. The limit of detection $(\mathrm{LoD})$ and limit of quantification (LoQ) were respectively based on estimates of three and ten times the standard deviation from ten samples with an epoxide concentration lower than $1 \mathrm{mmol} / \mathrm{kg}$. The trueness of epoxide quantification using ${ }^{1} \mathrm{H}$ NMR was previously shown by Xia et al. (2015). 


\subsubsection{Sigmoidal model fitting}

Experimental data on the shelf-life studies on all oxidation products were fitted with a sigmoidal model developed by Gompertz (1825), with the addition of an off-set constant, according to Eq. 3.8. Only data points up until a secondary acceleration or degradation phase were used in the regression analysis.

$$
\mathrm{c}_{\mathrm{ox}}=\mathrm{A} \cdot \mathrm{e}^{-\mathrm{e}^{-\mathrm{k}\left(\mathrm{t}-\mathrm{t}_{0}\right)}+\mathrm{c}_{\mathrm{ox}, \mathrm{t}_{0}}}
$$

where $\mathrm{c}_{\mathrm{ox}}$ is the concentration of an oxidation product class $(\mathrm{mmol} / \mathrm{kg}), \mathrm{A}$ is the upper asymptote value $(\mathrm{mmol} / \mathrm{kg}), \mathrm{k}$ is the oxidation rate constant, and $\mathrm{c}_{\mathrm{ox}, \mathrm{t}_{0}}$ is the initial concentration of the oxidation product $(\mathrm{mmol} / \mathrm{kg}) . \mathrm{c}_{\mathrm{ox}, \mathrm{t}_{0}}$ was kept constant for within every oxidation class at 0,2 , and $0.3 \mathrm{mmol} / \mathrm{kg}$ for the hydroperoxides, epoxides, and aldehydes, respectively.

\subsection{Results and Discussion}

\subsubsection{Heteronuclear NMR spectral assignments of epoxy signals}

To explore the scope of NMR for the quantification of epoxides in mildly oxidised food, fresh rapeseed (RP) oil (Figure 3.1A/D/G) was both chemically epoxidised (Figure 3.1B/E) and thermally oxidised (Figure 3.1C/F/H). In chemically epoxidised RP oil, the epoxy signals $\left(\delta_{\mathrm{H}} 3.20\right.$ to $2.85 \mathrm{ppm}$ ) were baseline separated in the 1D spectrum, similar to reported by Xia et al. (2015). Here, the absence of bisallylic signals ( $\delta_{\mathrm{H}} 2.85$ to $2.70 \mathrm{ppm}$ ) showed that the double bonds were fully converted to the epoxy moieties. This observation was in stark contrast to thermally oxidised RP oil (Figure 3.1C/F/H), where the bisallylic signals decreased only by $45 \%$ compared to the fresh RP oil. When zooming in at the epoxy region for the thermally oxidised sample, it appeared that epoxides were indeed accumulating. Quantification of these epoxides was impossible using the 1D spectrum, due to the high amount of overlapping bisallylic proton. Therefore, we investigated the possibility of using 2D HSQC to capitalise on the added resolution in the carbon dimension. 

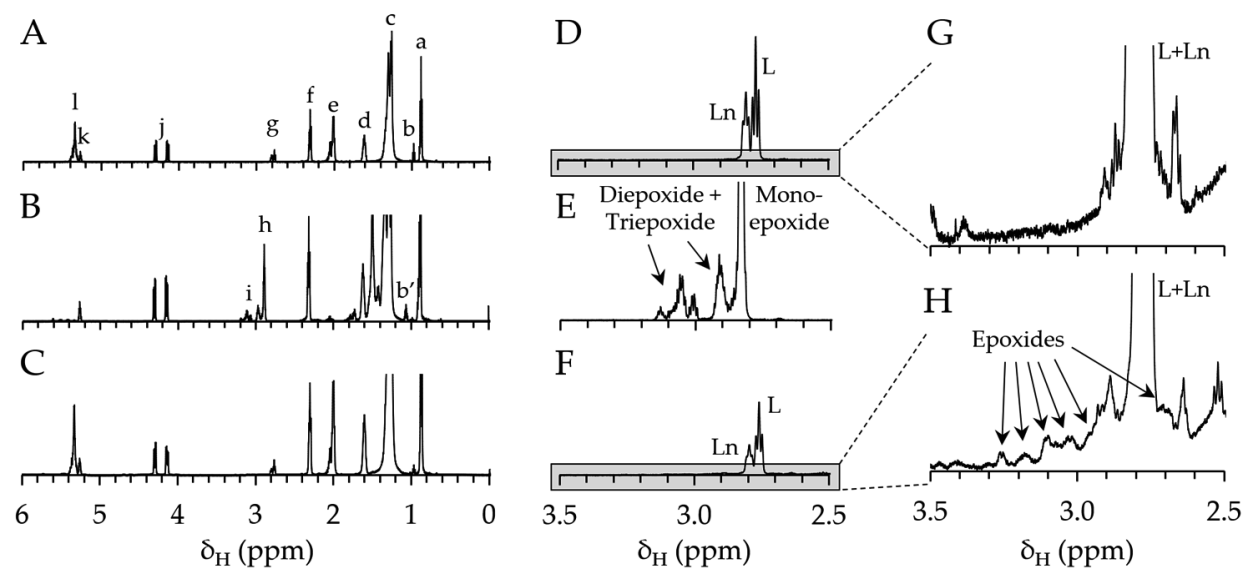

Figure 3.1. ${ }^{1} \mathrm{H}$ NMR spectra of (A, D) non-oxidised rapeseed (RP) oil, (B, E) chemically epoxidised and (C, F) thermally (ep)oxidised RP oil ([LOOH] $=200 \mathrm{mmol} / \mathrm{kg})$. In $(\mathrm{G})$ and $(\mathrm{H})$ zoomed in ${ }^{1} \mathrm{H}$ NMR spectra are shown of the epoxy region, large tails of the bisallylic protons from linoleic acid (L) and linolenic acid (Ln) are observed. All spectra were recorded in $\mathrm{CDCl}_{3}$ at $600 \mathrm{MHz}$. Spectral assignments can be found in Table S3.1.

In the 2D HSQC spectrum of the chemically epoxidised oil (Figure $3.2 \mathrm{~A}$ ), the epoxy ${ }^{13} \mathrm{C}-{ }^{-1} \mathrm{H}$ crosspeaks again laid well separated and isolated from the bisallylic signal at $\delta_{\mathrm{C}} 25.5 \mathrm{ppm}$, allowing for both assignment and quantification of the peaks. The triglyceride backbone $\mathrm{CH}_{2-}$ peaks (TG) were also well separated and relatively close to the epoxy signals. Therefore, the spectral region for setting up a quantitative method included both the TG and epoxy signals ( $\delta_{\mathrm{H}} 4.4$ to $2.5 \mathrm{ppm} ; \delta_{\mathrm{C}} 70$ to $40 \mathrm{ppm}$ ). Since chemically epoxidised rapeseed oil contained different epoxy moieties than thermally oxidised RP oil, the promise of using HSQC for the quantification of epoxides needed further confirmation in thermally oxidised sample as well. In these samples, the epoxy signals were again adequately separated from neighbouring peaks (Figure 3.2C) and the bisallylic signals. This allowed us to further explore the possibilities of using HSQC as a quantitative tool for the assessment of epoxides. 
A

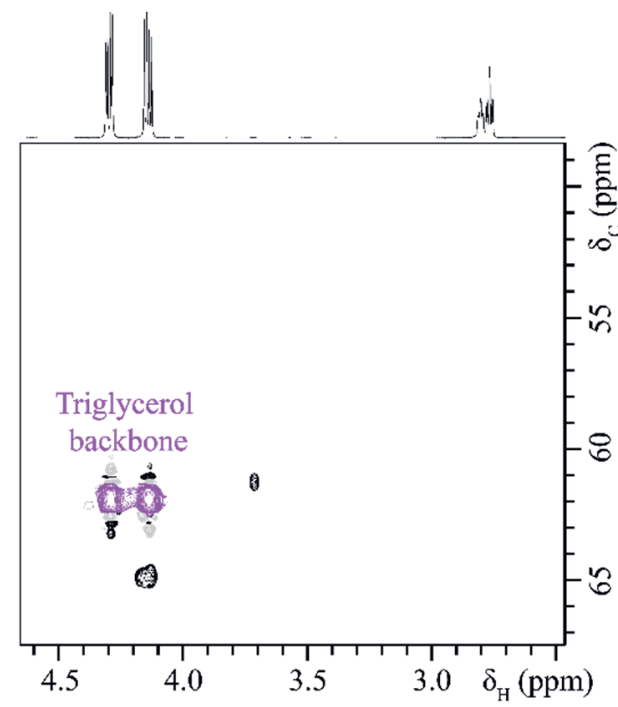

$\mathrm{C}$

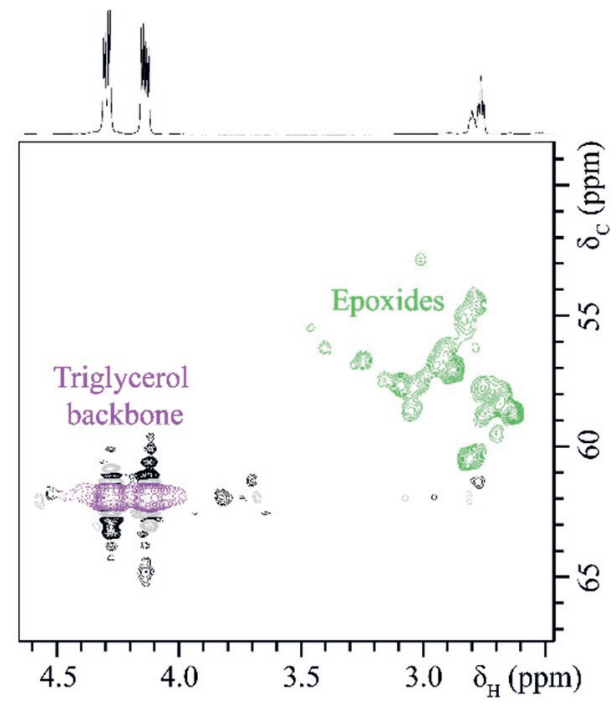

B

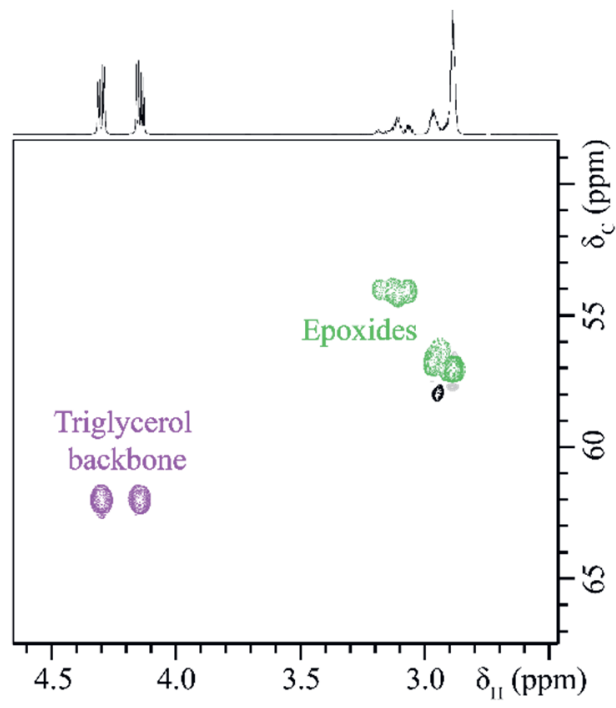

Figure 3.2. $2 \mathrm{D}$ HSQC NMR spectra $(600 \mathrm{MHz})$ of a non-oxidised rapeseed (RP) oil (A), chemically epoxidised RP oil (B), and a thermally (ep)oxidised RP oil $\left(15 \mathrm{~d}\right.$ at $\left.60^{\circ} \mathrm{C}\right)(\mathrm{C})$. In the $\mathrm{F} 1$ and $\mathrm{F} 2$ direction, the chemical shifts of respectively ${ }^{13} \mathrm{C}$ and ${ }^{1} \mathrm{H}$ are plotted. Note that contours in $\mathrm{B}$ were plotted at a higher level compared to $\mathrm{A}$ and $\mathrm{C}$ as epoxide concentrations were orders of magnitude higher.

First, the advantage of separating signals in the ${ }^{13} \mathrm{C}$ dimension of the HSQC was exploited to assign the signals of structural elements containing epoxy moieties (Figure 3.3, Table 3.1,). This assignment was done on four of the main epoxides in thermally oxidised rapeseed oil, using 2D TOCSY-HSQC experiments (details in Figure S3.2). Since RP oil is rich in oleic acid (18:1) and linoleic acid (18:2), we could distinguish between cis- and trans-isomers of 
generic epoxides and the cis/trans-epoxides that have a conjugated hydroperoxide-diene system at the $\alpha$-position of the epoxides. We refrained from assigning remaining minor peaks.

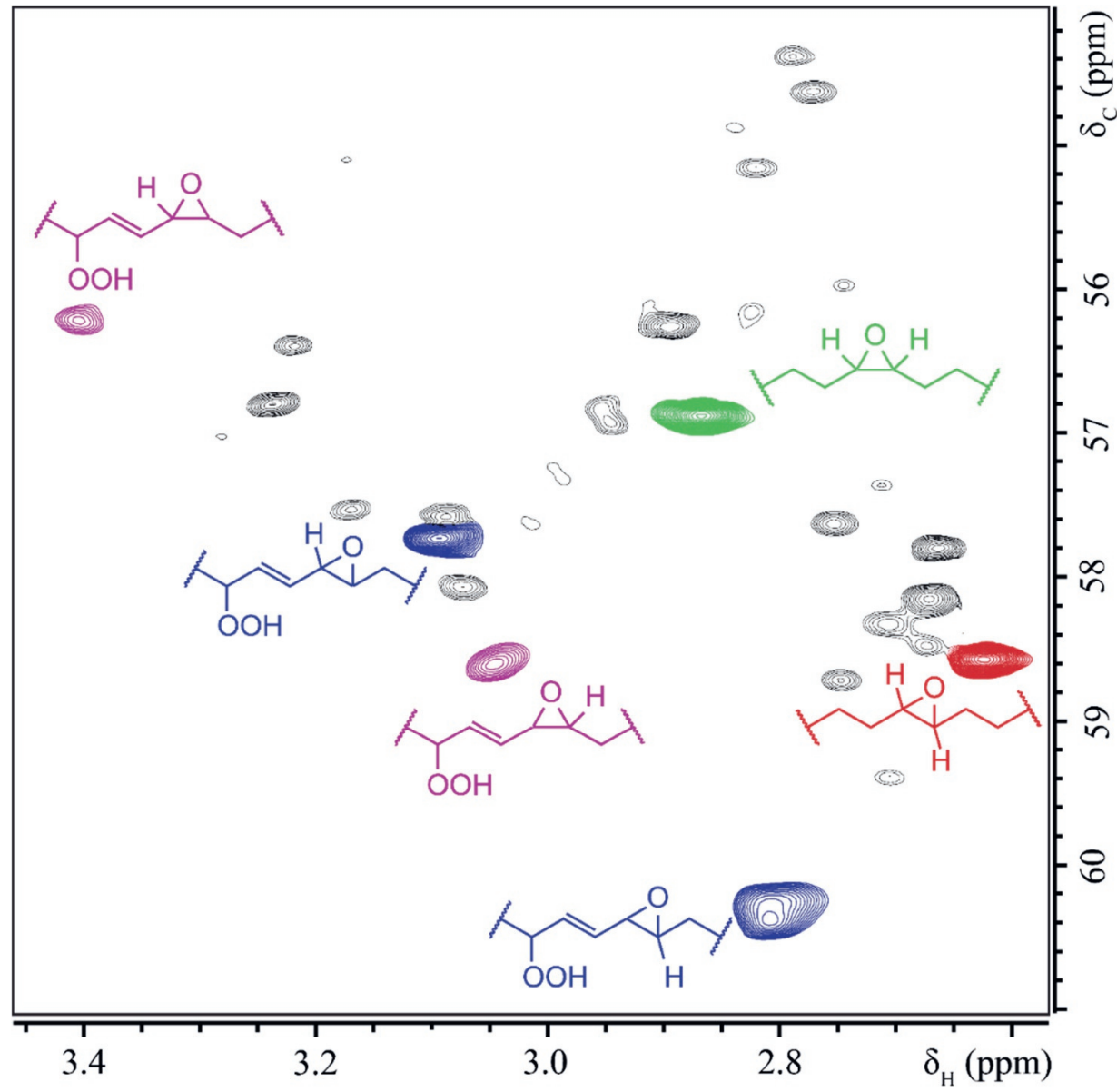

Figure 3.3. 2D HSQC NMR spectrum of thermally epoxidised RP oil with the annotation of the most abundant peaks. The structures that are drawn next to the peaks are snapshots of the functional groups, where $\mathrm{CH}_{2}$-groups can be interchanged with olefines depending on the parent molecule. 
Table 3.1 Annotation of epoxy signals in $\mathrm{CDCl}_{3}: \mathrm{DMSO}_{-} \mathrm{d}_{6}(5: 1)$, as shown in Figure 3.3. TMS was used as reference compound ( $\left.\delta_{\mathrm{C}} 0 \mathrm{ppm} ; \delta_{\mathrm{H}} 0 \mathrm{ppm}\right)$.

\begin{tabular}{|c|c|c|c|}
\hline Trivial name & Structural formula & $\delta_{\mathrm{C}}(\mathrm{ppm})$ & $\delta_{\mathrm{H}}(\mathrm{ppm})$ \\
\hline Trans-Epox-oleate & & 58.6 & 2.62 \\
\hline Cis-Epox-oleate & & 56.9 & 2.86 \\
\hline \multirow{2}{*}{$\begin{array}{l}\text { Trans-Epox-OOH- } \\
\text { linoleate }\end{array}$} & & A: 57.7 & 3.09 \\
\hline & & B: 60.4 & 2.81 \\
\hline \multirow{2}{*}{ Cis-Epox-OOH-linoleate } & & A: 56.2 & 3.40 \\
\hline & & & 3.04 \\
\hline
\end{tabular}

\subsubsection{Optimisation of quantitative epoxide assessment by HSQC}

\subsubsection{Acquisition parameters}

Firstly, the spectral range in the ${ }^{1} \mathrm{H}$ dimension was optimised. The signals that had to be included were the epoxy and TG signal regions, respectively resonating at $\delta_{\mathrm{H}} 3.3$ to $2.5 \mathrm{ppm}$ and $\delta_{\mathrm{H}} 4.4$ to $4.1 \mathrm{ppm}$. We compared the quality of HSQC spectra recorded with spectral ranges in the ${ }^{1} \mathrm{H}$ dimension from $\delta_{\mathrm{H}} 5$ to $1 \mathrm{ppm}$ and 9.5 to $0.5 \mathrm{ppm}$. Here, it was apparent that the tighter spectral range (Figure S3.1B) had improved resolution over the full spectral range (Figure S3.1A) and we decided to select $\delta_{\mathrm{H}} 5$ to $1 \mathrm{ppm}$ as spectral width. For the carbon dimension, narrowing the spectral width is not as straight forward. Since in HSQC, the protons are excited initially, which then transfer the magnetization to the carbon, cutting the ${ }^{13} \mathrm{C}$ NMR spectral width would result in folding in the ${ }^{13} \mathrm{C}$ dimension. A method to overcome this limitation is by using selective HSQC, which allowed only the recording of a certain ${ }^{13} \mathrm{C}$ NMR spectral range. Selective pulses are typically used to overcome two problems: resolution and dynamic-range limited sensitivity. We found an improved peak resolution for equal measurement time (Figures S3.1B/C) when employing selective pulses in the carbon 
dimension. However, since the TG peak was used as an internal standard, this region must be included which did not allow us to gain sensitivity by off-setting the dynamic range loss. So, even though there was an improvement on resolution, for the quantitative purpose of this method, we used the more accessible and widely used regular HSQC pulse sequence. In attempt to further reduce measurement time, non-uniform sampling (NUS) was explored. Here, we observed a loss in sensitivity when using NUS (data not shown) and decided not to venture this route further. This sensitivity loss was possibly due to the big dynamic range differences between the TG signals and the epoxy signals that hamper accurate reconstruction of the spectrum.

\subsubsection{Solvent selection}

In our previous work, we used a solvent mixture comprising of five units $\mathrm{CDCl}_{3}$ and one unit DMSO- $d_{6}$ to shift the ${ }^{1} \mathrm{H}$ NMR signals of hydroperoxides downfield to an isolated spectral region, allowing accurate quantification and identification (Merkx et al., 2018). For simultaneous detection of hydroperoxides, aldehydes, and epoxides, it would be convenient if the quantitative 2D HSQC approach works in this solvent system, without loss in sensitivity or the introduction of troublesome signal shifts. When comparing neat $\mathrm{CDCl}_{3}$ to the mixture of $\mathrm{CDCl}_{3}$ and DMSO- $d_{6}$ in a dilution series of epoxides ranging from 0.2 to $2 \mathrm{mmol}$ epoxides/kg oil, no significant differences were found between the epoxide concentrations ( $\mathrm{p}$ $>0.05$ ) (Figure 3.4). This implied that both the $\mathrm{CDCl}_{3}$ :DMSO- $d_{6}$ mixture as well as $\mathrm{CDCl}_{3}$ could be used depending on the analyst's needs. 


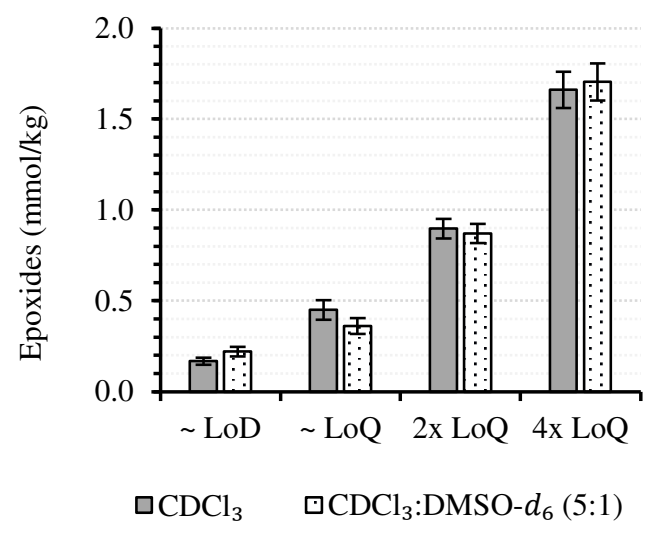

Figure 3.4. Impact of adding DMSO- $d_{6}$ to $\mathrm{CDCl}_{3}$ on the quantification of epoxides using HSQC. The LoD and LoQ displayed on the $\mathrm{x}$-axis correspond with the values in Table 3.2.

\subsubsection{Determination of K-factor}

As mentioned before, HSQC is not inherently quantitative since the signal peak volume is influenced by transversal and longitudinal relaxation times and $J_{\mathrm{CH}}$ and $\mathrm{J}_{\mathrm{HH}}$ coupling constants. These factors can be accounted for by a theoretical constant k, as described by Giraudeau (2014), but were difficult to determine experimentally for the signals of the glycerol backbone and epoxides. In this study, we opted for the use of a combined empirical $\mathrm{K}$-factor, which was used for direct quantification from the 2D HSQC spectrum (Equation 3.2). This K-factor was determined by quantitative comparison of the $1 \mathrm{D}$ signal integral and analogous 2D volume of the glycerol backbone and epoxide HSQC signals. To do so, the epoxy signals needed baseline-separation in both the 1D and 2D spectra. Hence, a fully chemically epoxidised rapeseed oil was used. Here, we assumed that the K-factor in chemically epoxidised oil was equal to that in thermally epoxidised oil. The K-factor was assumed equal for all type of epoxides regardless of their chemical environment. It is important to note that the K-factor is dependent on the experimental settings for both the $1 \mathrm{D}$ and 2D experiments. Henceforth, a K-factor of 1.16 was used in this study.

\subsubsection{Experimental validation and duration}

After determining the K-factor, method precision was determined by performing duplicate NMR experiments over different days on three epoxide concentrations. The results were 
analysed by ANOVA and expressed in terms of repeatability and reproducibility (respectively between- and within-day variation) (Table 3.2). The repeatability and reproducibility were close, indicating that error on within-day duplicates is similar to the between-day repetitions. The limit of quantification of individual epoxy signals was 0.6 $\mathrm{mmol} / \mathrm{kg}$ for an experimental duration of 70 minutes. Considering that typical shelf-life studies of food emulsions readily generate oxidation products above $2-5 \mathrm{mmol} / \mathrm{kg}$, the measurement time of the HSQC-experiment can be reduced to less than twenty minutes by decreasing the number of scans. When the whole epoxy region $\left(\delta_{\mathrm{C}} 61.3\right.$ to $52.3 \mathrm{ppm} ; \delta_{\mathrm{H}} 3.44$ to $2.38 \mathrm{ppm}$ ) was integrated instead of individual signals, the limits of detection and quantification were an order of magnitude higher. So, if the epoxide concentration is expected below this limit, it is advised to integrate the relevant peaks individually.

Table 3.2. Validation results for the quantification of epoxy moieties using ${ }^{1} \mathrm{H}^{13}{ }^{13} \mathrm{C}$ HQC NMR expressed by the limit of detection (LOD), limit of quantification (LOQ). The repeatability $\left(\mathrm{RSD}_{\mathrm{r}}\right)$ and reproducibility $\left(\mathrm{RSD}_{\mathrm{R}}\right)$ were determined at three epoxide concentrations.

\begin{tabular}{|c|c|c|c|c|}
\hline $\begin{array}{l}\text { Epoxide conc. } \\
(\mathrm{mmol} / \mathrm{kg})\end{array}$ & $\mathrm{RSD}_{\mathrm{r}}(\%)$ & $\operatorname{RSD}_{\mathrm{R}}(\%)$ & $\begin{array}{c}\mathrm{LoD} \\
(\mathrm{mmol} / \mathrm{kg})\end{array}$ & $\begin{array}{c}\text { LoQ } \\
(\mathrm{mmol} / \mathrm{kg})\end{array}$ \\
\hline 14.5 & 0.7 & 1.2 & \multirow{3}{*}{0.18} & \multirow{3}{*}{0.62} \\
\hline 1.35 & 4.1 & 4.0 & & \\
\hline 0.53 & 8.2 & 11.6 & & \\
\hline
\end{tabular}

\subsubsection{Formation and fate of epoxides during autoxidation of rapeseed oil and mayonnaise}

The optimised and validated method was applied to assess the impact of epoxides in accelerated shelf-life tests of relevant food products. Here, RP oil and mayonnaise were stored at both $40{ }^{\circ} \mathrm{C}$ and $60^{\circ} \mathrm{C}$. In these trials, a closed system was used to limit the maximum amount of available $\mathrm{O}_{2}$ and to enable the determination of the $\mathrm{O}_{2}$ consumption in the headspace. To evaluate whether epoxides had the potential as an early oxidation marker under accelerated shelf-life conditions, they were quantified using the newly developed method and compared to concentration of hydroperoxides and aldehydes. The generation of all oxidation products, in both RP oil and mayonnaises were accelerated when increasing the storage temperature from $40{ }^{\circ} \mathrm{C}$ to $60^{\circ} \mathrm{C}$. The same held when comparing RP oil with mayonnaise, with the latter having a lower oxidative stability. Due to the presence of catalytic iron and high interface area, emulsions are typically more prone to oxidation than oil (Berton, Ropers, 
Bertrand, Viau, \& Genot, 2012). Under all conditions, hydroperoxides were the most abundant lipid oxidation product that was formed (Figure 3.5), followed by epoxides, and finally aldehydes. A similar trend was described by Xia et al. (2015) in a storage test of soybean oil at $100{ }^{\circ} \mathrm{C}$. In both datasets (ours and by Xia et al. (2015)), hydroperoxides were formed in high amounts before the epoxides, which would render the latter less suitable as an early marker for lipid oxidation.

Mayonnaise

A

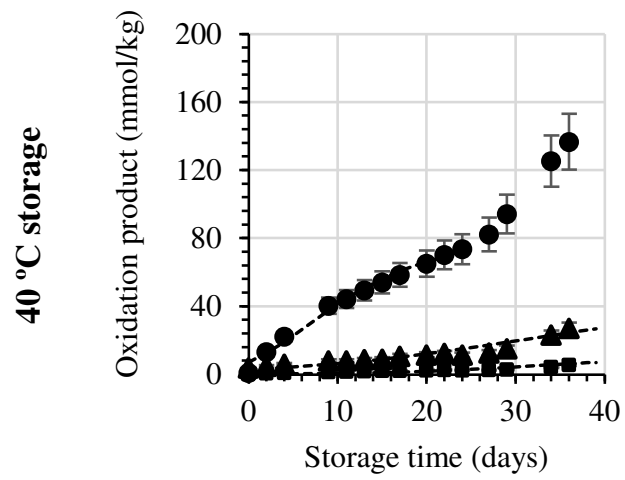

$\mathrm{C}$

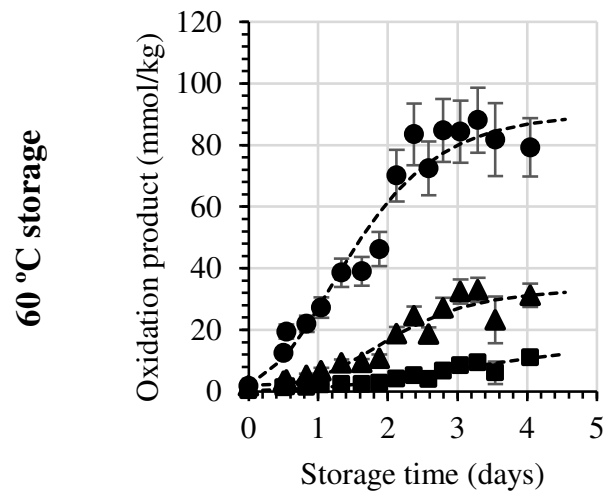

\section{RP Oil}

B

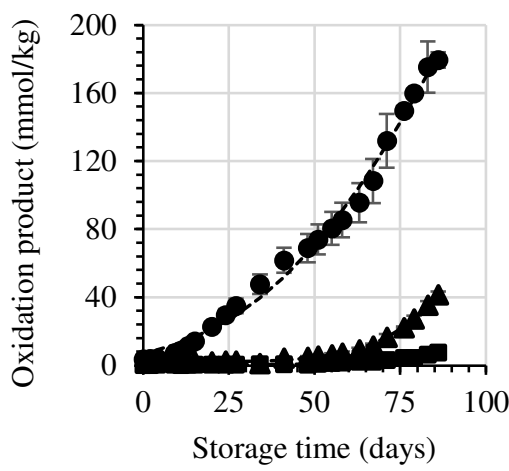

$\mathrm{D}$

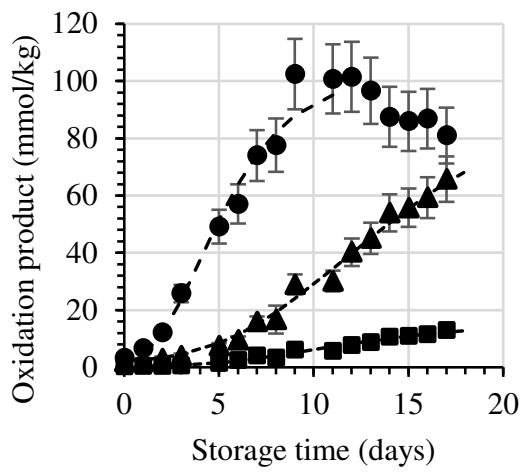

Figure 3.5. Formation of hydroperoxides (circles), epoxides (triangles) and aldehydes (squares) as function of storage time. A: Mayonnaise, stored at $40{ }^{\circ} \mathrm{C}, \mathrm{B}$ : RP oil, stored at $40^{\circ} \mathrm{C}, \mathrm{C}$ : mayonnaise, stored at $60^{\circ} \mathrm{C}, \mathrm{D}$ : RP oil, stored at $60^{\circ} \mathrm{C}$. Dotted lines are the sigmoidal Gompertz fits (Equation 3.8).

To further explore whether epoxides resemble early or late markers, we fitted the oxidation profiles with a sigmoidal model (Gompertz, 1825) and assessed the onset times $\left(\mathrm{t}_{0}\right)$ of the 
oxidation curves (Figure 3.6). We found that the assessment of accurate onset times for the $40{ }^{\circ} \mathrm{C}$ samples was impeded by lack of data at late oxidation stage, but since higher reaction rate at higher temperatures leads to a shorter onset time, we did manage to accurately capture the onset times at $60{ }^{\circ} \mathrm{C}$. Here, in the RP oil samples, the onset time of epoxide formation was more than twice longer compared to the one for hydroperoxide, whereas the difference between epoxides and aldehydes was low. This indicates that in the RP oil samples, the epoxides behaved more like late oxidation products (such as the aldehydes) than early oxidation products (such as hydroperoxides). In the mayonnaise samples at $60{ }^{\circ} \mathrm{C}$, this distinction was not observed. Here, both epoxides and aldehydes followed hydroperoxides kinetics much quicker, which could be explained by the role that iron plays in these systems. Iron is a known pro-oxidant that catalyses the degradation of hydroperoxides by $\mathrm{Fe}$ (II) to form $\mathrm{Fe}$ (III) and alkoxyl radicals (in a redox cycle), which then form both epoxides and aldehydes.

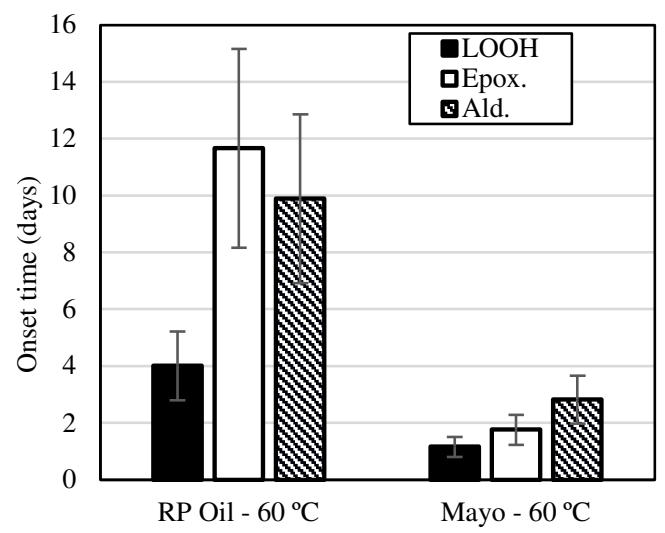

Figure 3.6. Plot of the onset times ( $\mathrm{t} 0$ ) obtained for the three lipid oxidation product classes by sigmoidal fitting (Gompertz model) for the samples stored at $60^{\circ} \mathrm{C}$. Displayed error bars are an empirically estimated value (30\%).

In this study, the oxygen consumption was determined by monitoring the amount of oxygen in the headspace to evaluate the contribution of epoxides to the total oxidation product range. At $40{ }^{\circ} \mathrm{C}$, the total oxygen consumption corresponded well with the sum of hydroperoxides, epoxides, and aldehydes expressed as mmol molecular oxygen per $\mathrm{kg}$ of oil, both in RP oils and mayonnaises (Figure 3.7A, B). Under these mild conditions, the contribution of hydroperoxides to the total oxidation products dominated those of the epoxides and aldehydes. For the mayonnaise stored at $60^{\circ} \mathrm{C}$, oxygen consumption was in agreement with formation of hydroperoxides, epoxides, and aldehydes in the early phase. At a later stage, the oxygen consumption was 40 to $60 \mathrm{mmol} / \mathrm{kg}$ higher than the total amount of detected oxidation products (Figure 3.7C, D), which was attributed to an alternative fate of the alkoxyl 
radicals, such as hydroxides, ketones, or polymers that are not accounted for in the current quantitative NMR measurements. Moreover, the used methods do not account for potential loss of volatiles, such as aldehydes. For the RP oil stored at $60^{\circ} \mathrm{C}$ (Figure 3.7D), the same was observed at the later stages. However, at the early stage (6 to 11 days), not all of the oxidation product formation could be accounted for by oxygen consumption, which we attributed to yet unaccounted experimental errors in the oxygen consumption method.

Mayonnaise

A

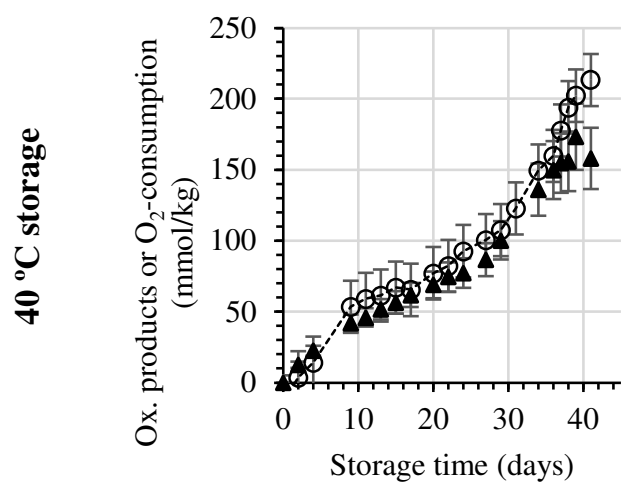

$\mathrm{C}$

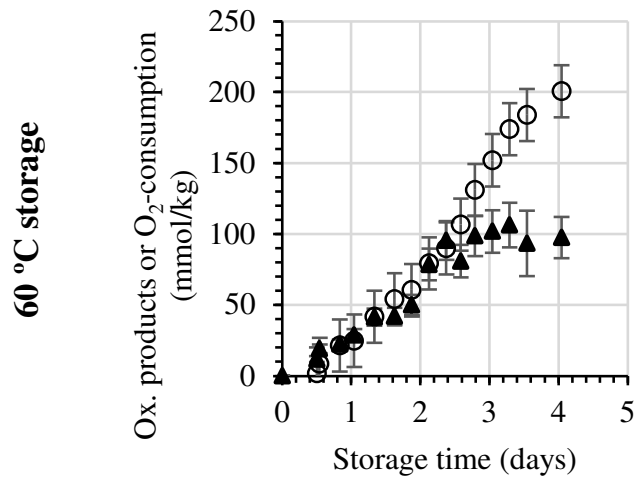

RP Oil

$\mathrm{B}$

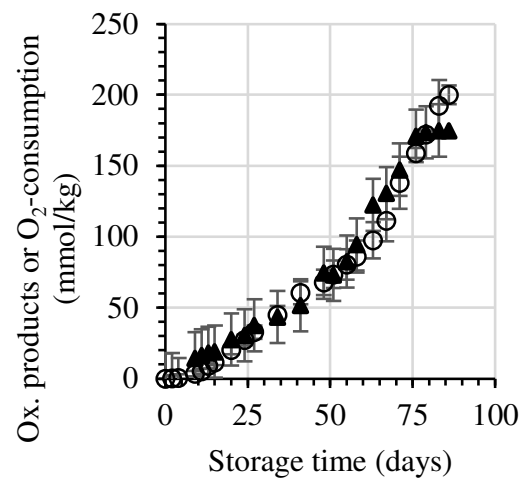

$\mathrm{D}$

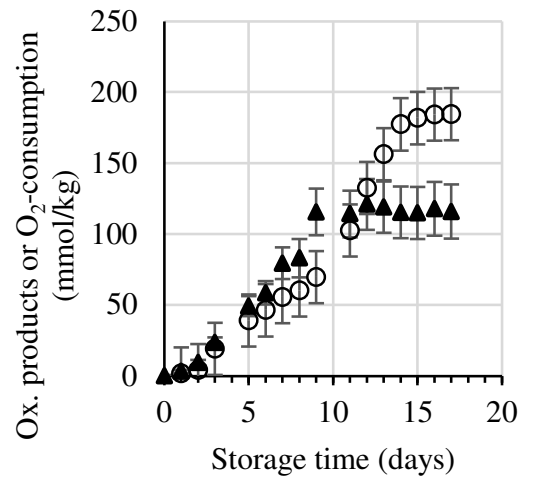

Figure 3.7. The oxygen consumption over time as determined in the sample headspace (open circles), and the sum of lipid hydroperoxides, epoxides, and aldehydes (closed triangles) that were generated as function of storage time. A: Mayonnaise, stored at $40{ }^{\circ} \mathrm{C}$, B: RP oil, stored at $40{ }^{\circ} \mathrm{C}$, C: mayonnaise, stored at $60{ }^{\circ} \mathrm{C}, \mathrm{D}: \mathrm{RP}$ oil, stored at $60^{\circ} \mathrm{C}$. The content of total oxidation products is expressed in mmol molecular oxygen per kg of oil. Hereto, the content of epoxy and aldehyde moieties is divided by two, as they only contain a single oxygen atom. 
The above results showed that in our test systems, epoxides are indeed an oxidation product that is often overlooked as it did show a major contribution (10-40\%) to the mass balance in the later stages of the oxidation. However, our results also indicated that the dominating mechanism for epoxide formation is via alkoxyl radical intermediates, similar as for aldehydes, which are considered a late oxidation product (Figure 3.8). To use epoxides as an early marker, they would need to be in competition with the hydroperoxides (via peroxyl radicals) rather than the aldehydes (via alkoxyl radicals), which was not the case in our tested systems. A combination of hydroperoxides, epoxides and aldehydes could, however, be promising to assess the relative contributions of the pathways mapped in Figure 3.8. This would provide a more comprehensive mechanistical view on lipid oxidation than either one of these markers alone.

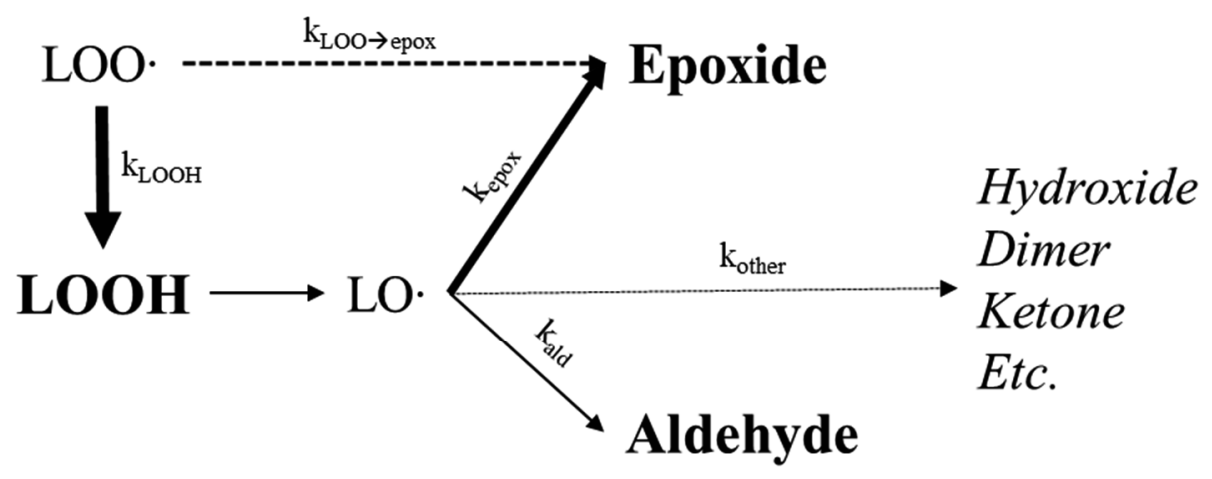

Fate of LOO. $\quad \mathrm{k}_{\mathrm{LOOH}} \gg \mathrm{k}_{\mathrm{LOO} \rightarrow \text { epox }}$

Fate of LO. $\quad \mathrm{k}_{\text {epox }}>\mathrm{k}_{\mathrm{ald}}>\mathrm{k}_{\mathrm{other}}$

Figure 3.8. A schematic interpretation of the results, illustrating the possible pathways to form epoxides. Compound classes in bold and italic were, respectively, measured and not measured in this investigation. The term ' $\mathrm{k}$ ' represents a qualitative interpretation of the kinetics as lumped rate constants. 


\subsection{Conclusion}

2D Heteronuclear Single Quantum Coherence (HSQC) can be used to quantify epoxides that are formed in thermal auto-oxidation in oil-based food systems. HSQC does not suffer from the significant signal overlap that complicates quantification of $1 \mathrm{D}$ spectra. The HSQC method has a high throughput since acquisition and processing can be automated. Furthermore, the reproducibility $(\mathrm{RSD}=11.6 \%)$, repeatability $(\mathrm{RSD}=8.4 \%)$, LoD $(0.18$ $\mathrm{mmol} / \mathrm{kg})$ and LoQ $(0.62 \mathrm{mmol} / \mathrm{kg})$ are adequate. The method is compatible with simultaneous quantification of hydroperoxides/aldehydes and is applicable in different solvent systems. The assignment of different subclasses of epoxides in the HSQC-spectrum enables the elucidation of mechanistical pathways. The method enables unravelling of the impact of storage temperature conditions in different matrices by considering oxidation pathways per compound class (hydroperoxides, epoxides, aldehydes) level. In mayonnaise and oils, epoxides are primarily formed via alkoxyl radical intermediates, which limits the potential of using solely epoxides as an early oxidation marker. However, in combination with hydroperoxides and aldehydes, epoxides can provide a comprehensive view on the relative pathway contributions.

\subsection{Literature}

Berton, C., Ropers, M.-H., Bertrand, D., Viau, M., \& Genot, C. (2012). Oxidative stability of oil-in-water emulsions stabilised with protein or surfactant emulsifiers in various oxidation conditions. Food Chemistry, 131(4), 1360-1369.

Cuvelier, M.-E., Soto, P., Courtois, F., Broyart, B., \& Bonazzi, C. (2017). Oxygen solubility measured in aqueous or oily media by a method using a non-invasive sensor. Food Control, 73, 1466-1473.

Fardus-Reid, F., Warren, J., \& Le Gresley, A. (2016). Validating heteronuclear 2D quantitative NMR. Analytical Methods, 8(9), 2013-2019.

Giraudeau, P. (2014). Quantitative 2D liquid-state NMR. Magnetic Resonance in Chemistry, 52(6), 259-272.

Goicoechea, E., \& Guillen, M. D. (2010). Analysis of Hydroperoxides, Aldehydes and Epoxides by $1 \mathrm{H}$ Nuclear Magnetic Resonance in Sunflower Oil Oxidized at 70 and $100{ }^{\circ} \mathrm{C}$. Journal of Agricultural and Food Chemistry, 58(10), 6234-6245.

Gompertz, B. (1825). On the Nature of the Function Expressive of the Law of Human Mortality, and on a New Mode of Determining the Value of Life Contingencies. Philos Trans. R. Soc. London, 115(513), 252-253.

Grüneis, V., Fruehwirth, S., Zehl, M., Ortner, J., Schamann, A., König, J., \& Pignitter, M. (2019). Simultaneous Analysis of Epoxidized and Hydroperoxidized Triacylglycerols in Canola Oil and Margarine by LC-MS. Journal of Agricultural and Food Chemistry, 67(36), 10174-10184. 
Grüneis, V., \& Pignitter, M. (2018). Epoxide Value-A Novel Marker for the Quality Assessment of Food Lipids. Journal of Agricultural and Food Chemistry, 66(20), 5039-5040.

Heikkinen, S., Toikka, M. M., Karhunen, P. T., \& Kilpeläinen, I. A. (2003). Quantitative 2D HSQC (Q-HSQC) via suppression of J-dependence of polarization transfer in NMR spectroscopy: application to wood lignin. J Am Chem Soc, 125(14), 4362-4367.

Lewis, I. A., Schommer, S. C., Hodis, B., Robb, K. A., Tonelli, M., Westler, W. M., . . . Markley, J. L. (2007). Method for determining molar concentrations of metabolites in complex solutions from two-dimensional 1H-13C NMR spectra. Anal Chem, 79(24), 9385-9390.

Liao, C.-H. (2013). Evaluation of assays for epoxides in oxidized lipids. MSc. Thesis, Rutger's University.

Merkx, D. W. H., Hong, G. T. S., Ermacora, A., \& van Duynhoven, J. P. M. (2018). Rapid Quantitative Profiling of Lipid Oxidation Products in a Food Emulsion by (1)H NMR. Anal Chem, 90(7), 4863-4870.

Peterson, D. J., \& Loening, N. M. (2007). QQ-HSQC: a quick, quantitative heteronuclear correlation experiment for NMR spectroscopy. Magn Reson Chem, 45(11), 937941.

Schaich, K. M., Xie, J., \& Bogusz, B. A. (2017). Thinking outside the classical chain reaction box of lipid oxidation: Evidence for alternate pathways and the importance of epoxides. Lipid Technology, 29(9-10), 91-96.

Skiera, C., Steliopoulos, P., Kuballa, T., Holzgrabe, U., \& Diehl, B. (2012). 1H-NMR Spectroscopy as a New Tool in the Assessment of the Oxidative State in Edible Oils. Journal of the American Oil Chemists' Society, 89, 1383-1391.

Van Duynhoven, J., van Velzen, E. \& Jacobs, D. M. . (2013). Quantification of complex mixtures by NMR. Annual Reports on NMR Spectroscopy, 80.

Xia, W., Budge, S., \& Lumsden, M. (2015). New 1H NMR-Based Technique to Determine Epoxide Concentrations in Oxidized Oil. Journal of Agricultural and Food Chemistry, 63.

Xia, W., \& Budge, S. M. (2017). Techniques for the Analysis of Minor Lipid Oxidation Products Derived from Triacylglycerols: Epoxides, Alcohols, and Ketones. Comprehensive Reviews in Food Science and Food Safety, 16(4), 735-758.

Xia, W., Budge, S. M., \& Lumsden, M. D. (2016). 1H-NMR Characterization of Epoxides Derived from Polyunsaturated Fatty Acids. Journal of the American Oil Chemists' Society, 93(4), 467-478. 


\subsection{Supporting Information}

Table S3.1. Integration ranges used in section 3.2.6.2. for the quantification of epoxides. Colours below correspond to the peaks annotated in Figure 3.3. Note that these values are the middle points of the integral ellipse and therefore can have a slight offset compared the values in Table 3.2.

\begin{tabular}{|ll|}
\hline $\begin{array}{l}\text { Integrated peaks } \\
\delta_{\mathrm{C}}(\mathrm{ppm})\end{array}$ & $\delta_{\mathrm{H}}(\mathrm{ppm})$ \\
\hline 46.7 & 3.07 \\
52.7 & 3.22 \\
52.9 & 3.13 \\
53.1 & 2.99 \\
54.1 & 2.73 \\
54.6 & 2.78 \\
55.2 & 3.52 \\
55.3 & 2.83 \\
55.6 & 3.47 \\
56.2 & 3.40 \\
56.6 & 2.88 \\
56.7 & 3.24 \\
\hline
\end{tabular}

\begin{tabular}{|ll|}
\hline \multicolumn{2}{|l|}{ Integrated peaks (continued) } \\
$\delta_{\mathrm{C}}(\mathrm{ppm})$ & $\delta_{\mathrm{H}}(\mathrm{ppm})$ \\
\hline 56.9 & 2.95 \\
57.1 & 2.80 \\
57.3 & 3.01 \\
57.5 & 3.17 \\
57.8 & 3.10 \\
58.3 & 2.67 \\
58.4 & 2.74 \\
58.4 & 3.05 \\
58.6 & 2.63 \\
59.5 & 3.19 \\
60.4 & 2.80 \\
61.2 & 2.89 \\
\hline
\end{tabular}


Table S3.2. Spectral assignment of the spectra shown in Figure 3.1. In parenthesis, the multiplicity is indicated, where $\mathrm{t}$ stands for a triplet, $\mathrm{m}$ for a multiplet, $\mathrm{q}$ for a quartet, and dd for a double doublet.

\begin{tabular}{lll} 
Signal & Chemical shift $(\mathrm{ppm})$ & Moiety \\
\hline $\mathrm{a}$ & $0.88(\mathrm{t})$ & Terminal methyl \\
$\mathrm{b}$ & $0.97(\mathrm{t})$ & Terminal methyl (omega-3) \\
$\mathrm{B}$ & $1.06(\mathrm{~m})$ & Terminal methyl at $\beta$-position epoxide \\
$\mathrm{c}$ & $1.3-1.25(\mathrm{~m})$ & $-\mathrm{CH}_{2}$ - (ethylene bridge) \\
$\mathrm{d}$ & $1.60(\mathrm{q})$ & $\mathrm{C} 3$ \\
$\mathrm{e}$ & $2.06-1.99(\mathrm{~m})$ & Allyl \\
$\mathrm{f}$ & $2.30(\mathrm{t})$ & $\mathrm{C} 2$ \\
$\mathrm{~g}$ & $2.79(\mathrm{t}) ; 2.76(\mathrm{t})$ & Bisallyl \\
$\mathrm{h}$ & $2.97(\mathrm{~m})$ & Monoepoxy \\
$\mathrm{i}$ & $3.19-3.06(\mathrm{~m})$ & Di/triepoxy \\
$\mathrm{j}$ & $4.30(\mathrm{dd}) ; 4.13(\mathrm{dd})$ & $\mathrm{CH}$ of glycerol backbone \\
$\mathrm{k}$ & $5.24(\mathrm{~m})$ & $\mathrm{CH}$ of glycerol backbone \\
$\mathrm{l}$ & $5.38-5.28(\mathrm{~m})$ & Vinyl
\end{tabular}

A

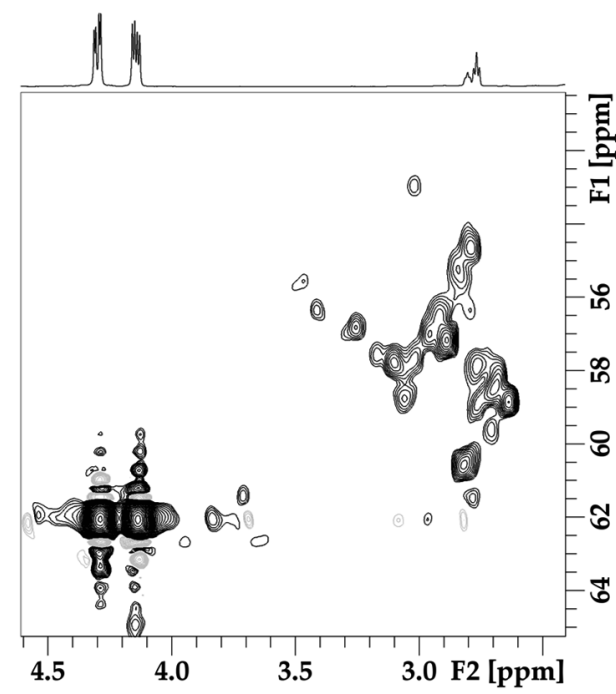

B

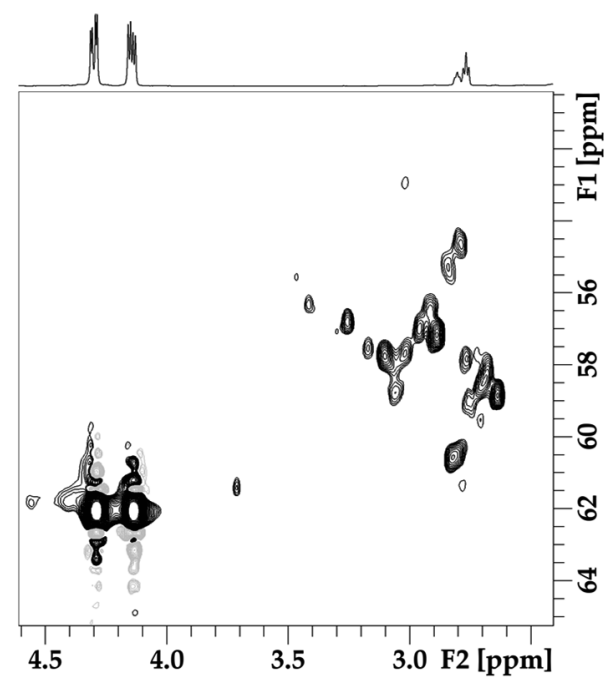

C 


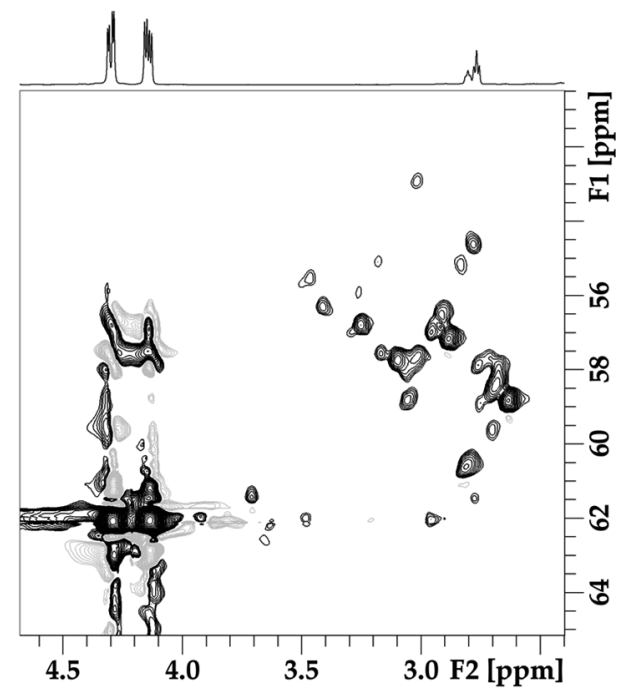

Figure S3.1. 2D HSQC NMR spectra $(600 \mathrm{MHz})$ of an auto-oxidised rapeseed oil recorded in full spectral width (A), limited spectral width in ${ }^{1} \mathrm{H}$ of $\delta 5$ to $1 \mathrm{ppm}$ and full spectral width in ${ }^{13} \mathrm{C}$ (B) and limited spectral width in ${ }^{1} \mathrm{H}$ of $\delta 5$ to $1 \mathrm{ppm}$ in combination with a selective pulse in the ${ }^{13} \mathrm{C}$ direction between $\delta 70$ to 50 ppm (C). In the F1 and F2 direction, the chemical shifts of respectively ${ }^{13} \mathrm{C}$ and ${ }^{1} \mathrm{H}$ are plotted. 
Figure S3.2. Details on the assignment of four major epoxides in thermally oxidised rapeseed oil as determined using 2D TOCSY-HSQC experiments.

\section{Oleate trans-epoxide}

\begin{tabular}{cccc} 
& & & 10 \\
\hline $\mathrm{C}$ & $\delta_{\mathrm{C}}(\mathrm{ppm})$ & $\delta_{\mathrm{H}}(\mathrm{ppm})$ & Correlation \\
\hline $7 \& 12$ & 25.9 & 1.32 & $8,9,10,11$ \\
$8 \& 11$ & 32.0 & 1.49 & $7,9,10,12$ \\
$9 \& 10$ & 58.6 & 2.62 & $7,8,11,12$ \\
\cline { 2 - 4 }
\end{tabular}

\section{Oleate cis-epoxide}

\begin{tabular}{llll}
\hline \#C & $\delta_{\mathrm{C}}(\mathrm{ppm})$ & $\delta_{\mathrm{H}}(\mathrm{ppm})$ & Correlation \\
\hline $7 \& 12$ & 26.5 & 1.34 & $8,9,10,11$ \\
$8 \& 11$ & 27.7 & 1.48 & $7,9,10,12$ \\
$9 \& 10$ & 56.9 & 2.86 & $7,8,11,12$ \\
\hline
\end{tabular}




\section{8:2 or 18:3 trans-epoxide-DB-OOH}

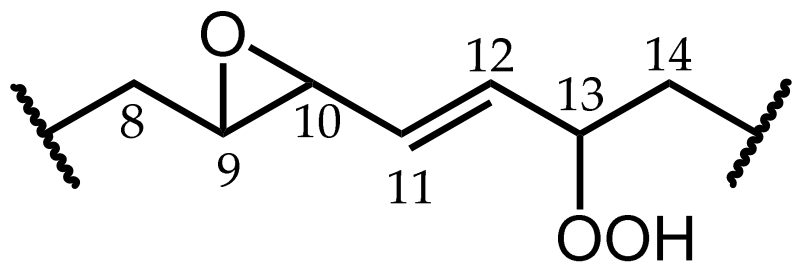

\begin{tabular}{llll}
\hline$\# \mathrm{C}$ & $\delta_{\mathrm{C}}(\mathrm{ppm})$ & $\delta_{\mathrm{H}}(\mathrm{ppm})$ & Correlation \\
\hline 8 & 31.8 & 1.32 & 9 \\
9 & 60.4 & 2.81 & 8,10 \\
10 & 57.7 & 3.09 & $9,11,12,13$ \\
$11 / 12$ & $134.8 / 130.7$ & $5.87 / 5.44$ & 10,13 \\
13 & 84.7 & 4.26 & $10,11,12,14$ \\
& & $1.43 ;$ & 13 \\
\hline
\end{tabular}

\section{8:2 or 18:3 cis-epoxide-DB-OOH}

\begin{tabular}{llll}
$\# \mathrm{C}$ & $\delta_{\mathrm{C}}(\mathrm{ppm})$ & $\delta_{\mathrm{H}}(\mathrm{ppm})$ & Correlation \\
\hline 8 & 27.7 & $?$ & 9 \\
9 & 58.6 & 3.04 & 8,10 \\
10 & 56.2 & 3.40 & $9,11,12,13$ \\
& 127.5 & 5.57 & \\
$11 \& 12$ & 136.1 & 5.88 & 10,13 \\
& 136.4 & 5.89 & \\
13 & 84.9 & 4.29 & 10 \\
\hline
\end{tabular}




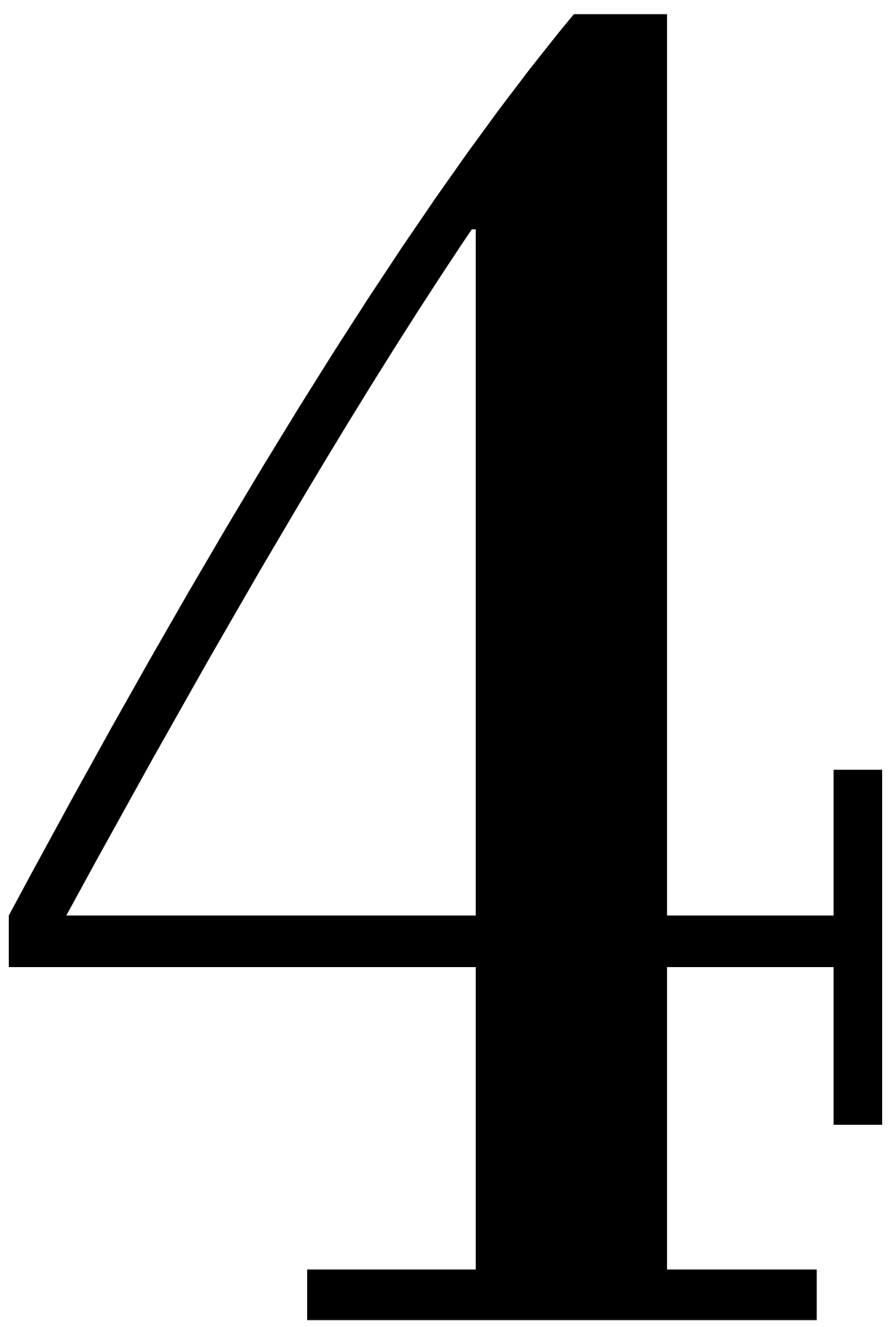




\section{Chapter 4}

\section{Evaluation of PBN spin-trapped radicals as early markers of lipid oxidation in mayonnaise}

Based on: Merkx, D. W. H., Plankensteiner, L., Yu, Y., Wierenga, P. A., Hennebelle, M., \& van Duynhoven, J. P. M. (2021). Evaluation of PBN spin-trapped radicals as early markers of lipid oxidation in mayonnaise. Food Chemistry, 334, 127578. 


\begin{abstract}
Quality deterioration of mayonnaise is caused by lipid oxidation, mediated by radical reactions. Assessment of radicals would enable early lipid oxidation assessment and generate mechanistic insights. To monitor short-lived lipid-radicals, N-tert-butyl- $\alpha$-phenylnitrone (PBN), a spin-trap, is commonly used. In this study, the fate of PBN-adducts and their impact on lipid oxidation mechanisms in mayonnaise were investigated. The main signals detected by Electron Spin Resonance (ESR) were attributed to L-radicals attached to 2-methyl-2nitrosopropane (MNP), one of three degradation products of the PBN-peroxy-adduct. The second degradation product, benzaldehyde, was detected with Nuclear Magnetic Resonance $\left({ }^{1} \mathrm{H}\right.$ NMR), in line with MNP-L adduct generation. For the third class of degradation products, LO-radicals, their scission products were detected with ${ }^{1} \mathrm{H}$ NMR and indicated that LO-radicals have a major impact on downstream oxidation pathways. This precludes mechanistical studies in presence of PBN. Degradation products of PBN-adducts can, however, be used for early assessment of antioxidants efficacy in oil-in-water emulsions.
\end{abstract}




\subsection{Introduction}

Lipid oxidation is one of the most detrimental reactions that can affect the quality of oil and oil-based emulsions. Lipid oxidation is a free radical chain reaction that is commonly divided into primary and secondary oxidation (Frankel, 2005). During primary oxidation, lipidradicals $(\mathrm{L} \cdot)$ react with oxygen $(\mathrm{O} 2)$ to form peroxy-radicals $(\mathrm{LOO} \cdot)$ that can abstract a hydrogen from other lipids (LH), generating hydroperoxides (LOOH). Secondary oxidation is commonly described as the degradation of these hydroperoxides into aldehydes, epoxides and other secondary oxidation products. These mechanisms are well described in bulk oil systems (Schaich, 2012; Schaich, Xie, \& Bogusz, 2017). In oil-in-water emulsions, the interfacial layer and transport of intermediates introduce an additional complexity to the lipid oxidation processes. (Berton-Carabin, Ropers, \& Genot, 2014; Laguerre, Tenon, Bily, \& Birtić, 2020). Elucidation of these mechanisms ideally requires quantitative measures on both primary and secondary oxidation products. Currently, several methods can quantitatively monitor the generation of primary and secondary oxidation products, such as peroxide value, ThioBarbituric Acid Reactive Substances (TBARS), chromatographic methods (Barriuso, Astiasarán, \& Ansorena, 2013; Steenhorst-Slikkerveer, Louter, Janssen, \& Bauer-Plank, 2000) and Nuclear Magnetic Resonance (NMR) (Merkx, Hong, Ermacora, \& van Duynhoven, 2018; Skiera, Steliopoulos, Kuballa, Holzgrabe, \& Diehl, 2012). These approaches, however, focus on stable products and are not designed to capture the radical intermediates, which are critical components for mechanistical understanding.

A technique that is commonly used to detect and monitor radicals is Electron Spin Resonance (ESR). However, the radicals that are formed during lipid oxidation are highly reactive with very low steady state concentrations, which prevent direct detection by ESR. For such analyses, spin-traps can be used, which are molecules that react with free radicals, effectively stabilizing the radicals for a sufficiently long time (Andersen \& Skibsted, 2006). The sensitivity of ESR is $~ 1000$ times higher than NMR, potentially allowing oxidative assessment at even earlier time points. To investigate radicals in oil-in-water emulsions, the spin-trap should be stable at mildly accelerated shelf-life conditions, soluble in oil and should not be too expensive to use in shelf-life studies. A spin-trap that meets these requirements is N-tert-butyl- $\alpha$-phenylnitrone (PBN). PBN is widely used for lipid oxidation studies (Boerekamp, Andersen, Jacobsen, Chronakis, \& García-Moreno, 2019; Cui, Lahti, \& Decker, 2016; Thomsen, Jacobsen, \& Skibsted, 2000; Thomsen, Kristensen, \& Skibsted, 2000; Thomsen, Vedstesen, \& Skibsted, 1999; Velasco, Andersen, \& Skibsted, 2004). Despite its wide use, its fate and potential impact on lipid oxidation mechanisms have not been investigated in detail. It has been reported that PBN rapidly degrades both in model systems at mild conditions (Janzen, Krygsman, Lindsay, \& Haire, 1990; Vicente et al., 1998), as well 
as in oil under extreme thermal stress $\left(180^{\circ} \mathrm{C}\right)$ (Silvagni, Franco, Bagno, \& Rastrelli, 2010). Under these circumstances, $\mathrm{PBN}$ is reported to react with the LOO radicals, forming LOOPBN, which rapidly decomposes to alkoxy-radicals (LO·), benzaldehyde and 2-methyl-2nitrosopropane (MNP) (Janzen et al., 1990; Silvagni et al., 2010). Here, MNP acts as a secondary spin-trap that reacts with L- radicals to form the MNP-L adducts that are actually measured with ESR. At this point, whether this PBN degradation mechanism is the same in food emulsions in milder storage conditions has not been investigated. It would limit the applicability of spin traps for early diagnostics of lipid oxidation (Velasco, Andersen, \& Skibsted, 2005) and unravelling of lipid oxidation mechanisms.

In this work, we will first establish the PBN reaction pathways in rapeseed oil under modest shelf-life conditions. Next, these pathways will be verified in mayonnaise where the modulation of lipid oxidation mechanism by PBN will be studied. This will also involve the interaction of PBN reaction products with the antioxidant mechanisms of the metal chelator EDTA. Finally, we will assess the applicability and limits of using ESR for the early assessment of antioxidant efficacy.

\subsection{Material and Methods}

\subsubsection{Materials}

N-tert-butyl-a-phenylnitrone (PBN, 99.5\%), 2-methyl-2-nitrosopropane dimer (MNP dimer) and 16-doxyl-stearic acid (16-DSA), ethylenediaminetetraacetic acid calcium disodium salt ( $\mathrm{CaNa}_{2}$ EDTA) and benzaldehyde (99\%) were purchased from Sigma Aldrich (Zwijndrecht, the Netherlands). $\mathrm{CDCl}_{3}, \mathrm{DMSO}-\mathrm{d}_{6}$ and $4 \AA$ molecular sieves were purchased from Eurisotop (Saint-Aubin, France). To facilitate solubility, PBN was ground before use using a pestle and mortar. Rapeseed (RP) oil, Medium Chain Triglyceride (MCT) oil, egg yolk, sodium chloride, sugar and starch were purchased from local suppliers. Alumina powder (MP EcoChrome $^{\mathrm{TM}}$ ALUMINA N, Activity: Super I, Biomedicals) was purchased from MP Biomedicals (Irvine, USA).

\subsubsection{Rapeseed oil experiments}

PBN was directly dissolved in the rapeseed oil in a glass container. To speed up the dissolution, the oil was heated for $30 \mathrm{~min}$ at $30^{\circ} \mathrm{C}$. Two experimental series were performed on rapeseed oil samples. For the oxygen-availability series, $50 \mathrm{~mL}$ solutions of $40 \mathrm{mM}$ of PBN in rapeseed oil were prepared. The oil was separated into two $22 \mathrm{~mL}$ aliquots in $50 \mathrm{~mL}$ Falcon tubes (polypropylene). For the oxygen-rich sample, the Falcon tube was filled with 
atmospheric air and closed with a lid. For the oxygen-poor sample, the Falcon tube was bubbled continuously with nitrogen (no lid). The tubes were stored 7 days in a $50{ }^{\circ} \mathrm{C}$ water bath to accelerate the oxidation of the rapeseed oil. From both tubes, in duplicate, $1 \mathrm{~mL}$ aliquots were taken every day and frozen at $-80{ }^{\circ} \mathrm{C}$ until further use.

For the PBN-concentration series, $50 \mathrm{~mL}$ solutions of 10, 40 and $60 \mathrm{mM}$ of PBN in rapeseed oil were prepared. From this, $1 \mathrm{~mL}$ was transferred to $1.5 \mathrm{~mL}$ Eppendorf tubes (polypropylene) and stored in an oven at $40{ }^{\circ} \mathrm{C}$ up to 12 days. Samples were taken in duplicates at each time point and directly frozen at $-80{ }^{\circ} \mathrm{C}$ until further use.

\subsubsection{Mayonnaise production and storages}

All mayonnaises were prepared by using a Silverson mixer (Chesham, United Kingdom) for emulsification in $400 \mathrm{~g}$ batches. Rapeseed oil was used as such or stripped with alumina powder (MP EcoChrome ${ }^{\mathrm{TM}}$ ALUMINA N, Activity: Super I, Biomedicals) to remove impurities and tocopherols (Berton, Ropers, Viau, \& Genot, 2011), this to prevent endogenous anti-oxidants interfering with the oxidation process. A $6.7 \mathrm{mM}$ EDTA stock solution was prepared by dissolving $250 \mathrm{mg}$ calcium disodium EDTA in $100 \mathrm{~mL}$ demineralised water. Two sets of experiment were performed with two types of formulations as described below.

\subsubsection{Quantitative assessment of radical formation in a model mayonnaise}

The mayonnaise samples were prepared with 0 or $0.2 \mathrm{mM}$ EDTA (respectively E- and E+) and contained $78 \%$ of stripped rapeseed (SRP) oil. The SRP oil was devoid of native antioxidants and thus more prone to oxidation. The water phase $(82.4 \mathrm{~g})$ was prepared first. For this, $20 \mathrm{~g}$ egg yolk, $4.4 \mathrm{~g}$ sodium chloride, 0 or $3 \mathrm{~g}$ EDTA-solution and 14.5 or $11.5 \mathrm{~g}$ demineralised water were mixed in a $500 \mathrm{~mL}$ plastic container. Next, $312 \mathrm{~g}$ SRP oil was slowly added to form the emulsion and finally $5.6 \mathrm{~g}$ spirit vinegar was added to achieve the desired $\mathrm{pH}$ (3.8). To $120 \mathrm{~g}$ of both formulations (E- and $\mathrm{E}+$ ), $1600 \mu \mathrm{L} 1.5 \mathrm{M}$ PBN in DMSO (details in S4.1) was post-added to prepare $20 \mathrm{mM}$ PBN formulations (E-P+ and $\mathrm{E}+\mathrm{P}+$ ). Aliquots of $1 \mathrm{~g}$ were stored in $20 \mathrm{~mL}$ screwcap vials in the dark at $40{ }^{\circ} \mathrm{C}$. Aliquots were removed from storage in duplicate for a total of 57 days with a high sampling density during the first ten days, since we expected to see most PBN-activity during the early oxidation phase. The aliquots were then stored at $-20{ }^{\circ} \mathrm{C}$ until further analysis. For all samples, ${ }^{1} \mathrm{H}$ NMR was used to monitor the generation of $\mathrm{LOOH}$ and n-alkanals) as representatives for respectively primary and secondary lipid oxidation. MNP-L spin-adduct and benzaldehyde generation were respectively measured by ESR and ${ }^{1} \mathrm{H}$ NMR for the samples with PBN. 


\subsubsection{Early assessment of antioxidant protection against primary oxidation in a full mayonnaise.}

For the assessment on the oxidative stability in mayonnaises, mayonnaise samples were prepared with four levels of EDTA $(0,0.03,0.06$ and $0.20 \mathrm{mM})$ and a $65 \%$ oil-content. The water phase (113.6 g) was prepared first. For this, $16.8 \mathrm{~g}$ egg yolk, $4.4 \mathrm{~g}$ sodium chloride, 4 $\mathrm{g}$ starch, $5.2 \mathrm{~g}$ sugar, 0 to $3 \mathrm{~g}$ EDTA-solution and 24.8 to $21.8 \mathrm{~g}$ demineralised water were mixed in a $500 \mathrm{~mL}$ plastic container. Next, $260 \mathrm{~g}$ rapeseed oil was slowly added to form the emulsion and finally $10.4 \mathrm{~g}$ spirit vinegar was added to achieve the desired $\mathrm{pH}$ (3.8). For the NMR-assessment, aliquots of $1 \mathrm{~g}$ were stored in $20 \mathrm{~mL}$ screwcap vials at $50{ }^{\circ} \mathrm{C}$. Aliquots were removed from storage in duplicate at set intervals (0, 1, 4 and 6 days) and stored at -20 ${ }^{\circ} \mathrm{C}$ until further analysis. For the ESR-assessment, $50 \mu \mathrm{L}$ of $700 \mathrm{mM}$ PBN solution (PBN in MCT oil, details in S4.1) was added to $5 \mathrm{~g}$ aliquots, in triplicate for each formulation, in 15 $\mathrm{mL}$ Falcon tubes (polypropylene) and gently stirred. The mayonnaises were incubated at 50 ${ }^{\circ} \mathrm{C}$ for 5 hours. During incubation, aliquots of mixtures were collected in triplicate at set intervals $\left(0,1.5,3\right.$ and 5 hours) and stored at $-80^{\circ} \mathrm{C}$ until further analysis.

\subsubsection{Electron Spin Resonance (ESR)}

Frozen mayonnaise samples were thawed at room temperature, resulting in separation between the oil and the water phase. The sample (oil or oil-layer of the mayonnaise) was transferred to a $200 \mu 1$ micropipette capillary to a minimal filling height of $4 \mathrm{~cm}$. The capillary was placed in a quartz ESR tube of $5 \mathrm{~mm}$ diameter and subsequently moved into the ESR cavity. The ESR spectra were recorded with an X-band ESR spectrometer (Elexsys E 500, Bruker, Rheinstetten, Germany) running at $9.32 \mathrm{GHz}$. For the acquisition, the microwave power was set at $13.72 \mathrm{~mW}$ the modulation amplitude at $3.0 \mathrm{G}$, with the center field at 3328 $\mathrm{G}$ and a sweep width of $100 \mathrm{G} .1024$ number of points were used with both a conversion time and time constant of 164 . The acquisition settings were kept constant for all quantification measurements, only the receiver gain was adjusted $(20-60 \mathrm{~dB})$ and the outcome of the measurement was accordingly corrected. Generally, spectra were recorded at room temperature. For the identification of the spin adducts, the spectra were recorded at $70{ }^{\circ} \mathrm{C}$ in order to increase the molecular mobility (Thomsen et al., 1999) and the modulation amplitude was reduced up to 0.04 to prevent spectral line broadening. The trapped radicals were quantified with a calibration curve $(0-5 \mathrm{mM})$ of the stable radical 16-DSA. The analysis of the ESR spectra was carried out with the software package Easyspin (Stoll \& Schweiger, 2006), running in a MATLAB (version R2017b, MathWorks, USA) environment. The spectra were imported and after applying a smoothing function, the double integration was 
performed. Detected spin adducts were only quantified if the signal to noise ratio was higher than 10 .

\subsubsection{Nuclear Magnetic Resonance (NMR)}

For the quantification of oxidation products, the (separated) oil was dissolved in a 5:1 $\mathrm{CDCl}_{3}$ :DMSO-d6 solvent. For the benzaldehyde assignment, $25 \mu \mathrm{L}$ pure benzaldehyde was added to $600 \mu \mathrm{L}$ of the mixture. Single pulse and band selective ${ }^{1} \mathrm{H}$ NMR spectra were recorded on a $600 \mathrm{MHz}(14.1 \mathrm{~T})$ Bruker Avance III NMR spectrometer (Bruker BioSpin, Switzerland) according to the protocol described in earlier work (Merkx et al., 2018). The spectrum obtained by single pulse excitation was used for the quantification of PBN $(\delta 8.3$ ppm), triglyceride $(\delta 4.4 \mathrm{ppm})$ and optionally benzaldehyde $(\delta 7.5 \mathrm{ppm})$. The triglyceride (TG) peak area was used for the internal calibration for the quantification of the hydroperoxides, aldehydes and benzaldehyde. For the band selective experiment, the region between $\delta 13.0$ and $8.0 \mathrm{ppm}$ was selectively excited. Hydroperoxides, aldehydes and benzaldehyde were quantified via integration of the signal regions between $\delta 11.2-10.7 \mathrm{ppm}$, $\delta$ 9.8-9.2 ppm and $\delta$ 10.1-9.9 ppm, respectively. Details on calculations of concentrations from the integral regions in the single pulse and selective excitation experiment are described in previous work (Merkx et al., 2018).

\subsubsection{Statistical analysis}

ANOVA tests were performed, followed by Bonferroni corrected two-tailed T-tests to assess the significance of the differences between samples and differences over time. All tests were performed using Microsoft Excel. Statistical significance was set at $\mathrm{p}<0.05$.

\subsection{Results and Discussion}

\subsubsection{Identification and quantification of spin-trapped radicals in rapeseed oil}

The mechanism of PBN spin trapping was first investigated in pure rapeseed oil. Rapeseed oil was the main ingredient of mayonnaise and its use simplified the sample handling and reduced the mechanistic complexity. This trial was done using two oxygen conditions (i.e. oxygen-rich and oxygen-depleted) to explore the PBN spin trapping mechanisms in the most opposing scenarios (Silvagni et al., 2010) in mild storage conditions. Under oxygen rich conditions, an ESR signal was observed after 24 hours at $50^{\circ} \mathrm{C}$. Three hyperfine lines $\left(\mathrm{I}_{\mathrm{N}}=\right.$ 1) with a hyperfine coupling constant $\left(\mathrm{a}_{\mathrm{N}}\right)$ of $14.8 \mathrm{G}$ were clearly distinguished (Figure $4.1 \mathrm{~A}$ ). 
This coupling constant $(14.8 \mathrm{G})$ suggests the presence of a nitroxyl group. After increasing the temperature in the ESR tube to $70{ }^{\circ} \mathrm{C}$ to increase the molecular mobility, the three hyperfine lines were resolved into six hyperfine lines. A second hyperfine interaction was visible with a hyperfine coupling constant $\left(\mathrm{a}_{\mathrm{H}}\right)$ of $2.1 \mathrm{G}$ (Figure 4.1B). This combination of hyperfine coupling constants corresponds to MNP-L adducts (Vicente et al., 1998). Importantly, no superposition of hyperfine splittings that could indicate the presence of other spin adducts (e.g. MNP-OOL or PBN-L) were observed in the ESR-spectra. The sample was cooled down and measured again to verify that short heating to $70{ }^{\circ} \mathrm{C}$ did not irreversibly generate a new spin adduct. Here, the same spectrum as before the heating was observed (data not shown), indicating that the same molecule was measured at room temperature and at $70^{\circ} \mathrm{C}$. To confirm the identification of MNP-L adducts, MNP-dimer was added to rapeseed oil and stored at $40^{\circ} \mathrm{C}$. As expected, the MNP-adducts that were observed with ESR showed identical hyperfine splittings to those described above (Figure S4.2).

Under oxygen depleted conditions, the ESR spectra (Figure 4.1C) clearly showed a different hyperfine splitting than the one observed under oxygen rich condition (Figure 4.1A/B). The coupling constants were $14.5 \mathrm{G}$ and $3.2 \mathrm{G}$ for respectively $\mathrm{a}_{\mathrm{N}}$ and $\mathrm{a}_{\mathrm{H}}$. These coupling constants were assigned to the spin adducts between PBN and alkyl radicals (PBN-L) (Vicente et al., 1998). This observation matched with our hypothesis that under oxygen poor conditions, no PBN-OOL spin adducts were generated that could decompose to form MNP and subsequently MNP-L adducts, as observed under oxygen rich condition. 

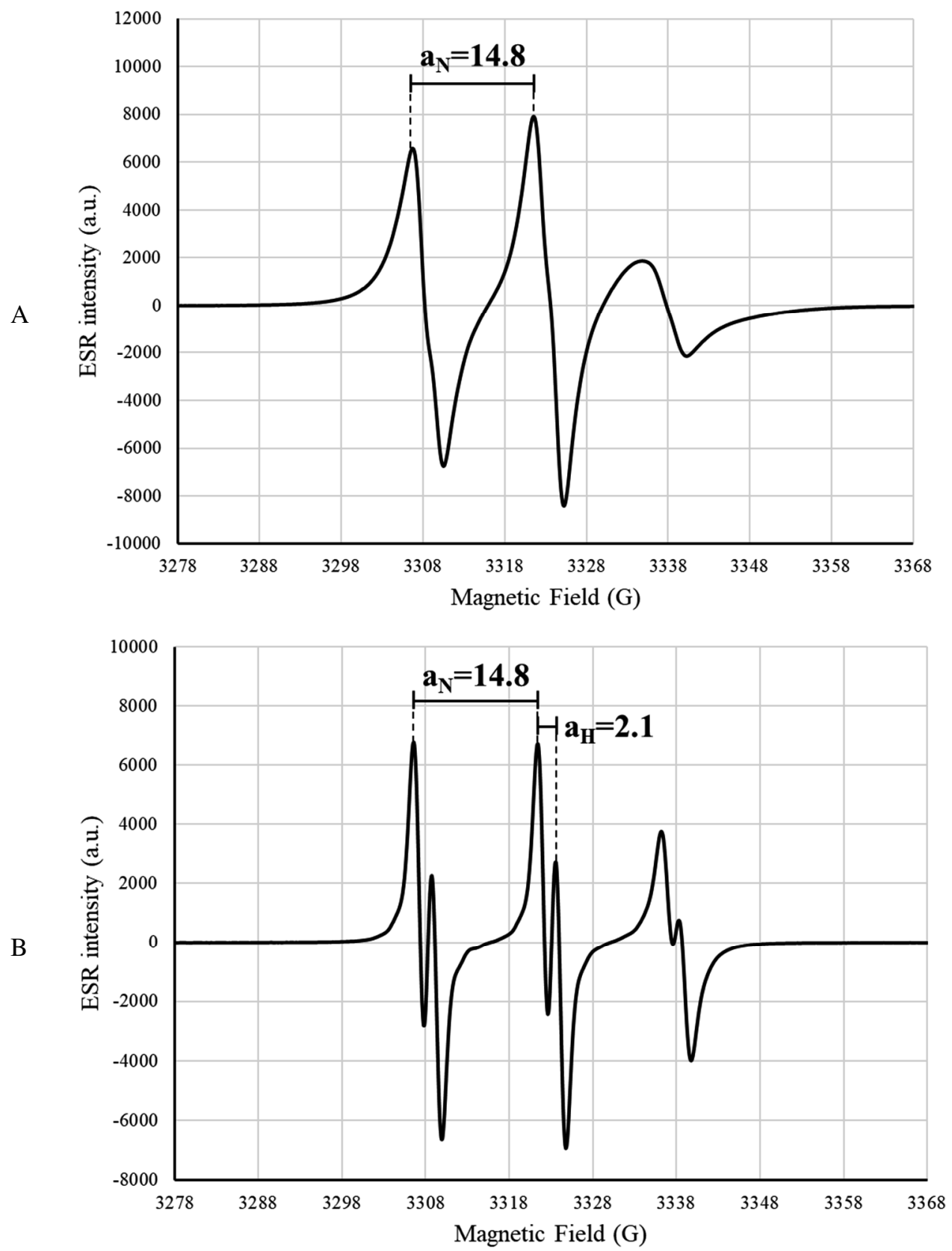


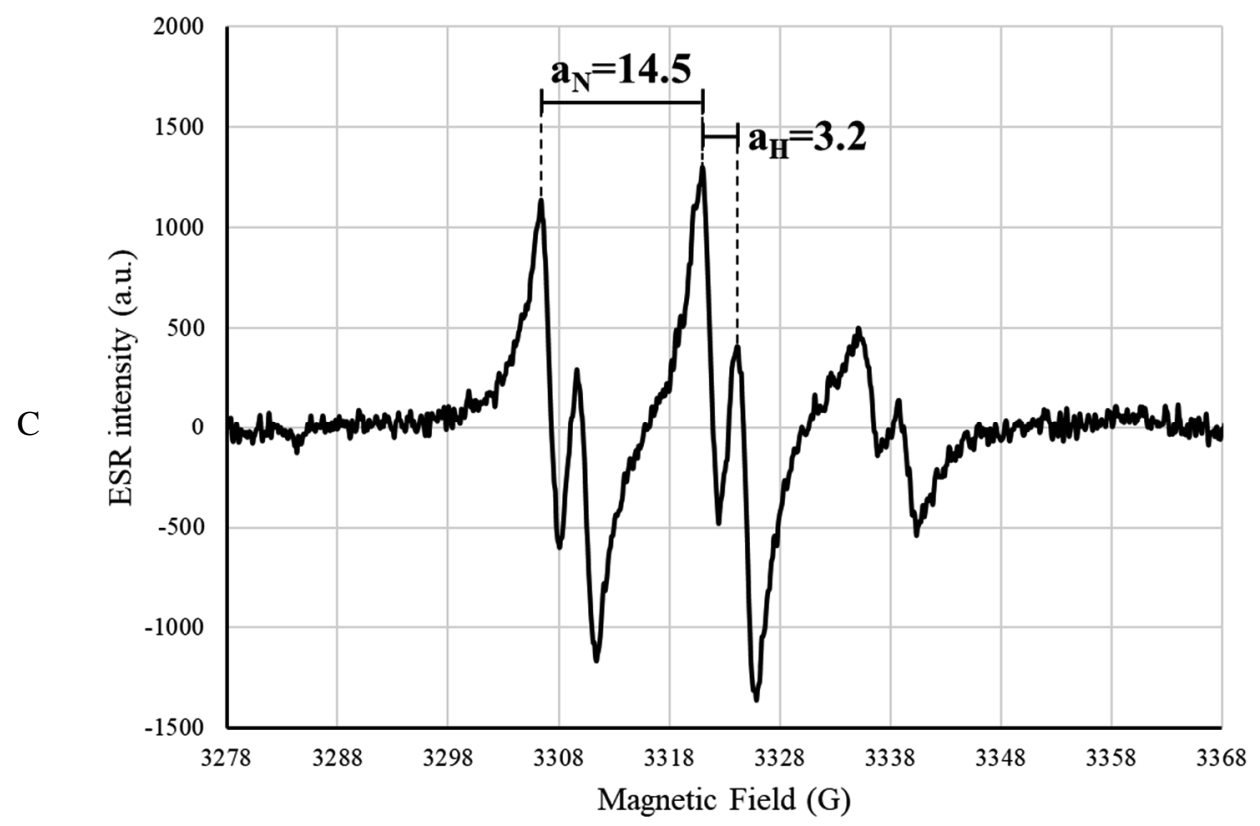

Figure 4.1. ESR spectra of spin adducts in rapeseed oil after PBN addition $(40 \mathrm{mM})$ and heated storage $\left(50{ }^{\circ} \mathrm{C}\right.$ for 7 days). A: ESR spectrum of rapeseed oil stored at $50{ }^{\circ} \mathrm{C}$ under aerobic conditions and measured at room temperature; B: ESR spectrum of rapeseed oil stored at $50{ }^{\circ} \mathrm{C}$ under aerobic conditions and measured at $70{ }^{\circ} \mathrm{C}$; $\mathrm{C}$ : ESR spectrum of nitrogen bubbled rapeseed oil stored at $50{ }^{\circ} \mathrm{C}$ and measured at room temperature.

The previously reported degradation mechanisms of PBN-OOL to MNP implied the simultaneous formation of benzaldehyde (Janzen et al., 1990). Hence, we verified the appearance of benzaldehyde by ${ }^{1} \mathrm{H}$ NMR. In the ${ }^{1} \mathrm{H}$ NMR spectrum of rapeseed oil with 40 mM PBN, a carbonyl proton signal was detected at $\delta 10.15 \mathrm{ppm}$. To verify the assignment of the carbonyl peak to benzaldehyde, we performed a spiking experiment with pure benzaldehyde and a 2D TOCSY experiment (Assignment in S4.3). The signal of the carbonyl proton of benzaldehyde $(\delta 10.15 \mathrm{ppm})$ resonated between the hydroperoxide $(\mathrm{LOOH})$ region and aldehyde regions. This allowed for benzaldehyde quantification $(\mathrm{LoQ}=0.05 \mathrm{mM}) \mathrm{using}$ the same band-selective pulse used for exciting the $\mathrm{LOOH}$ and aldehyde regions, as described in our previous work (Merkx et al., 2018).

Combining assessments of MNP-L or PBN-L by ESR and benzaldehyde by ${ }^{1} \mathrm{H}$ NMR allowed for a multifaceted approach to monitor the fate of PBN-OOL. After 7 days under oxygendepleted conditions, around $2 \mu \mathrm{M}$ of PBN-L and no benzaldehyde were detected. In the absence of oxygen, little to no LOO radicals were formed to consequently form benzaldehyde (via PBN-OOL). This enabled PBN to directly react with the $\mathrm{L} \cdot$ to form $\mathrm{PBN}$ $\mathrm{L}$ in low amounts. Under oxygen-rich conditions, concentrations of $360 \mu \mathrm{M}$ MNP-L and 800 $\mu \mathrm{M}$ benzaldehyde was reached (Figure S4.4). The benzaldehyde and MNP-L levels after 168 
hours differed by approximately a factor of two. This difference was attributed to higher stability of benzaldehyde than MNP-L. Benzaldehyde was not expected to degrade within these 168 hours, whereas MNP-L is a radical species that is prone to reduction and consequently a loss of ESR signal. Moreover, the MNP-L spin adducts showed a lag time of 24 hours compared to benzaldehyde. Indeed, in order to form the MNP-L spin adducts, MNP needed to compete with $\mathrm{O}_{2}$ to react with $\mathrm{L}$.

This experiment showed that under mild storage conditions, MNP-L is indeed the spin-adduct that is detected with ESR when PBN was initially added (Figure 4.2). The MNP-L adducts are a product of the free alkyl radicals $(\mathrm{L} \cdot)$ with $\mathrm{MNP}$, which in turn is a decomposition product of the PBN-OOL adducts. Benzaldehyde, the by-product of PBN-OOL decomposition can be quantified by ${ }^{1} \mathrm{H}$ NMR and thereby can be used as a direct marker and proof of PBN-OOL decomposition and as an indirect marker of LOO-generation.

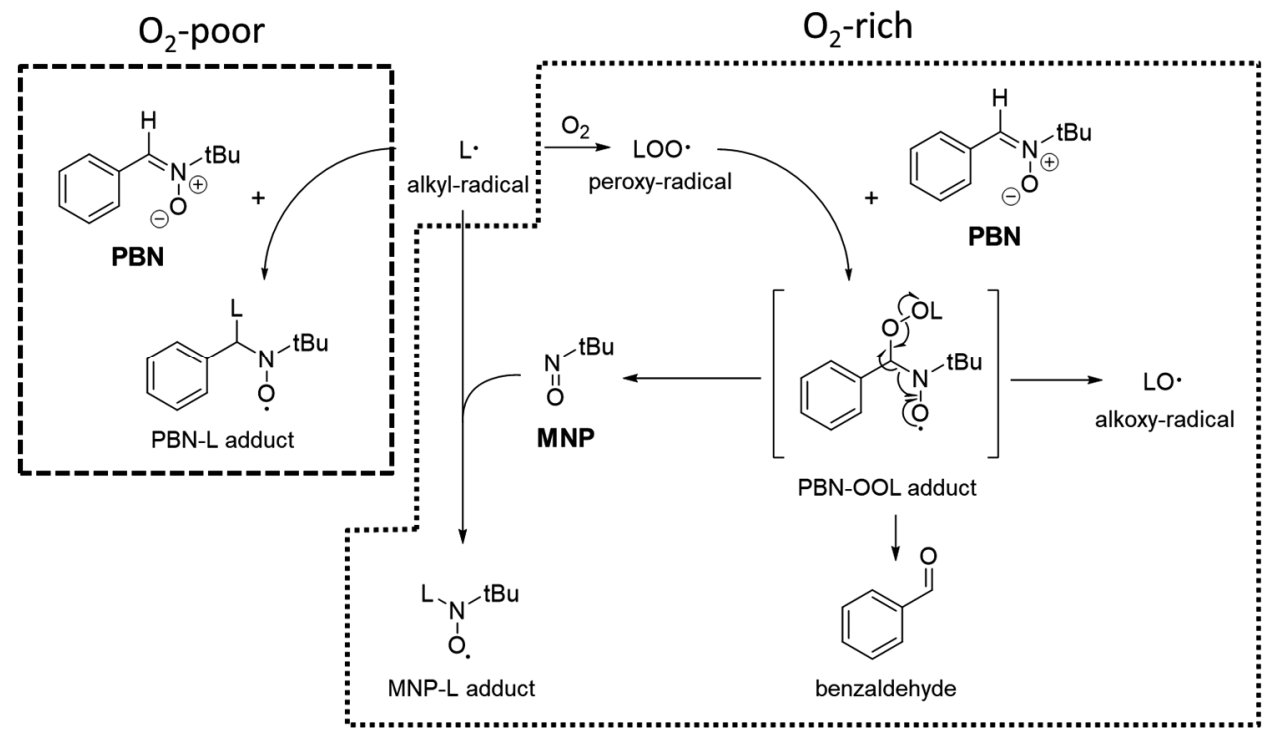

Figure 4.2. Proposed mechanisms of the interaction of PBN with different radicals and the fate of its products in vegetable oils and emulsions. The right part of the diagram is in oxygen rich conditions, the left part under oxygen depleted conditions.

Next, the effect of the PBN concentration on the formation of the MNP-L adducts and benzaldehyde was evaluated. As expected, in absence of PBN, no MNP-L and benzaldehyde were formed. In the first 200 hours, increasing the PBN concentration up to $60 \mathrm{mM} \mathrm{PBN}$, resulted in a proportional increase of MNP-L and benzaldehyde to respectively $0.16 \mathrm{mM}$ and $0.25 \mathrm{mM}$ (Figure 4.3). Thus, the total amount of MNP-L and benzaldehyde depended on the concentration of added PBN. This dependence should be considered when interpreting MNP$\mathrm{L}$ and benzaldehyde concentrations. Furthermore, it complicates the quantitative comparison between experimental series with different PBN concentrations. More importantly, the 
increase in the amount of MNP and benzaldehyde with increasing PBN concentration is likely accompanied by an increase in LO concentration, which can contribute to the generation of more $\mathrm{L}$ - and LOO', and consequently of more PBN-OOL, MNP and benzaldehyde. This concentration-dependence clearly shows that PBN addition perturbs the oxidative reaction mechanism in rapeseed oil. This limits the applicability of spin-trapping as quantitative tool to link the measured spin adducts to other oxidation products, such as LOOHs and aldehydes for e.g. mass balancing purposes.

A

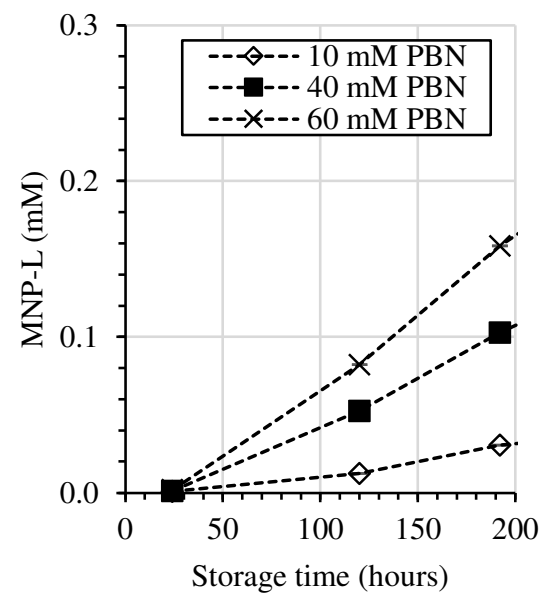

B

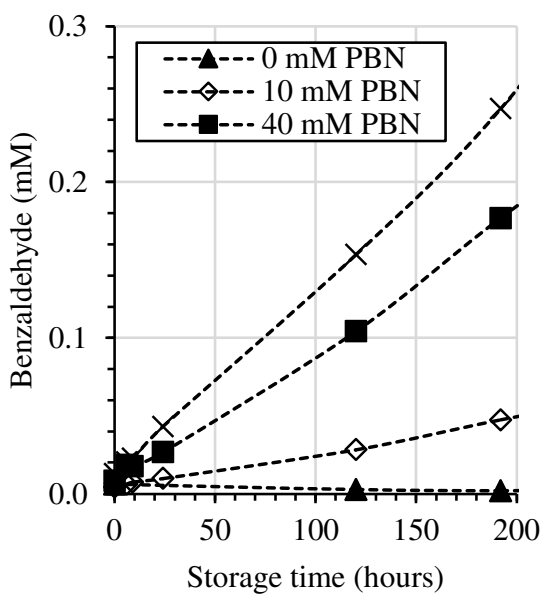

Figure 4.3. Formation of MNP-L spin adducts (A) and benzaldehyde (B) during the storage of rapeseed oil at $40{ }^{\circ} \mathrm{C}$ : free radicals were trapped with different concentrations of $\mathrm{PBN} ; \cdots \cdot \cdots 0 \mathrm{mM}, \cdots \cdots \cdots \cdot 10 \mathrm{mM}$,

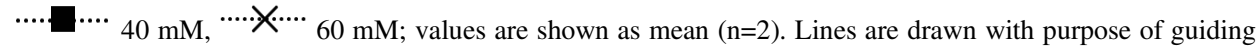
the eye.

\subsubsection{Quantitative assessment of radical formation in mayonnaise}

Next, we verified that the radical trapping and adduct degradation mechanisms observed in oil also occurred in mayonnaises. We analysed benzaldehyde generation and PBN consumption in an accelerated storage trial of mayonnaise $\left(50^{\circ} \mathrm{C}, 200\right.$ hours $)$ with ${ }^{1} \mathrm{H}$ NMR. In mayonnaise with $20 \mathrm{mM}$ PBN, the consumption of PBN was accompanied by generation of an equivalent amount of benzaldehyde (Figure S4.5). Furthermore, MNP-L adducts were detected with ESR (data not shown). This confirmed that also in the more complex mayonnaise system, PBN decomposes to benzaldehyde and MNP via the PBN-OOL intermediate. 
Since the general mechanism of the PBN spin-trapping has a major impact on the fate of radicals, we investigated this influence in mayonnaises prepared with and without EDTA. Without PBN, both the LOOH (Figure 4.4A) and aldehyde generation (Figure 4.4B) was higher in the absence of EDTA than in its presence. Different trends were observed when PBN was present in the mayonnaises. Firstly, PBN significantly $(\mathrm{p}<0.05)$ inhibited LOOH generation, both for $\mathrm{E}-(37 \mathrm{mM}(\mathrm{P}+)$ and $2 \mathrm{mM}(\mathrm{P}-)$ at $10 \mathrm{~d})$ and $\mathrm{E}+(12.5(\mathrm{P}+)$ and $8.3 \mathrm{mM}$ $(\mathrm{P}-)$ at $21 \mathrm{~d})$. Moreover, in the $\mathrm{E}-\mathrm{P}+$, the initial LOOH concentrations significantly $(\mathrm{p}<0.05)$ decreased instead of up in the first two days (from $1.7 \mathrm{mM}$ to $0.6 \mathrm{mM}$ in the first 48 hours, Figure S4.6). Secondly, the generation of $n$-alkanals was significantly higher $(p<0.05)$ in the presence of $\mathrm{PBN}$, for both $\mathrm{E}-(3.0 \mathrm{mM}(\mathrm{P}+)$ and $0.5 \mathrm{mM}(\mathrm{P}-)$ at $21 \mathrm{~d})$ and for the $\mathrm{E}+$ samples $(2.1 \mathrm{mM}(\mathrm{P}+)$ and $0.1 \mathrm{mM}(\mathrm{P}-)$ at $21 \mathrm{~d})$. This observation was especially surprising on E+ samples, which barely showed any n-alkanal generation in the absence of PBN (E+P), but high concentrations in its presence $(\mathrm{E}+\mathrm{P}+)$. For both $\mathrm{E}+\mathrm{P}+$ and $\mathrm{E}-\mathrm{P}+$, the $\mathrm{PBN}$ was consumed $(<1 \mathrm{mM})$ after 28 days (data not shown, measured with ${ }^{1} \mathrm{H} \mathrm{NMR}$ ).

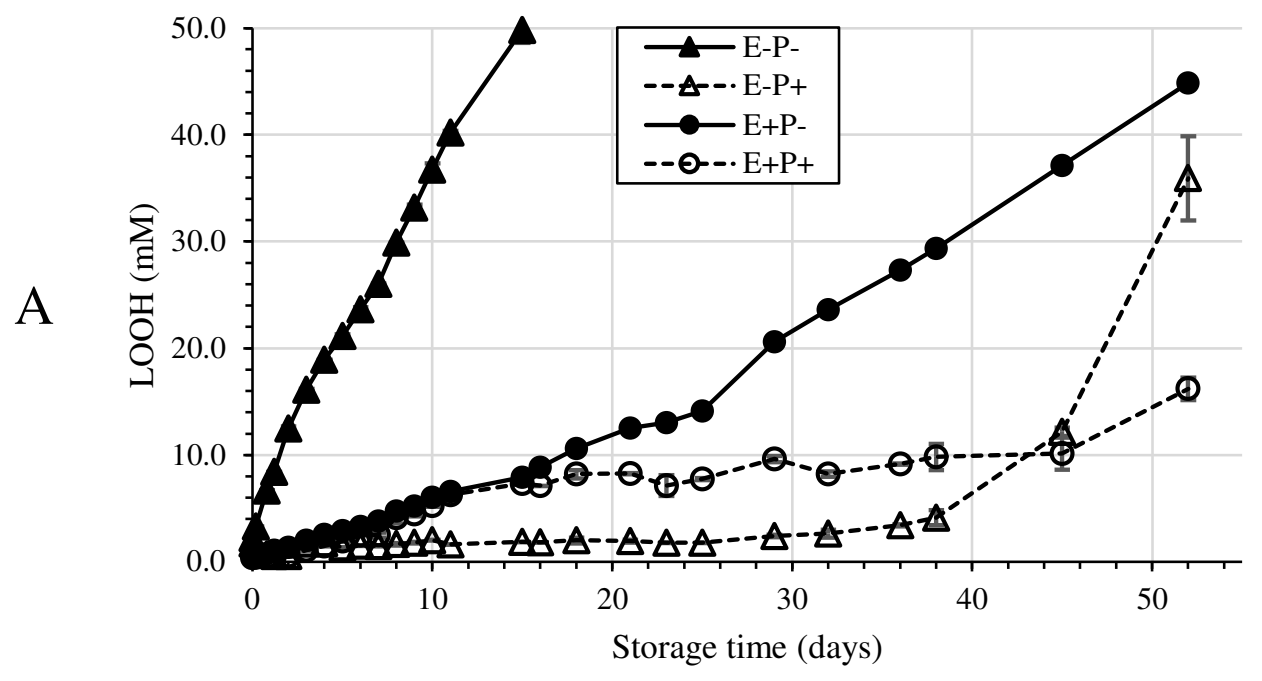



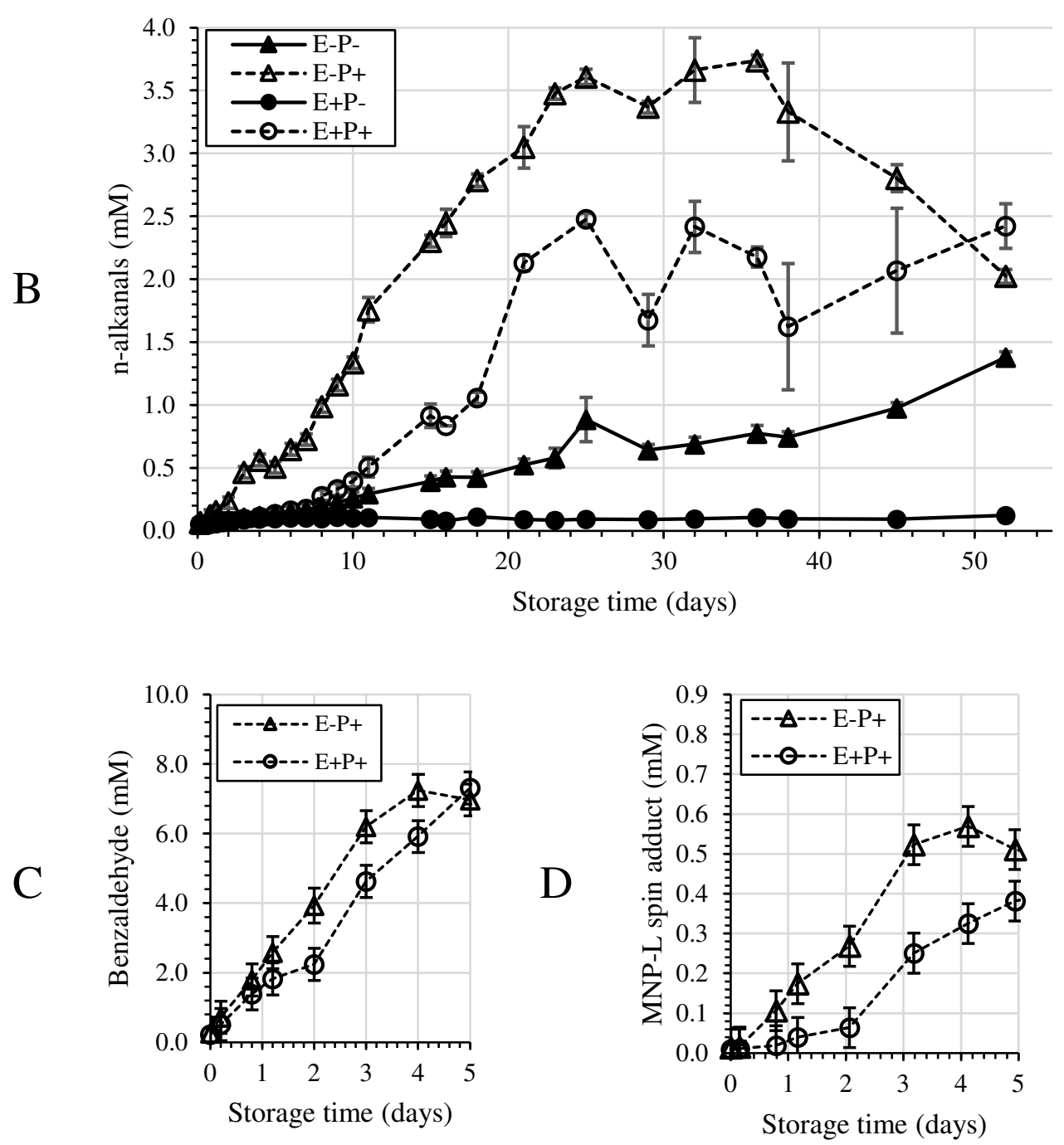

Figure 4.4. Oxidation products, $\mathrm{PBN}$ and benzaldehyde concentrations as a function of storage time at $40^{\circ} \mathrm{C}$. A: Sum of hydroperoxides by ${ }^{1} \mathrm{H}$ NMR, B: n-alkanals by ${ }^{1} \mathrm{H}$ NMR, C: benzaldehyde by ${ }^{1} \mathrm{H}$ NMR (first 5 storage days, only $\mathrm{P}+$ ), D: MNP-L by ESR (first 5 storage days only $\mathrm{P}+$ ). The investigated mayonnaise systems: with PBN (P+, open symbols), without PBN (P-, closed symbols), with EDTA (E+, circles) and without EDTA (E-, triangles). Values are shown as mean ( $\mathrm{n}=2$ for ${ }^{1} \mathrm{H}$ NMR, $\mathrm{n}=1$ for ESR (standard error $\left.0.05 \mathrm{mM}\right)$ ), with the error bars displaying the standard deviation. Lines are drawn with purpose of guiding the eye.

The difference between the $\mathrm{LOOH}$ concentrations observed in mayonnaises with and without PBN was attributed to the reactivity of PBN with LOO, effectively inhibiting the LOOHpropagation and instead generating the PBN-OOL intermediate. As discussed in section 4.3.1, the PBN-OOL intermediate is labile under present circumstances, and readily formed 
MNP, benzaldehyde and LO radicals. Both the MNP-L and benzaldehyde concentrations (Figure 4.4 C/D) were measured with ESR and NMR and increased significantly $(\mathrm{p}<0.05)$, already in the first days/hours. The LO radicals were not directly detected, but are known to undergo beta-scission, which is the main driver for the generation of aldehydes. This would explain that upon addition of PBN to mayonnaises, we observed an increase of n-alkanals, benzaldehyde and MNP-L adducts.

When EDTA was present and PBN absent (E+P-), both primary oxidation (LOOHs) and secondary oxidation (n-alkanals) products were lower than when both EDTA and PBN were absent (E-P-). Here, EDTA worked as expected as a secondary antioxidant through the chelation of iron to inhibit the catalysing effect of this transition metal. The redox $\mathrm{Fe}^{2+} / \mathrm{Fe}^{3+}$ cycling catalytic action is reported to go primarily via existing LOOHs (Laguerre et al., 2020; Merkx, Delić, Wierenga, Hennebelle, \& van Duynhoven, 2019; Mozuraityte, Rustad, \& Storro, 2008). These LOOHs could decompose to form LOO and LO radicals, which will respectively fuel the propagation of $\mathrm{LOOH}$ generation and form aldehydes via beta-scission of LO (Figure 4.5). In the presence of PBN, the LOO- radicals can react with both PBN and $\mathrm{LH}$ to form respectively the LOO-PBN intermediates and LOOHs. In the presence of both PBN and EDTA $(\mathrm{E}+\mathrm{P}+)$, there is little to no free iron (or any other transition metal) in the mixture to catalyse the decomposition of $\mathrm{LOOH}$. Therefore, the accumulated LOOHs will not degrade, which results in a steady increase of LOOHs. However, in the absence of EDTA (E-P+), free transition metals will be present and catalyse the decomposition of LOOHs to $\mathrm{LOO} \cdot$ and $\mathrm{LO} \cdot$ radicals. This means that for $\mathrm{E}-\mathrm{P}+$, contrary to $\mathrm{E}+\mathrm{P}+, \mathrm{LOOH}$ will be degraded much more rapidly, which explains the initial decrease of $\mathrm{LOOH}$ in $\mathrm{E}-\mathrm{P}+$ and why the $\mathrm{LOOH}$ levels are very low until the moment PBN was consumed ( 28 days). Furthermore, the first 28 days, the $n$-alkanal levels (a beta-scission product of LO·) are significantly $(\mathrm{p}<0.05$ ) higher for the $\mathrm{E}-\mathrm{P}+$ than the $\mathrm{E}+\mathrm{P}+$. 


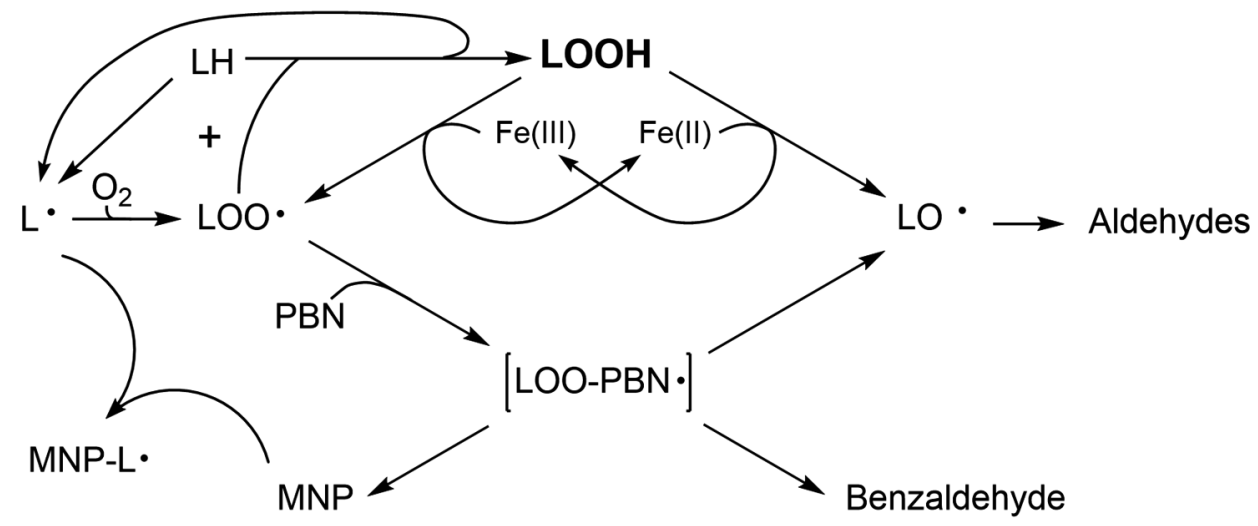

Figure 4.5. Schematic overview of modulation of lipid oxidation pathways in mayonnaise by PBN spin-trapping.

\subsubsection{Early assessment of antioxidant protection against primary oxidation in mayonnaise}

PBN spin trapping has a significant impact on lipid oxidation mechanisms, but whether PBN could be used as an early oxidative assessment tool was still undisclosed. In the experiment discussed in 4.3.2, the MNP-L concentration started to decrease after eight days and was no longer detectable by ESR after 50 days (Figure S4.6). In the first five days, MNP-L concentrations were already more than ten times lower than the benzaldehyde concentrations (Figure $4.4 \mathrm{C} / \mathrm{D}$ ), which hinted at a considerable MNP-L reduction in mayonnaises compared to oil (section 4.3.1). Nonetheless, MNP-L formation (Figure 4.4 D) was clearly delayed in the presence of EDTA $(\mathrm{E}+\mathrm{P}+)$ compared to the mayonnaise without EDTA $(\mathrm{E}-\mathrm{P}+)$. This suggested that MNP-L generation can be used as an early indicator for lipid oxidation in mayonnaise.

The applicability of PBN-spin trapping for early assessment of oxidative stability lipid oxidation in mayonnaises was verified in a trial with mayonnaises with four different levels of EDTA stored at $50{ }^{\circ} \mathrm{C}$ with a PBN concentration of $10 \mathrm{mM}$. Increasing EDTA concentrations were associated with a decrease in the formation of MNP-L adducts, as measured with ESR. Within five hours, the different formulations could be clearly significantly $(\mathrm{p}<0.05)$ ranked based on their MNP-L levels (Figure 4.6A)). Lowering the PBN concentration (i.e. from $10 \mathrm{mM}$ to $1 \mathrm{mM}$ ) might have a less perturbing effect on the oxidative mechanism, with the drawback of generation less MNP-L in the process and was therefore not investigated at this point. The distinctive power of ESR was matched. Such a distinction between the formulations were possible only after 4 days when considering the inhibition of LOOH formation measured by ${ }^{1} \mathrm{H}$ NMR. Moreover, at room temperature, MNP- 
L assessment by ESR allowed oxidative ranking after five days (Figure S4.7, no ${ }^{1} \mathrm{H}$ NMR data). Thus, although PBN addition significantly altered the oxidative mechanism, it has the potential to be used as a tool to rank the oxidative stability of emulsions via the determination of the MNP-L adducts.

A

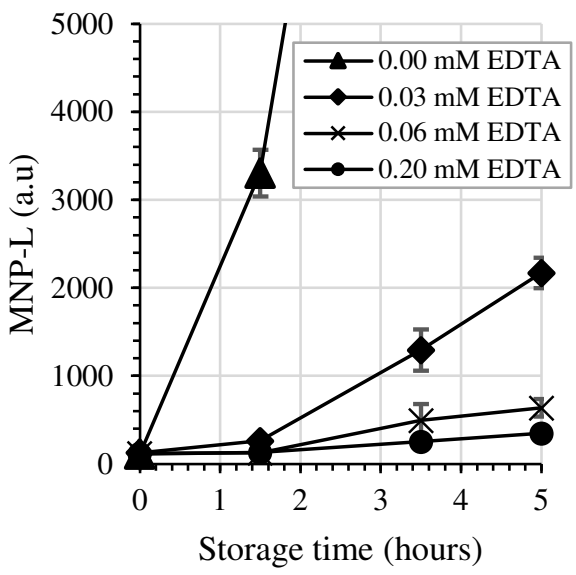

$\mathrm{B}$

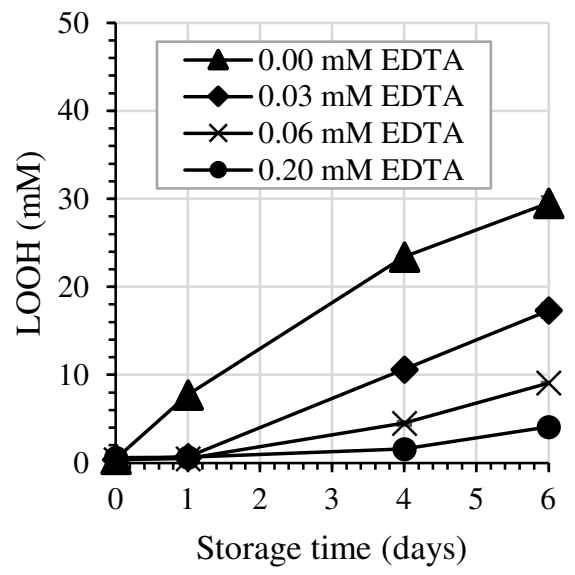

Figure 4.6. MNP-L and hydroperoxides in mayonnaises as a function of different storage times at $50{ }^{\circ} \mathrm{C}$. A: relative MNP-L concentration in the presence of $10 \mathrm{mM}$ PBN, B: absolute LOOH concentrations in the absence of PBN. The four lines represent: 0 mM EDTA ( $\neg$ ), $0.03 \mathrm{mM}$ EDTA $(\neg), 0.06 \mathrm{mM}$ EDTA $(\boldsymbol{\bullet}$ ) and $0.2 \mathrm{mM}$ EDTA $(\longrightarrow)$ ).

\subsection{Conclusion}

In mayonnaise, already at mild $\left(=<50^{\circ} \mathrm{C}\right)$ shelf-life conditions, the PBN-OOL adducts rapidly degrades to MNP, benzaldehyde and alkoxy-radicals. The observed ESR-signal predominantly originates from the adducts of MNP and alkyl radicals. Benzaldehyde concentrations can be determined by ${ }^{1} \mathrm{H}$ NMR and used for estimating the cumulative amount of LOO that has been trapped by PBN. Hence, both NMR and ESR can be used in combination with PBN to assess LOO formation in early (hours-days) stages of lipid oxidation. The trapping of LOO radicals and subsequent generation of LO radicals impacts downstream reaction pathways, which can hinder mechanistic studies in the presence of PBN. PBN spin-trapping in combination with ESR and NMR detection does allow for assessment of antioxidant protection against primary oxidation at mild conditions. 


\subsection{Literature}

Andersen, M. L., \& Skibsted, L. H. (2006). ESR Spectroscopy for the Study of Oxidative Processes in Food and Beverages. In G. A. Webb (Ed.), Modern Magnetic Resonance (pp. 1861-1866). Dordrecht: Springer Netherlands.

Barriuso, B., Astiasarán, I., \& Ansorena, D. (2013). A review of analytical methods measuring lipid oxidation status in foods: a challenging task. European Food Research and Technology, 236(1), 1-15.

Berton-Carabin, C. C., Ropers, M.-H., \& Genot, C. (2014). Lipid Oxidation in Oil-in-Water Emulsions: Involvement of the Interfacial Layer. Comprehensive Reviews in Food Science and Food Safety, 13(5), 945-977.

Berton, C., Ropers, M.-H., Viau, M., \& Genot, C. (2011). Contribution of the Interfacial Layer to the Protection of Emulsified Lipids against Oxidation. Journal of Agricultural and Food Chemistry, 59(9), 5052-5061.

Boerekamp, D. M. W., Andersen, M. L., Jacobsen, C., Chronakis, I. S., \& García-Moreno, P. J. (2019). Oxygen permeability and oxidative stability of fish oil-loaded electrosprayed capsules measured by Electron Spin Resonance: Effect of dextran and glucose syrup as main encapsulating materials. Food Chemistry, 287, 287-294.

Cui, L., Lahti, P. M., \& Decker, E. A. (2016). Evaluating Electron Paramagnetic Resonance (EPR) to Measure Lipid Oxidation Lag Phase for Shelf-Life Determination of Oils. Journal of the American Oil Chemists' Society, 94(1), 89-97.

Frankel, E. N. (2005). Free radical oxidation. In Lipid Oxidation (2nd ed., pp. 15-24): Elsevier.

Janzen, E. G., Krygsman, P. H., Lindsay, D. A., \& Haire, D. L. (1990). Detection of Alkyl, Alkoxyl, and Alkyperoxyl Radicals from the Thermolysis of Azobis(isobutyronitrile) by ESR/Spin Trapping. Evidence for Double Spin Adducts from Liquid-Phase Chromatography and Mass Spectroscopy. Journal of American Chemical Society, 112, 8278-8284.

Laguerre, M., Tenon, M., Bily, A., \& Birtić, S. (2020). Toward a Spatiotemporal Model of Oxidation in Lipid Dispersions: A Hypothesis-Driven Review. European Journal of Lipid Science and Technology, n/a(n/a), 1900209.

Merkx, D. W. H., Delić, F., Wierenga, P. A., Hennebelle, M., \& van Duynhoven, J. P. M. (2019). 31P NMR assessment of the phosvitin-iron complex in mayonnaise. Magnetic Resonance in Chemistry, 57(9), 540-547.

Merkx, D. W. H., Hong, S., Ermacora, A., \& van Duynhoven, J. (2018). Rapid Quantitative Profiling of Lipid Oxidation Products in a Food Emulsion by 1H NMR. Analytical Chemistry, 90(7), 4863-4870.

Mozuraityte, R., Rustad, T., \& Storro, I. (2008). The Role of Iron in Peroxidation of Polyunsaturated Fatty Acids in Liposomes. Journal of Agricultural and Food Chemistry, 56, 537-543.

Schaich, K. M. (2012). Thinking outside the classical chain reaction box of lipid oxidation. Lipid Technology, 24(3), 55-58.

Schaich, K. M., Xie, J., \& Bogusz, B. A. (2017). Thinking outside the classical chain reaction box of lipid oxidation: Evidence for alternate pathways and the importance of epoxides. Lipid Technology, 29(9-10), 91-96.

Silvagni, A., Franco, L., Bagno, A., \& Rastrelli, F. (2010). Thermoinduced Lipid Oxidation of a Culinary Oil: A Kinetic Study of the Oxidation Products by Magnetic Resonance Spectroscopies. J. Phys. Chem. A, 114, 10059-10065. 
Skiera, C., Steliopoulos, P., Kuballa, T., Holzgrabe, U., \& Diehl, B. (2012). 1H NMR approach as an alternative to the classical p-anisidine value method. European Food Research and Technology, 235, 1101-1105.

Steenhorst-Slikkerveer, L., Louter, A., Janssen, H.-G., \& Bauer-Plank, C. (2000). Analysis of nonvolatile lipid oxidation products in vegetable oils by normal-phase highperformance liquid chromatography with mass spectrometric detection. Journal of the American Oil Chemists' Society, 77(8), 837.

Stoll, S., \& Schweiger, A. (2006). EasySpin, a comprehensive software package for spectral simulation and analysis in EPR. Journal of Magnetic Resonance, 178(1), 42-55.

Thomsen, M. K., Jacobsen, C., \& Skibsted, L. H. (2000). Mechanism of initiation of oxidation in mayonnaise enriched with fish oil as studied by electron spin resonance spectroscopy. Eur Food Res Technol, 211, 381-386.

Thomsen, M. K., Kristensen, D., \& Skibsted, L. H. (2000). Electron Spin Resonance Spectroscopy for Determination of the Oxidative Stability of Food Lipids. Journal of American Oil Chemical Society, 77(7), 725-730.

Thomsen, M. K., Vedstesen, H., \& Skibsted, L. H. (1999). Quantification of Radical Formation in Oil-in-Water Food Emulsions by Electron Spin Resonance Spectroscopy. Journal of Food Lipids, 6, 149-158.

Velasco, J., Andersen, M. L., \& Skibsted, L. H. (2004). Evaluation of oxidative stability of vegetable oils by monitoring the tendency to radical formation. A comparison of electron spin resonance spectroscopy with the Rancimat method and differential scanning calorimetry. Food Chemistry, 85(4), 623-632.

Velasco, J., Andersen, M. L., \& Skibsted, L. H. (2005). Electron Spin Resonance Spin Trapping for Analysis of Lipid Oxidation in Oils: Inhibiting Effect of the Spin Trap alpha-Phenyl-N-tert-butylnitrone on Lipid Oxidation. Journal of Agricultural and Food Chemistry, 53, 1328-1336.

Vicente, M. L., Empis, J. A., Deighton, N., Glidewell, S. M., Goodman, B. A., \& Rowlands, C. C. (1998). Use of EPR and ENDOR spectroscopy in conjunction with the spin trapping technique to study the high-temperature oxidative degradation of fatty acid methyl esters Journal of the Chemical Society,, Perkin Transactions 2, 449-454. 


\subsection{Supporting information}

\section{S4.1 DMSO vs MCT Oil}

The solubility of PBN in DMSO is higher than in MCT oil. In this study, we used both PBN in DMSO and PBN in MCT oil solutions. To verify whether they could be used interchangeably, $20 \mathrm{mM}$ PBN was dissolved in both solvents and then added to mayonnaise. We tracked both the generation of spin adducts with ESR and degradation of PBN with NMR. No notable difference in the spin adduct formation or in PBN degradation was observable between the two tested solvents MCT and DMSO.

A

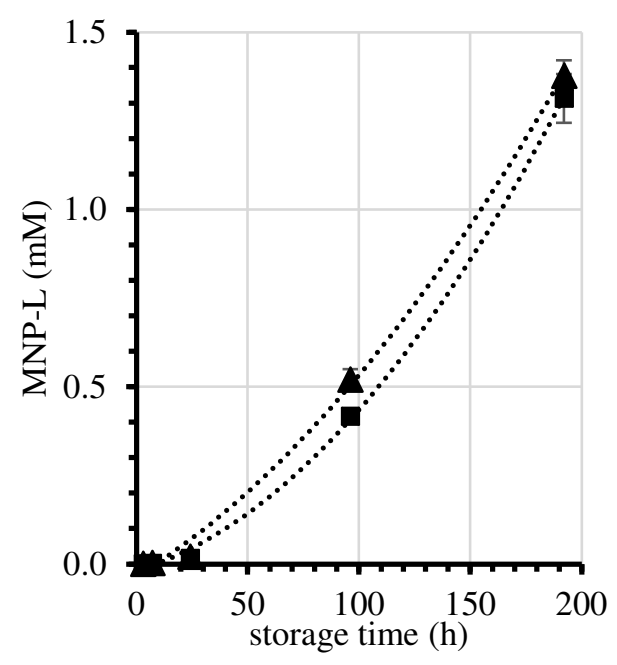

B

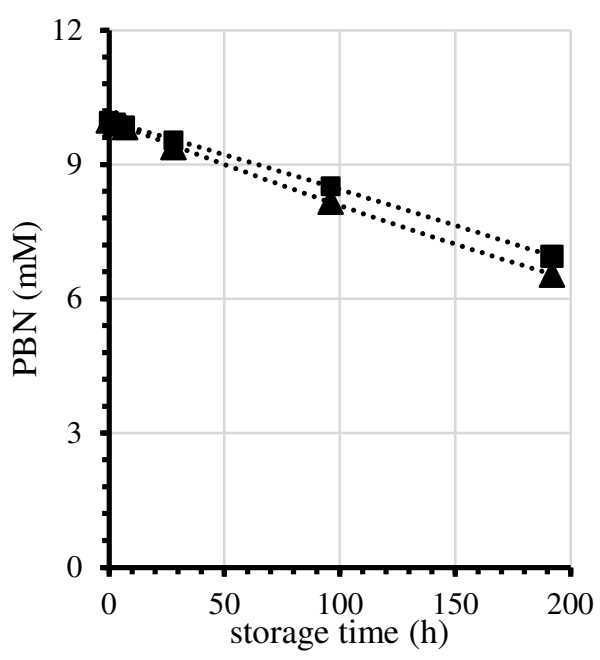

Figure S4.1. Formation of MNP-L adducts and decrease of PBN during the storage of mayonnaise at $40{ }^{\circ} \mathrm{C}$. Different solvents were used to dissolve PBN: - MCT oil $\Delta$ DMSO; A) MNP-L adducts by ESR, B) PBN concentrations by ${ }^{1} \mathrm{H}$ NMR. Values are shown as mean $\pm \mathrm{SD}(\mathrm{n}=3)$. Lines are drawn with purpose of guiding the eye. 


\section{S4.2 MNP in oil vs PBN in mayo}

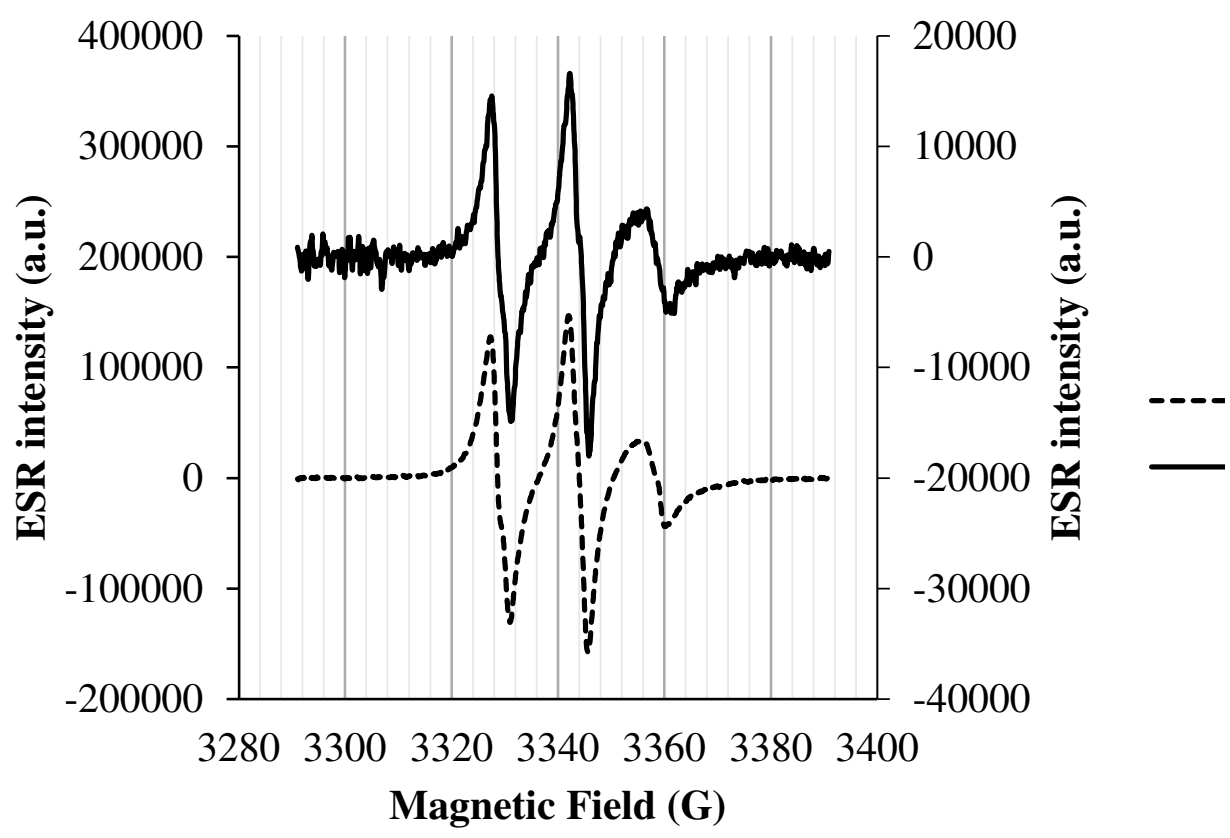

Figure S4.2. Visual comparison of the ESR spectra generated under mild storage conditions $\left(40{ }^{\circ} \mathrm{C}\right)$ in oil with MNP as spin trap (---) and in mayonnaise with PBN as spin trap (-). 


\section{S4.3 benzaldehyde and PBN assignment}<smiles>[2H]c1cccc(/C=[N+](\[O-])C(C)(C)C)c1C</smiles>

b

PBN<smiles>[2H]c1ccccc1</smiles>

3

Benzaldehyde
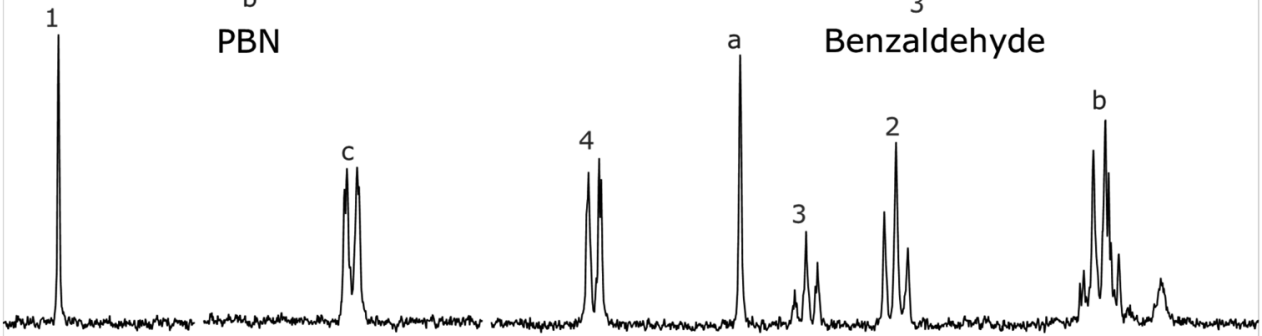

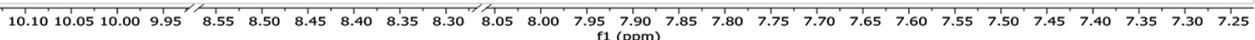

Figure S4.3. Aromatic region of the ${ }^{1} \mathrm{H}$ NMR spectrum of the oil layer of mayonnaise with $20 \mathrm{mM}$ PBN stored at $40{ }^{\circ} \mathrm{C}$ for 12 days. The spectrum is recorded in deuterated acetone. The (purple) numbers in the spectral assignment correspond to benzaldehyde, the (red) letters to PBN. 
S4.4 MNP-L and benzaldehyde quantification in the oxygen variation studies.
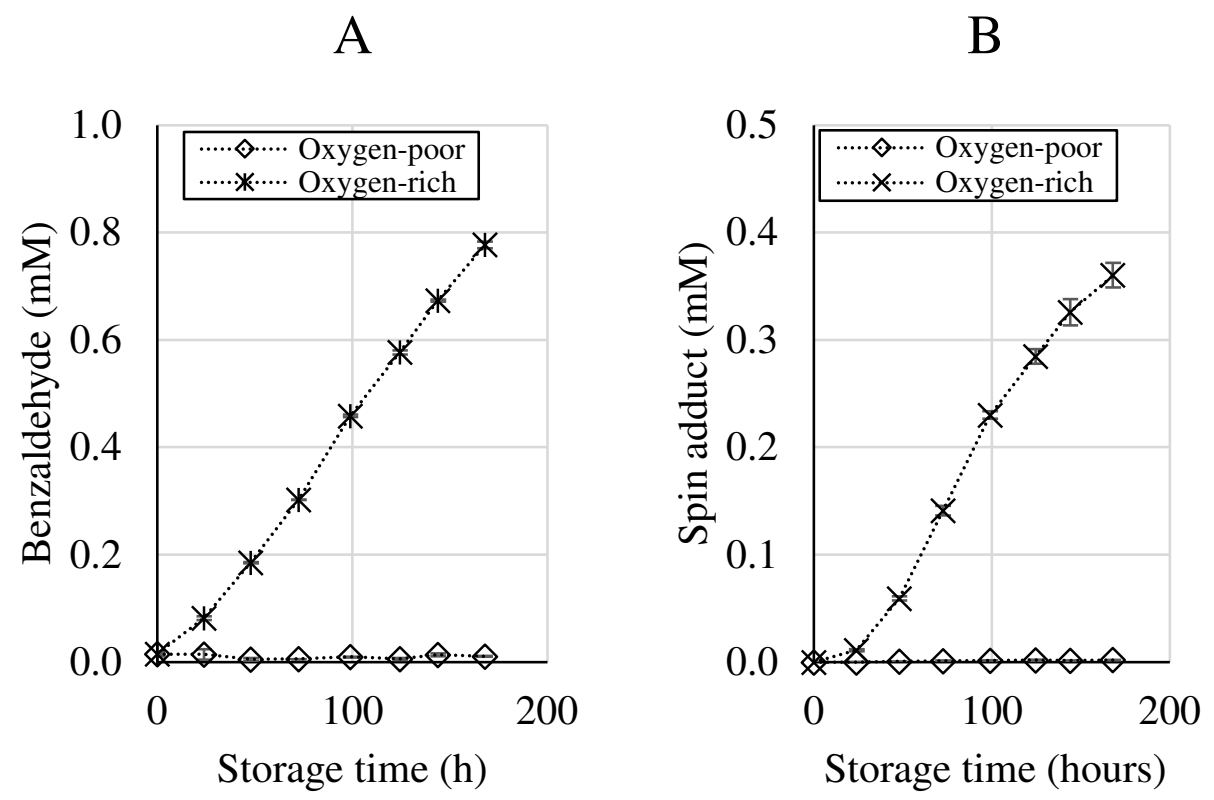

Figure S4.4. Measured benzaldehyde (A) and spin adducts (B) during the storage of rapeseed oil at $50{ }^{\circ} \mathrm{C}$ under different oxygen conditions: $\cdots \times \cdots \cdots$ oxygen rich (spin-adduct is MNP-L), $\cdots \odot \cdots$ oxygen depleted (spinadduct is $\mathrm{PBN}-\mathrm{L})$; values are shown as mean $\pm \mathrm{SD}(\mathrm{n}=2)$. Lines are drawn with purpose of guiding the eye. 
S4.5 Benzaldehyde increase and PBN decrease in mayonnaise.

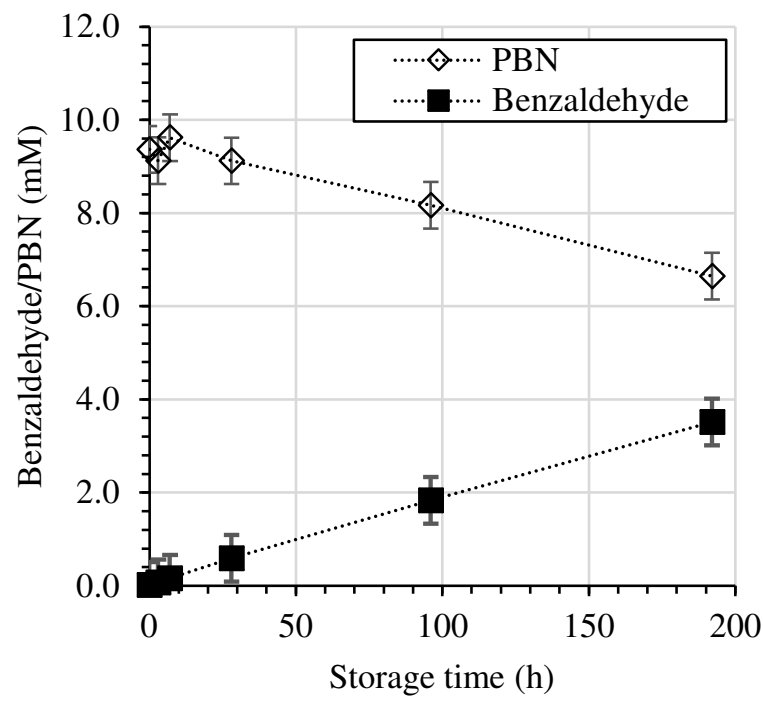

Figure S4.5. Increase of benzaldehyde $(\cdots \cdot \cdots \cdot \cdots)$ and decrease of PBN $(\cdots \bullet \cdot \cdots)$ during the storage of mayonnaise at $50{ }^{\circ} \mathrm{C}$, quantified by ${ }^{1} \mathrm{H}$ NMR. Lines are drawn with purpose of guiding the eye. 
S4.6 Full curves on the LOOH/Benzaldehyde/MNP-L curves in Figure 4.6.
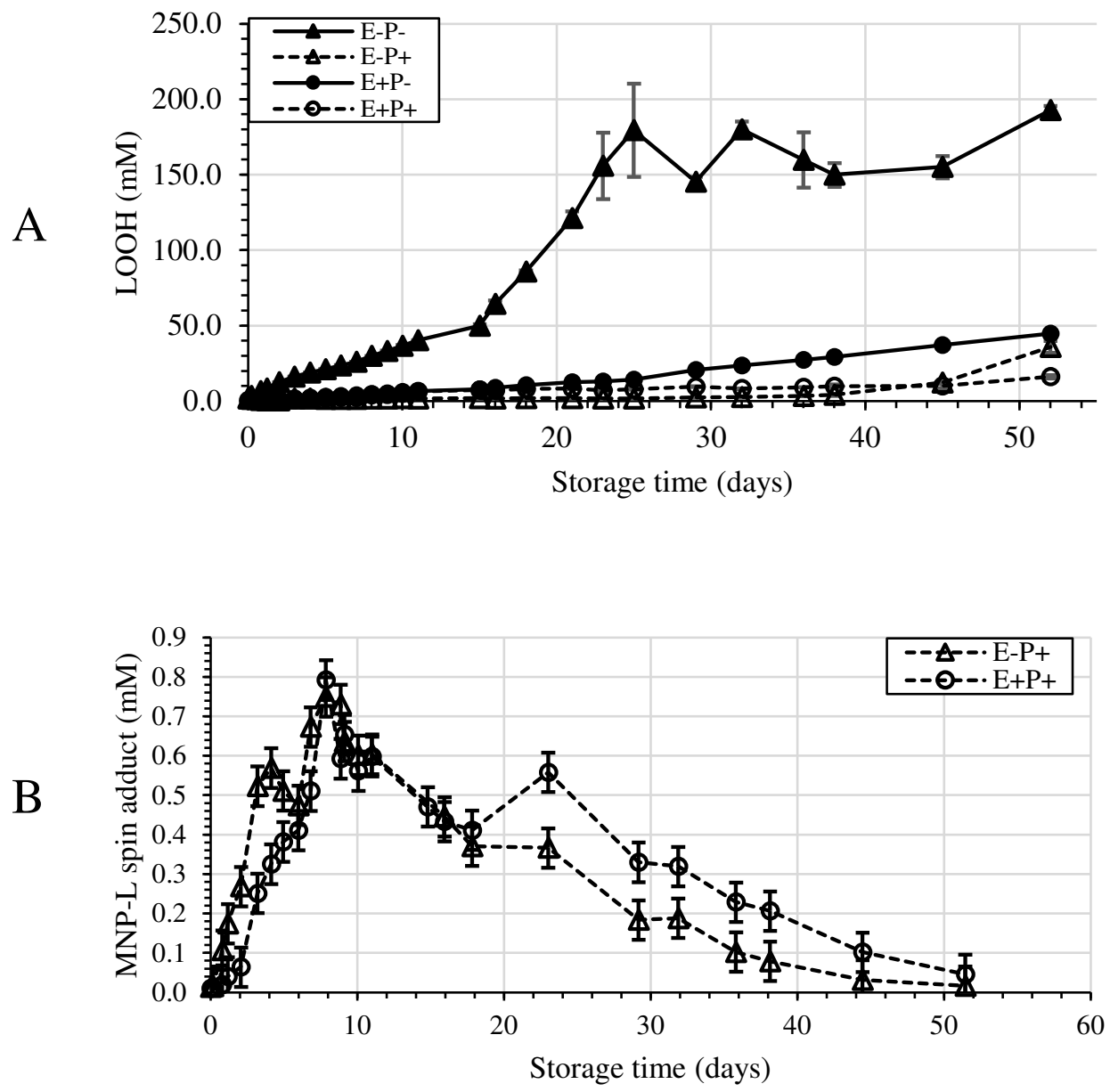


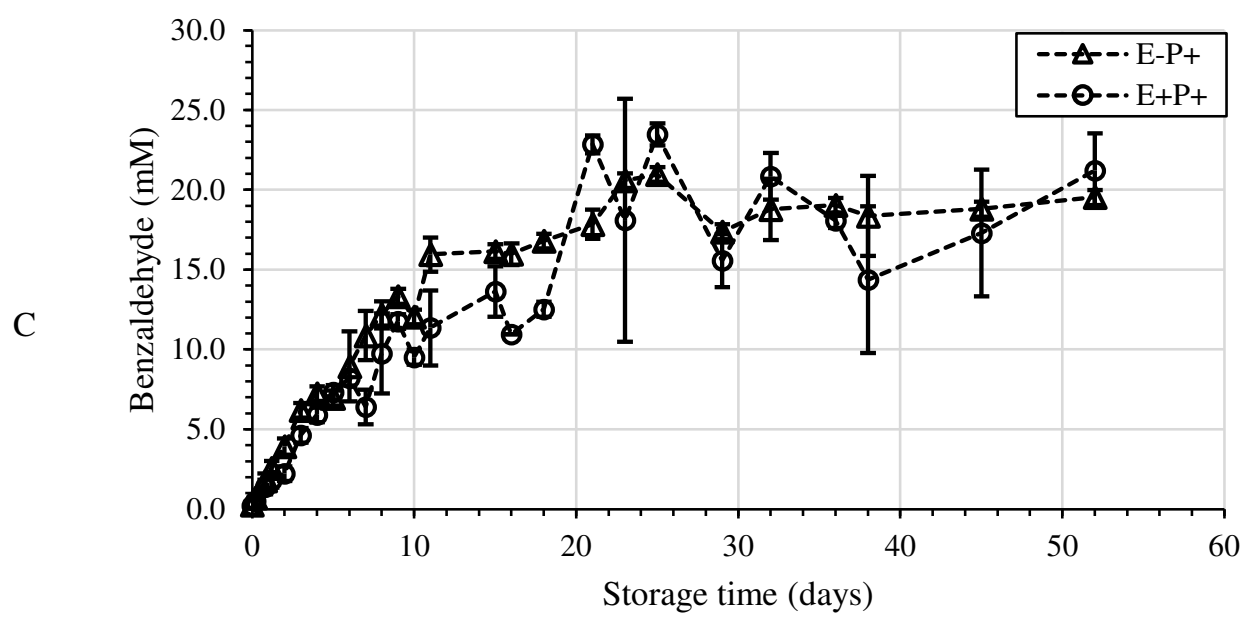

Figure S4.6. $\mathrm{LOOH}, \mathrm{PBN}$ and benzaldehyde concentrations as a function of storage time at $40{ }^{\circ} \mathrm{C}$. A: Sum of hydroperoxides by ${ }^{1} \mathrm{H}$ NMR, B: MNP-L by ESR, C: benzaldehyde by ${ }^{1} \mathrm{H}$ NMR. The four systems that were explored: mayonnaise with 75 ppm EDTA and $20 \mathrm{mM} \mathrm{PBN}(\mathrm{E}+\mathrm{P}+,-\bullet-\bullet)$, without EDTA and with $20 \mathrm{mM}$ PBN (E-P+, - $-\boldsymbol{\Delta}-\boldsymbol{*})$, with 75 ppm EDTA and without PBN (E+P-, - ) and without both EDTA and PBN (E-P-, Values are shown as mean ( $\mathrm{n}=2$ for ${ }^{1} \mathrm{H}$ NMR, $\mathrm{n}=1$ for ESR (standard error $\left.0.05 \mathrm{mM}\right)$ ), with the error bars displaying the standard deviation. Lines are drawn with purpose of guiding the eye. 


\section{S4.7 Oxidative assessment under ambient storage conditions.}

In parallel to the samples described in 4.3.3 and 4.2.3, the same experiment was done, but then at lower storage temperatures. The mixtures were incubated at ambient temperature (20 $-22^{\circ} \mathrm{C}$ ) and aliquots were collected at every day until 7 days and stored at $-80{ }^{\circ} \mathrm{C}$ until further analysis.

A

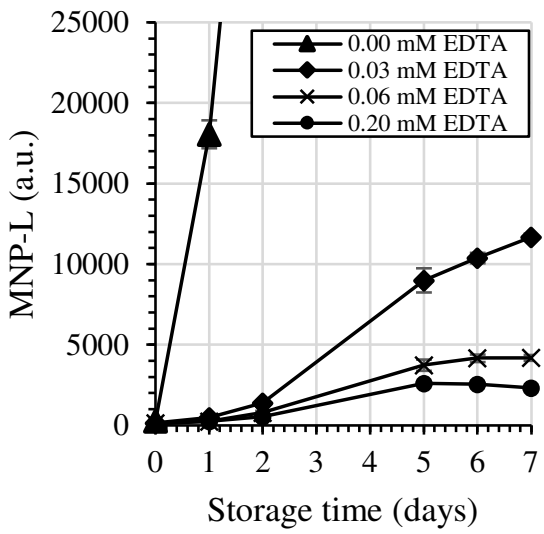

B

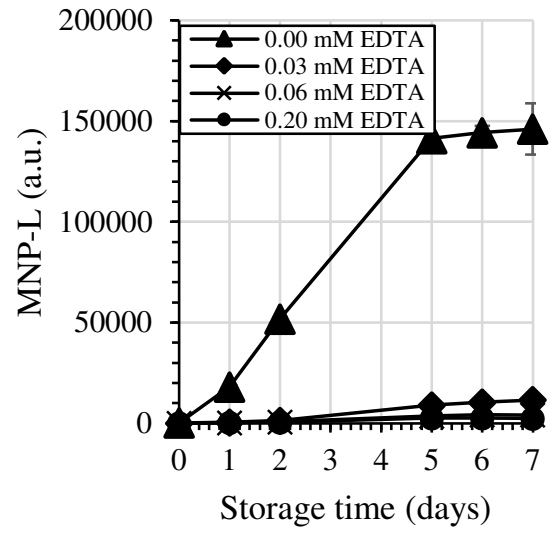

Figure S4.7. MNP-L as a function of storage time at $20-22{ }^{\circ} \mathrm{C}$. A/B: relative MNP-L concentration in the presence of $10 \mathrm{mM}$ PBN with different scales on the y-axis. The four lines represent: $0.00 \mathrm{mM}$ EDTA ( - ),

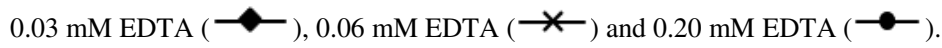




$$
5
$$




\section{Chapter 5}

\section{Quantitative and predictive modelling of lipid oxidation in mayonnaise}

Based on: Merkx, D. W. H., Swager, A., van Velzen, E. J. J., van Duynhoven, J. P. M., \& Hennebelle, M. (2021). Quantitative and Predictive Modelling of Lipid Oxidation in Mayonnaise. Antioxidants, 10(2), 287. 


\begin{abstract}
Food emulsions with high amounts of unsaturated fats, such as mayonnaise, are prone to lipid oxidation. In the food industry, typically accelerated shelf-life tests are applied to assess the oxidative stability of different formulations. Here, the appearance of aldehydes at the socalled onset time, typically weeks, is considered a measure for oxidative stability of food emulsions, such as mayonnaise. To enable earlier assessment of compromised shelf-life a predictive model for volatile off-flavour generation was developed. The model was based on formation kinetics of hydroperoxides, which are early oxidation products and precursors of volatile aldehydes, responsible for off-flavour. Under accelerated shelf-life conditions (50 ${ }^{\circ} \mathrm{C}$ ), hydroperoxide (LOOH) concentration over time shows a sigmoidal curvature followed by an acceleration phase that occurs at a $\mathrm{LOOH}$-concentration between $38-50 \mathrm{mmol} / \mathrm{kg}$, here interpreted as a critical $\mathrm{LOOH}$ concentration $\left(\mathrm{CC}_{\mathrm{LOOH}}\right)$. We hypothesised that the time at which $\mathrm{CC}_{\mathrm{LOOH}}$ was reached was related to the onset of aldehyde generation and the characterisation of the LOOH-generation curvature could be based on reaction kinetics in the first days. These hypotheses were tested using semi-empirical models to describe the autocatalytic character of hydroperoxide formation in combination with the $\mathrm{CC}_{\mathrm{LOOH}}$. The Foubert function was selected as best describing the $\mathrm{LOOH}$-curvature and was hence used to accurately predict onset of aldehyde generation, in most cases within several days of shelflife. Furthermore, we found that defining parameters of this model could be used to recognise antioxidant mechanisms at play.
\end{abstract}




\subsection{Introduction}

In high-fat food emulsions such as mayonnaise, lipid oxidation is a major concern due to the high amount of unsaturated lipids that can undergo oxidation reactions. The first step is a free radical reaction that yields hydroperoxides (LOOHs), a meta-stable product that can degrade further into aldehydes, epoxides and other secondary oxidation products. Antioxidants (AOs) can be used to combat this process, heavily depending on the type of the oil matrix, where, for example fish oil (Jacobsen, Sørensen, \& Nielsen, 2013), oat flakes (Zbikowska et al., 2018) and mayonnaises (Schröder, Sprakel, Boerkamp, Schroën, \& Berton-Carabin, 2019; Uluata, McClements, \& Decker, 2015) all have different optimal AO systems. To assess these AOs and thus the oxidative stability of high-fat food emulsions, which under consumer relevant conditions are stable for more than a few months, the food industry applies accelerated shelf-life tests. Here the oxidation reactions are stimulated to enable faster assessment (Calligaris, Manzocco, Anese, \& Nicoli, 2016; Manzocco, Calligaris, Anese, \& Nicoli, 2016), which can be achieved in several ways, such as the addition of pro-oxidants e.g. transition metals (Berton, Ropers, Bertrand, Viau, \& Genot, 2012; Gutteridge, Richmond, \& Halliwell, 1979; Schröder et al., 2019), manipulation of oxygen content and state (Johnson \& Decker, 2015) or most commonly the increase of storage temperature (Gómez-Alonso, Mancebo-Campos, Desamparados Salvador, \& Fregapane, 2004; Ragnarsson \& Labuza, 1977). In these enhanced storage tests, different markers can be measured over time. One of the most frequently monitored markers is hexanal, a secondary lipid oxidation product that is linked to perceived off-taste and off-flavour of the product. However, even under accelerated conditions, hexanal assessment still requires several (4-8) weeks to be assessed accurately. An earlier marker are the hydroperoxides, being the first stable oxidation product to be formed, these can be detected from the first days onward. However, not these hydroperoxides, but the aldehydes are responsible for the off-taste and smell of the final product. To this end, it would be of great value to quantitatively predict the onset of aldehyde generation using initial hydroperoxide generation.

In this work, lipid oxidation in food emulsions is accelerated by storage at elevated temperature $\left(50{ }^{\circ} \mathrm{C}\right)$, where literature suggests that inter-droplet transport and diffusion mechanisms are not rate limiting (Budilarto \& Kamal-Eldin, 2015; Laguerre, Tenon, Bily, \& Birtic, 2020). We hypothesised that the main mechanisms at play are the known free radical mediated reactions and we also attribute a colloidal role to reverse micelle formation, which has been shown to play a part in lipid oxidation kinetics (Bingcan Chen, Han, Laguerre, McClements, \& Decker, 2011; B. Chen, Panya, McClements, \& Decker, 2012). First, these free radical reactions are autocatalytic reactions that can be observed as sigmoidal trends (Pinchuk \& Lichtenberg, 2002, 2014; Velasco, Andersen, \& Skibsted, 2005). In this 
autocatalytic reaction, the initial phase can be hampered by the presence of primary antioxidants or the general lack of initiators. This is followed by a sudden increase of oxidation products being formed due to the ample presence of the unsaturated double bonds and oxygen. The generation of hydroperoxides will then decelerate due to the consumption of oxygen, the decrease of oxidizable substrate and the degradation of the hydroperoxides, resulting in a sigmoidal time-response curves. Sigmoidal time-response curves can be described mathematically by exponential functions with three or four parameters, as described in Gompertz (1825) and Foubert, Dewettinck, and Vanrolleghem (2003). Secondly, reverse micelles might play a critical role in the propagation of lipid oxidation products as they would be able to capture pro-oxidant water soluble compounds and transport them throughout the lipid layer (Chaiyasit, Elias, McClements, \& Decker, 2007; Chaiyasit, McClements, Weiss, \& Decker, 2008). Micellization is reported to be stimulated even more by the accumulation of lipid oxidation products, specifically the hydroperoxides (Brimberg \& Kamal-Eldin, 2003). In other words, after a critical concentration of hydroperoxides $\left(\mathrm{CC}_{\mathrm{LOOH}}\right)$ formed at an oxidizable interface, these hydroperoxides will stabilise reverse micelles and promote further oxidation inside the droplet. We hypothesise that when the $\mathrm{CC}_{\mathrm{LOOH}}$ is reached, both primary and secondary oxidation mechanisms are enhanced significantly, making the rapid acceleration of aldehydes imminent. In recent years, new methods, utilizing Liquid Chromatography - Mass Spectrometry (LC-MS) and Electron Spin Resonance (ESR) (Hollebrands, Varvaki, Kaal, \& Janssen, 2018; Merkx et al., 2021) have been developed to complement the existing methods, such as hexanal by Gas Chromatography (GC) and Peroxide Value (PV) (Frankel, Hu, \& Tappel, 1989; "ISO 3960:2017," 2017) that allow fast and quantitative measurements of the key primary and secondary oxidation markers. Moreover, proton Nuclear Magnetic Resonance ( ${ }^{1} \mathrm{H}$ NMR) methods have been developed that provide a rapid and accurate quantification of the bulk of hydroperoxides and (non-volatile) aldehydes (Merkx, Hong, Ermacora, \& van Duynhoven, 2018). The simultaneous quantification of both primary and secondary lipid oxidation products raises new opportunities to gain more mechanistical insight on the process and to develop quantitative predictive modelling approaches.

Here, we assessed which of the sigmoidal Gompertz and Foubert functions best described best hydroperoxide generation (measured by ${ }^{1} \mathrm{H}$ NMR) in mayonnaise in accelerated shelflife tests. Next, the selected function was used to predict the aldehyde onset, based on the hypothesis that reaching the $\mathrm{CC}_{\mathrm{LOOH}}$ is a trigger for accelerated secondary oxidation. This work was initially done on a wide set of data collected throughout the years, with a wide variety of ingredients and formulations. Afterwards, we validated this semi-empirical prediction model, using a designed experiment which included antioxidants with known mechanisms. The fitted model parameters were interpreted in mechanistic terms. 


\subsection{Material and Methods}

\subsubsection{Materials}

CDCl3, DMSO-d6 and 4 $\mathrm{A}$ molsieves were purchased from Euriso-top (Saint-Aubin, France). Rapeseed (RP) oil, egg yolk, egg blend, sodium chloride and spirit vinegar were purchased from local suppliers. Ethylenediaminetetraacetic acid calcium disodium salt ( $\left.\mathrm{CaNa}_{2} \mathrm{EDTA}\right)$ and gallic acid were purchased from Sigma Aldrich (Zwijndrecht, the Netherlands).

\subsubsection{Sample preparation}

For the model selection and initial prediction content and a wide range of natural and chemical antioxidants in different concentrations (more details in section 2.4 and Table S1). Emulsification was done using emulsification with a Silverson mixer (Silverson, USA) for 6 minutes at $8.500 \mathrm{rpm}$. The water phase (final concentration $22-35 \% \mathrm{w} / \mathrm{w}$ ) consisted mainly of egg yolk $(\sim 5 \% \mathrm{w} / \mathrm{w})$ or egg blend $(\sim 8 \% \mathrm{w} / \mathrm{w})$ and $\mathrm{NaCl}(1-1.5 \% \mathrm{w} / \mathrm{w})$. Prior to the emulsification, antioxidants (if dosed) were added to the water phase. Next, rapeseed oil (65$78 \% \mathrm{w} / \mathrm{w})$ was slowly added to form the emulsion and finally spirit vinegar $(1.4 \% \mathrm{w} / \mathrm{w})$ to achieve the desired $\mathrm{pH}$ (3.8). In the validation study (sections 3.4 and 3.5), mayonnaises were prepared with egg yolk (5\%w/w) and rapeseed oil (78\%). EthyleneDiamineTetraAcetic acid (EDTA/E) and Gallic Acid (GA/G) were added via the water phase. To obtain the different EDTA levels, EDTA-stock solution of $0.4 \%$ w/w in $\mathrm{H} 2 \mathrm{O}$ was replacing part of the water phase: respectively $0.25 \mathrm{~g}, 0.50 \mathrm{~g}$ and $1.88 \mathrm{~g}$ to obtain 10,20 and $75 \mathrm{ppm}$ EDTA. For the different GA concentrations, a GA-stock solution of $1 \% \mathrm{w} / \mathrm{w}$ in $\mathrm{H} 2 \mathrm{O}$ was added: respectively $3.0 \mathrm{~g}$ and $6.0 \mathrm{~g}$ to obtain 75 and $150 \mathrm{ppm}$ GA. The samples $75 \mathrm{E}-0 \mathrm{G}$ and $0 \mathrm{E}-0 \mathrm{G}$ and the centre sample $10 \mathrm{E}-75 \mathrm{G}$ were prepared in duplicate. For the accelerated shelf-life tests (ASLTs), two aliquots of $1 \mathrm{~g}$ mayonnaise per formulation were stored in $20 \mathrm{~mL}$ screwcap vials in the dark at $50{ }^{\circ} \mathrm{C}$ for shelf-life testing. Upon collection, the aliquots were then stored at $-20{ }^{\circ} \mathrm{C}$ until further analysis.

\subsubsection{NMR measurements}

For the quantification of hydroperoxides and aldehydes in these aliquots, the oil phase was first obtained by freeze-thawing the mayonnaise to break the emulsion followed by centrifugation (VWR, MicroStar 17R, the Netherlands) for 5 minutes at 17,000 $\mathrm{x}$ g to obtain a good separation of the oil and water layers. $150 \mu \mathrm{L}$ of the oil phase was dissolved in 450 $\mu \mathrm{L}$ 5:1 $\mathrm{CDCl}_{3}$ :DMSO-d6 solvent. The mixture $(600 \mu \mathrm{L})$ was transferred to 5-mm NMR 
tubes. Single pulse and band selective ${ }^{1} \mathrm{H}$ NMR spectra were then recorded on a $600 \mathrm{MHz}$ (14.1 T) Bruker Avance III NMR spectrometer (Bruker BioSpin, Switzerland). Details on the assignment of signals and quantification can be found in Merkx et al. (2018).

\subsubsection{Model selection and prediction data}

The data set that was used for the model selection was pooled from a collection of trials performed in-house, with 238 formulations in total. Out of this data, two groups were defined. A schematic overview of the formulations used in this study can be found in the Supporting Materials (Table S5.1). One overarching group of data sets (Set D in Table S5.1) contained all data that had $\mathrm{LOOH}$-curves at $50{ }^{\circ} \mathrm{C}$, with at least five time points measured in duplicate, that were used for the model selection steps. Of this overarching group, a subgroup consisting of 23 samples from three different trials (Set A, B and C in Table S5.1) were stored for more than 30 days and had complementary aldehyde data to be used for the prediction studies. First, set D was used for selecting the sigmoidal model that provides the best fit to the $\mathrm{LOOH}$ curves under ASLT (2.4.1 and 2.4.2). Next, data from sets A, B and C was used to build a model for predicting the aldehyde onset (2.4.3).

\subsubsection{Sigmoid models}

For the description of the LOOH generation, two sigmoidal functions were explored: the Gompertz (Gompertz, 1825) and Foubert (Foubert, Vanrolleghem, Vanhoutte, \& Dewettinck, 2002) functions, described in Eq. 5.1 and Eq. 5.2, respectively:

$$
\begin{gathered}
\mathrm{C}_{\mathrm{Ox}}=\mathrm{A} \cdot \mathrm{e}^{-\mathrm{e}^{\mathrm{K}(\mathrm{t}-\mathrm{t} 0)}} \\
\mathrm{C}_{\mathrm{Ox}}=-\mathrm{A} \cdot\left[1+\left(\left(\frac{\mathrm{A}-\mathrm{f} 0}{\mathrm{~A}}\right)^{1-\mathrm{n}}-1\right) \cdot \mathrm{e}^{-(1-\mathrm{n}) \cdot \mathrm{K} \cdot \mathrm{t}}\right]^{\frac{1}{1-\mathrm{n}}}-\mathrm{A}
\end{gathered}
$$

Here, $\mathrm{C}_{\mathrm{Ox}}$ is the concentration of oxidation product $(\mathrm{mmol} / \mathrm{kg}$ ) and $\mathrm{t}$ the storage time (day). Parameter A indicates the upper asymptote $(\mathrm{mmol} / \mathrm{kg})$ and parameter $\mathrm{K}$ the growth rate (day${ }^{1}$ ). For Eq. 5.1 (Gompertz), the parameter t0 is the inflection time in days of the curve (day). In Eq. 5.2 (Foubert), this inflection time is rewritten as f0, which can be interpreted as the initially present oxidation products $(\mathrm{mmol} / \mathrm{kg}$ ). Parameter $\mathrm{n}$ is a constant related to the asymmetry of the curve. The regression was done using the Excel Solver add-in using GRG Non Linear fitting with the boundaries as described in Table S5.2. 


\subsubsection{Model selection criteria}

To find the best model for describing hydroperoxide generation, three information criteria were evaluated. These three criteria were the Aikake Information Criterion (AIC), Bayesian Information Criterion (BIC) and Law of Iterated Logarithm Criterion (LILC) (Hannan \& Quinn, 1979). For every curve, the AIC, BIC and LILC values were collected. Here, the model with the lowest criterion-value was considered the best model. An F-test was performed to evaluate in how many cases the full Foubert model (F4, including four variable parameters) was significantly better than the reduced Foubert model (F3, three variable parameters and one fixed parameter (n)).

\subsubsection{Prediction model}

In the prediction model of the aldehyde onset time, the regressor $(\mathrm{t})$ was set at the time the $\mathrm{CC}_{\mathrm{LOOH}}$ reached a concentration of $38 \mathrm{mmol} / \mathrm{kg}$ (Eq. 5.3, rewritten form of Eq. 5.2). The predictor was the onset time of the aldehydes. The objective cross-point determination was adapted and slightly modified from Pinchuk and Lichtenberg (2014). A schematic overview of this procedure is shown in the supporting information (Figure S5.3). In brief, the aldehyde curvature was first described using regression with the reduced Foubert function using $\mathrm{n}=$ 1.1 (best model to describe aldehyde generation from the model selection; data not shown). From this, its derivative function (Eq. 5.4) was used to estimate the maximum slope (Sx) using the Excel Solver add-in. The intercept of this slope with the baseline, set at 1.0 $\mathrm{mmol} / \mathrm{kg}$, was interpreted as the onset time of the aldehydes. The prediction was performed using linear regression without transformation in the form of $y=a x$.

$$
\begin{aligned}
& \mathrm{t}=\ln \left(\frac{\left(\frac{-\mathrm{C}_{\mathrm{LOOH}}+\mathrm{A}}{\mathrm{A}}\right)^{\frac{1-\mathrm{n}}{1}}-1}{\left(\frac{\mathrm{A}-\mathrm{f0}}{\mathrm{A}}\right)^{1-\mathrm{n}}-1}\right) \cdot \frac{1}{(\mathrm{n}-1) \cdot \mathrm{K}} \\
& \mathrm{S}_{\mathrm{x}}=\mathrm{A}\left(\left(\frac{\mathrm{A}-\mathrm{f} 0}{\mathrm{~A}}\right)^{1-\mathrm{n}}-1\right) \cdot \mathrm{Ke}^{-(1-\mathrm{n}) \mathrm{Kx}}\left(\left(\left(\frac{\mathrm{A}-\mathrm{f0}}{\mathrm{A}}\right)^{1-\mathrm{n}}-1\right) \mathrm{e}^{-(1-\mathrm{n}) \mathrm{Kx}}+1\right)^{\frac{1}{1-\mathrm{n}}-1}
\end{aligned}
$$

Here, $t$ is the timepoint at which the hydroperoxide concentration $C_{\text {LOOH }}$ is reached (day). $S_{x}$ is the slope (first derivative) of Eq 5.2 ( $\mathrm{mmol} / \mathrm{kg} / \mathrm{day}$ ). Parameter A is the upper asymptote $(\mathrm{mmol} / \mathrm{kg}), \mathrm{K}$ the curvature constant $\left(\mathrm{day}-{ }^{1}\right)$, f0 the initially present oxidation products $(\mathrm{mmol} / \mathrm{kg}$ ) related to induction time, $\mathrm{n}$ the asymmetry constant of the Foubert function. 


\subsubsection{Model validation and chemical parameter interpretation}

A nonlinear least-squares curve-fitting procedure (lsqcurvefit) in Matlab R2020a (The MathWorks, Inc.) was used for estimating the model parameters A, K and f0 in the reduced Foubert function (Eq. 5.2). The standard error for each model parameter was determined via a bootstrap regression procedure with residual resampling (Efron \& Tibshirani, 1993) and included 1000 repetitions. As the statistical distribution of the model parameters was unknown, the (non-parametric) bootstrap was considered as the most appropriate approach in our error estimate. A two-way ANCOVA (ANalysis of COVAriance) was performed to establish whether the contribution of different AOs on parameters $\mathrm{K}$ and f0 were statistically significant (at $\alpha=0.05$ ). Prior to the ANCOVA, both K and f0 were log-transformed to adjust for non-normality in the data. The regression coefficients of the ANCOVA model were used to determine the degree of association between the AOs and the dependent variables $\mathrm{K}$ and f0 (Eq. 5.5 and 5.6). Since the AOs were in the same concentration regime, the absolute size of these model coefficients could directly be interpreted as (relative) effect sizes. These analyses were done with $\log$ transformation of f0 and $\mathrm{K}$ in $\mathrm{R}$ (R Core Team, 2020) and the $\operatorname{lm}$ function in the stats (v3.6.2) package.

$$
\begin{array}{lr}
\mathrm{K}=\mu+\alpha \mathrm{AO}_{\mathrm{i}}+\beta \mathrm{AO}_{\mathrm{j}}+\gamma \mathrm{AO}_{\mathrm{i}} \mathrm{AO}_{\mathrm{j}}+\varepsilon & \text { Eq. } 5.5 \\
\mathrm{f} 0=\mu+\alpha \mathrm{AO}_{\mathrm{i}}+\beta \mathrm{AO}_{\mathrm{j}}+\gamma \mathrm{AO}_{\mathrm{i}} \mathrm{AO}_{\mathrm{j}}+\varepsilon & \text { Eq. } 5.6
\end{array}
$$

Here, $\mathrm{K}$ and $\mathrm{f0}$ are the dependent variables (Eq. 5.2), $\mu$ the mean, $\alpha, \beta$ and $\gamma$ the regression coefficients $\left(\mathrm{ppb}^{-1}\right)$ of the corresponding term and $\varepsilon$ the residual error. AO stands for the antioxidant (ppb), with the indexes $\mathrm{i}$ and $\mathrm{j}$ for the type of AO.

\subsection{Results and Discussion}

\subsubsection{Justification of sigmoidal models to describe hydroperoxide formation}

For the analysis of lipid oxidation products in food emulsions we used a binary solvent mixture, Typical curves of hydroperoxide generation during an accelerated shelf-life test (ASLT) are shown in Figure 5.1A. The three curves represent mayonnaises prepared with different antioxidant (AO) levels and follow a sigmoidal curvature up to a concentration of around $38 \mathrm{mmol} / \mathrm{kg}$. From here onwards, the LOOH generation enters a second acceleration phase. The initiation of this secondary acceleration is believed to be related to the generation of aldehydes. This secondary acceleration can be explained by the recently introduced theoretical framework (Budilarto \& Kamal-Eldin, 2015; Laguerre et al., 2020), which suggests that at certain concentrations surface active hydroperoxides from inverse micelles 
that propagate the oxidation reaction in a quicker rate. This aligns with our observations and we therefore refer this concentration when this secondary acceleration is observed (38 $\mathrm{mmol} / \mathrm{kg}$ ) as the Critical Concentration of LOOHs $\left(\mathrm{CC}_{\mathrm{LOOH}}\right)$.

A

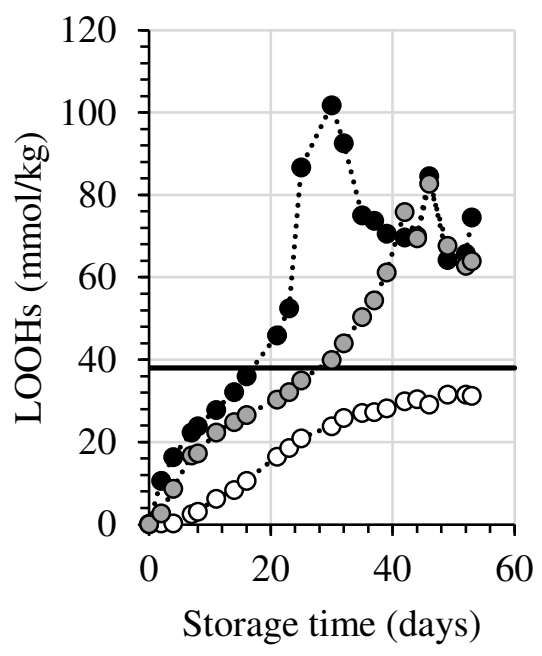

B

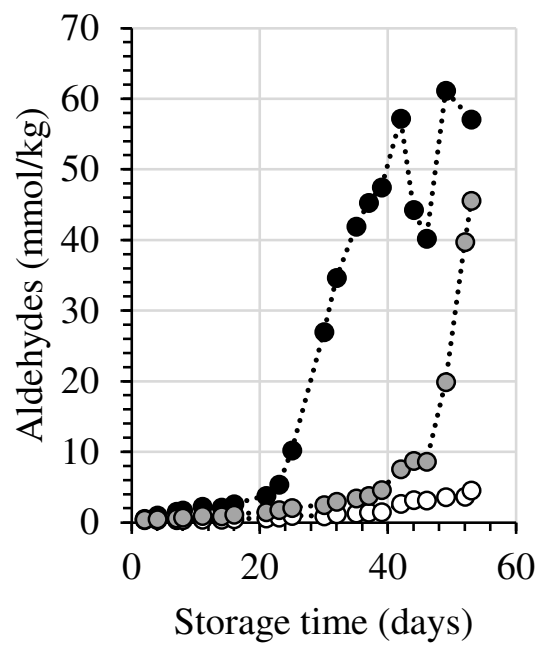

Figure 5.1. Typical profile of hydroperoxides (LOOHs) (A) and aldehyde (B) generation over time under accelerated shelf-life conditions $\left(50^{\circ} \mathrm{C}\right)$ measured by ${ }^{1} \mathrm{H}$ NMR. Three different formulations are displayed: no EDTA (black), EDTA-alternative (grey), EDTA (white). In A, a line is drawn at $38 \mathrm{mmol} / \mathrm{kg}$, the estimated critical concentration of $\mathrm{LOOHs}\left(\mathrm{CC}_{\mathrm{LOOH}}\right)$, after which the secondary acceleration is engaged. Lines are solely drawn with the purpose of guiding the eye (no fit).

To quantitatively describe these sigmoidal initial phases of $\mathrm{LOOH}$ generation (up to the $\mathrm{CC}_{\mathrm{LOOH}}$ ), two model functions were explored: the Gompertz (Gompertz, 1825) and the recently developed Foubert (Foubert et al., 2002) function. The Foubert function is an adaptation of the Gompertz function that includes a parameter for asymmetry. The inclusion of an asymmetry parameter allows for a better description of processes that have competing reactions. Foubert et al. developed this to describe crystal growth, where besides growth and sigmoid progression, they also needed to consider the reverse reaction. In lipid oxidation, the degradation of LOOHs can similarly reduce the symmetry of the sigmoidal curve. Furthermore, other parameters that describe the shape of the sigmoidal curve, such as induction time $(\mathrm{t} 0 / \mathrm{f} 0)$ and growth rate $(\mathrm{K})$, can hold information regarding the oxidative mechanisms at play and will be investigated. For example, a radical scavenger antioxidant will hypothetically have a bigger impact on the induction time than a metal chelating 
antioxidant. The latter will most likely primarily limit the growth rate since it reduces the amount of pro-oxidative transition metal in the system.

\subsubsection{Selection and training of sigmoidal models}

For model selection, we excluded LOOH-concentrations above $40 \mathrm{mmol} / \mathrm{kg}$, which was slightly above the estimated $\mathrm{CC}_{\mathrm{LOOH}}$ of $38 \mathrm{mmol} / \mathrm{kg}$, since these clearly would not fit the sigmoidal function. Both the Gompertz (G, Eq. 5.1) and normal Foubert (F4, Eq. 5.2) functions were fitted to hydroperoxide generation curves acquired for a wide range of mayonnaise formulations stored at $50{ }^{\circ} \mathrm{C}$ ( 238 curves). The boundaries of the parameters are described in Table S5.2, with most notably the fitting boundaries for the asymptote (A) for both functions. Here, the data was fitted only with an A variation from 50 to $70 \mathrm{mmol} / \mathrm{kg}$ to ensure all curve-fittings did not reach their limit before the estimated $\mathrm{CC}_{\mathrm{LOOH}}$ of $38 \mathrm{mmol} / \mathrm{kg}$. The G and F4 functions were compared by determining the AIC, BIC and LILC criteria for each curve. These criteria increasingly compensate for the number of samples in the series (LILC > BIC > AIC, section 2.6.). According to all three criteria, the Foubert function fitted the data better than the Gompertz functions (Table 5.1).

As mentioned before, the Foubert function included a parameter $\mathrm{n}$ that accounts for the asymmetry of the system. We hypothesised that this asymmetry was similar for all tested systems as they underwent the same ASLT. To estimate a good $n$ value, the average of $n$ in a variety of sample series were taken and tested throughout the whole data set. It was found that for $n=16.7$ (Table S5.4) the model matches the ASLT conditions, i.e. shelf-life tests performed at $50{ }^{\circ} \mathrm{C}$. By setting the $\mathrm{n}$ to a fixed number, the number of independent variables in the Foubert function were reduced from four to three. This does again impact the assessment on the quality of the fit. So, the criterion comparisons were repeated including the reduced Foubert function (F3) with $n=16.7$. According to the AIC and BIC, F3 was the best fit in over half of the curves, with F4 a close second (Table 5.2). For the LILC criterion, F4 and F3 did not differ. According to all criteria, the Gompertz function was the poorest fitting function. To estimate whether the difference between the two Foubert functions was significant, an F-test was performed. Respectively, for $\mathrm{p}=0.05$ and $\mathrm{p}=0.01$, in $68 \%$ and $77 \%$ of the cases, the F4-function was not significantly better in fitting the data than the F3function. We were therefore confident to use the reduced Foubert (F3) in the following prediction and validation studies (Eq. 5.2, with $\mathrm{n}=16.7$ ). 
Table 5.1. Comparison of information criteria AIC, BIC and LILC for the models G, F3 and F4. The displayed numbers represent the number of curves (within the 238 curves tested) that presented the lowest criterion-value for each model.

\begin{tabular}{ccccc}
\hline & Gompertz $(G)$ & Normal Foubert (F4) & Reduced Foubert (F3) & Order model qualities \\
\hline AIC & 18 & 98 & 122 & F3 $>$ F4 $>>$ G \\
BIC & 19 & 92 & 127 & F3 $>$ F4 $>$ G \\
LILC & 17 & 111 & 110 & F3 $\approx$ F4 $>>$ G \\
\hline
\end{tabular}

\subsubsection{Aldehyde onset prediction}

Now that a function has been selected to quantitatively describe the LOOH-generation under accelerated conditions, we pursued the prediction of the time of the aldehyde onset. This prediction model was set up using data sets that comprised both the LOOH curves and aldehydes curves that showed a clear onset. These curves could be primarily grouped in three data sets (A, B, C) according to oil levels (65, 75 and 78\%, more detail in Table S5.1). To establish a robust prediction model that allows variation in base formulations, the three different groups were treated as one data set. The aldehyde onset for each curve was objectively determined by using the cross-point determination method (see 2.4.3). In our three different formulation groups, the $\mathrm{CC}_{\mathrm{LOOH}}$, the hydroperoxide concentration at which the second acceleration started, differed more than $10 \mathrm{mmol} / \mathrm{kg}$, from 38 to $50 \mathrm{mmol} / \mathrm{kg}$. Figure $5.2 \mathrm{~A}$ shows the time difference $\left(\Delta \mathrm{t}_{\mathrm{CC}-\mathrm{AO}}\right)$ between the moment the $\mathrm{CC}_{\mathrm{LOOH}}$ was reached $\left(\mathrm{t}_{\mathrm{CC}}\right)$, and the aldehyde onset $\left(\mathrm{t}_{\mathrm{AO}}\right)$. The $\Delta \mathrm{t}_{\mathrm{CC}-\mathrm{AO}}$ values differed between, and within the formulation groups. Hence, there was no constant delay between reaching $\mathrm{CC}_{\mathrm{LOOH}}$ and the onset of aldehyde generation.

Therefore, we opted for a more robust and empirical approach. Instead of looking at the time difference, we directly looked at the time at which a $\mathrm{CC}_{\mathrm{LOOH}}$ was reached. And instead of pinpointing the exact $\mathrm{CC}_{\mathrm{LOOH}}$, the value on the lower end $(38 \mathrm{mmol} / \mathrm{kg})$ of the observed $\mathrm{CC}_{\mathrm{LOOH}}\left(38-50 \mathrm{mmol} / \mathrm{kg}\right.$ ) was used and the time at which this $\mathrm{CC}_{\mathrm{LOOH}}$ of $38 \mathrm{mmol} / \mathrm{kg}$ was reached was correlated with the aldehyde onset. Linear regression showed that the aldehyde onset can be predicted from the time at which $\mathrm{CC}_{\mathrm{LOOH}}$ of $38 \mathrm{mmol} / \mathrm{kg}$ is reached (Figures $2 \mathrm{~B}$, S5). This approach implies a longer delay between the aldehyde onset and $\mathrm{CC}_{\mathrm{LOOH}}$ time if the formulation is more stable, i.e. due to the presence of more antioxidants or less prooxidants. 

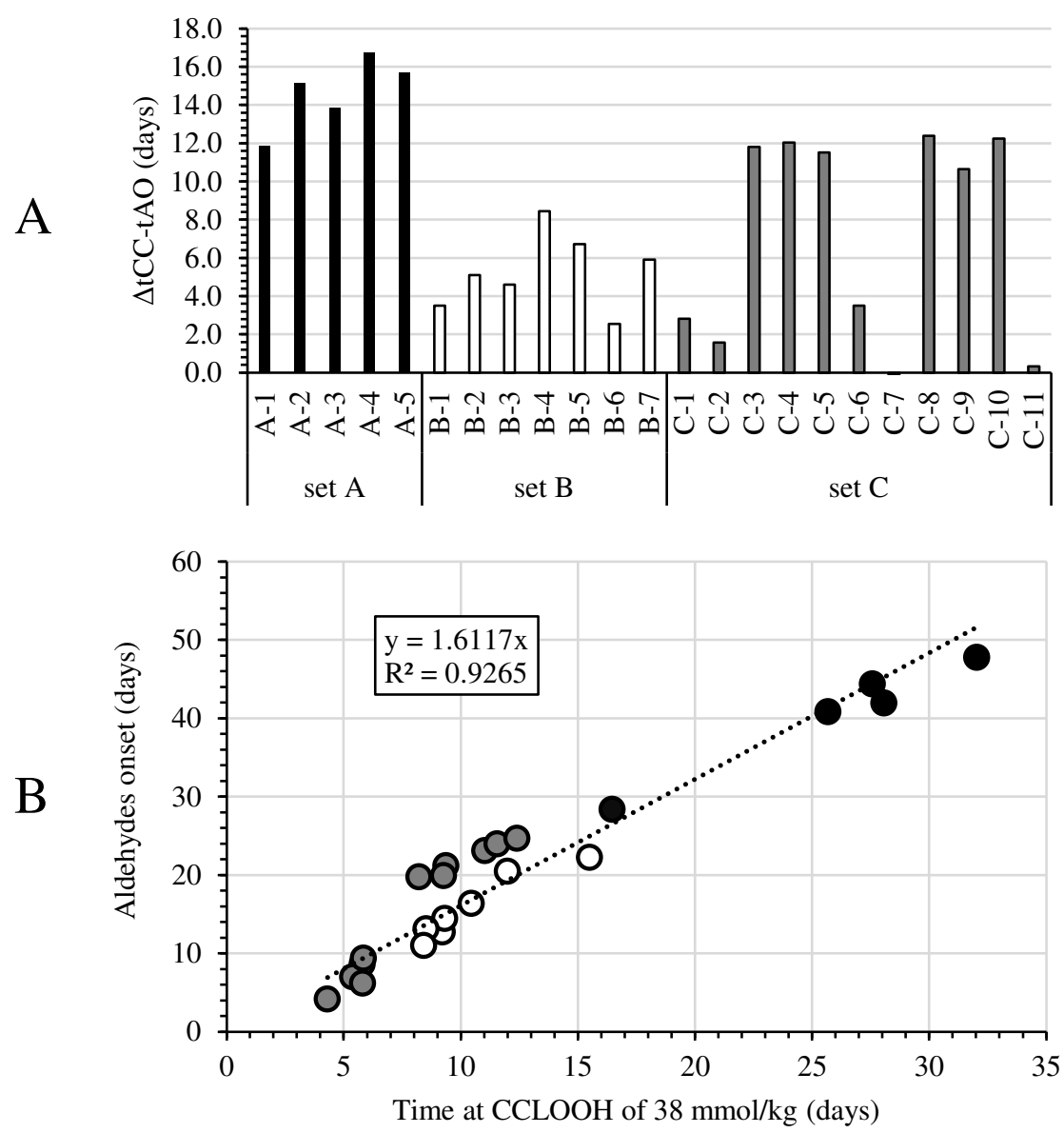

Figure 5.2. Dependencies between the time after which the $\mathrm{CC}_{\mathrm{LOOH}}$ was reached and the onset of the corresponding aldehyde curve. Different colours represent the different oil contents in the mayonnaises (set A (65\% rapeseed (RP)) black; set B (75\% RP) white; set C (78\% RP) gray). A: Time differences between the moment $\mathrm{CC}_{\mathrm{LOOH}}$ was reached and the aldehyde onset $\left(\Delta \mathrm{t}_{\mathrm{CC}-\mathrm{AO}}\right)$, where every bar represents a formulation within the mayonnaise set. B: Linear regression model with the time at $\mathrm{CC}_{\mathrm{LOOH}}$ as regressor and aldehyde onset as predictor where every circle represents a formulation within the mayonnaise sample set. 


\subsubsection{Model validation}

Lastly, we validated the Foubert (F3) model and its predictive performance for the onset of aldehydes. A data set with mayonnaises containing both primary and secondary antioxidants (AOs) was designed and the oxidation under ASLT conditions was tracked until the aldehyde onset was reached for most of the formulations. We included three duplications to assess the reproducibility of the method. The AOs that were chosen were GA as a primary AO-system, and EDTA as a secondary AO-system. In this setup, both AOs were dosed in three different levels, in every combination. Since GA and EDTA have different AO-efficiencies, they were dosed in different increments (GA per $75 \mathrm{ppm}$, EDTA per $10 \mathrm{ppm}$ ) to obtain a more comparable effect on overall oxidation. The data set covered a wide range of $\mathrm{LOOH}$ curvatures (Figures 3, S6). This confirmed that the AOs were dosed in a working range allowing to probe adequately the contribution of the different AO systems. For all formulations, the curves reached $40 \mathrm{mmol} / \mathrm{kg}$ of $\mathrm{LOOH}$, so both sigmoidal progression and the secondary acceleration stage were covered. Variation between duplicate curves was very low, both on formulation level and sample level (both $<5 \%$ ). For the aldehyde curves (Figure 5.3, S5.4), the differentiation between the samples was also present. All, except the $75 \mathrm{ppm}$ EDTA-formulations, reached a clear onset point and could therefore be used for the validation of the aldehyde-onset-prediction model (Figure 5.4, open bars).

\section{Sum of hydroperoxides}

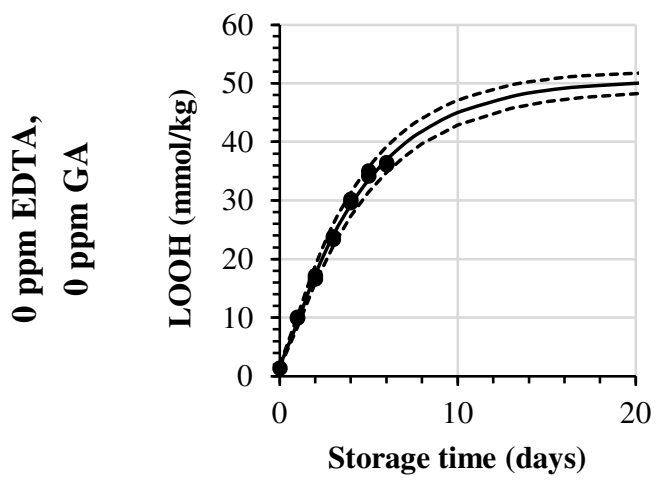

Sum of aldehydes

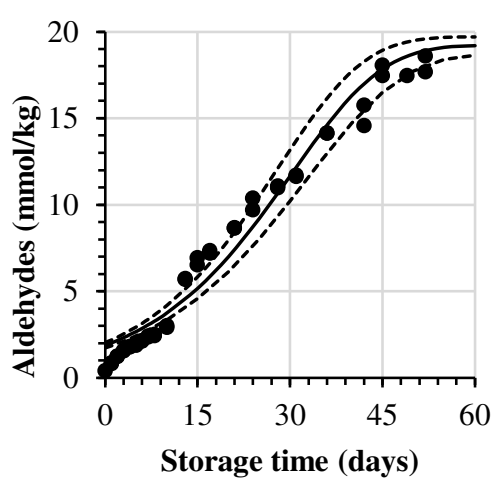



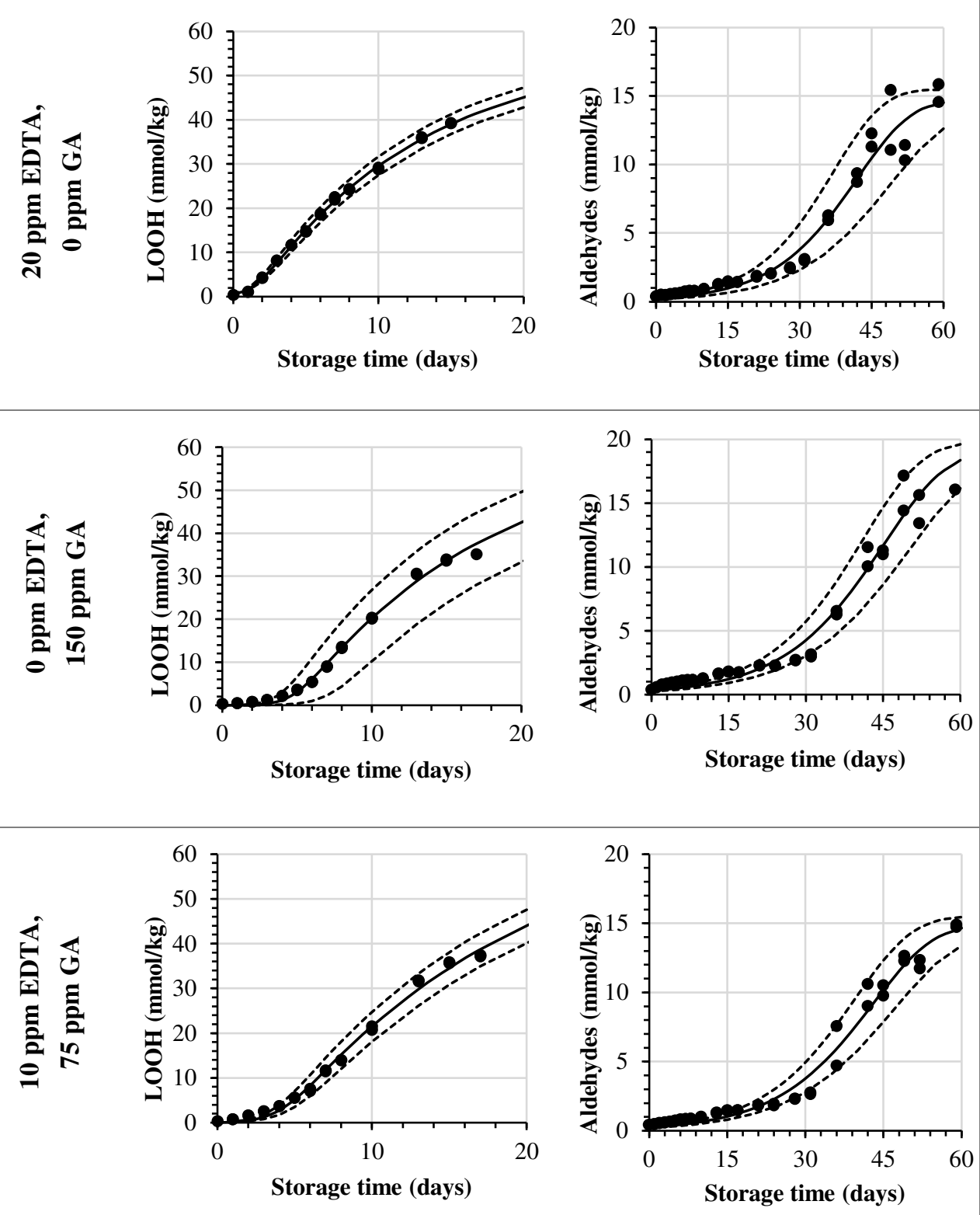

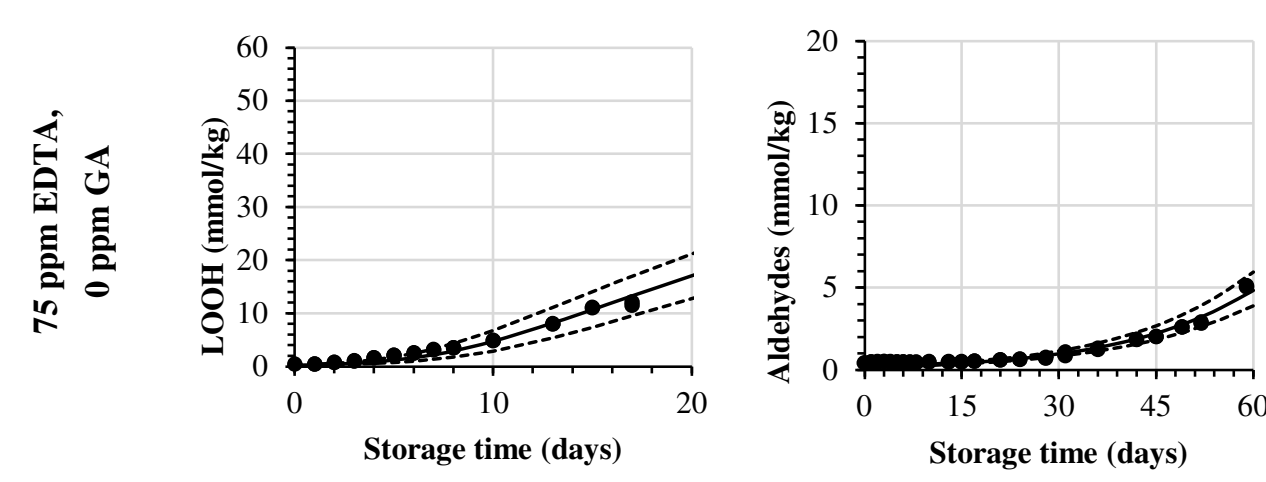

Figure 5.3. Selection of reduced Foubert (F3) regression results. The dots are the measured data points by ${ }^{1} \mathrm{H}$ NMR, the solid line corresponds to the F3 function, and the dashed lines represent the $95 \%$ confidence interval (CI). The left graphs represent the LOOH-data up to $40 \mathrm{mmol} / \mathrm{kg}$, fitted with the F3 function with $\mathrm{n}=16.7$. The right graphs represent the aldehyde-data, fitted with the F3 function with $\mathrm{n}=1.1$ to be used for the cross-point determination.

In the previous section, we proposed a simple linear regression $(\mathrm{y}=1.6 \mathrm{x})$ to predict the onset of aldehydes based on the time at which $\mathrm{CC}_{\mathrm{LOOH}}$ was reached using the $\mathrm{F} 3$ model. Two different approaches of describing the LOOH-data were explored to predict the aldehyde onset. First, as with the original data, all $\mathrm{LOOH}$ concentrations below $40 \mathrm{mmol} / \mathrm{kg}$ were used to fit the model (Eq. 5.2). From this model, the storage time after which the function approached the proposed $\mathrm{CC}_{\mathrm{LOOH}}(38 \mathrm{mmol} / \mathrm{kg})$ was used to predict the aldehyde onset (Figure 5.4, solid bars). In all eleven formulations, no significant difference $(\mathrm{p}<0.05)$ was observed between the predicted and measured aldehyde onsets. In the second approach, only the data from the first week (7 days with daily sampling) were used to build the LOOH-model (Figure 5.4, dashed bars). For nine out of the eleven formulations, the predicted aldehyde onset was not significantly different from the measured aldehyde onset $(\mathrm{p}<0.05)$. However, in the majority of the models, the standard error was visibly bigger than when using the full data. In two cases, the predicted onsets were significantly different than the measured ones ( $\mathrm{p}<0.05$ ). Both these formulations consisted of $150 \mathrm{ppm}$ GA, which hampers $\mathrm{LOOH}$ generation in the early stages of the process and did not show a clear sigmoidal curve in the first 7 days. This lack of early data points limited the accuracy of the F3 function. For both approaches, the duplicate formulations were not significantly different from one another $(\mathrm{p}$ $<0.01$ ). In summary, we have shown that the time at which a $\mathrm{CC}_{\mathrm{LOOH}}$ of $38 \mathrm{mmol} / \mathrm{kg}$ is reached can be used to predict the aldehyde onset. This prediction model allows us to accurately predict the onset of aldehydes by using hydroperoxide data measured in the first 7 days. The accuracy of the prediction is limited in systems with a high load of AO. In those cases, it would be advised to measure beyond the 7 days mark. This means that early LOOH 
measurements can be used to predict compromised shelf-life instead of using analytical timeconsuming determination of the onset of aldehydes, such as hexanal measurements. The correlation between $\mathrm{CC}_{\mathrm{LOOH}}$ and aldehyde onsets is in line with our hypothesis that secondary oxidation is accelerated once micelles are formed. We note that correlation does not establish causality.

\subsubsection{Chemical interpretation of the model parameters $\mathrm{K}$ and $\mathrm{fO}$}

The same data set was used for the chemical interpretation of the two main describing parameters of the model, $\mathrm{K}$ and f0 (overview in Figure S5.5). The f0 (measure for induction delay) and $\mathrm{K}$ (growth rate), were respectively hypothesised to be linked to primary (radical scavenging) and secondary (metal chelating) antioxidant effects. Two-way ANCOVA (Eq. 5.5 and 5.6), was performed to identify the significant $\mathrm{AO}$ factors to f0 and $\mathrm{K}$ (Table 5.2). No significant $(\mathrm{p}<0.05)$ interaction-effect between the two AOs on both response parameters was observed, which meant that GA and EDTA did not significantly influence each other in their AO-mechanisms. We observed that GA and EDTA had significant contributions on both $\mathrm{f} 0$ and $\mathrm{K}$ ( $\mathrm{p}$-values in Table 5.2). The absolute values of the ANCOVA regression model $\left([\mathrm{ppb}]^{-1}\right)$ represented the relative effects that GA and EDTA had on these two response parameters.

For f0, the effect of EDTA and GA per ppb was not significantly different from each other $(p<0.05)$. This meant that on weight basis, the effects of EDTA and GA were similar and that $\mathrm{fO}$ is not only sensitive for radical scavenging effects (GA) but also for the chelation effect (EDTA) in the early stages of lipid oxidation. This latter observation is in line with recent work that describes that in the early state of oxidation, $\mathrm{LOOH}$ can be generated via LH radicals as well as iron redox cycling (Laguerre et al., 2020; Merkx et al., 2021). 


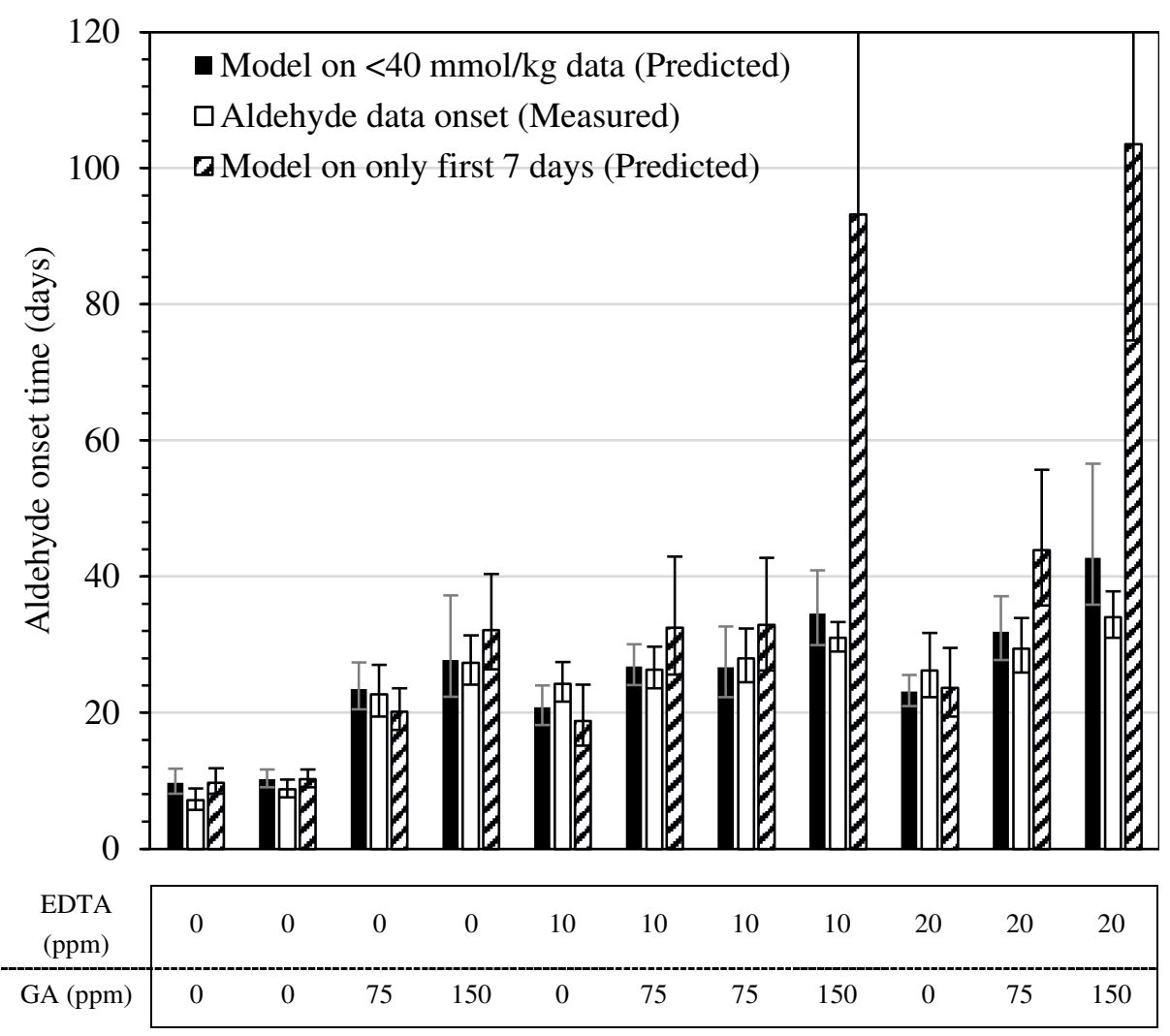

Figure 5.4. Overview of the predicted and measured aldehyde onset times of the mayonnaises from the model validation trial stored at $50{ }^{\circ} \mathrm{C}$ under accelerated shelf-life test (ASLT) conditions. The predicted aldehyde onset based on the reduced Foubert (F3) function and the prediction model (Figure 5.2B) was determined using all data $<40 \mathrm{mmol} / \mathrm{kg}$ (closed bars) and only the first seven days (dashed bars). Open bars are the onset values based on the cross-point determination obtained from the measured sum of aldehydes. All data was measured with ${ }^{1} \mathrm{H}$ NMR.

For K, a much stronger effect was observed for EDTA, supporting our hypothesis that the main effect of K can be explained by secondary AO-effects. Increasing the amount of EDTA lowers the effective pro-oxidant iron, thereby slowing the reaction. The small, but significant effect of GA on $\mathrm{K}$ is in line with this compound being a weak chelator for iron (Andjelković et al., 2006). Our observations suggest that our modelling approach can be used to indicate the primary mode of action (primary vs. secondary) of an AO that is added to the emulsion system. 
Table 5.2. Two-way analysis of covariance (ANCOVA) results, after log-transformation. The reported values in this table represent the regression coefficients of the ANCOVA model [ppb] $]^{-1}$, which can be interpreted as the effect sise of each $\mathrm{AO}$ on the response parameters f0 and $\mathrm{K}$.

\begin{tabular}{lll}
\hline & \multicolumn{2}{l}{ Regression coefficient of } \\
& $\mathrm{f} 0[\mathrm{ppb}]^{-1}$ & $\mathrm{~K}[\mathrm{ppb}]^{-1}$ \\
\hline EDTA & $-64.4 \pm 40.3 * *$ & $-50.0 \pm 20.3 * * *$ \\
Gallic Acid & $-33.2 \pm 5.4 * * *$ & $-7.2 \pm 2.7 * * *$ \\
\hline$* * \mathrm{p}<0.01, * * * \mathrm{p}<0.001$ & &
\end{tabular}

\subsection{Conclusion}

A sigmoidal Foubert-adapted model can be used to describe the initial phase of hydroperoxide generation, measured by NMR, under an accelerated shelf-life condition (50 ${ }^{\circ} \mathrm{C}$ ). The point at which this function reaches the proposed $\mathrm{CC}_{\mathrm{LOOH}}$ of $38 \mathrm{mmol} / \mathrm{kg}$ can be used as a predictor for the onset of aldehyde generation. This prediction can be applied in different mayonnaise formulations. In most cases, $\mathrm{LOOH}$ measurements in the first week of shelf-life are sufficient to accurately predict the aldehyde onset. They could therefore provide an early and quantitative assessment of compromised shelf-life stability. Furthermore, the parameters that describe the Foubert function, i.e., the induction delay (f0) and the growth rate $(\mathrm{K})$, can be used to discriminate primary and secondary anti-oxidative mechanisms. 


\subsection{Literature}

Andjelković, M., Van Camp, J., De Meulenaer, B., Depaemelaere, G., Socaciu, C., Verloo, M., \& Verhe, R. (2006). Iron-chelation properties of phenolic acids bearing catechol and galloyl groups. Food Chemistry, 98(1), 23-31.

Berton, C., Ropers, M.-H., Bertrand, D., Viau, M., \& Genot, C. (2012). Oxidative stability of oil-in-water emulsions stabilised with protein or surfactant emulsifiers in various oxidation conditions. Food Chemistry, 131(4), 1360-1369.

Brimberg, U. I., \& Kamal-Eldin, A. (2003). On the kinetics of the autoxidation of fats: influence of pro-oxidants, antioxidants and synergists. European Journal of Lipid Science and Technology, 105(2), 83-91.

Budilarto, E. S., \& Kamal-Eldin, A. (2015). The supramolecular chemistry of lipid oxidation and antioxidation in bulk oils. Eur J Lipid Sci Technol, 117(8), 1095-1137.

Calligaris, S., Manzocco, L., Anese, M., \& Nicoli, M. C. (2016). Shelf-life Assessment of Food Undergoing Oxidation-A Review. Critical Reviews in Food Science and Nutrition, 56(11), 1903-1912.

Chaiyasit, W., Elias, R. J., McClements, D. J., \& Decker, E. A. (2007). Role of physical structures in bulk oils on lipid oxidation. Crit Rev Food Sci Nutr, 47(3), 299-317.

Chaiyasit, W., McClements, D. J., Weiss, J., \& Decker, E. A. (2008). Impact of SurfaceActive Compounds on Physicochemical and Oxidative Properties of Edible Oil. Journal of Agricultural and Food Chemistry, 56(2), 550-556.

Chen, B., Han, A., Laguerre, M., McClements, D. J., \& Decker, E. A. (2011). Role of reverse micelles on lipid oxidation in bulk oils: impact of phospholipids on antioxidant activity of $\alpha$-tocopherol and Trolox. Food \& Function, 2(6), 302-309.

Chen, B., Panya, A., McClements, D. J., \& Decker, E. A. (2012). New insights into the role of iron in the promotion of lipid oxidation in bulk oils containing reverse micelles. J Agric Food Chem, 60(13), 3524-3532.

Efron, B., \& Tibshirani, R. J. (1993). An Introduction to the Bootstrap: Chapman and Hall/CRC.

Foubert, I., Dewettinck, K., \& Vanrolleghem, P. A. (2003). Modelling of the crystallization kinetics of fats. Trends in Food Science \& Technology, 14(3), 79-92.

Foubert, I., Vanrolleghem, P. A., Vanhoutte, B., \& Dewettinck, K. (2002). Dynamic mathematical model of the crystallization kinetics of fats. Food Research International, 35(10), 945-956.

Frankel, E. N., Hu, M.-L., \& Tappel, A. L. (1989). Rapid headspace gas chromatography of hexanal as a measure of lipid peroxidation in biological samples. Lipids, 24(11), 976.

Gómez-Alonso, S., Mancebo-Campos, V., Desamparados Salvador, M., \& Fregapane, G. (2004). Oxidation kinetics in olive oil triacylglycerols under accelerated shelf-life testing $\left(25-75^{\circ} \mathrm{C}\right)$. European Journal of Lipid Science and Technology, 106(6), 369-375.

Gompertz, B. (1825). On the Nature of the Function Expressive of the Law of Human Mortality, and on a New Mode of Determining the Value of Life Contingencies. Philos Trans. R. Soc. London, 115(513), 252-253.

Gutteridge, J. M. C., Richmond, R., \& Halliwell, B. (1979). Inhibition of the iron-catalysed formation of hydroxyl radicals from superoxide and of lipid peroxidation by desferrioxamine. Biochemical Journal, 184(2), 469-472. 
Hannan, E. J., \& Quinn, B. G. (1979). The Determination of the Order of an Autoregression. Journal of the Royal Statistical Society: Series B (Methodological), 41(2), 190-195.

Hollebrands, B., Varvaki, E., Kaal, S., \& Janssen, H.-G. (2018). Selective labeling for the identification and semi-quantification of lipid aldehydes in food products. Analytical and Bioanalytical Chemistry, 410(22), 5421-5429.

ISO 3960:2017. (2017). In Animal and vegetable fats and oils -- Determination of peroxide value -- Iodometric (visual) endpoint determination (pp. 10). Geneva: International Organization for Standardization.

Jacobsen, C., Sørensen, A. D. M., \& Nielsen, N. S. (2013). 4 - Stabilization of omega-3 oils and enriched foods using antioxidants. In C. Jacobsen, N. S. Nielsen, A. F. Horn, \& A.-D. M. Sørensen (Eds.), Food Enrichment with Omega-3 Fatty Acids (pp. 130149). Cambridge, United Kingdom: Woodhead Publishing.

Johnson, D. R., \& Decker, E. A. (2015). The Role of Oxygen in Lipid Oxidation Reactions: A Review. Annual Review of Food Science and Technology, 6(1), 171-190.

Laguerre, M., Tenon, M., Bily, A., \& Birtic, S. (2020). Toward a Spatiotemporal Model of Oxidation in Lipid Dispersions: A Hypothesis-Driven Review. Eur. J. Lipid Sci. Technol., 122, 1900209.

Manzocco, L., Calligaris, S., Anese, M., \& Nicoli, M. C. (2016). Chapter 2 - Determination and Prediction of Shelf Life of Oils/Fats and Oil/Fat-Based Foods. In M. Hu \& C. Jacobsen (Eds.), Oxidative Stability and Shelf Life of Foods Containing Oils and Fats (pp. 133-156): AOCS Press.

Merkx, D. W. H., Hong, G. T. S., Ermacora, A., \& van Duynhoven, J. P. M. (2018). Rapid Quantitative Profiling of Lipid Oxidation Products in a Food Emulsion by 1H NMR. Analytical Chemistry, 90(7), 4863-4870.

Merkx, D. W. H., Plankensteiner, L., Yu, Y., Wierenga, P. A., Hennebelle, M., \& Van Duynhoven, J. P. M. (2021). Evaluation of PBN spin-trapped radicals as early markers of lipid oxidation in mayonnaise. Food Chem, 334, 127578.

Pinchuk, I., \& Lichtenberg, D. (2002). The mechanism of action of antioxidants against lipoprotein peroxidation, evaluation based on kinetic experiments. Progress in Lipid Research, 41(4), 279-314.

Pinchuk, I., \& Lichtenberg, D. (2014). Analysis of the kinetics of lipid peroxidation in terms of characteristic time-points. Chemistry and Physics of Lipids, 178, 63-76.

Ragnarsson, J. O., \& Labuza, T. P. (1977). Accelerated shelf-life testing for oxidative rancidity in foods-A review. Food Chemistry, 2(4), 291-308.

Schröder, A., Sprakel, J., Boerkamp, W., Schroën, K., \& Berton-Carabin, C. C. (2019). Can we prevent lipid oxidation in emulsions by using fat-based Pickering particles? Food Research International, 120, 352-363.

Uluata, S., McClements, D. J., \& Decker, E. A. (2015). How the Multiple Antioxidant Properties of Ascorbic Acid Affect Lipid Oxidation in Oil-in-Water Emulsions. Journal of Agricultural and Food Chemistry, 63(6), 1819-1824.

Velasco, J., Andersen, M. L., \& Skibsted, L. H. (2005). Electron Spin Resonance Spin Trapping for Analysis of Lipid Oxidation in Oils: Inhibiting Effect of the Spin Trap alpha-Phenyl-N-tert-butylnitrone on Lipid Oxidation. Journal of Agricultural and Food Chemistry, 53, 1328-1336.

Zbikowska, A., Kozlowska, M., Poltorak, A., Kowalska, M., Rutkowska, J., \& Kupiec, M. (2018). Effect of addition of plant extracts on the durability and sensory properties of oat flake cookies. Journal of Thermal Analysis and Calorimetry, 134(2), 11011111. 


\subsection{Supporting information}

Table S5.1. Schematic overview of the different mayonnaise formulation sets used in this study.

\begin{tabular}{|c|c|c|c|c|}
\hline & \multicolumn{3}{|c|}{$\begin{array}{l}\text { Both } \mathrm{LOOH}>40 \mathrm{mmol} / \mathrm{kg} \text { data and aldehyde data } \\
\text { beyond onset for prediction model selection (5.3.3.) }\end{array}$} & \multirow{2}{*}{$\begin{array}{l}\text { LOOH data only to } \\
\text { used for model } \\
\text { selection }(5.3 .2 .)\end{array}$} \\
\hline \multirow{2}{*}{ Ingredient } & $\begin{array}{l}\text { set } \mathrm{A} \\
(5 \text { form. })\end{array}$ & $\begin{array}{l}\text { set B } \\
\text { (7 form.) }\end{array}$ & $\begin{array}{l}\text { set } \mathrm{C} \\
(11 \text { form.) }\end{array}$ & \\
\hline & \multicolumn{4}{|l|}{$\% \mathrm{w} / \mathrm{w}$} \\
\hline Rapeseed oil & 65.0 & 75.0 & 78.0 & $65-78$ \\
\hline Water & 22.1 & 12.0 & 14.8 & $12-22$ \\
\hline Sucrose & 1.3 & 1.3 & - & $0-1.3$ \\
\hline $\mathrm{NaCl}$ & 1.8 & 1.8 & 1.8 & 1.8 \\
\hline Vinegar spirit $12 \%$ & 2.7 & 2.6 & 0.7 & $0.7-2.6$ \\
\hline Egg blend (whole egg + egg yolk) & 6.7 & 6.7 & - & $0-6.7$ \\
\hline Egg yolk & 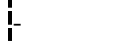 & - & 4.6 & $0-4.6$ \\
\hline $\mathrm{CaNa}_{2} \mathrm{EDTA}$ & $0-0.0075$ & $0-0.0075$ & $0-0.0075$ & $0-0.0075$ \\
\hline $\begin{array}{l}\text { Other chemical antioxidants (BHT, } \\
\text { BHT, chlorogenic acid) }\end{array}$ & 0 & 0 & 0 & $0-0.02$ \\
\hline Natural antioxidants (plant extracts) & $0-5$ & $0-5$ & 0 & $0-5$ \\
\hline
\end{tabular}


Table S5.2. Overview of the boundaries using for the fitting of both Gompertz (G) and Foubert (F) functions.

Lower limit Upper limit

\begin{tabular}{ccc}
\hline A (both G and F) & 50 & 70 \\
\hline $\mathrm{K}$ (both G and F) & 0 & 0.5 \\
\hline $\mathrm{T} 0(\mathrm{G})$ & 0 & 5 \\
\hline $\mathrm{f} 0(\mathrm{~F})$ & 0 & 2 \\
\hline $\mathrm{n}(\mathrm{F})$ & 0 & 75 \\
\hline
\end{tabular}

Table S5.3. BIC on the F4 model with different n-values. The displayed number represents the number of curves that have that model as the lowest criterion-value.

\begin{tabular}{l|llll}
\multicolumn{1}{c}{$\mathrm{n}=8$} & $\mathrm{n}=16.7$ & $\mathrm{n}=25.8$ & $\mathrm{n}=36$ \\
\hline BIC & 45 & 109 & 31 & 56
\end{tabular}

Figure S5.1. Schematic overview on the cross-point determination. On the left, the squares represent the concentration of aldehydes measured over time. The dashed line the reduced Foubert $(n=1.1)$ fit. The green line is the baseline, set at Cald $=1$. The orange line is the result of the maximum slope determination using Eq. 5.4, as shown in the right graph. The onset was determined by crossing the orange and green line.
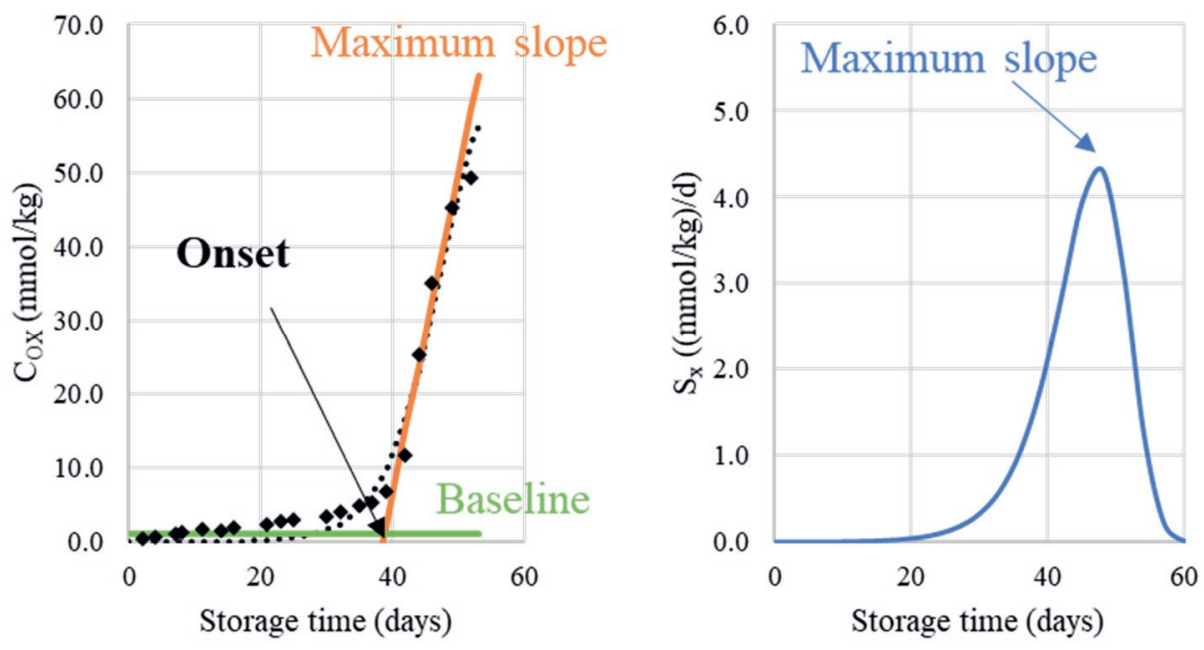
Figure S5.2. Statistical justification for the aldehyde onset prediction model. To verify whether it was justified to fit the linear regression of the prediction model through the origin, linear regression models with and without forcing the regression through the origin were compared to each other (Figure S5.5A/B). Both models fitted the data well, with comparable residual standard errors. No significant difference was found between the models by ANOVA analysis (Figure S5.7C). The residuals vs fitted plot (Figure S5.5D) showed a small trend, which did not worry us as we anticipated this due to using different formulations. The normal Q-Q plot (Figure S5.5E) showed that our data was normally distributed enough for this linear regression model.

\section{A}

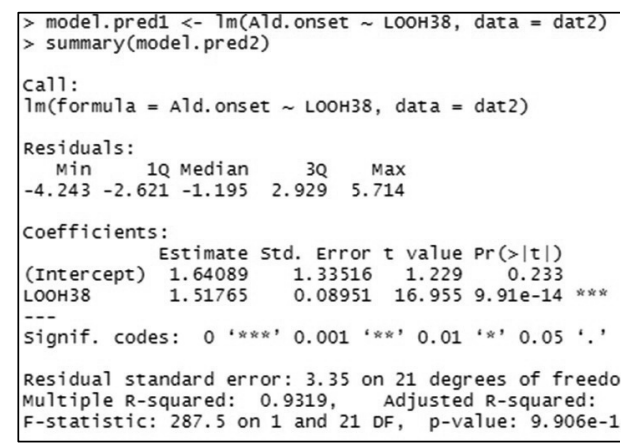

\section{C}

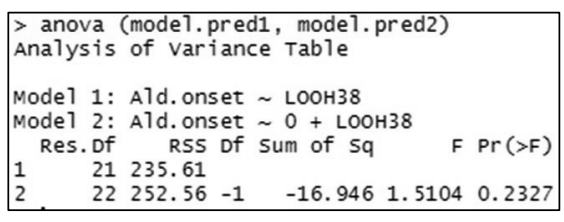

D

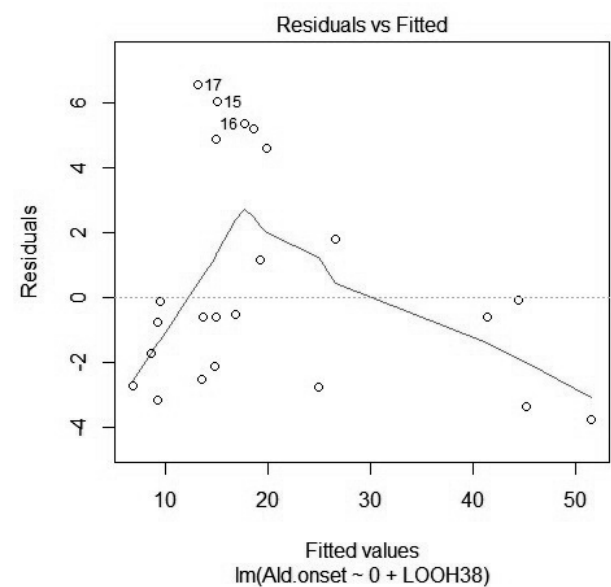

\section{B}
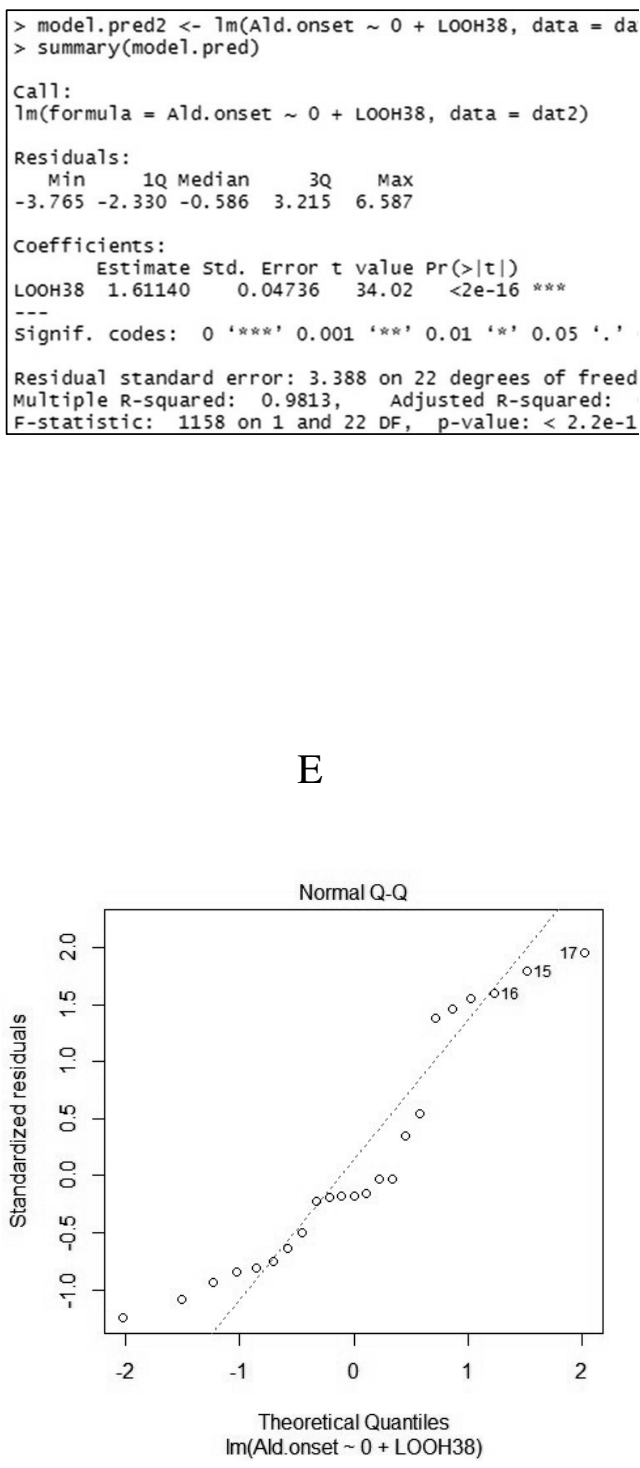
Figure S5.3. Reduced Foubert $(\mathrm{F} 3, \mathrm{n}=16.7)$ regression results on the Hydroperoxide data by ${ }^{1} \mathrm{H}$ NMR up to 40 $\mathrm{mmol} / \mathrm{kg}$. The dots are the actual measured data points, the solid line the F3 function and the dashed lines represent the $95 \% \mathrm{CI}$.
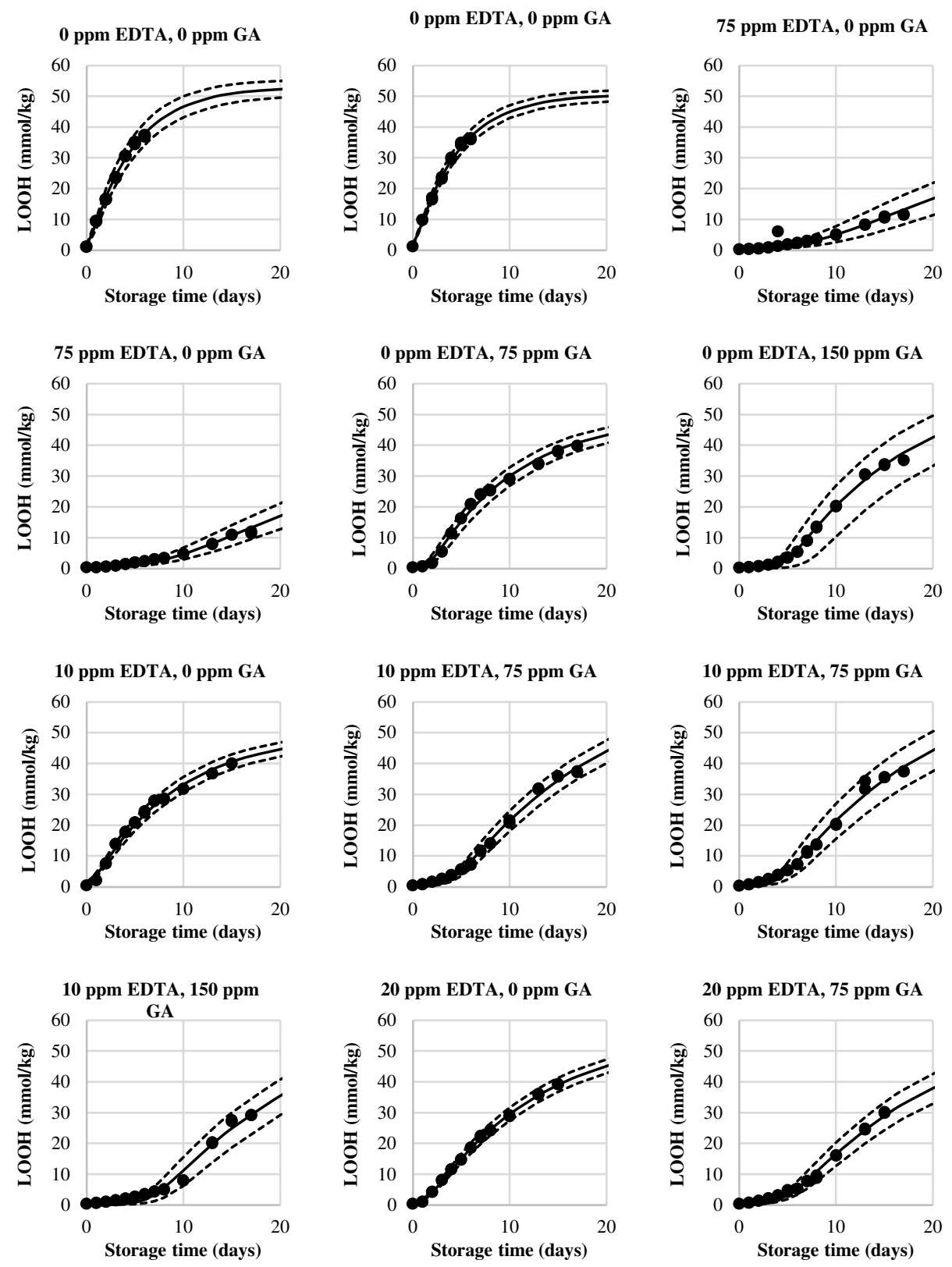


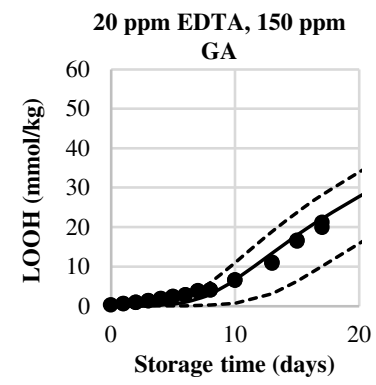

Figure S5.4. Reduced Foubert $(F 3, n=1.1)$ regression results on the Aldehyde data by ${ }^{1} \mathrm{H}$ NMR up to $20 \mathrm{mmol} / \mathrm{kg}$. The dots are the actual measured data points, the solid line the F3 function and the dashed lines represent the $95 \%$ CI.
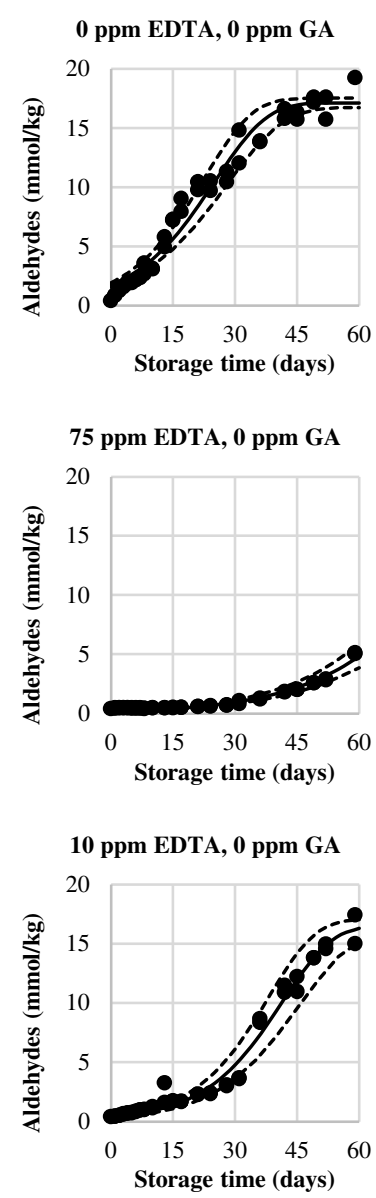
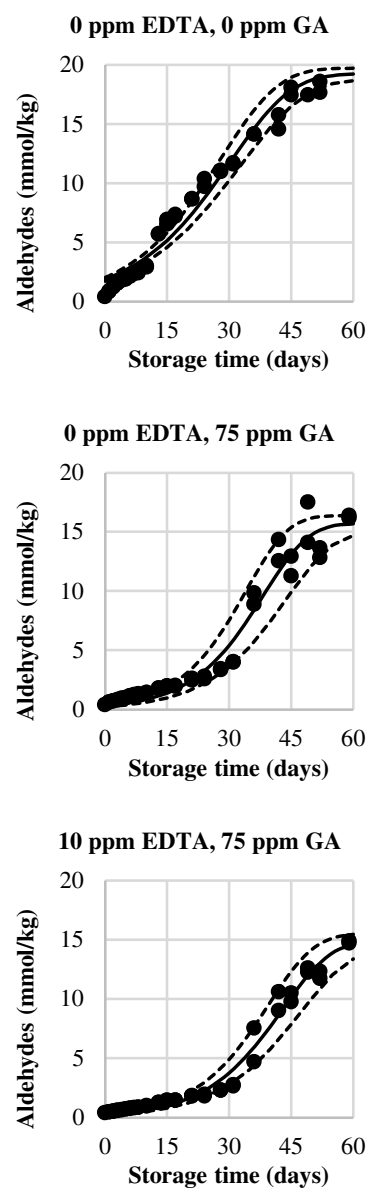

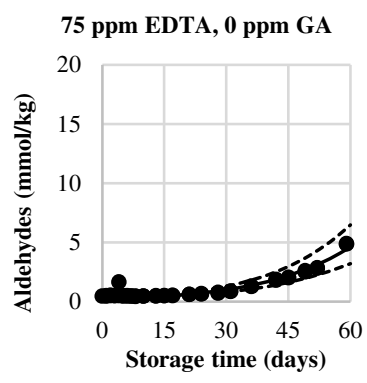

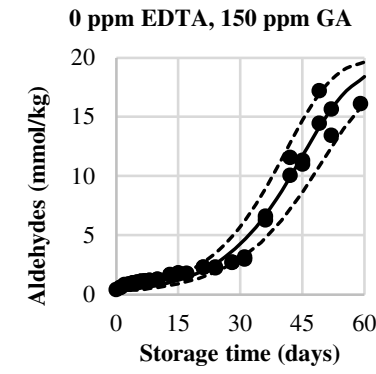

10 ppm EDTA, 75 ppm GA

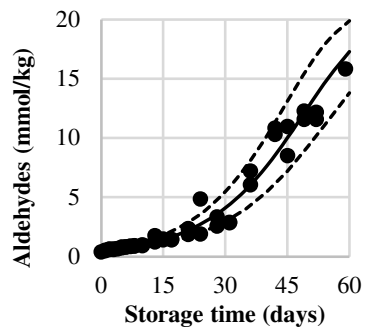



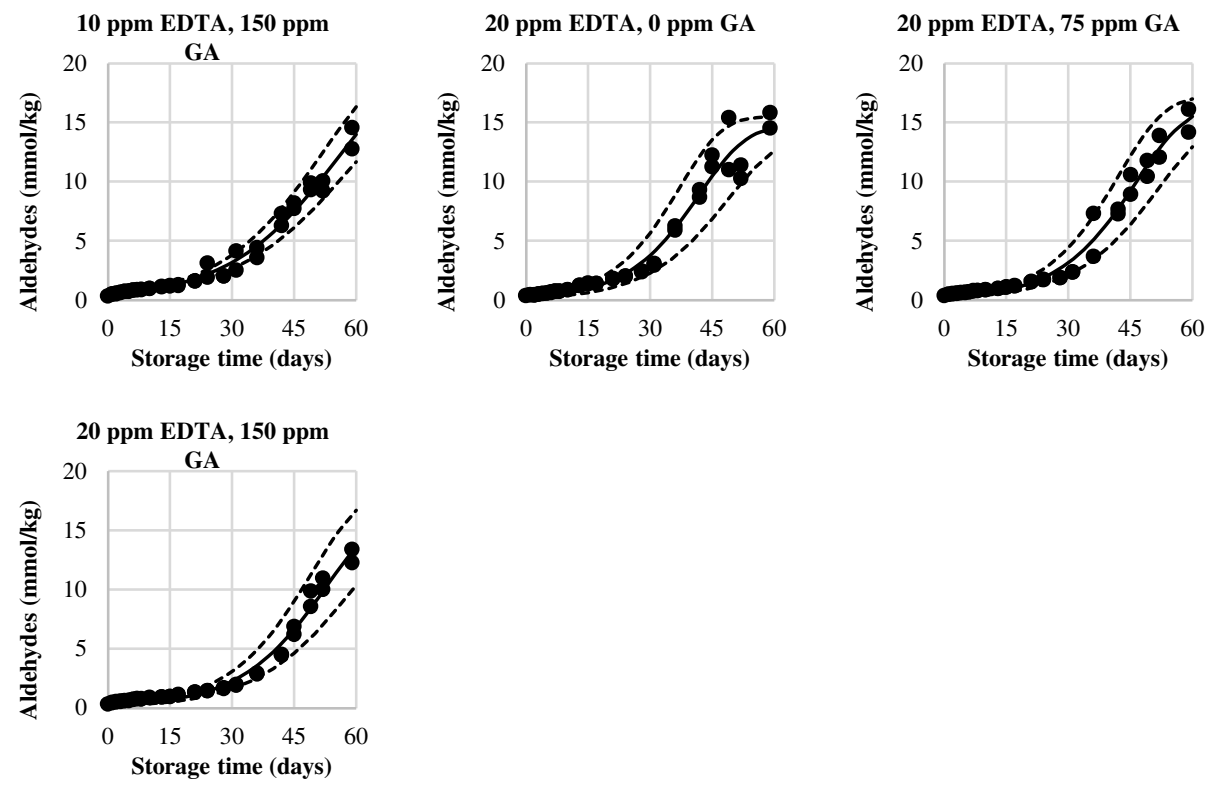
Figure S5.5. Overview of the parameters obtained from regression using the reduced Foubert (F3) function for the 'model validation' data set. A: f0, B: K. Shown error bars represent the $95 \%$ CI.

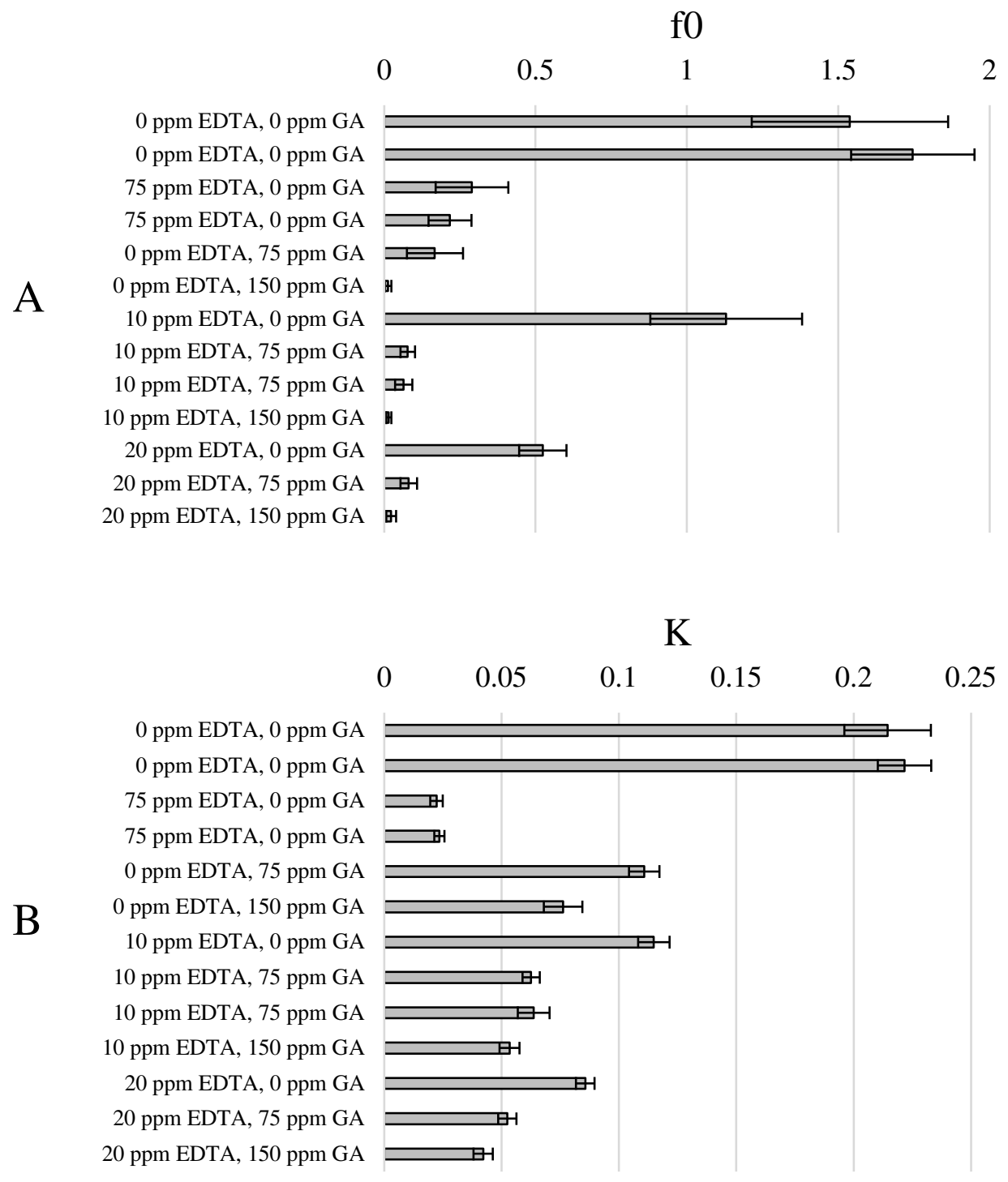




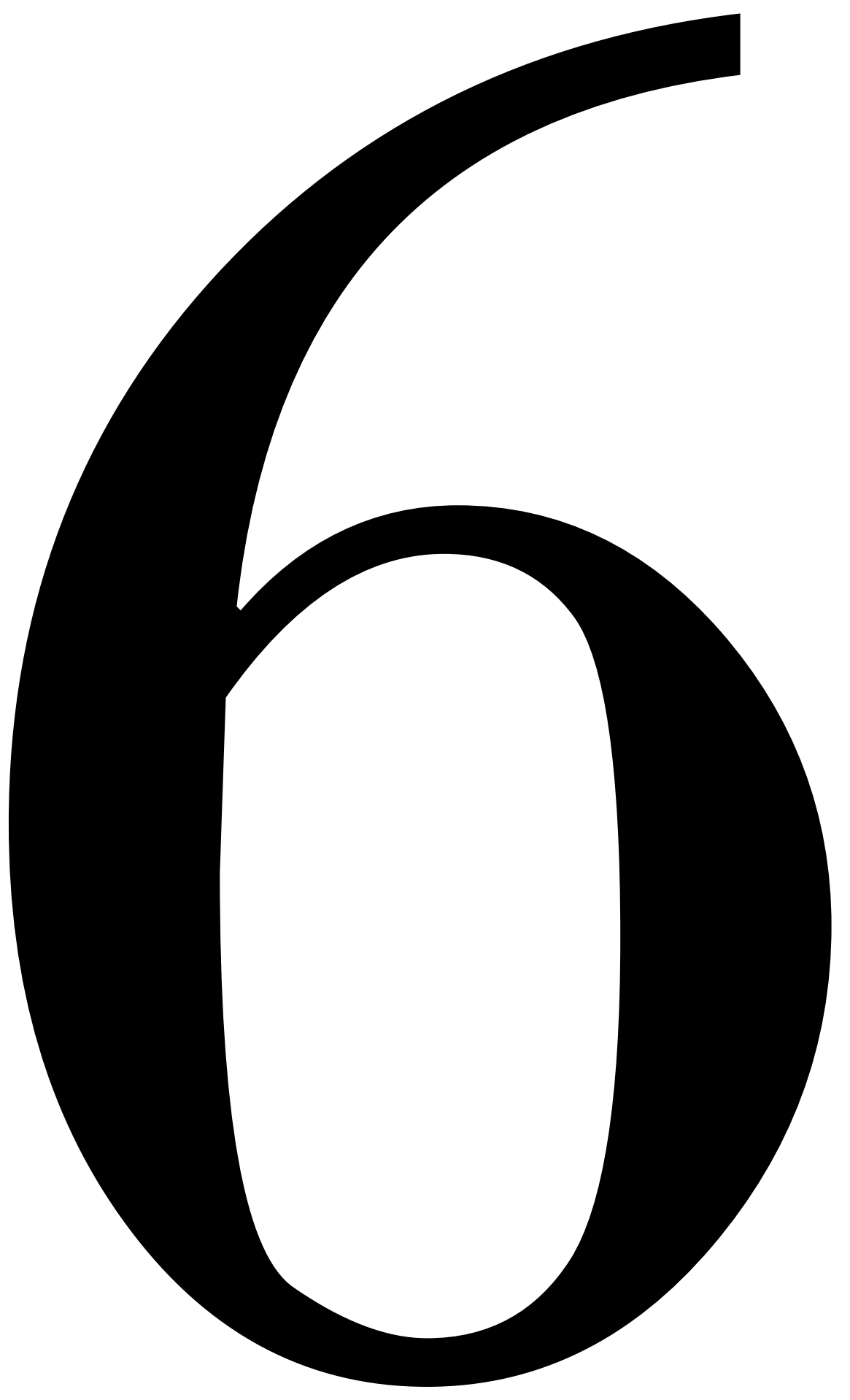




\section{Chapter 6}

\section{${ }^{31} \mathrm{P}$ NMR assessment of the phosvitin- iron complex in mayonnaise}

Based on: Merkx, D. W. H., Delić, F., Wierenga, P. A., Hennebelle, M., \& van Duynhoven, J. P. M. (2019). ${ }^{31}$ P NMR assessment of the phosvitin-iron complex in mayonnaise. Magnetic Resonance in Chemistry, 57(9), 540-547. 


\begin{abstract}
Lipid oxidation is the main reason for the limited shelf-life of mayonnaise. One of the main catalysts of this process is iron, which is introduced in its ferric (Fe(III)) form via phosvitin, an egg yolk phosphoprotein rich in phosphoserines. The binding of $\mathrm{Fe}(\mathrm{III})$ to phosvitin and its ability to establish a redox couple with Fe(II) is believed to determine the oxidation rate of unsaturated lipids. In this work, a ${ }^{31} \mathrm{P}$ NMR based method was developed to quantify loading of phosvitin with $\mathrm{Fe}(\mathrm{III})$ and its reductive release. Both features could be quantified in model phosvitin solutions by exploiting the paramagnetic broadening of ${ }^{31} \mathrm{P}$ NMR signal of phosphoserine residues by Fe(III). This method was then successfully applied to quantify the phosvitin-Fe(III) loading in mayonnaise water phase by liquid NMR, whereas ${ }^{31} \mathrm{P}$ NMR MAS could only provide a qualitative measure. The ${ }^{31} \mathrm{P}$ NMR method showed a direct relation between loading of the Fe(III)-phosvitin complex and lipid oxidation.
\end{abstract}




\subsection{Introduction}

In the western diet, one of the most consumed oil-in-water food emulsions is mayonnaise, which is rich in mono- and polyunsaturated fatty acids. A critical ingredient for physical stabilization of this emulsion is egg yolk (Anton, 2013; Anton \& Gandemer, 1997), which has a high content of surface-active phospholipids and proteins. The shelf-life of mayonnaise is compromised by oxidation of the unsaturated fatty acids that ultimately generate rancid off-flavours (Ghorbani Gorji, Smyth, Sharma, \& Fitzgerald, 2016). The enhanced oxidation of unsaturated lipids in mayonnaise has been attributed to the pro-oxidative catalytic role of iron (Frankel, 2005; Fukuzawa, Seko, Minami, \& Terao, 1993; Mei, Mcclements, Wu, \& Decker, 1998; Mozuraityte, Rustad, \& Storro, 2008). This involves the reaction of both Fe(II) and $\mathrm{Fe}(\mathrm{III})$ with lipid hydroperoxides, stimulating the free radical chain reaction (Frankel, 2005). In aqueous environments, the oxidation of Fe(II) goes extremely fast, allowing the $\mathrm{Fe}(\mathrm{III})$-catalysis to be rate determining (Mozuraityte et al., 2008). In mayonnaise, these iron ions are introduced via phosvitin, one of the most abundant (phospho)proteins in egg yolk (Anton, 2007). Phosvitin effectively binds iron in its ferric (Fe(III)) state (Samaraweera, Zhang, Lee, \& Ahn, 2011a). The binding capacity of phosvitin towards Fe(III) depends on several parameters such as $\mathrm{pH}$, salt concentration, phosphorylation degree of the phosphoserine residues and the presence of other metals (e.g., $\mathrm{Ca}^{2+}$ and $\mathrm{Mg}^{2+}$ ) (Anton, 2013; Castellani, Guérin-Dubiard, David-Briand, \& Anton, 2004; Grogan \& Taborsky, 1987; Taborsky, 1991). In hen egg yolk, where the $\mathrm{pH}$ is $6.5, \mathrm{Fe}(\mathrm{III})$ is strongly bound to phosvitin, forming the Fe(III)-phosvitin complex (Castellani et al., 2004). However, in mayonnaises, the $\mathrm{pH}$ is typically between 3.5 and 4.0, which significantly reduces binding strength for $\mathrm{Fe}(\mathrm{III})$ (Castellani et al., 2004). This weaker binding enables Fe(III) to start playing a significant role in lipid oxidation. An effective route to inhibit lipid oxidation in mayonnaise is the removal of iron from the Fe(III)-phosvitin complex by adding a strong chelator such as EDTA (Cho, Alamed, McClements, \& Decker, 2003), which has a higher binding strength to $\mathrm{Fe}(\mathrm{III})(\log \mathrm{K}=25)$ (Bates, Billups, \& Saltman, 1967) than phosvitin ( $\log \mathrm{K}=18-21$ ) (Hegenauer, Saltman, \& Nace, 1979). Other routes to inhibit lipid oxidation involve antioxidants that are hypothesised to either directly interact with the free-radical process or inhibit the activity of pro-oxidants (Ghorbani Gorji et al., 2016; Laguerre, Lecomte, \& Villeneuve, 2007).

No direct evidence for interactions of these anti-oxidants with the phosvitin-Fe(III) complex exists. To date, Fe(III)-phosvitin binding is assessed by incubating phosvitin solution with increasing amount of iron and measuring free iron remaining in the solution (Guérin-Dubiard, Anton, Dhene-Garcia, Martinet, \& Brulé, 2002). This approach is invasive, semi-quantitative 
and perturbing, which prompted us to explore the non-invasive and quantitative nature of ${ }^{31} \mathrm{P}$ NMR to provide a view on phosvitin-Fe(III) binding in model systems and mayonnaise.

Earlier work showed the ability of ${ }^{31} \mathrm{P}$ NMR to assess the effect of $\mathrm{pH}$ on phosvitin (Grogan, Shirazi, \& Taborsky, 1990; Vogel, 1983). Here, we want to expand on this approach by exploring the applicability of ${ }^{31} \mathrm{P}$ NMR for assessment of the Fe(III)-phosvitin binding. For this, the distinct magnetic properties of $\mathrm{Fe}(\mathrm{II})$ and $\mathrm{Fe}(\mathrm{III})$, which are respectively dia- and paramagnetic, will be exploited. Due to paramagnetic broadening, Fe(III) effectively quenches the ${ }^{31} \mathrm{P}$ NMR signal of bound phosphoserine residues in the phosvitin complex. In Figure 6.1 we outlined how this paramagnetic effect can be used for quantitative assessment of the amount of Fe(III) bound to phosvitin. Addition of a strong chelator, such as EDTA, removes paramagnetic $\mathrm{Fe}(\mathrm{III})$ from the vicinity of phosphoserine residues, increasing and sharpening their ${ }^{31} \mathrm{P}$ NMR signals (Levitt, 2008). Exploiting this feature, the amount of $\mathrm{Fe}(\mathrm{III})$ initially bound to phosvitin can be quantified. To assess the binding strength, the ${ }^{31} \mathrm{P}$ NMR approach can be used in combination with a reduction of Fe(III) and subsequent removal from the complex by $\mathrm{Fe}(\mathrm{II})$-specific chelation (Grogan \& Taborsky, 1987). In this study, the feasibility of these approaches was first established in model phosvitin solutions and subsequently applied in mayonnaise to investigate the binding of iron to phosvitin in regard to the oxidative stability of the lipid phase.

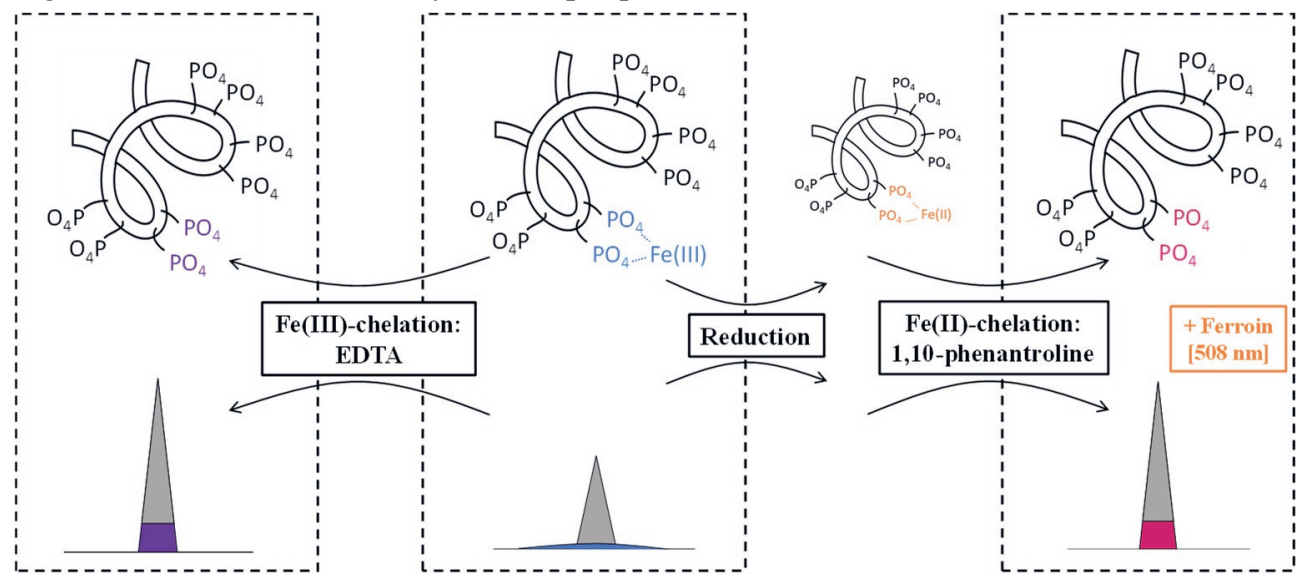

Figure 6.1. Schematic depiction of approaches used to assess loading of phosvitin with $\mathrm{Fe}(\mathrm{III})$ and its reductive release from the complex. On the top, the effects of chelation (left) and reductive release (right) on the $\mathrm{Fe}(\mathrm{III})$ phosvitin complex are depicted. On the bottom, the impact of these manipulations on the ${ }^{31} \mathrm{P}$ NMR-signal of phosvitin is shown. The grey integrals represent the free phosphoserines; the coloured parts represent the phosphoserines shown above (free and bound). Reductive release involves reduction of $\mathrm{Fe}$ (III) to $\mathrm{Fe}$ (II), which is specifically chelated by 1,10-phenanthroline. The resulting ferroin complex is detectable by UV/Vis at $508 \mathrm{~nm}$. 


\subsection{Material and Methods}

\subsubsection{Materials}

Deuterated water $\left(\mathrm{D}_{2} \mathrm{O}\right)$ was purchased from Euriso-top (Saint-Aubin, France). Phosvitin (isolated from hen egg yolk), 1,10-phenanthroline (PT), ethylenediaminetetraacetic acid (EDTA), trisodium trimetaphosphate (TMP), ferric chloride $\left(\mathrm{FeCl}_{3}\right)$ and ascorbic acid were all purchased from Sigma Aldrich (Zwijndrecht, the Netherlands). Ingredients for mayonnaise productions were purchased via local distributors (the Netherlands).

\subsubsection{Phosvitin model solutions experiments}

To prepare the model stock solution, $65 \mathrm{mg}$ purified phosvitin was dissolved directly in 10 $\mathrm{mL} \mathrm{D}_{2} \mathrm{O}$, after which the $\mathrm{pH}$ was adjusted to 4.5 with $10 \mathrm{mM} \mathrm{HCl}$. The actual phosphoserine molarity was determined for each prepared stock solution (according to Eq. 6.1 in the later 'Calculations' section). This way, we did not need to correct for the variance in purity of the phosvitin, nor its $\mathrm{Mw}$ (34 $\mathrm{kDa}^{2}$ on average) and phosphoserine residues content (123/216 on average) (Abe, Itoh, \& Adachi, 1982; Samaraweera, Zhang, Lee, \& Ahn, 2011b). For quantification with ${ }^{31} \mathrm{P}$ NMR, $250 \mu \mathrm{L}$ of TMP in $\mathrm{D}_{2} \mathrm{O}(15 \mathrm{mM})$ was added as an internal standard to $2 \mathrm{~mL}$ of the phosvitin stock solution.

For the assessment of the EDTA effect on the phosvitin ${ }^{31} \mathrm{P}$ NMR signal, $0.5 \mathrm{~mL}$ of an excess amount ( $>10 \mathrm{mM}$ ) of EDTA-solution in $\mathrm{H}_{2} \mathrm{O}$ was added to $2.5 \mathrm{~mL}$ of the phosvitin model solution. For the titration experiment with $\mathrm{Fe}(\mathrm{III}), 1.5 \mathrm{~mL}$ of phosvitin stock solution in $\mathrm{D}_{2} \mathrm{O}$ (7.2 mM phosphoserine) was added to 0.0-0.5 mL of $\mathrm{FeCl}_{3}$-solutions in $\mathrm{D}_{2} \mathrm{O}(7.2 \mathrm{mM}$ ) and filled up to $3 \mathrm{~mL}$ with $\mathrm{D}_{2} \mathrm{O}$, obtaining final concentrations ranging from 0.0 to $1.2 \mathrm{mM} \mathrm{Fe}$ (III) in steps of $0.2 \mathrm{mM}$.

\subsubsection{Mayonnaise production and storage}

Mayonnaises with a $78 \% \mathrm{w} / \mathrm{w}$ oil content were prepared using emulsification with a Silverson mixer (Papendrecht, the Netherlands). The final concentration of the water phase was $20.6 \%$ w/w. The main ingredients were egg yolk $(5 \% \mathrm{w} / \mathrm{w}), \mathrm{NaCl}(1.1 \% \mathrm{w} / \mathrm{w})$ and EDTA-solutions in $\mathrm{H}_{2} \mathrm{O}$. The EDTA-solutions were added to respectively obtain final concentrations of 0.0$4.8 \mathrm{mM}$ EDTA. Next, rapeseed oil $(78 \% \mathrm{w} / \mathrm{w})$ was slowly added to form the emulsion and finally spirit vinegar $(1.4 \% \mathrm{w} / \mathrm{w})$ to achieve the desired $\mathrm{pH}(3.8)$. For the oxidation study, two aliquots of $1 \mathrm{~g}$ mayonnaise per formulation were stored in $20 \mathrm{~mL}$ screwcap vials in the dark at $40^{\circ} \mathrm{C}$ for 5 days. The aliquots were then stored at $-20^{\circ} \mathrm{C}$ until further analysis. 


\subsubsection{Separation of the water phase of mayonnaise}

Fifteen grams of mayonnaise was transferred to a $20 \mathrm{~mL}$ Falcon tube. The samples were centrifuged at $20^{\circ} \mathrm{C}$ at $5186 \times \mathrm{g}$ for 60 minutes using a Sigma 6-16K Centrifuge (Shrewsbury, UK). On average, 1 to $2 \mathrm{~mL}$ of aqueous layer could be extracted per Falcon tube. For each sample, the aqueous layer from two Falcon tubes were pooled together. The extraction was performed by putting the Falcon tube upside down, and cutting off the bottom tip, allowing the aqueous layer to be pipetted of carefully. To $2 \mathrm{~mL}$ aqueous layer, $250 \mu \mathrm{L}$ TMP-solution in $\mathrm{D}_{2} \mathrm{O}(15 \mathrm{mM})$ was added as an internal standard. Samples were then transferred to a 10mm NMR tube.

\subsubsection{Ultraviolet-visible spectroscopy (UV-Vis)}

UV-Vis experiments were performed at room temperature on a Lambda $35 \mathrm{UV}-\mathrm{Vis}$ spectrophotometer by Perkin Elmer (Waltham, USA). Molar extinction coefficients at 508 $\mathrm{nm}$ were determined using a known solution of PT-Fe(II) and the Lambert-Beer equation. The Fe(III) reduction and subsequent Fe(II)-PT complexation was started by introducing 1 $\mathrm{mL}$ of phosvitin stock solution to $0.4 \mathrm{~mL}$ ascorbic acid in $\mathrm{H}_{2} \mathrm{O}(10 \mathrm{mM}), 0.4 \mathrm{~mL}$ PT in $\mathrm{H}_{2} \mathrm{O}$ $(10 \mathrm{mM})$, followed by addition of $\mathrm{NaCl}$ and $\mathrm{HCl}$ solutions to reach the desired $\mathrm{NaCl}-$ concentration ( $1 \%$ or $3 \% \mathrm{w} / \mathrm{w})$ and $\mathrm{pH}(3.5$ and 4.5$)$ respectively and a final volume of $4 \mathrm{~mL}$. The reduction was monitored over 6 hours. The time after which $80 \%$ of the Fe(III) was reduced, was called the $\mathrm{t}_{0.8}$-value.

\subsubsection{NMR acquisition and processing}

Model phosvitin solutions and the water layer of mayonnaise were measured with the following settings. ${ }^{31} \mathrm{P}$ NMR spectra were recorded on a Bruker Avance HD $700 \mathrm{MHz}$ (16.45 T) NMR spectrometer (Bruker BioSpin, Switzerland) equipped with a 10-mm BBO-probe. The internal temperature of the probe was set at $300 \mathrm{~K}$. A single pulse experiment (zg) was recorded with 128 scans, a relaxation delay of 10 seconds, an acquisition time of 1.7 seconds and a power of $70 \mathrm{~W}$. The $90^{\circ}$ pulse length was optimised and set at $23.35 \mu$ s for the model phosvitin-solutions and $43.75 \mu$ s for the water layer of mayonnaise. The receiver gain was set to the maximum value. The data was processed with Bruker TopSpin 3.2 software. Before Fourier transformation, an exponential window function with a line broadening factor of 1.0 $\mathrm{Hz}$ was applied, followed by manual phase correction and automatic baseline correction. The

${ }^{31} \mathrm{P}$ NMR spectra were further processed using MestReNova (Santiago de Compostela, Spain). Spectra were fitted (Figure S6.1) by selecting four regions, comprising of signals from phosvitin [ $\delta 0.4$ to $0.1 \mathrm{ppm}$ ], phospholipids [ $\delta 0.7$ to $0.3 \mathrm{ppm}$, tentative], free phosphate 
[ $\delta 0.12 \mathrm{ppm}$ ], and TMP [ $\delta$-21 to $-21.2 \mathrm{ppm}$, internal reference]. Each sample was recorded and processed in duplicate. For the whole mayonnaise sample, ${ }^{31} \mathrm{P}$ NMR Magic Angle Spinning experiments were performed on a Bruker Avance III $300 \mathrm{MHz}$ (7.05 T) (Bruker BioSpin, Switzerland) using $4 \mathrm{~mm}$ rotors, and an Agilent Inova $400 \mathrm{MHz}(9.4 \mathrm{~T})$ using $9 \mathrm{~mm}$ rotors. Single pulse experiments (zg) at a spinning speed of $1500 \mathrm{~Hz}$ were recorded, with a relaxation delay of 1 seconds and an acquisition time of 0.04 seconds.

For the quantification of hydroperoxides in mayonnaises that underwent the accelerated storage test $\left(5\right.$ days, $40^{\circ} \mathrm{C}$ ), the oil phase was first obtained by freeze-thawing of mayonnaise, which broke the emulsion. The separated oil phase was dissolved in a binary 5:1 $\mathrm{CDCl}_{3}$ :DMSO-d6 solvent. Band selective ${ }^{1} \mathrm{H}$ NMR spectra were then recorded on a $600 \mathrm{MHz}$ (14.1 T) Bruker Avance III NMR spectrometer (Bruker BioSpin, Switzerland) according to Chapter 2 (Merkx, Hong, Ermacora, \& van Duynhoven, 2018). The region between $\delta 13.0$ and $8.0 \mathrm{ppm}$ was selectively excited. The hydroperoxide signals that were quantified resonate between $\delta 11.2$ and $10.7 \mathrm{ppm}$. The calculations, including a factor that accounts for intensity loss during the selective pulse, are described in Chapter 2.

\subsubsection{Calculations}

In the model phosvitin solution and centrifuged mayonnaise water phase, the amount of free phosphoserine residues was expressed in $\mathrm{mM}$ according to the following equation:

$$
\mathrm{C}_{\text {PSer }}(\mathrm{mM})=\frac{1}{\mathrm{~V}_{\mathrm{Aq}}} \cdot \frac{\mathrm{W}_{\text {IS }}}{\mathrm{sV}_{\text {IS }}} \cdot \mathrm{f}_{\mathrm{IS}} \cdot \frac{\mathrm{I}_{\mathrm{PSer}}}{\mathrm{I}_{\mathrm{IS}}} \cdot \frac{\mathrm{N}_{\mathrm{IS}}}{\mathrm{N}_{\mathrm{PSer}}} \cdot \frac{\mathrm{P}_{\mathrm{IS}}}{\mathrm{MW}_{\mathrm{IS}}} \cdot 10^{3} \quad \text { Eq. } 6.1
$$

Here, $\mathrm{V}_{\mathrm{Aq}}$ is the amount of water in $\mathrm{mL}$ to which the internal standard solution was added. $\mathrm{W}$ is the weight of the solute in the stock solution in $\mathrm{mg}, \mathrm{sV}$ is the total amount of stock solution in $\mathrm{mg}, \mathrm{f}$ is the amount used of the stock solution to prepare the final NMR solution in $\mathrm{mg}$. I is the NMR signal intensity after signal integration, $\mathrm{N}$ is the number of nuclei and MW is the molecular weight, $\mathrm{P}$ is the purity in \%. Index Pvt stands for phosvitin, PSer for free phosphoserine and IS for internal standard. To validate the quantitative NMR approach, the repeatability and within-laboratory reproducibility was determined by measuring three samples every day for four days in duplicate (Miller \& Miller, 2010). The Fe(III)-phosvitin loading was determined by comparing the phosphoserine-content $\left(\mathrm{C}_{\mathrm{PSer}}\right)$ in a sample with and without an excess of EDTA $(>10 \mathrm{mM})$, assuming that the excess EDTA removes all $\mathrm{Fe}(\mathrm{III})$ from the phosvitin surface:

$$
\mathrm{Fe}(\mathrm{III})-\text { phosvitin loading }=\left(1-\frac{\mathrm{C}_{\mathrm{PSer}}[\text { no EDTA }]}{\mathrm{C}_{\mathrm{PSer}}[\text { with excess EDTA }]}\right) \cdot 100 \% \quad \text { Eq. } 6.2
$$




\subsection{Results and Discussion}

\subsection{1. ${ }^{31} \mathrm{P}$ NMR assessment of the Fe(III)-phosvitin binding in model solutions}

Model phosvitin stock solutions in $\mathrm{D}_{2} \mathrm{O}(6-10 \mathrm{mM}$ phosphoserine) were first qualitatively assessed using ${ }^{31} \mathrm{P}$ NMR at a magnetic field strength of $16.45 \mathrm{~T}$. In these ${ }^{31} \mathrm{P}$ NMR spectra, no signals of impurities were observed. As the phosphoserine residues in phosvitin had slightly different chemical and physical surroundings, they are presented as an envelope of signals that appear as an unresolved lineshape at pH 4 (Figure 6.2A) (Grogan et al., 1990; Vogel, 1983).
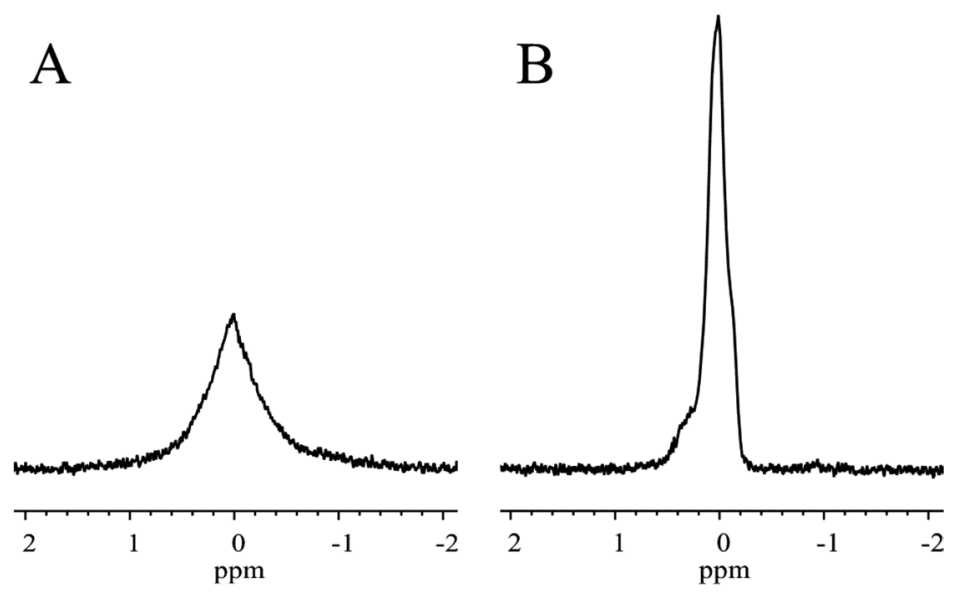

Figure 6.2. ${ }^{31} \mathrm{P}$ NMR spectra at $16.45 \mathrm{~T}$ of a model phosvitin solution in $\mathrm{D} 2 \mathrm{O}$ at $\sim \mathrm{pH} 4$ without (A) and with (B) removal of $\mathrm{Fe}(\mathrm{III})$ by an excess of EDTA. The samples were measured with identical acquisition and processing settings, plotted on the same scale.

To verify whether removal of the Fe(III) from the phosvitin complex indeed increased the signal due to the removal of the paramagnetic broadening, an excess of EDTA ( $>10 \mathrm{mM})$ was added to the solution. Both line-narrowing and increase in signal integral were observed upon addition of EDTA (Figure 6.2 B). We attributed the signal increase to the removal of the paramagnetic $\mathrm{Fe}(\mathrm{III})$ from phosvitin and used this effect for the quantification of the bound iron. We note that EDTA also removes other metal ions that can broaden ${ }^{31} \mathrm{P}$ NMR peaks, such as calcium and magnesium. This effect, however, does not broaden these signals beyond detection and does not interfere with the quantitative assessment of $\mathrm{Fe}$ (III) binding to phosvitin. The reproducibility of phosphoserine quantification by ${ }^{31} \mathrm{P}$ NMR was carried out according to validation procedures outlined by Miller and Miller (2010). In phosvitin solutions with phosphoserine concentrations in the range of 1.4-4.2 mM, we found a justified 
pooled relative standard deviation of both reproducibility and repeatability of $2 \%$, indicating that the method is robust both within-days and between-days.

To further investigate the quenching effect of $\mathrm{Fe}$ (III) and establish the stoichiometry of $\mathrm{Fe}$ (III) binding to phosphoserine residues of phosvitin, we titrated a model phosvitin solution with $\mathrm{FeCl}_{3}$ (Figure 6.3). As expected, the addition of $\mathrm{Fe}$ (III) decreased the intensity of the NMR signal significantly, because the signals of phosphoserine residues bound to paramagnetic $\mathrm{Fe}(\mathrm{III})$ ions are broadened beyond detection. It was found that one mole of $\mathrm{Fe}(\mathrm{III})$ quenches the ${ }^{31} \mathrm{P}$ NMR signal of $1.8 \pm 0.2$ moles of phosphoserine (Figure 6.3). This is in line with the binding stoichiometry of PSer/Fe(III) $=2 / 1$ reported in literature. After establishing the quenching stoichiometry, we found that $0.4 \mathrm{mM}$ of the $2.9 \mathrm{mM}$ total phosphoserines were bound to $\mathrm{Fe}(\mathrm{III})$, corresponding to $14 \% \mathrm{Fe}(\mathrm{III})$ loading.

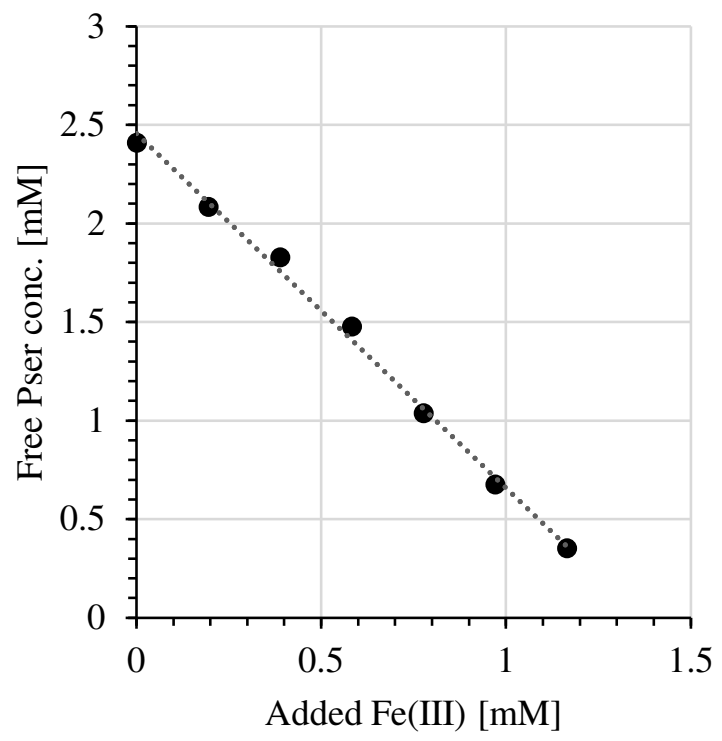

Figure 6.3. Titration of $\mathrm{Fe}(\mathrm{III})$ to a model phosvitin-solution. The free Pser concentration was quantified by ${ }^{31} \mathrm{P}$ NMR. The slope of the curve is PSer/Fe(III) $=1.8 \pm 0.2$.

The binding strength of $\mathrm{Fe}(\mathrm{III})$ to phosvitin was then assessed by observing the reductive release of this ion upon addition of ascorbic acid and 1,10-phenanthroline (PT), which respectively serve as reducer and $\mathrm{Fe}(\mathrm{II})$-specific chelator. We observed that over a span of four hours, over $95 \%$ of the $\mathrm{Fe}$ (III) was reduced to $\mathrm{Fe}$ (II) (Figure 6.4). The rate of reductive release at $\mathrm{pH} 3.5$ was not influenced by $\mathrm{NaCl}$-concentrations in the $1-3 \%$ range $\left(\mathrm{t}_{0.8}\right.$-values $=$ $24 \pm 1$ minutes). At $\mathrm{pH} 4.5$, the $\mathrm{t}_{0.8}$-values were 50 and 70 minutes at respectively $1 \%$ and $3 \% \mathrm{w} / \mathrm{w} \mathrm{NaCl}$. This is in line with earlier reports that show that lower $\mathrm{pH}$ and lower ionic strength weaken the phosvitin-iron complex significantly (Castellani et al., 2004). 


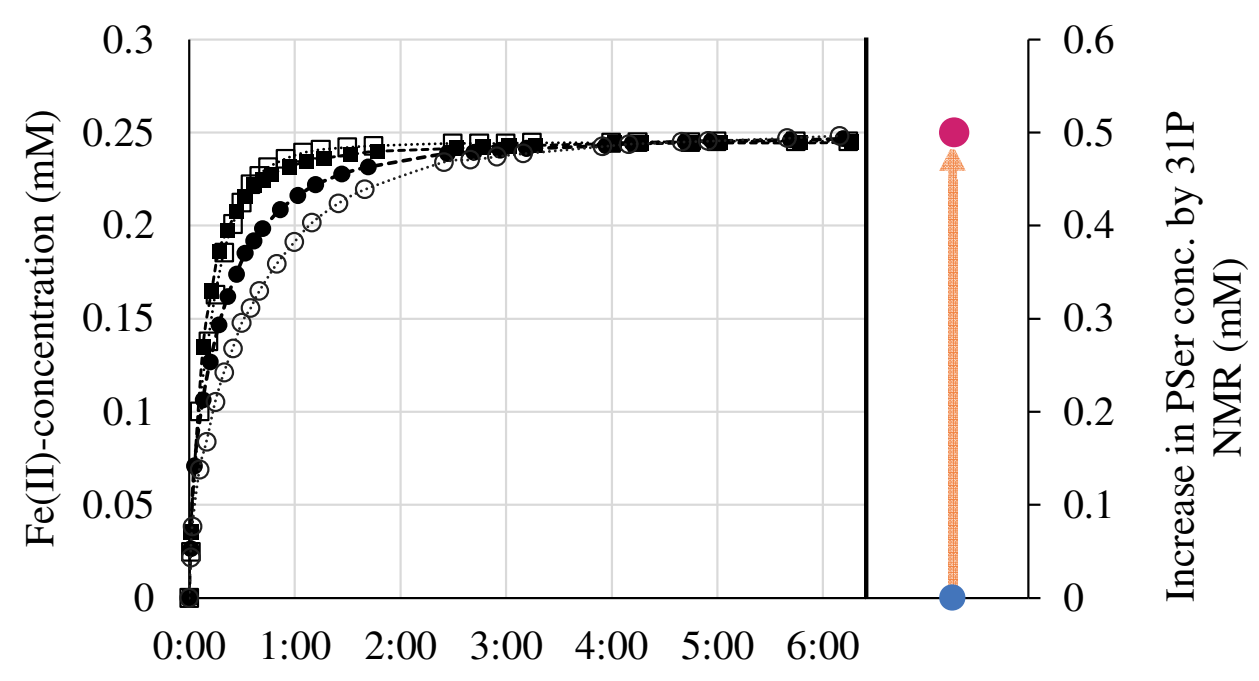

Reaction time (h:mm)

Figure 6.4. Kinetics of reductive release of Fe(III) from its complex with phosvitin, presented as concentration of the Fe(II)-PT (ferroin) complex upon addition of a reducer and PT. The left axis presents concentration of the ferroin complex due to reductive release in a model solution of phosvitin at ambient temperature: $\mathrm{pH} 4.5$ with $1 \% \mathrm{w} / \mathrm{w} \mathrm{NaCl}$ (•) and $3 \% \mathrm{w} / \mathrm{w} \mathrm{NaCl}(0)$, and $\mathrm{pH} 3.5$ with $1 \% \mathrm{NaCl}(\square)$ and $3 \%$ w/w $\mathrm{NaCl}(\square)$. On the right axis, the removal of the Fe(III), as measured by ${ }^{31} \mathrm{P}$ NMR is graphically represented, where (•) represents the pure phosvitin solution prior to reduction, and (๑) the net increase of the phosphoserine concentration after reduction (Figure 6.1).

Next, we assessed whether ${ }^{31} \mathrm{P}$ NMR can be used to assess reductive release of Fe(III) from its complex with phosvitin. We recorded ${ }^{31} \mathrm{P}$ NMR spectra before and after addition of PT and ascorbic acid and observed a signal increase, due to the removal of paramagnetic Fe(III) from the phosvitin complex. For all conditions, we observed that the concentration of chelated Fe(II) (left axis in Figure 6.4) was half of the increase of observable phosphoserine concentration by ${ }^{31} \mathrm{P}$ NMR (right axis in Figure 6.4), which was in line with the binding stoichiometry PSer/Fe(III) $=2 / 1$. This quantitative conversion also confirmed that the Fe(III) that is reduced to $\mathrm{Fe}$ (II) was initially bound in the Fe(III)-phosvitin complex and was not present in any other form in a significant amount. Moreover, the model solution experiments indicated that ${ }^{31} \mathrm{P}$ NMR can be used to assess the reductive release of Fe(III) from its complex with phosvitin in mayonnaise. The ${ }^{31} \mathrm{P}$ NMR method is less sensitive than UV-Vis and does not allow the monitoring of time-resolved kinetics of the reductive release, but it can be used for the assessment of the final state of this process. 


\subsection{2. ${ }^{31} \mathrm{P}$ NMR assessment of the Fe(III)-phosvitin binding in mayonnaise}

Now that the ${ }^{31} \mathrm{P}$ NMR method was validated in model systems, we explored its application in real mayonnaises. In full mayonnaise, the phosphoserine signals of phosvitin were poorly observable by liquid state ${ }^{31} \mathrm{P}$ NMR, due to signal broadening caused by the presence of magnetic susceptibility mismatches at oil/water interfaces. To narrow the ${ }^{31} \mathrm{P}$ NMR linewidths of phosvitin in full mayonnaise, we used semi-solid-state NMR, specifically Magic Angle Spinning (MAS). The ${ }^{31} \mathrm{P}$ MAS NMR spectra of mayonnaise (Figure 6.5) clearly showed a narrow signal of the phosphoserine residues of phosvitin, but also broader signals originating from phospholipids. The addition of excess EDTA $(>10 \mathrm{mM})$ did show a significant increase of the phosphoserine ${ }^{31} \mathrm{P}$ NMR signal intensity. This indicated that paramagnetic iron and other ions were effectively removed from phosvitin. To quantitatively assess iron loading on phosvitin using ${ }^{31} \mathrm{P}$ MAS NMR spectra, four mayonnaises were analysed with increasing amounts of EDTA (0, 0.1, 0.2 and $1.0 \mathrm{mM})$. We found, however, that the obtained signal-to-noise ratios were insufficient to quantify $\mathrm{Fe}$ (III)-phosvitin. This effect was attributed to the relatively low magnetic fields at which we performed these experiments (7.05-9.40 $\mathrm{T}$ ) and the high background of overlapping phospholipid signals. 

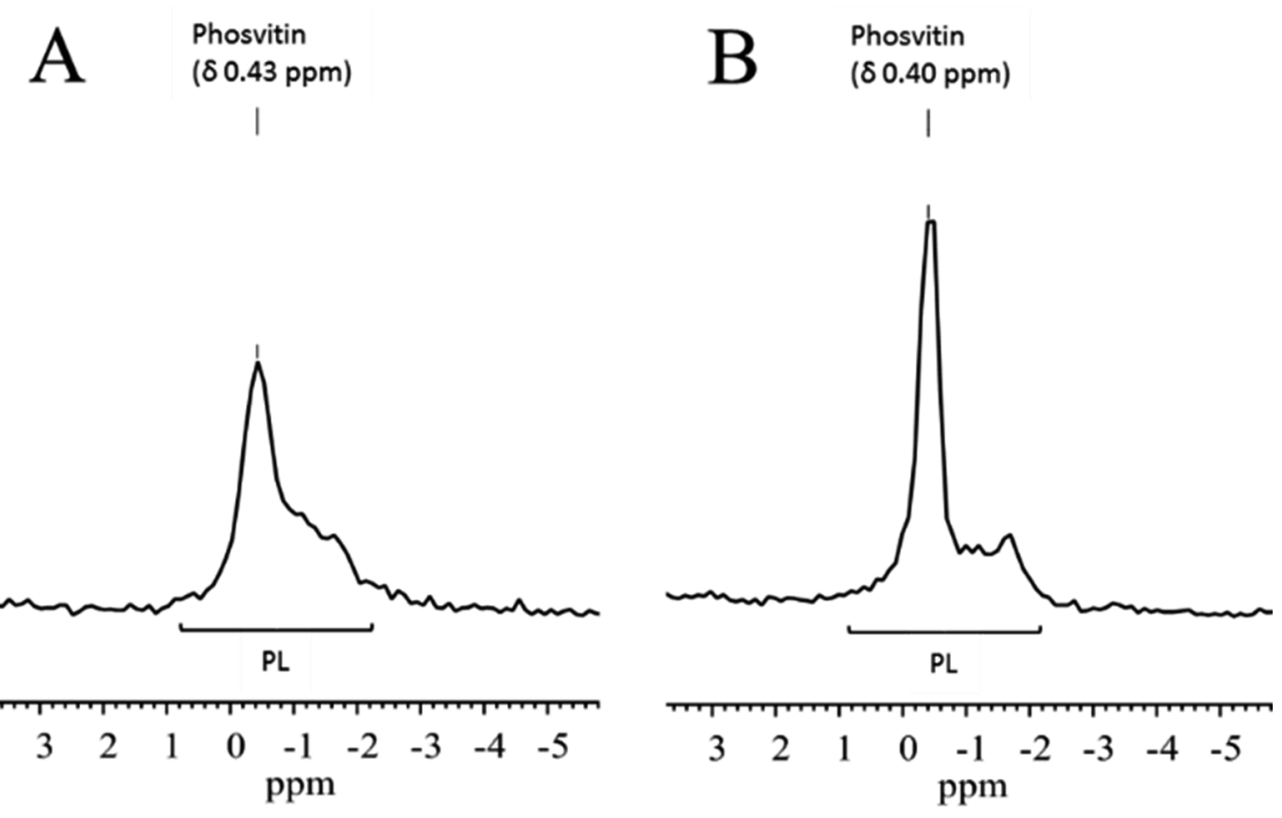

Figure 6.5. MAS ${ }^{31} \mathrm{P}$ NMR spectra at 7.05 $\mathrm{T}$ of mayonnaise formulated (A) without EDTA and (B) with an excess of EDTA $(>10 \mathrm{mM})$. The peak at $\delta-0.40 / 0.43$ ppm was assigned to phosvitin, the broad envelope of peaks beneath was tentatively assigned to phospholipids (PL). The samples were measured with identical acquisition and processing settings, plotted on the same scale.

Since ${ }^{31} \mathrm{P}$ MAS NMR spectra only allowed for qualitative assessment of Fe(III)-phosvitin binding and full mayonnaise could not be analysed by liquid-state NMR, the mayonnaise water layer was separated by centrifugation. This water layer was expected to have a composition similar to the continuous aqueous phase of this emulsion, as reported in literature. Liquid ${ }^{31} \mathrm{P}$ NMR spectra of the water layer indeed showed well resolved and narrow resonances which allowed for quantification of PSer concentrations, using similar settings as for the model phosvitin solution (Figure S6.1). The PSer concentration was determined in the water phase before emulsification and compared to the PSer concentration in the water phase after centrifugation. Here, we found that $90-95 \%$ of the dosed phosvitin was recovered in the water phase of mayonnaise. The remaining fraction of the phosvitin $(<10 \%)$ was expected to be present at the droplet interface, which is in line with earlier findings (Aluko \& Mine, 1997).

To further investigate the Fe(III)-phosvitin binding in the aqueous phase, we monitored the reductive release in the water layer of mayonnaise by ${ }^{31} \mathrm{P}$ NMR. Upon addition of PT, both with and without ascorbic acid, the linewidth of the phosvitin ${ }^{31} \mathrm{P}$ NMR peak narrowed, but did not show a significant integral increase (Figure 6). The lack of signal increase was 
believed to be due to the strong oxidative environment which maintains iron in its $\mathrm{Fe}$ (III) form and thus impedes reductive release in mayonnaise. The narrowing effect was attributed to removal of bivalent cations such as calcium that are known to complex with phosphoserines, inducing aspecific broadening. We proceeded by using the ${ }^{31} \mathrm{P}$ NMR method to assess the loading of the Fe(III)-phosvitin complex in the water phase of mayonnaise in relation to oxidative stability.

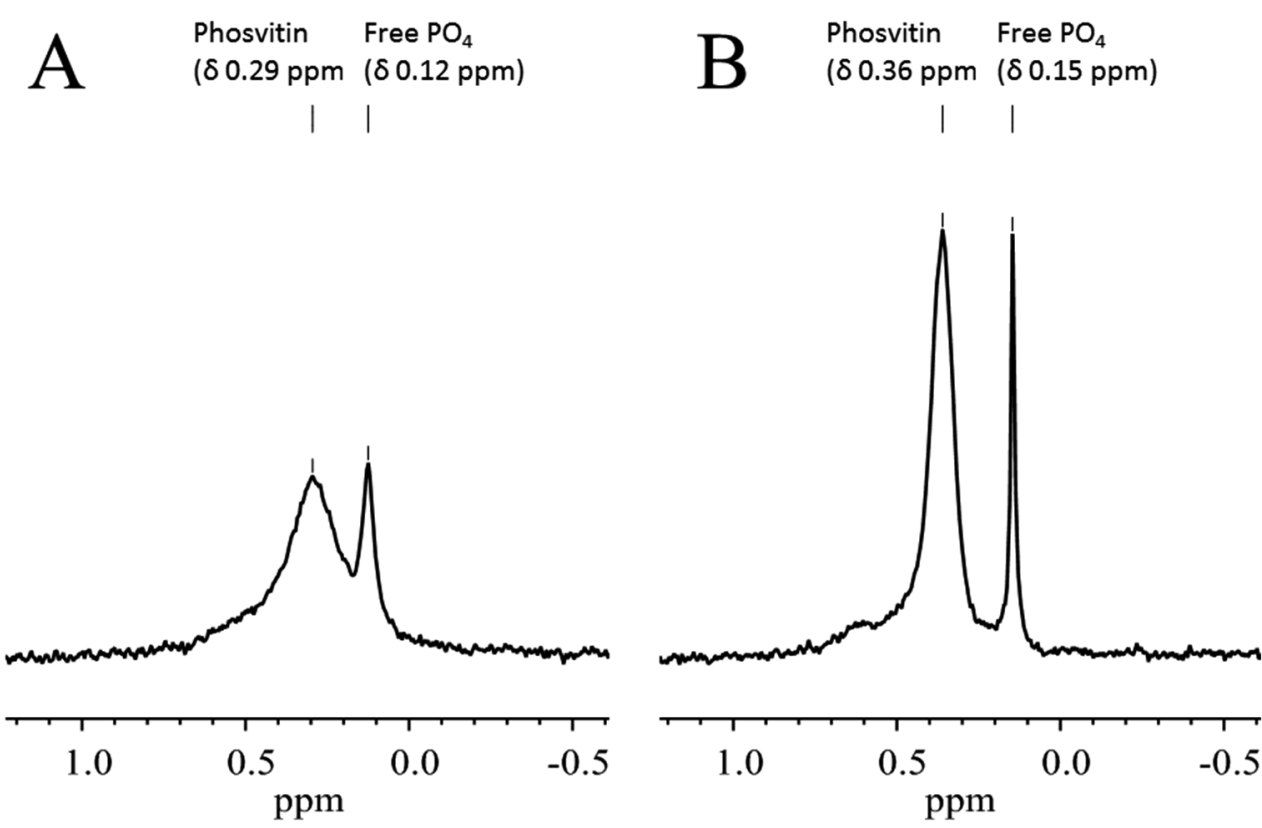

Figure 6.6. Liquid ${ }^{31} \mathrm{P}$ NMR spectra (16.45 T) of the water phase of a mayonnaise (A) without reducer and (B) with addition of PT and ascorbic acid. This ppm-range does not show TMP. The linewidth changes significantly, but no significant effect on integral was observed. The samples were measured with identical acquisition and processing settings, plotted on the same scale.

We studied the effects of Fe(III) removal from the phosvitin complex in mayonnaise by EDTA-chelation. Concentrations up to the stoichiometric point of EDTA to iron $(0.2 \mathrm{mM})$ and the typical level in commercial formulations $(0.8 \mathrm{mM})$ were tested. This EDTA-addition resulted in an increase of the ${ }^{31} \mathrm{P}$ NMR signal intensity and an overall line sharpening (Figure 6.7) with higher EDTA-concentrations. 


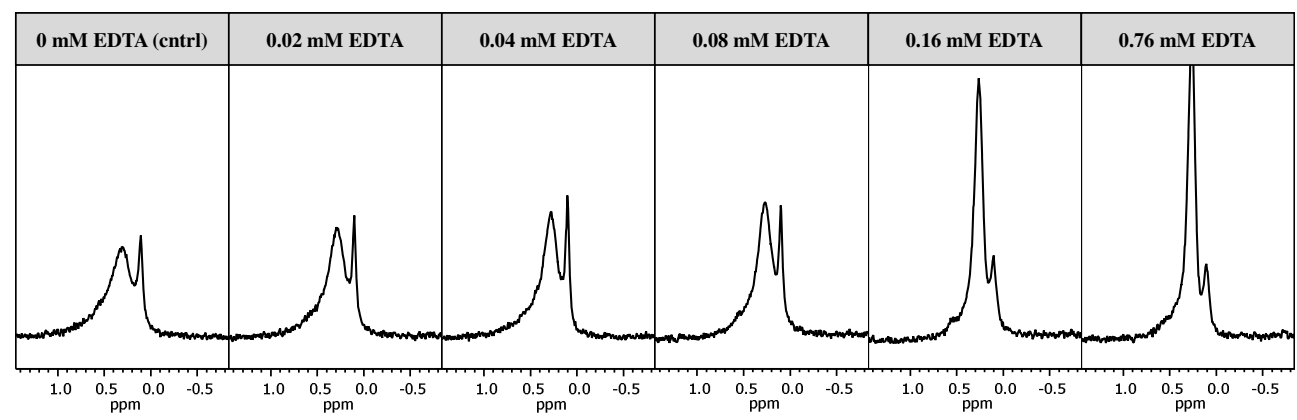

Figure 6.7. ${ }^{31} \mathrm{P}$ NMR spectra (16.45 T) of water phase of mayonnaise with a range of added EDTA levels added before emulsification. The samples were measured with identical acquisition and processing settings, plotted on the same scale. Assignment of the peaks are depicted in Figure 6.6. Peak areas of the phosvitin peaks were used to calculate the Fe(III)-loading presented on Figure 6.8, according to Eq 6.1 and 6.2.

From the ${ }^{31} \mathrm{P}$ NMR signal intensities, we calculated the Fe(III)-loadings of phosvitin, which showed a decreasing loading with EDTA addition (Figure 8). Hydroperoxide generation, used as measure to assess oxidative stability, decreased upon addition of EDTA (0-0.8 mM) (Figure 6.8). The removal of Fe(III) from the Fe(III)-phosvitin complex by EDTA had a significant effect on the oxidative stability of mayonnaise, as shown by the lower hydroperoxide concentrations observed after 5 days of storage.

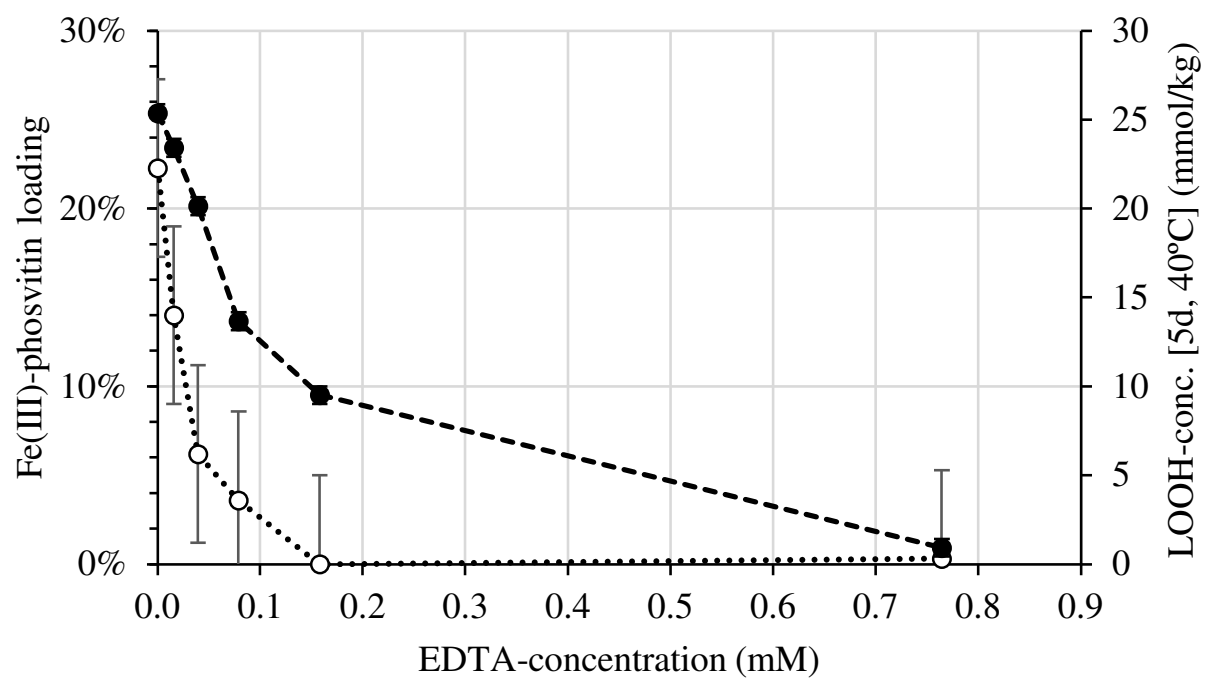

Figure 6.8. Relation between EDTA-concentration and the phosvitin-Fe(III) loading determined by ${ }^{31} \mathrm{P}$ NMR (left axis, O), and lipid peroxidation, expressed as the hydroperoxide ( $\mathrm{LOOH}$ ) concentration after 5 days storage at $40^{\circ} \mathrm{C}$ (right axis, $\bullet$ ). Dotted lines are intended to guide the eye. 
With EDTA-chelation, Fe(III) was removed from the phosvitin-complex, and the lipid oxidation was inhibited. Concentrations of EDTA above or equal to $0.2 \mathrm{mM}$ removed all $\mathrm{Fe}$ (III) from the complex in the water layer, but only an excess of EDTA $(0.8 \mathrm{mM})$ fully inhibited lipid oxidation. This increase of oxidative stability due to an EDTA excess might indicate that the iron is more effectively removed from the interface area or that also other pro-oxidant metal ions are chelated. With the presented ${ }^{31} \mathrm{P}$ NMR method, further research can be pursued on the relation between Fe(III) loading of phosvitin and oxidative stability of mayonnaise, particularly with alternative antioxidant systems.

\subsection{Conclusion}

For the first time it has been demonstrated that the Fe(III)-phosvitin loading can be directly assessed in a quantitative and non-invasive manner by ${ }^{31} \mathrm{P}$ NMR. This method exploits the effect of paramagnetic Fe(III) on the ${ }^{31} \mathrm{P}$ NMR signal, allowing a quantitative assessment of the complex in a model phosvitin solutions and in the water phase of mayonnaise. In the full mayonnaise, the ${ }^{31} \mathrm{P}$ NMR signals of phosvitin can be observed by MAS, but this only allows for qualitative assessment of the Fe(III) binding. Lowering the Fe(III) loading of phosvitin in mayonnaise by EDTA-chelation correlates well with stability against lipid oxidation.

\subsection{Literature}

Abe, Y., Itoh, T., \& Adachi, S. (1982). Fractionation and Characterization of Hen's Egg Yolk Phosvitin. Journal of Food Science, 47(6), 1903-1907.

Aluko, R. E., \& Mine, Y. (1997). Competitive Adsorption of Hen's Egg Yolk Granule Lipoproteins and Phosvitin in Oil-in-Water Emulsions. Journal of Agricultural and Food Chemistry, 45, 4565-4570.

Anton, M. (2007). Phosvitin. In Bioactive Egg Compounds. Germany: Springer.

Anton, M. (2013). Egg yolk: structures, functionalities and processes. J Sci Food Agric, 93(12), 2871-2880.

Anton, M., \& Gandemer, G. (1997). Composition, Solubility and Emulsifying Properties of Granules and Plasma of Egg Yolk. Journal of Food Science, 62(3), 484-487.

Bates, G. W., Billups, C., \& Saltman, P. (1967). The Kinetics and Mechanism of Iron(III) Exchange between Chelates and Transferrin. Journal of Biological Chemistry, 242(12), 2816-2821.

Castellani, O., Guérin-Dubiard, C., David-Briand, E., \& Anton, M. (2004). Influence of physicochemical conditions and technological treatments on the iron binding capacity of egg yolk phosvitin. Food Chemistry, 85(4), 569-577.

Cho, Y.-J., Alamed, J., McClements, D. J., \& Decker, E. A. (2003). Ability of Chelators to Alter the Physical Location and Prooxidant Activity of Iron in Oil-in-Water Emulsions. Journal of Food Science, 68(6), 1952-1957.

Frankel, E. N. (2005). Free radical oxidation. In Lipid Oxidation (2nd ed., pp. 15-24): Elsevier. 
Fukuzawa, K., Seko, T., Minami, K., \& Terao, J. (1993). Dynamics of iron-ascorbate-induced lipid peroxidation in charged and uncharged phospholipid vesicles. Lipids, 28(6), 497-503.

Ghorbani Gorji, S., Smyth, H. E., Sharma, M., \& Fitzgerald, M. (2016). Lipid oxidation in mayonnaise and the role of natural antioxidants: A review. Trends in Food Science \& Technology, 56, 88-102.

Grogan, J., Shirazi, A., \& Taborsky, G. (1990). Phosphorus Nuclear Magnetic Resonance of Diverse Phosvitin Species. Comparitive Biochemistry and Physiology, 96B(4), 655663.

Grogan, J., \& Taborsky, G. (1987). Iron binding by phosvitin: variation of rate of iron release as a function of the degree of saturation of iron binding sites. Journal of Inorganic Biochemistry, 29, 33-47.

Guérin-Dubiard, C., Anton, M., Dhene-Garcia, A., Martinet, V., \& Brulé, G. (2002). Hen egg and fish egg phosvitins: composition and iron binding properties. European Food Research and Technology, 214(6), 460-464.

Hegenauer, J., Saltman, P., \& Nace, G. (1979). Iron(III)-Phosphoprotein Chelates: Stoichiometric Equilibrium Constant for Interaction of Iron(III) and Phosphorylserine Residues of Phosvitin and Casein. Biochemistry, 18(18), 38653879.

Laguerre, M., Lecomte, J., \& Villeneuve, P. (2007). Evaluation of the ability of antioxidants to counteract lipid oxidation: existing methods, new trends and challenges. Progress in Lipid Research, 46(5), 244-282.

Levitt, M. H. (2008). Spin Dynamics (2nd ed.). Chichester: Wiley and Sons.

Mei, L., Mcclements, D. J., Wu, J., \& Decker, E. A. (1998). Iron-catalyzed lipid oxidation in emulsion as affected by surfactant, $\mathrm{pH}$ and $\mathrm{NaCl}$. Food Chemistry, 61(3), 307-312.

Merkx, D. W. H., Hong, G. T. S., Ermacora, A., \& van Duynhoven, J. P. M. (2018). Rapid Quantitative Profiling of Lipid Oxidation Products in a Food Emulsion by (1)H NMR. Anal Chem, 90(7), 4863-4870.

Miller, J. N., \& Miller, J. C. (2010). Statistics and Chemometrics for Analytical Chemistry (6th ed.): Pearson Education Limited.

Mozuraityte, R., Rustad, T., \& Storro, I. (2008). The Role of Iron in Peroxidation of Polyunsaturated Fatty Acids in Liposomes. Journal of Agricultural and Food Chemistry, 56, 537-543.

Samaraweera, H., Zhang, W. G., Lee, E. J., \& Ahn, D. U. (2011a). Egg Yolk Phosvitin and Functional Phosphopeptides-Review. Journal of Food Science, 76(7), R143-150.

Samaraweera, H., Zhang, W. G., Lee, E. J., \& Ahn, D. U. (2011b). Egg Yolk Phosvitin and Functional Phosphopeptides-Review. Journal of Food Science, 76(7), R143R150.

Taborsky, G. (1991). On the Interaction of Phosvitins With Ferric Ion: Solubility of the Fe(III)-Phosphoprotein Complex Under Acidic Conditions is a Function of the Iron Phosphate Ratio and the Degree of Phosvitin Phosphorylation. Journal of Inorganic Chemistry, 44, 65-77.

Vogel, H. J. (1983). Structure of Hen Phosvitin: A 31P NMR, 'H NMR, and Laser Photochemically Induced Dynamic Nuclear Polarization $H$ NMR Study. Biochemistry, 22, 668-674. 


\subsection{Supporting information}

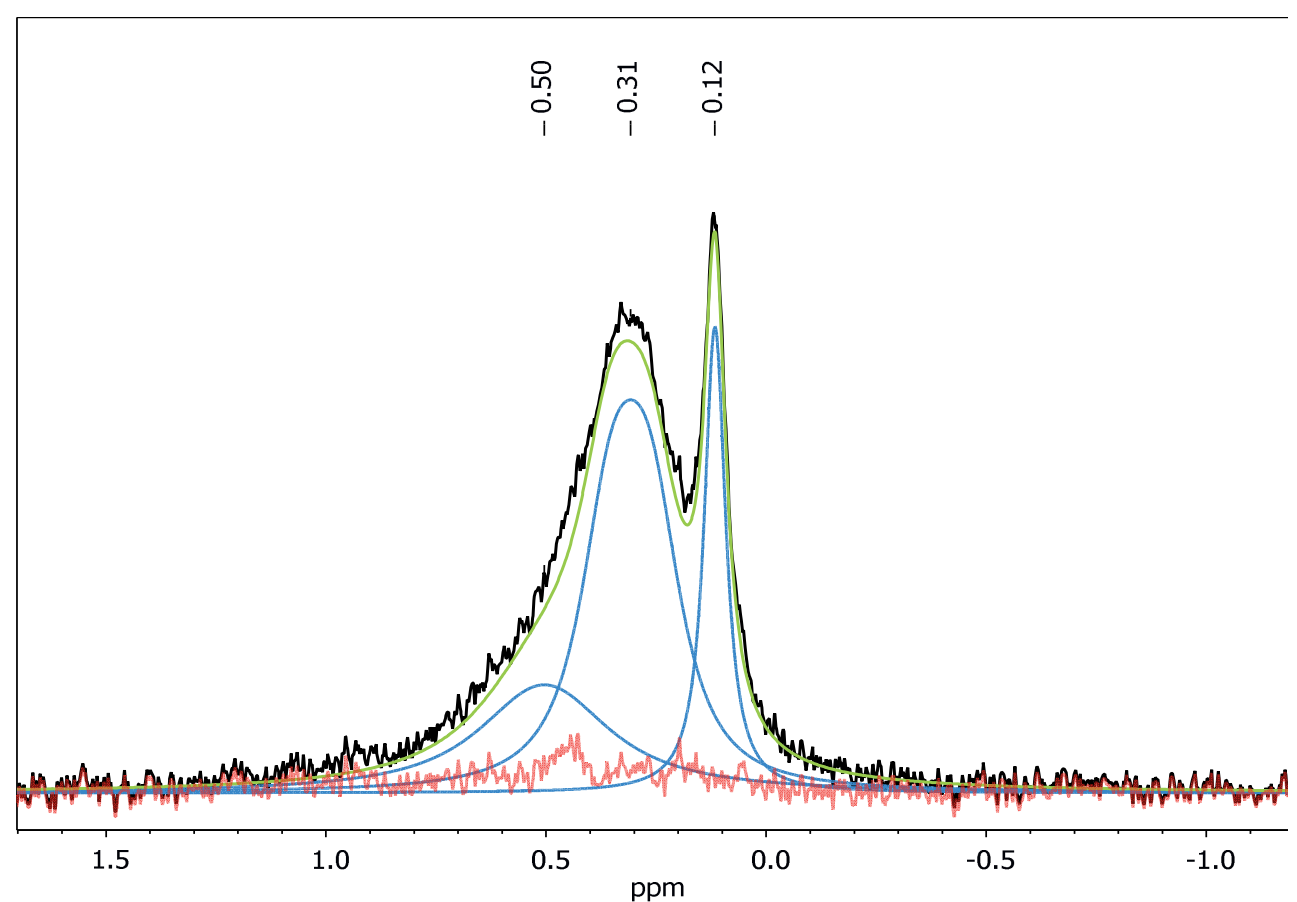

Figure S6.1. Example of line fitting on the ${ }^{31} \mathrm{P}$ NMR signal of the water phase of mayonnaise. The black line is the recorded ${ }^{31} \mathrm{P}$ NMR spectrum, the blue lines are the fits with a flexible ratio of Lorentzian/Gaussian functions, the green line is the summation and red the residual. The signals from left to right: tentatively assigned phospholipids (PL) at $\delta 0.50 \mathrm{ppm}$, phosvitin at $\delta 0.31 \mathrm{ppm}$ and free phosphate at $\delta 0.12 \mathrm{ppm}$. The displayed ppm-range does not show TMP ( $\delta-21.0$ to $21.2 \mathrm{ppm})$. 


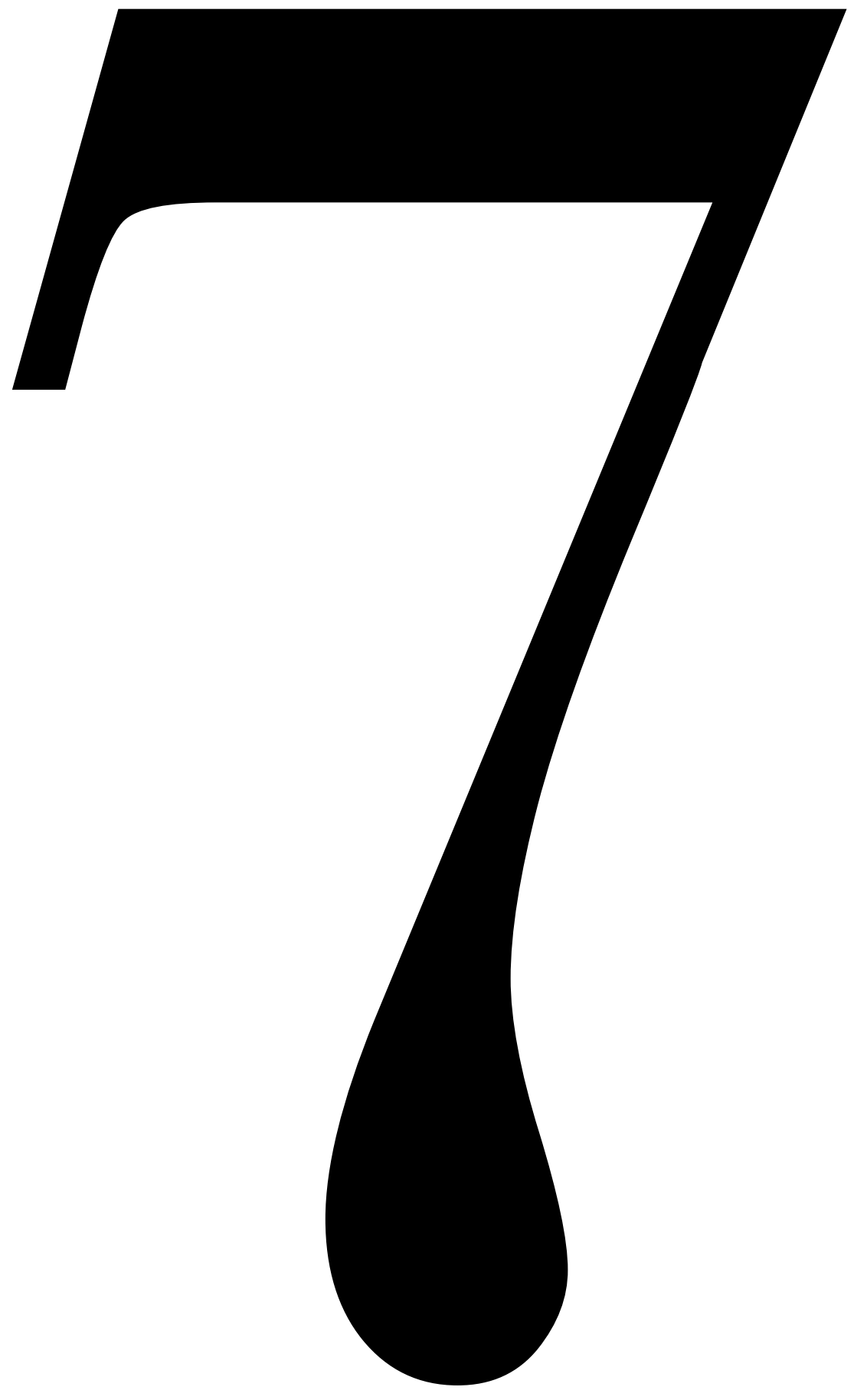


Chapter 7

General Discussion 


\subsection{Main findings and outline of the discussion}

The aim of this thesis was to discover mechanism-based early markers for lipid oxidation in (accelerated) storage of food emulsions (Figure 7.1). The mechanistic underpinning of these markers should also allow the discrimination of the involved dominant pathways. Hereto, we developed quantitative NMR and ESR methods that cover major lipid oxidation initiators and intermediates. In Chapter 2, and in more detail in the addendum of that chapter, a qNMR method was presented for an accurate and quantitative assessment of both hydroperoxides and aldehydes. In Chapter 3, we added to this another NMR-method for the assessment of epoxides in food emulsions and oils. In Chapter 4, radical intermediates were successfully assessed with ESR, and we shed a light on the interpretation of spin-trap application for early assessment of oxidative stability. In Chapter 5, we used the early oxidation products to train a prediction model for late oxidation kinetics. Traditional shelf-life test cycles of weeks can be shortened significantly by using the hydroperoxides rather than the aldehydes themselves for the assessment of oxidative stability. In Chapter 6, we showed that ${ }^{31} \mathrm{P}$ NMR could be used to specifically investigate the phosvitin-iron complexation, a critical aspect of lipid oxidation catalysis in mayonnaise.

In this Chapter, the insights and implications from the previous chapters are discussed and put into broader perspective. First, the strengths and limitations of the presented methods, both spectroscopic and modelling, will be reflected upon. Next, we will discuss how mechanistical markers enable the mechanistic interpretation of accelerated shelf-life tests, and the pivotal role of hydroperoxides in mayonnaise. The wider applicability of the provided methods will be discussed including other oil types and matrices. Lastly, future perspectives on prediction of sensorial properties and means to further elucidation of the physico-chemical interplay in emulsions are outlined. 


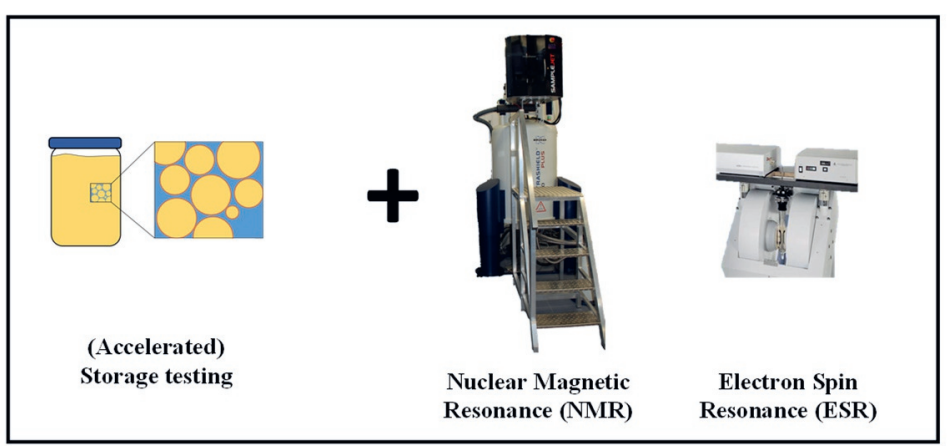

Magnetic resonance is a powerful tool to

identify and quantify major oxidation

products (Chapter 2, Chapter 3, Chapter 4)

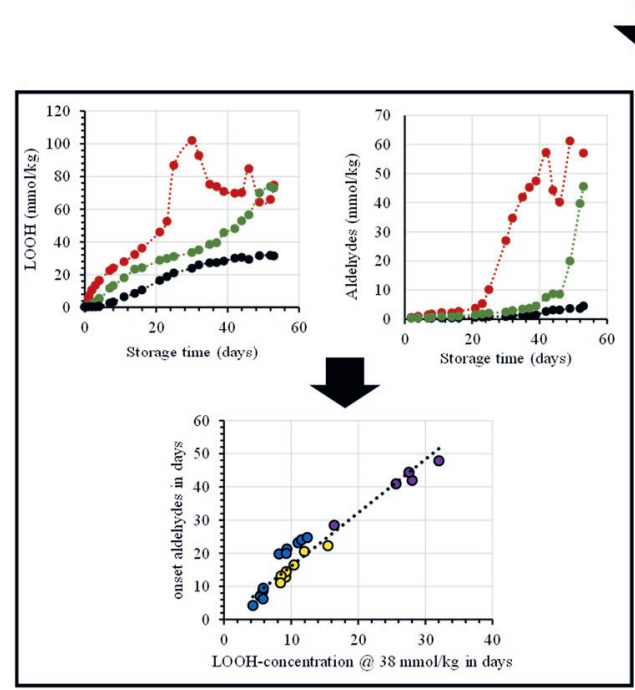

Successful prediction of aldehyde onset based on semi-empirical LOOH modeling (Chapter 2, Chapter 5)
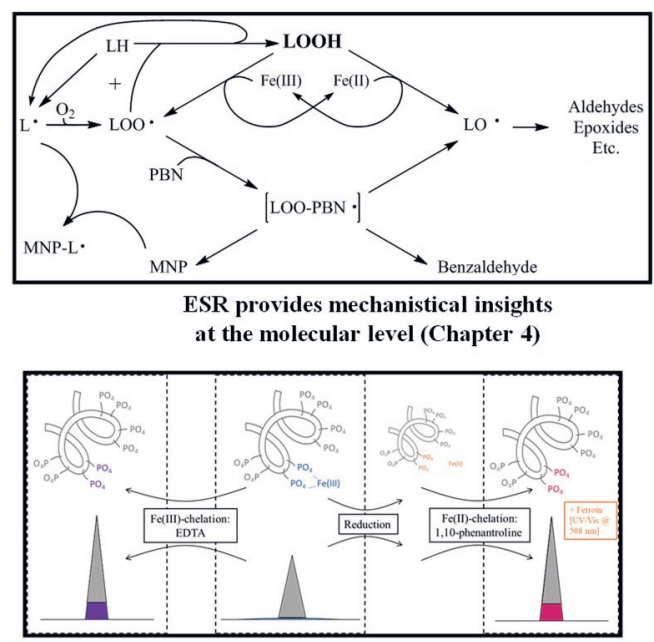

${ }^{31} \mathrm{P}$ NMR provides mechanistical insights at the colloidal level (Chapter 6)

Figure 7.1. Graphical abstract of this thesis outlining the main conclusions. 


\subsection{Strengths and limitations of the developed magnetic resonance methods}

The NMR methods described in Chapters 2, its Appendix and Chapter 3 are, at the time of writing, becoming increasingly popular with fellow researchers in the lipid oxidation field. Compared to chromatographical methods, NMR methods present the advantage to be nondestructive, to allow accurate quantification without standards and to have a high machineto-machine reproducibility. The developed NMR methods allow quantification of three major oxidation classes in one single sample: hydroperoxides and aldehydes can be measured with the ${ }^{1} \mathrm{H}$ NMR (Chapter 2), whereas epoxides can be analysed in the same sample, using ${ }^{1} \mathrm{H}$ ${ }^{13}$ C HSQC NMR (Chapter 3). All three oxidation products include subclasses that were identified and characterised as well (more information in section 7.2.1). If an analyst does not fully elucidate a spectrum, it can, like chromatographic methods, also be used in untargeted multivariate analyses. Importantly, in the journey of unlocking the spectroscopic (NMR) method's potential, we always considered that the methods should also be usable by non-NMR-experts. This included keeping the sample preparation as simple and using as least material $(50-150 \mu \mathrm{L}$ oil) and deuterated solvent $(400-500 \mu \mathrm{L})$ as possible, also reducing chemical waste. The spectral acquisition was fully automated, with a semi-automated data processing and calculations (Appendix-Chapter 2). This means that only basic knowledge on analytics already enables quantification on lipid oxidation product class, where more advanced knowledge will allow one to extract more from the data (such as sub-classes).

On the other hand, the NMR equipment requires high initial investment, which intrinsically limits its widespread availability. In recent years, high-end NMR equipment has become more affordable. Moreover, the low operational costs and the possibility to run samples almost 24/7, result in low cost per sample and greatly shorten the time-to-result, which does make it particularly interesting for companies to use NMR. Another drawback of the NMR methods is lack of specificity at later stages of the oxidation, since here, the large variation of possible oxidation products is present and will likely overlap. Chromatographical methods excel at this stage, as they allow of individual oxidation species. LC-MS is particularly useful for molecular identification of oxidation products that are still attached to the TG-backbone, referred to as non-volatiles (Grüneis et al., 2019; Hollebrands, Varvaki, Kaal, \& Janssen, 2018). Recently, a two-dimensional liquid chromatography (LCXLC) method with evaporative light-scattering detection (ELSD) was developed that also allowed isolation of oxidation products on the basis of the backbone (mono/di/tri-glyceride) (Lazaridi, Janssen, Vincken, Pirok, \& Hennebelle, 2021). Similarly, GC-MS allows characterization of the small secondary oxidation products such as aldehydes, ketones and alcohols (Alberdi-Cedeño, Ibargoitia, \& Guillén, 2020; Beltrán, Ramos, Grané, Martín, \& Garrigós, 2011). On its own, 
NMR is a very versatile tool to obtain a broad comprehension of lipid oxidation. For the full mapping of lipid oxidation pathways, however, complementing the NMR methods with chromatographic methods is essential.

\subsubsection{NMR and ESR for mechanistic understanding of oxidation kinetics}

In this thesis, the term 'mechanistical marker' was used to describe markers that shed a light on any part of the oxidation mechanisms, albeit with different levels of detail. First, one could distinguish based on primary functional group differences of stable (e.g., hydroperoxides, aldehydes, epoxides, unoxidised) or unstable (e.g., alkyl/alkoxyl/peroxyl radicals) oxidation products. Secondly, mechanism-based markers can be characterised based on parent fatty acid (e.g., linoleic acid, oleic acid) or favourable isomers (e.g., cis/trans or cyclization). Thirdly, the furthest level of mechanistical detail would be to fully identify the whole molecule, including position and isomer of each oxidation moiety and full triglyceride composition. In this thesis, this third aspect of mechanistic detail was not explored due to the limitation of magnetic resonance techniques (NMR and ESR) in general. With ${ }^{1} \mathrm{H}$ NMR, one looks at the resonance of proton, which is influenced by its direct chemical environment. Theoretically, with NMR, it is possible to elucidate complex biological structures in pure systems (Kumar, Ernst, \& Wüthrich, 1980). However, in high-throughput lipid oxidation research, there are simply too many different molecules in the mixture that would allow this sophisticated full assignment of the individual products. The first two aspects of mechanistical detail, on the other hand, were thoroughly explored in this thesis.

In Chapters 2 \& 3, we identified the most abundant hydroperoxides, aldehydes and epoxides in mayonnaise with $1 \mathrm{D}{ }^{1} \mathrm{H}$ NMR and 2D HSQC ${ }^{1} \mathrm{H}-{ }^{13} \mathrm{C}$ NMR. Identification of different type of oxidization products within one oxidation class has several benefits. First, knowing with confidence which molecules are measured decreases the chance that unwanted overlapping signals are wrongfully included in the compositional analysis. Unidentified peaks could potentially yield overestimation of the actual amount of oxidation product and reduce the accuracy of the method. Removing such ambiguity from analytical interpretation will result in more profound mechanistical insights. Commonly, trends of individual oxidation classes are investigated (Schröder, Sprakel, Boerkamp, Schroën, \& Berton-Carabin, 2019; Tian et al., 2021). Here, the simplicity and ease of the NMR methods becomes a major advantage, as most of the oxidation products can be captured in one-go by the analyst. With NMR, one looks at functional groups rather than individual molecules, which is an extra advantage since this allows for a correct single molecule assignment of different oxidation classes. Second, monitoring of individual specific markers can help elucidate oxidative mechanisms. This was utilised in Chapter 2, where we confirmed that under higher storage temperatures, the thermodynamically favourable trans-isomers were more dominant in the product 
stoichiometry than the cis-isomers. Besides the isomerisation, the method could also distinguish the parent fatty acid from each hydroperoxide. This allowed us to confirm that linoleic and alpha-linolenic acid were the dominant substrates for lipid oxidation in our tested mayonnaise systems. These individual markers can be readily used as input for multi-variate analysis techniques, such as Principal Component Analysis (PCA), to allow for unsupervised distinction of oxidation pathways. This can be done purely on the NMR results, as exemplified in Chapter 2. It can also be linked with other analytical input, such as GC or sensorial data (Petersen, Kleeberg, Jahreis, Busch-Stockfisch, \& Fritsche, 2012) or with $a$ priori knowledge in Partial Least-Squares regression (PLS) (Cozzolino, Murray, Chree, \& Scaife, 2005; Jacobsen et al., 2000; Weljie, Dowlatabadi, Miller, Vogel, \& Jirik, 2007).

A critical mechanistical marker that is thus far undiscussed are the radicals, which can be assessed with ESR. Since radicals of interest were short-lived, PBN (a spin-trap) was required for their assessment. This spin-trap approach inherently interferes with the free radical processes that are of interest. However, when the reaction mechanisms of the spin-traps are elucidated, opportunities will arise to investigate (parts of the) lipid oxidation mechanisms. In Chapter 4, we showed that the dominating mechanism of PBN in lipid oxidation studies is a reaction where first, the PBN reacts with a peroxy-radical to form an unstable product that generates MNP, benzaldehyde and an alkoxy radical. The MNP also acts as a spin-trap, reacting with a lipid radical to form the stable adduct MNP-L. During the first step, the PBN disallowed any hydroperoxides being formed and pushed the formation of alkoxyl radicals instead. This chapter provided valuable insight in the role of molecules that can modulate oxidation such as PBN and showed the importance of monitoring as many mechanistical markers for oxidation as possible. If only the hydroperoxides were considered, PBN would be classified as 'anti-oxidant', which would not tell the real story. These learnings should not only be applied to similar ESR-related experiments, but more importantly to other anti- and pro-oxidant investigations.

In Chapter 6, ${ }^{31} \mathrm{P}$ NMR was used to investigate the interactions that catalyse oxidation. Even though phosphorous $\left({ }^{31} \mathrm{P}\right) \mathrm{NMR}$ is less sensitive than ${ }^{1} \mathrm{H} \mathrm{NMR}$, it still allowed for assessment of phosvitin, as this is abundantly present in egg yolk. We confirmed that a decreased ironloading on the phosvitin in the water layer of mayonnaise resulted in lower oxidation products in an ASLT. This implied that the phosvitin-Fe is present at the interface, limiting the oxidative stability of the emulsion. In these experiments, the iron-loading of phosvitin was analysed in the aqueous layer of mayonnaise rather than in intact mayonnaises. One way to measure directly in the intact mayonnaise would be via semi-solid-state NMR, using Magic Angle Spinning. This approach was tried, but we were not able to obtain quantitative results due to significant overlap with the phospholipids that were also present, in combination with the low magnetic field $(9.4 \mathrm{~T})$ that was used. Potentially, these challenges can be overcome 
with more sophisticated equipment, such as higher magnetic field strengths, in the future. The method in Chapter 6 was specifically designed and developed to investigate the phosvitin-Fe complexation. The principles and methodologies can be readily expanded into other mechanistical studies. Paramagnetic quenching principles could be utilised in other Febinding studies, in both ${ }^{31} \mathrm{P}$ and ${ }^{1} \mathrm{H}$ NMR. Furthermore, the ${ }^{31} \mathrm{P}$ NMR-method could also be utilised to assess chemical modulation of the phosvitin or other phosphoproteins at the interface during oxidation.

\subsubsection{Exploiting quantitative nature of magnetic resonance methods for predictive modelling}

The methods described in section 7.2.1 were not only providing mechanistical markers: they were also designed to yield absolute quantification results. In Chapters $2 \& 3$, we accurately quantified three of the major oxidation products that are formed in auto-oxidation of food emulsion and oils. One of them being the early oxidation product, the hydroperoxides and the other two the later (secondary) oxidation products: the aldehydes and epoxides. Like traditional methods, the quantification has been done primarily in the lipid layer or a dried organic fraction. This may lead to underestimation of small molecules with a polar functional group, such as late oxidation products like aldehydes, epoxides, ketones and alcohols. On the contrary, if these functionalities are formed on the fatty acid part that is still attached to the TG-backbone, they are more lipophilic, will be detected and can therefore be used in mechanistical studies. The early hydroperoxides are considered too large and lipophilic to readily partition out of the lipid or organic layers, making them a good quantitative mechanistical marker. Since the hydroperoxides were being generated in significant amounts earlier than epoxides, we further investigated whether the former could be used to shorten traditional shelf-life tests. The speed and convenience of the ${ }^{1} \mathrm{H}$ NMR method allowed us to capture a large quantity of hydroperoxide kinetic profiles (>200) of shelf-life tests at $50{ }^{\circ} \mathrm{C}$ to build a prediction model for the ranking of aldehyde on-sets, which is a common way of assessing oxidative stability (Chapter 5). The prediction model that allowed us to make the former statement was built up using mayonnaises with different formulations to introduce robustness in the model. In ASLTs on mayonnaises, hydroperoxide assessment can confidently replace aldehydes for the assessment of oxidative stability, shortening the potential development time from more than a month to less than week. The fact that we built the model using mechanistical markers and a semi-empirical approach allows us to both understand what is happening in the system, and if necessary, to modify the model with future insights. 


\subsection{Reflection on the mechanisms of accelerated shelf-life testing in mayonnaise oxidative stability studies}

\subsubsection{Accelerated shelf-life tests in relation to the consumer}

A point of concern is to which extend conditions of ASLTs are consumer-relevant. The term 'consumer-relevant' is not sharply defined and can refer to the conditions at a consumer's home, store or even transit. In any of these conditions, the storage conditions are mild. The 'ASLTs' are equally poorly defined, as they span the whole range from slightly to heavily accelerated storage conditions. ASLTs can be performed in different ways, by varying oxygen, adding pro-oxidants or as employed in this thesis, by increasing the storage temperature. The latter is what we will focus this discussion on, as it is most employed in both academia and industry. Even then, setting up ASLTs is almost never done in a uniform manner by different research groups. Temperatures can differ significantly, from $40{ }^{\circ} \mathrm{C}$ (Olmedo, Ribotta, \& Grosso, 2019) to $60^{\circ} \mathrm{C}$ (Lehtonen et al., 2016) in emulsions and to even $100{ }^{\circ} \mathrm{C}$ and $150{ }^{\circ} \mathrm{C}$ in vegetable oils (Roman, Heyd, Broyart, Castillo, \& Maillard, 2013). Different storage containers can be used (e.g., headspace vials, Petri dishes or jars) resulting in different matrix-air-surface areas that can influence oxidation rates. Experiments can be done in open or closed systems, and if it is the latter, as in our experiments, it is also important to consider the matrix-to-headspace ratio. All these aspects make comparing analytical results on different ASLTs in literature difficult.

However, consistently monitoring as many oxidation products as possible, as with the provided methods in this thesis, provides a broad overview of different oxidation pathways under chosen ASLT conditions. The use of mechanistical markers allows for a much clearer picture on how the different storage conditions influence the oxidative pathways. For example, we have shown in Chapter 2 that increasing the storage temperature favours the generation of trans-isomers rather than the cis-isomers, and at higher temperatures, both aldehyde and epoxide generation followed hydroperoxides much quicker than at lower temperatures (Chapters 2 \& 3). At room temperature or chilled storage conditions, using hydroperoxide quantification and the prediction model in Chapter 5 will not generate oxidative ranking in less than a week, simply because the generation of hydroperoxides under mild storage conditions take considerably longer (Chapter 2). To explore early oxidation markers under non-accelerated conditions, ESR was used (Chapter 4). We indeed found that spin adduct generation over time was indicative for the oxidative stability of the emulsion at room temperature conditions (within a week). A limitation of this study, however, was that it was only tested on a series with a varying quantity of a secondary antioxidant, the chelator EDTA. It needs to be investigated that when using primary antioxidants (i.e., radical 
scavengers), which directly react with the radicals and thus compete with the PBN and MNP, proper oxidative ranking would also be achievable. Nonetheless, if the mode-of-action of both the anti-oxidant and its interaction with the spin-trap is mapped, then ESR can be used to assess oxidative ranking under consumer-relevant conditions.

Another important point of discussion when translating from ASLT to consumer relevant conditions is the role that oxygen transport and availability play in commercial packages, such as jars. This could result into a heterogenous distribution of oxidation products, where one can expect more oxidation close to the top of the jar, with the bottom still relatively fresh. In the same line of thought, increasing surface diameter will allow more oxygen to diffuse into the matrix, impacting early oxidation kinetics. Having a quick and accurate tool as provided in Chapter 2 allows for spatial sampling, which is necessary for mapping the influence of oxygen transport and distribution in shelf-life testing. Ultimately, it can feed into mechanistic models (see section 7.4.4).

\subsubsection{The pivotal role of hydroperoxides}

Traditionally, lipid oxidation chemistry describes the initiation phase with spontaneous radical abstraction from an unsaturated lipid, where the resulting alkyl radicals react with oxygen to form peroxyl radicals. These peroxyl radicals then propagate to hydroperoxides and new free alkyl radicals, continuing until the free radical is quenched. This means that when acquiring processed oil from a supplier, the chances are high that there will already be traces of hydroperoxides present. Indeed, in this thesis, all oxidative studies started with oils that were considered fresh, but did have minor hydroperoxide traces $(<1 \mathrm{mmol} / \mathrm{kg})$. Since the main product of our studies, mayonnaise, consisted of iron-loaded egg yolk, we focused on the role of iron on oxidative mechanisms. In Chapters 2 \& 3, we showed that aldehydes and epoxides show similar kinetic behaviour, suggesting that both compound classes had the same precursor. By utilizing ESR in combination with PBN spin-trapping, we confirmed that the catalytic formation of alkoxyl radicals from hydroperoxides is the main driver for secondary oxidation in these systems (Chapter 4). Here, we observed that when LOOHs were exposed to the iron redox cycle this yielded LO and LOO radicals, that especially in the presence of PBN resulted in extremely high secondary oxidation with a low amount of stable hydroperoxides. Where normally the LOO react with an $\mathrm{LH}$, they were now trapped with the PBN, readily forming even more LO radicals. This showed that although LOOHs are the intermediates in the formation of LO radicals, that it is essential that they are being kept stable since this will hamper secondary oxidation product formation. It also meant that when the LOO- is captured by another scavenger (in this case the PBN), that this does not directly imply that the oxidation is being effectively slowed down. Moreover, hydroperoxide 
kinetics under ASLT conditions have a secondary acceleration phase which was concentration dependant. This concentration was interpreted as the Critical Micellar Concentration (CMC), as hydroperoxides have a lipophilic tail and a hydrophilic head that could (in combination with other amphiphilic molecules) could form (inverse) micelles. Here, under the same storage conditions, the time it took for the hydroperoxides to reach this CMC correlated to the onset of secondary oxidation (aldehydes). The combination of these chemical and physical aspects makes the LOOHs play a pivotal role in lipid oxidation in mayonnaise. In this matrix, we indeed showed that chelating the iron with EDTA (Chapter 6) reduced the chance of iron to enter the oxidative track and limited further propagation (Figure 7.2). In mayonnaise systems, this means that utilizing molecules with chelator-like properties should be preferred over those with primarily radical scavenging capacity due to the reaction of LOOHs with transitional metals. This does not only hold for mayonnaise, where indeed transition metals are present in excessive amounts, but also in other matrices. In recent years, studies in other matrices describe the same necessity of considering LOOH as a crucial piece of the puzzle (Laguerre, Tenon, Bily, \& Birtić, 2020; Schaich, Xie, \& Bogusz, 2017). 


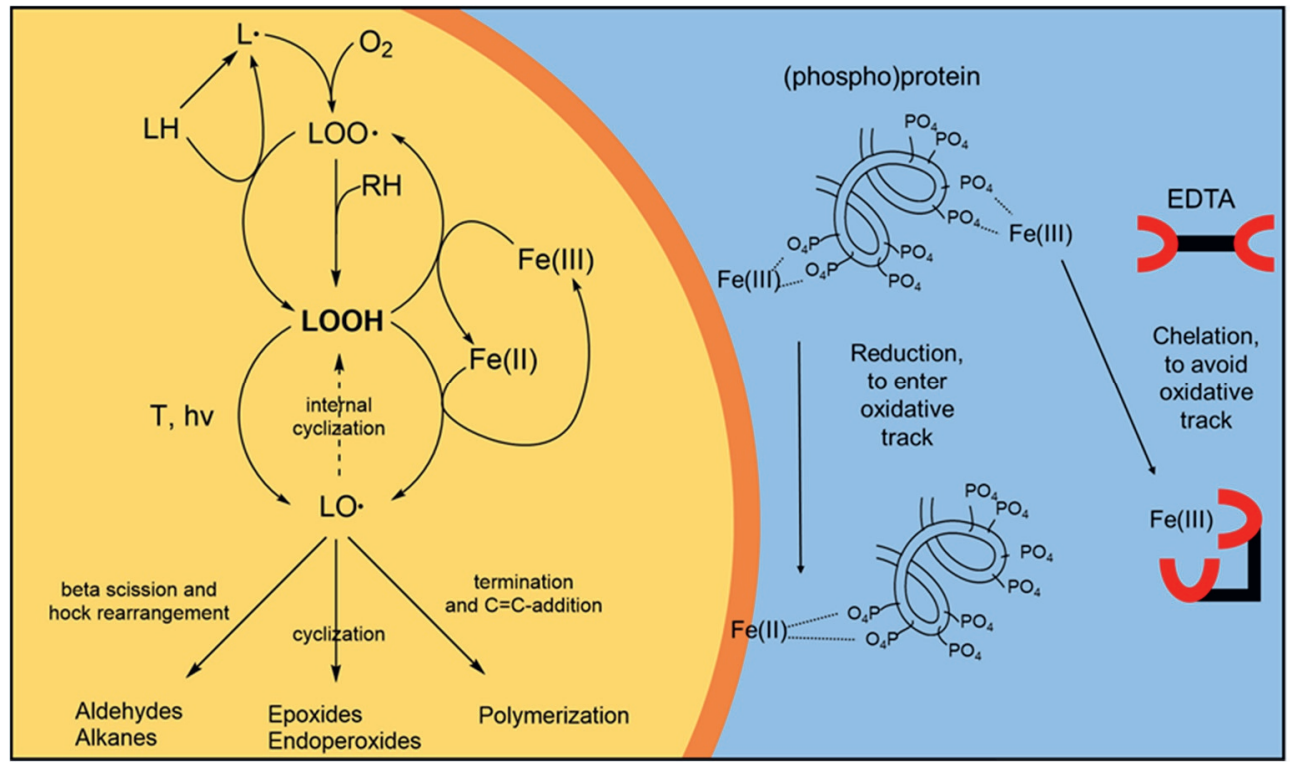

Figure 7.2. Schematic description of the lipid oxidation pathways in oil (left), emphasizing the central role of the hydroperoxides $(\mathrm{LOOH})$ and the catalytic function of iron, a transition metal. On the right, the interplay between iron and a phosphoprotein and a chelator (EDTA) is schematically depicted. Here, unchelated iron is able to enter the oxidative track, whereas chelated iron stays mainly in the water layer, away from the oxidative interface.

\subsection{Perspectives}

The scope of this thesis laid on mechanism-based early markers for lipid oxidation in mayonnaise, which included mechanistic underpinning of these markers to discriminate the involved dominant pathways (Figure 7.3). In this section, I would like to offer some perspectives on how these analytical methods and pathways can be translated to other matrices or oil, and what further developments in the current methods can be still achieved. Thoughts on the requirement of sensorial analyses will be discussed. Lastly, colloidal aspects of lipid oxidation in mayonnaise are reflected upon, a topic that was touched upon in Chapter 5 and Chapter 6. Here, I share some thoughts on what the future might hold with regards to further elucidation of colloidal mechanisms. 


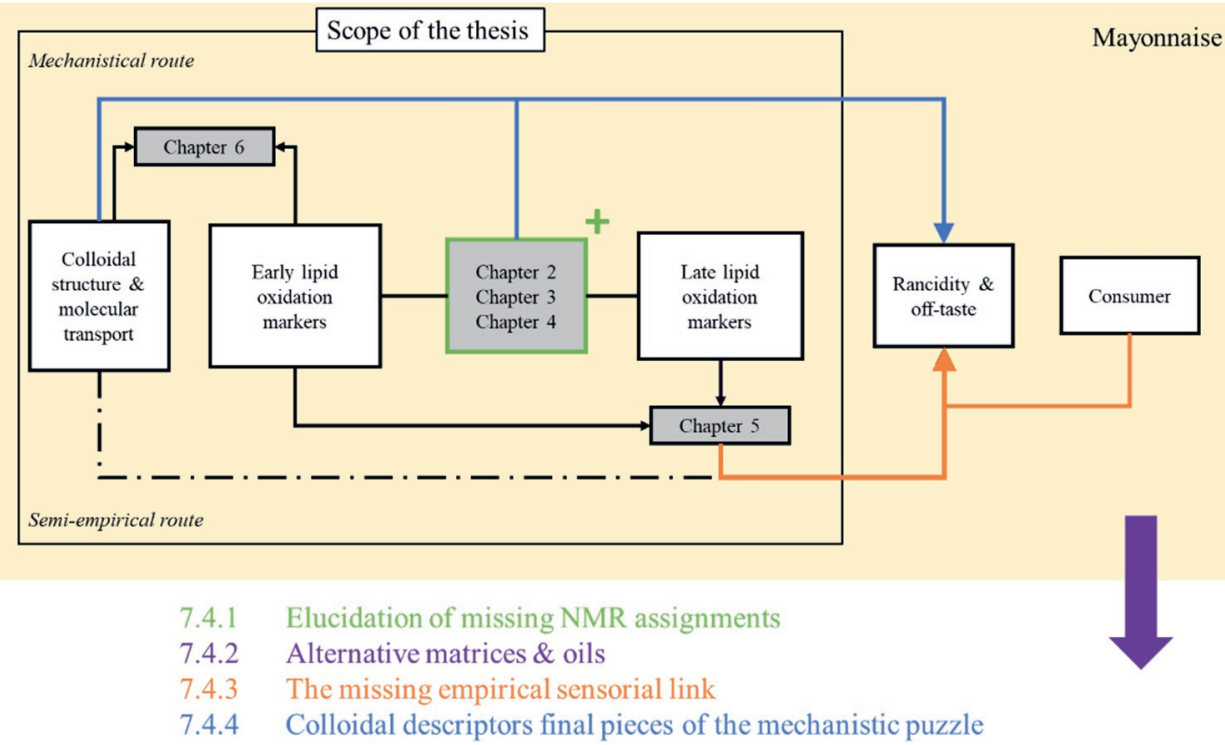

Figure 7.3. Schematic overview of the perspectives sections with how each section can be related to the scope of this thesis.

\subsubsection{Elucidation of missing NMR assignments}

As briefly touched upon before, not every peak in the NMR spectra (both 1D and 2D) was identified (Chapters $2 \& 3$ ). Peaks can be identified chemically, by using pure compounds or isolated standards, or spectroscopically, by using multidimensional NMR experiments such as NOESY, TOCSY and HSQC. As in Chapter 2, where we used a combination of both approaches to assign the majority of the hydroperoxides based on parent free fatty acid and stereoisomerism (Figure 7.4). There is further opportunity to expand this LOOH assignment in a similar way to include the more unsaturated fatty acid classes as well, e.g., EPA and DHA. Recently, a new method has been published where LOOHs are identified in even more detail, using computational studies, albeit on model compounds (Ahmed, Siddiqui, Choudhary, \& Gerothanassis, 2019). Direct application of this method on real food samples would be very difficult as such due to the high number of overlapping signals. This approach does potentially allow the identification of other late oxidation products such as ketones and alcohols. These compounds were not identified in this thesis since the amounts of these products are expected to be low, where the focus lay on the major mechanistical markers. 

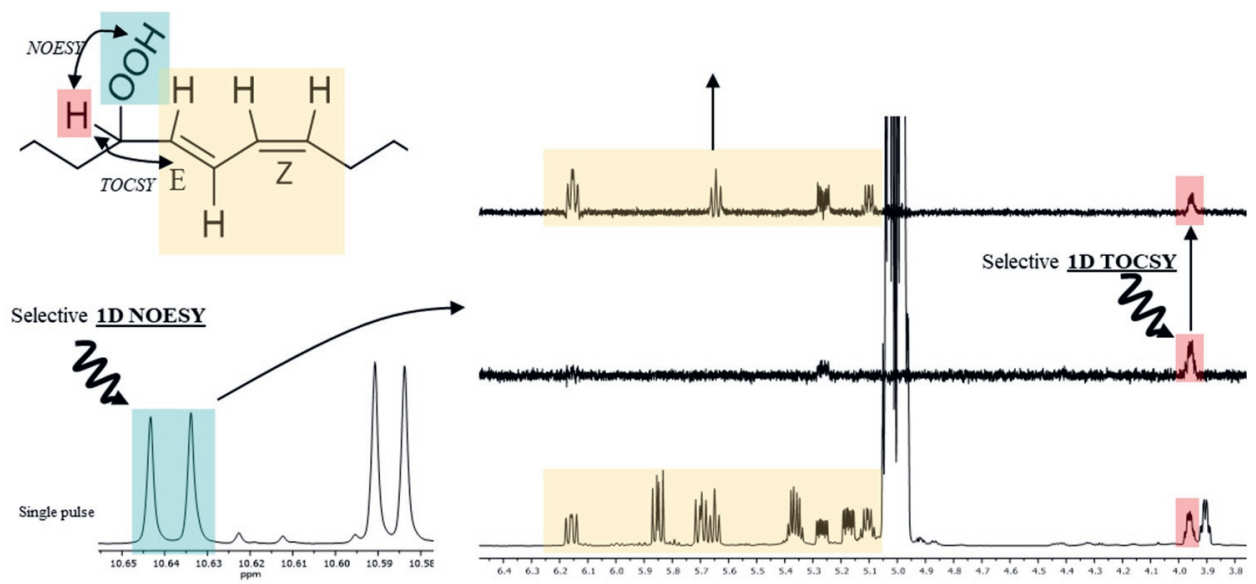

Figure 7.4. Detailed example on the elucidation of one specific isomer of the LA-hydroperoxides. A combination of a model compound and two-dimensional spectroscopic principles were used to elucidate the final structure (in this example the cis,trans-LA-isomer).

Furthermore, when oxidation is followed into late stages of oxidation products, a wide variety of unidentified aldehydes and epoxides were formed (Chapters $2 \& 3$ ). In this thesis, the identification of unidentified peaks was not pursued to the same extent as for the hydroperoxides. Further elucidation of these peaks still has a significant amount of unlocked potential to contribute to further mechanistical understanding of lipid oxidation. As a concrete example, we show the kinetics of four elucidated abundant epoxides in Chapter 3 (Figure 7.5). The kinetics of these epoxides in simple storage trial $\left(60^{\circ} \mathrm{C}\right.$, RP oil, unlimited $\mathrm{O}_{2}$ ) is distinct: the cis- and trans-epoxides follow a different trajectory than the epoxyhydroperoxides. This could be explained due to relative instability of the hydroperoxide moieties, generating more alkoxyl radicals in the process. This shows the potential of elucidating even more oxidation products regarding the development of specific mechanismbased oxidation markers. 


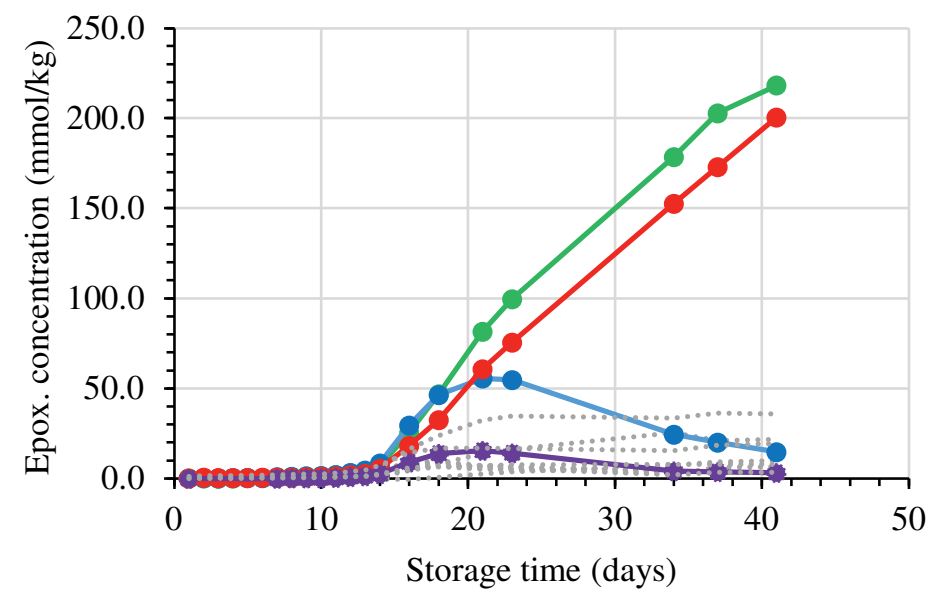

Figure 7.5. Generation of epoxides as a function of storage time. Different colours represent different epoxides in correspondence to Figure 3.3: Trans-Epox (red), Cis-Epox (green), Trans-Epox-OOH (blue), Cis-Epox-OOH (purple), unidentified epoxides (grey)

\subsubsection{Alternative matrices \& oils}

In this section, the expansion of the provided methods to other oil types and emulsions is explored. The fatty acid composition of edible oils such as soybean and sunflower oil are very comparable to that of rapeseed oil, and are expected to yield similar results. When expanding the work to oils that are substantially lower in unsaturation, such as olive oil, observed mechanisms might very well differ or methods need re-evaluation to accommodate for a lower amount of oxidation products under the same storage conditions. On the other end of spectrum lie the fish oils and other oil with a large amount of highly unsaturated fatty acids. Where, in edible oils such as rapeseed oil, the amount of DHA and EPA is negligible, in fish oil, these are present in significant amounts. Thus, where the spectral assignment in Chapter 2 is limited to oleic, linoleic and linolenic acid hydroperoxides, there will be a need to expand this for larger and more unsaturated fatty acids. This also applies to the aldehydes and epoxides that can be formed and thus would require extra assignments.

Besides the type of oil, the way the oil is incorporated in the matrix also plays a significant role. In high-fat oil-in-water emulsions such as mayonnaise, the oil layer can be rapidly and representatively extracted from the emulsion by a simple freeze-thaw cycle. This is partly due to the surfactants in the egg yolk that are incapable to maintain the stability of the oil droplets during the thawing process. In other type of O/W emulsions, high- and low-fat, stabilised by other surfactants/proteins or by increasing the viscosity of the water layer, this 
freeze-thaw cycle is often not sufficient to extract a representative oil layer. Here, one needs to perform a chemical extraction, such as Bligh-Dyer or hexane:isopropanol, to obtain a clean oil layer. Similarly, extraction steps are often required when working with water-in-oil or spray-dried emulsions. On the one hand, the methods that are described in this thesis require some more work when having to include an extraction step. On the other hand, the qNMR methods do allow these extractions to be performed on a rather small scale, significantly reducing the amount of work and chemicals that are required in these steps.

In Chapter 5, we developed a prediction model for the on-set of aldehydes in mayonnaises, based on the hydroperoxide formation. The set of chosen environmental settings limit the direct translation of this prediction model to other matrices or storage conditions. This, however, does not mean that the learnings described in Chapter $\mathbf{5}$ do not apply to other systems of interest. For example, changing the temperature or the headspace-to-emulsion ratio will likely have an impact on the $\mathrm{CC}_{\mathrm{LOOH}}$ or the slope of the prediction model, which would only require a straightforward recalibration of the model.

\subsubsection{The missing sensorial link}

In lipid food products, the main driver for investigating lipid oxidation is to prevent offflavour generation during storage. Off-flavour profiles in these products will vary depending on oil type, matrix type, extra ingredients, and environmental conditions. For example, products using flavourings or herbs might mask off-flavour generation, while the use of fish oil instead of a vegetable oil will generate a totally different overall rancidity profile due to different oxidation products being formed. Preferably, these off-flavour profiles can be predicted with early mechanistical markers to reduce product development time. The correlation between generic early markers and sensory profile has been investigated (Jacobsen, 1999). Here, the authors found that PV and AV correlated poorly with an overall flavour score, but volatiles, as provided with their GC analysis, did correlate well to offflavour. Indeed, these volatile aldehydes are known as the major contributors of off-flavour and perceived rancidity (Kochhar, 1996). We demonstrated in Chapter 5, that, by using early hydroperoxide compound class kinetics, we can accurately predict the (non-volatile) aldehyde on-set under accelerated shelf-life conditions. By extension, this approach could potentially yield similar prediction models between early mechanistical markers and the volatiles. This would require modelling at sub-class level (e.g., hexanal, 9-trans,cis-LALOOH), instead of the currently used compound class level (e.g., aldehydes, hydroperoxides). For the early markers, these different compound sub-classes can be quantified by NMR, where late oxidation products can be quantified by advanced HS-GCMS methods. Besides this prediction model to link early and late mechanistical markers, the correlation between these late markers and off-flavour should be established using sensory 
panels. This combination would allow the use of early mechanistical markers as direct predictors of the sensory profile.

\subsubsection{Colloidal descriptors: final pieces of the mechanistic puzzle?}

To fully elucidate lipid oxidation in food emulsion using mechanistical modelling, the whole system should be described accurately using model descriptors (Figure 7.6). These model descriptors include the environmental descriptors, which are partly controlled or easy to monitor. They also include the chemical descriptors, i.e., the oxidation products at a molecular of moiety level, which was the focus of the first chapters. To finalise this mechanistical puzzle colloidal descriptors are required, which capture the transport, interface and/or micellar interactions in the emulsions. Examples of such colloidal descriptors can be found in Chapter 6, where we tried to link the phosvitin-Fe binding at the interface to early oxidation and indirectly in Chapter 5, where the secondary acceleration phase in the $\mathrm{LOOH}$ curve was linked to the formation of inverse micelles. These analyses were done on samples that underwent accelerated shelf-life tests, in which was assumed that the intra-droplet kinetics are more important than inter-droplet kinetics. This assumption was made on basis of a theoretical framework (Budilarto \& Kamal-Eldin, 2015; Chaiyasit, Elias, McClements, \& Decker, 2007).

Current methodological approaches do not cover the full range of colloidal descriptors that are required to test the several hypotheses on the behaviour of lipid oxidation products in emulsions/colloidal systems as discussed in Chapter 1. To fully understand colloidal interactions, we would need to monitor lipid oxidation in the relevant places in an undisturbed system and follow it over time. Such a spatiotemporal approach would have the potential to yield inter- and intra-droplet dynamics learnings. In recent years, there have been significant developments in this area, notably by means of Confocal Laser Scanning Microscopy (CLSM) and flow cytometry. With CLSM, colleagues were able to assess lipid and protein oxidation in mayonnaises at three different places in a stable emulsion using BODIPY 665/676 fluorescence emission and protein auto-fluorescence (Yang, Verhoeff, Merkx, van Duynhoven, \& Hohlbein, 2020). Individual droplets could be tracked as a function over time, allowing for the resolving of kinetics on a unique level of detail. In this study, this novel technique demonstrated that ascorbic acid has different roles on both lipid and protein oxidation on the colloidal interface. Ascorbic acid was always pro-oxidant on the protein oxidation and depending on the presence or absence of lipid-soluble antioxidants (such as tocopherol), it behaved respectively anti- or pro-oxidative. This shows that this novel CSLMtechnique can be deployed to spatially resolve lipid oxidation in colloidal systems, potentially generating insights in the transport mechanisms at play. In parallel, another research group explored the potential of using flow cytometry to resolve mass transfer kinetics of lipid 
oxidation products in model oil-in-water emulsions (Li, McClements, \& Decker, 2020). Flow cytometry is a technique that can monitor individual oil droplets, but contrary to CLSM, this cannot be done in situ. In these model emulsions, TAG-oxidation products were not able to migrate between droplets without the presence of surfactants. Free fatty acids, and other small oxidation products, such as 2,4-decadienal, were able to travel through the aqueous layer at surfactant concentrations below their CMC. The authors hypothesised that the pro-oxidative role of salt in these systems could be linked to further lowering the CMC of present surfactants, further improving mass transport of oxidation products to un-oxidised droplets. Flow cytometry enables future endeavours to further elucidate these mass transport phenomena. It is important to consider, however, that both spatiotemporal techniques require a fluorescent dye that might modulate the actual mechanism, as with the PBN spin-trap (Chapter 4).

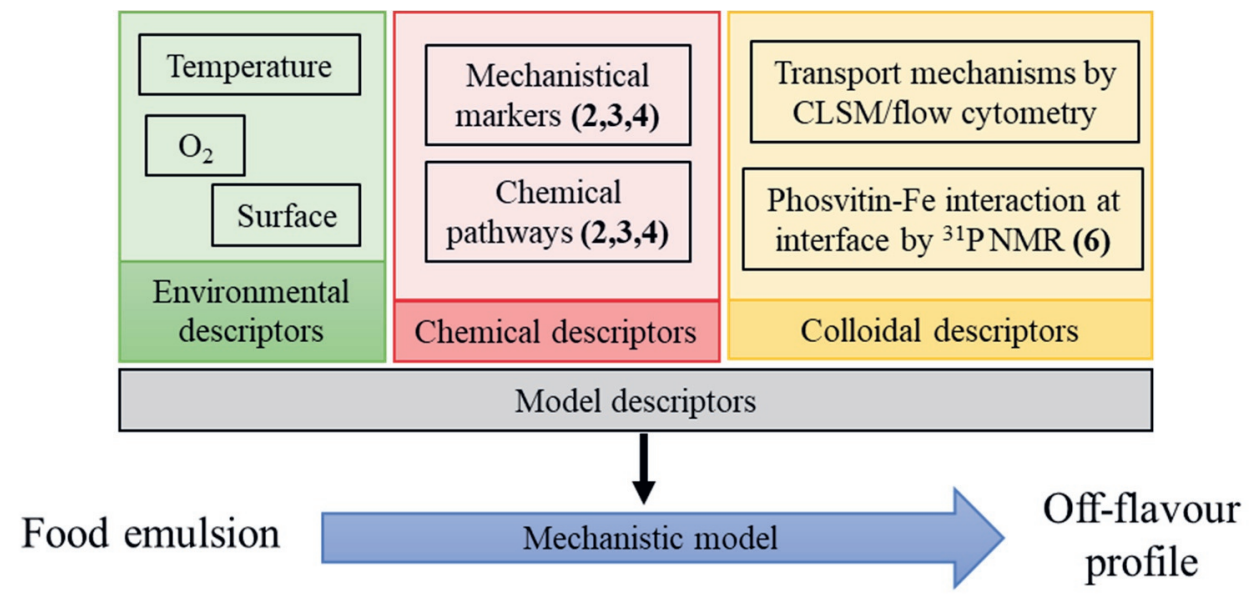

Figure 7.6. Schematic overview of the interplay required for full mechanistic modelling. The numbers between brackets indicate the chapter in this thesis that correspond to that descriptor.

The further development of these highlighted spatiotemporal techniques will provide the necessary tools that, in combination with chemically resolved methods, will allow for further elucidation of these complex colloidal mechanisms in food emulsions. 


\subsection{Literature}

Ahmed, R., Siddiqui, H., Choudhary, M. I., \& Gerothanassis, I. P. (2019). 1H-13C HMBC NMR experiments as a structural and analytical tool for the characterization of elusive trans/cis hydroperoxide isomers from oxidized unsaturated fatty acids in solution. Magnetic Resonance in Chemistry, 57(4), S69-S74.

Alberdi-Cedeño, J., Ibargoitia, M. L., \& Guillén, M. D. (2020). Effect of the enrichment of corn oil with alpha-or gamma-tocopherol on its in vitro digestion studied by $1 \mathrm{H}$ NMR and SPME-GC/MS; formation of hydroperoxy-, hydroxy-, keto-dienes and keto-E-epoxy-E-monoenes in the more alpha-tocopherol enriched samples. Antioxidants, 9(3).

Beltrán, A., Ramos, M., Grané, N., Martín, M. L., \& Garrigós, M. C. (2011). Monitoring the oxidation of almond oils by HS-SPME-GC-MS and ATR-FTIR: Application of volatile compounds determination to cultivar authenticity. Food Chemistry, 126(2), 603-609.

Budilarto, E. S., \& Kamal-Eldin, A. (2015). The supramolecular chemistry of lipid oxidation and antioxidation in bulk oils. Eur J Lipid Sci Technol, 117(8), 1095-1137.

Chaiyasit, W., Elias, R. J., McClements, D. J., \& Decker, E. A. (2007). Role of physical structures in bulk oils on lipid oxidation. Critical Reviews in Food Science and Nutrition, 47(3), 299-317.

Cozzolino, D., Murray, I., Chree, A., \& Scaife, J. R. (2005). Multivariate determination of free fatty acids and moisture in fish oils by partial least-squares regression and nearinfrared spectroscopy. LWT - Food Science and Technology, 38(8), 821-828.

Grüneis, V., Fruehwirth, S., Zehl, M., Ortner, J., Schamann, A., König, J., \& Pignitter, M. (2019). Simultaneous Analysis of Epoxidized and Hydroperoxidized Triacylglycerols in Canola Oil and Margarine by LC-MS. Journal of Agricultural and Food Chemistry, 67(36), 10174-10184.

Hollebrands, B., Varvaki, E., Kaal, S., \& Janssen, H.-G. (2018). Selective labeling for the identification and semi-quantification of lipid aldehydes in food products. Analytical and Bioanalytical Chemistry, 410(22), 5421-5429.

Jacobsen, C. (1999). Sensory impact of lipid oxidation in complex food systems. Lipid / Fett, 101(12), 484-492.

Jacobsen, C., Hartvigsen, K., Lund, P., Thomsen, M. K., Skibsted, L. H., Adler-Nissen, J., . . . Meyer, A. S. (2000). Oxidation in fish oil-enriched mayonnaise. 3. Assessment of the influence of the emulsion structure on oxidation by discriminant partial least squares regression analysis. European Food Research and Technology, 211(2), 8698.

Kochhar, S. (1996). Oxidative pathways to the formation of off-flavours.

Kumar, A., Ernst, R. R., \& Wüthrich, K. (1980). A two-dimensional nuclear Overhauser enhancement (2D NOE) experiment for the elucidation of complete proton-proton cross-relaxation networks in biological macromolecules. Biochemical and Biophysical Research Communications, 95(1), 1-6.

Laguerre, M., Tenon, M., Bily, A., \& Birtić, S. (2020). Toward a Spatiotemporal Model of Oxidation in Lipid Dispersions: A Hypothesis-Driven Review. European Journal of Lipid Science and Technology, n/a(n/a), 1900209.

Lazaridi, E., Janssen, H.-G., Vincken, J.-P., Pirok, B., \& Hennebelle, M. (2021). A comprehensive two-dimensional liquid chromatography method for the 
simultaneous separation of lipid species and their oxidation products. Journal of Chromatography A, 1644, 462106.

Lehtonen, M., Teräslahti, S., Xu, C., Yadav, M. P., Lampi, A. M., \& Mikkonen, K. S. (2016). Spruce galactoglucomannans inhibit lipid oxidation in rapeseed oil-in-water emulsions. Food Hydrocolloids, 58, 255-266.

Li, P., McClements, D. J., \& Decker, E. A. (2020). Application of flow cytometry as novel technology in studying lipid oxidation and mass transport phenomena in oil-inwater emulsions. Food Chemistry, 315, 126225.

Olmedo, R., Ribotta, P., \& Grosso, N. R. (2019). Decrease of chemical and volatile oxidation indicators using oregano essential oil combined with BHT in sunflower oil under accelerated storage conditions. Journal of food science and technology.

Petersen, K. D., Kleeberg, K. K., Jahreis, G., Busch-Stockfisch, M., \& Fritsche, J. (2012). Comparison of analytical and sensory lipid oxidation parameters in conventional and high-oleic rapeseed oil. European Journal of Lipid Science and Technology, 114(10), 1193-1203.

Roman, O., Heyd, B., Broyart, B., Castillo, R., \& Maillard, M.-N. (2013). Oxidative reactivity of unsaturated fatty acids from sunflower, high oleic sunflower and rapeseed oils subjected to heat treatment, under controlled conditions. LWT - Food Science and Technology, 52(1), 49-59.

Schaich, K. M., Xie, J., \& Bogusz, B. A. (2017). Thinking outside the classical chain reaction box of lipid oxidation: Evidence for alternate pathways and the importance of epoxides. Lipid Technology, 29(9-10), 91-96.

Schröder, A., Sprakel, J., Boerkamp, W., Schroën, K., \& Berton-Carabin, C. C. (2019). Can we prevent lipid oxidation in emulsions by using fat-based Pickering particles? Food Research International, 120, 352-363.

Tian, L., Kejing, Y., Zhang, S., Yi, J., Zhu, Z., Decker, E. A., \& McClements, D. J. (2021). Impact of tea polyphenols on the stability of oil-in-water emulsions coated by whey proteins. Food Chemistry, 343, 128448.

Weljie, A. M., Dowlatabadi, R., Miller, B. J., Vogel, H. J., \& Jirik, F. R. (2007). An inflammatory arthritis-associated metabolite biomarker pattern revealed by $1 \mathrm{H}$ NMR spectroscopy. Journal of Proteome Research, 6(9), 3456-3464.

Yang, S., Verhoeff, A. A., Merkx, D. W. H., van Duynhoven, J. P. M., \& Hohlbein, J. (2020). Quantitative Spatiotemporal Mapping of Lipid and Protein Oxidation in Mayonnaise. Antioxidants, 9(12), 1278. 

Summary 
Lipid oxidation is a major concern in the food industry as it is one of the main contributors to the limited shelf life of lipid-based food products. This is a particular concern for food emulsions with high amounts of unsaturated fats, such as mayonnaise. In the food industry, accelerated shelf-life tests (ASLTs) are often applied to assess the oxidative stability of different formulations. These ASLTs are not used without drawbacks. First, it is well recognised that shelf-life testing under accelerating conditions may induce different mechanisms and therefore, different stability rankings than when performed under consumerrelevant conditions. Secondly, even under accelerated conditions, it can still take weeks of shelf-life before (late) off-flavour compounds can be detected. Thirdly, it is difficult to infer the involved mechanism from these late lipid oxidation products. To optimise formulation and processing of oxidation-stable food emulsions in a more rapid and rational manner there is a clear need for shelf-life tests that provide early (hours-days) markers that quantitatively predict generation of off-flavour and give an indication of the involved oxidative mechanism(s). The main goal of the thesis is to discover these mechanism-based early markers for lipid oxidation in food emulsions, possibly under consumer relevant conditions. The mechanistic underpinning of these markers should also allow the discrimination of the involved dominant pathways. To achieve this, we intend to develop rapid and quantitative NMR and ESR methods that cover major lipid oxidation initiators and intermediates.

In Chapter 2, we described the development of an NMR method for the quantitative assessment of both hydroperoxides and aldehydes, respectively primary and secondary oxidation products, using band-selective selective ${ }^{1} \mathrm{H}$ NMR pulse excitation. ${ }^{1} \mathrm{H}$ NMR signals of hydroperoxides were assigned in a fatty acid and isomer specific way. The method allowed quantification of hydroperoxides and aldehydes with high throughput and high dynamic range at levels of $0.03 \mathrm{mmol} / \mathrm{kg}$ with high precision $\left(\mathrm{RSD}_{\mathrm{R}}=5.9 \%\right)$. Explorative multivariate data modelling of the quantitative ${ }^{1} \mathrm{H}$ NMR profiles revealed that shelf-life temperature had a significant impact on lipid oxidation mechanisms. In the Appendix of Chapter 2, the execution of the ${ }^{1} \mathrm{H}$ NMR method has been described in a step-by-step manner. Here, we also explained how the ${ }^{1} \mathrm{H}$ NMR method could be applied to different type of vegetable oils and oil-extracts. 
In Chapter 3, NMR was further explored to include the epoxides that were not quantified in Chapter 2. Here, we developed a $2 \mathrm{D}{ }^{1} \mathrm{H}_{-}{ }^{13} \mathrm{C}$ HSQC method that allows for a quantitative assessment of epoxides in real food products. This allowed us to include the major epoxide contribution in the assessment of environmental impact on lipid oxidation pathways.

Different classes of epoxides were distinguished, based on stereospecificity and additional oxidation moieties. We found limited potential for epoxides as a sole early marker. Combined assessment of hydroperoxides and epoxides will allow for capturing of main radical mechanisms.

In Chapter 4, we examined the potential of ESR for early assessment of lipid oxidation. By following spin adducts over time, we could derive the oxidative stability of an emulsion. Importantly, already at mild $\left(=<50{ }^{\circ} \mathrm{C}\right)$ shelf-life conditions, the PBN-OOL adducts rapidly degrades to MNP, benzaldehyde and alkoxy-radicals. In contrast to what is often believed, the observed ESR-signal predominantly originated from the adducts of MNP and alkyl radicals. Furthermore, we showed that the trapping of LOO radicals and subsequent generation of LO radicals impacted downstream reaction pathways, which precludes mechanistic studies in the presence of PBN.

In Chapter 5, we used the early oxidation products to train a prediction model for late oxidation kinetics. To this, we used an extensive set of quantitative hydroperoxide, and aldehyde data obtained in a wide range of emulsion formulations under accelerated shelf-life tests $\left(50{ }^{\circ} \mathrm{C}\right)$. We showed that a sigmoidal Foubert-adapted model could be used to describe the initial phase of hydroperoxide generation. The point at which this function reached the proposed $\mathrm{CC}_{\mathrm{LOOH}}$ of $38 \mathrm{mmol} / \mathrm{kg}$ was used as an accurate predictor for the onset of aldehyde generation in mayonnaise formulations. LOOH measurements within a few days of shelf-life are sufficient to accurately predict the aldehyde onset which takes place at a time scale of weeks. Furthermore, the parameters that describe the Foubert function, i.e., the induction delay (f0) and the growth rate $(\mathrm{K})$, could be used to discriminate primary and secondary antioxidative mechanisms. 
In Chapter 6, a ${ }^{31}$ P NMR based method was developed to quantify loading of phosvitin with $\mathrm{Fe}(\mathrm{III})$ and its reductive release. Both features could be quantified in model phosvitin solutions by exploiting the paramagnetic broadening of ${ }^{31} \mathrm{P}$ NMR signal of phosphoserine residues by $\mathrm{Fe}(\mathrm{III})$. This method was then successfully applied to quantify the phosvitin$\mathrm{Fe}(\mathrm{III})$ loading in mayonnaise water phase by liquid NMR, whereas ${ }^{31} \mathrm{P}$ NMR MAS could only provide a qualitative measure. The ${ }^{31} \mathrm{P}$ NMR method showed that lowering the Fe(III) loading of phosvitin in mayonnaise by EDTA-chelation directly correlated with lipid oxidation stability.

In Chapter 7, the insights and implications from the previous chapters are discussed and put into a broader perspective. First, the strengths and limitations of the presented methods are reflected upon for both spectroscopic and modelling methods. The effect of mechanistical markers on the interpretation of accelerated shelf-life tests and the pivotal role of hydroperoxides in mayonnaise are then discussed, followed by the wider applicability of the provided methods, including other oil types and matrices. Lastly, future perspectives to further elucidate the physico-chemical interplay in emulsions and prediction of sensorial properties are outlined. 


\section{Acknowledgements}


Ik wil bij deze graag alle mensen bedanken die mayonaise eten en het niet kunnen waarderen als deze ranzig smaakt. Zonder jullie had ik nooit zo'n leuk project kunnen doen.

Einde.

Of toch niet.

John, ik wil jou graag als eerste bedanken. Allereerst dat je me in september 2013 als verse scheikunde-master had aangenomen als NMR-analist in je team en veel vertrouwen in me had. Wat tot uiting kwam toen je mij in 2015 subtiel hebt weten te overtuigen om toch ook een $\mathrm{PhD}$ te gaan doen. In 2016 kwam de kans echt om dit traject in te gaan, onder de paraplu van het TNI-project, wat jij had opgetuigd in samenwerking met Food Chemistry. Gedurende de vijf jaar hierna (2016-2021) heb je voor mij gefungeerd als promotor voor mijn PhDtraject en als baas/hoofd van ons NMR-team. In deze periode heb ik enorm veel van je kunnen leren, zowel op academisch en NMR-vlak, als de combinatie met het bedrijfsleven, hiervoor bedankt.

Aansluitend zou ik ook graag het NMR-team willen bedanken. Niels, ik vond het enorm fijn dat jij al die jaren mijn vloeistof-NMR-kompaan was, ik heb enorm veel van je kunnen leren, met name op technisch vlak. Verder waardeer ik het enorm dat je altijd bereid bent om te helpen en beschikbaar was voor een brainstorm. Gert-Jan, Ruud en Adrian, ik wil jullie ook graag bedanken voor de fijne collega's die jullie zijn. Ik heb veel van jullie opgestoken tijdens onze pauzes en wandelingen, zowel over zaken betreft werk en buiten werk om. GertJan, ik kan met jou altijd heerlijk onze gedeelde passie met alles betreft sport en gezondheid delen. Doris, ik heb veel van jou kunnen leren omtrent modeling in combinatie met analytische data, danke schön. Courtney, bedankt voor het overnemen van mijn oude baan: ik zie dat het in goede handen is!

Ewoud, ik kon en kan bij jou altijd terecht voor een goed gesprek, met name hoe je een $\mathrm{PhD}$ moet combineren met een Unilever baan. Ik heb met je altijd leuke inhoudelijke gesprekken kunnen voeren betreft NMR, vet-oxidatie, modelering en de combinatie ervan, waar ik veel van je heb kunnen leren. Maar niet te vergeten, kan ik met jou, Ruud en Marjolein enorm mooi ouwehoeren over onze gezamenlijke passie: F1 en dan vooral Max.

En niet te vergeten: ik zou de microscopisten aan 'het einde van de gang' graag willen bedanken. Lia, Peter (N.), Patricia, Martin, Mazhar, Lonneke, bedankt voor jullie inzichten en gezelligheid!

Boudewijn, Wilma, Herrald, Hans-Gerd, van jullie heb ik veel geleerd over chromatografie, en wat de krachten zijn van deze methodes betreft vet-oxidatie. Hierdoor heb ik me bij de ontwikkeling van de NMR-methodes kunnen richten op het complementaire 
aspect in plaats van het competitieve. Ruud (P.) en Martijn, jullie bedankt voor het uitvoeren van de GC-analyses in hoofdstuk 2. Christiaan, jij hebt mij geleerd hoe je goede mayonaises moet maken, en deze vervolgens op een handige manier inzet voor de versnelde opslag-testen, enorm bedankt hiervoor! Alessia, thank you for inspiration on the experimental design of various studies in this thesis. I also appreciate that you involved me in many in-house lipid oxidation projects, which helped me to obtain a much broader view on the matter. Alessia, John and Marnix, thank you for your guidance, feedback and help, allowing me to progress my career within Unilever.

During my years as a PhD, you could find me everywhere and nowhere. I had a desk at Helix, Vlaardingen and in the last years at Hive, I was supervising students in Axis and Vlaardingen, had meetings, and doing lab-work here-and-there. So, next to my Unilever colleagues, I obviously wish to thank my WUR colleagues as well.

Harry en Jean-Paul, bedankt voor de kans die jullie me gegeven hebben om als 'spijtoptant' een $\mathrm{PhD}$ in jullie vakgroep te mogen hebben volbrengen. Ik heb me altijd enorm welkom gevoeld binnen jullie groep, ondanks dat ik weinig fysiek aanwezig was.

Marie, you became one of my co-promoters when I was already working on the project for a little over a year. Since then, I learnt a lot from you, I really appreciate how critical, fair and balanced your feedback and input are. This helped me to improve my writing skills and structuring my way of working. Next to this, you are a very kind and patient person, thanks for everything!

Peter (W.), bedankt voor je begeleiding in de eerste jaren van mijn PhD-project. Ik vond het leuk om met je te sparren, en je bracht me altijd op goede ideeën. Ook bedankt voor het medebegeleiden van studenten als ik niet in de buurt was.

Gijs, allereerst bedankt voor de heerlijke douchekoffie die ik elke ochtend om 5.30 in Tokyo geserveerd kreeg. En bedankt dat je als Unilever-stagiaire er even voor had gezorgd dat we wat zwaardere kettlebells in de sportruimte kregen. Maar zonder ongein, ik heb altijd enorm met jou kunnen lachen en leuk over wetenschap kunnen praten en soms ook lekker klagen. Bedankt voor je inspiratie.

Jianli, thank you for being such a fine student and colleague! Thank you for all the hard work you did with me, the number of mayonnaises you prepared and ASLT-samples you measured was absolutely mega. I hope to reconvene some day in the future after the pandemic is settled down internationally!

Vincent, ook jij bedankt voor de fijne samenwerking. Ik ben er stellig van overtuigd dat in jouw handen de ontwikkeling naar nog betere en vettere vet-oxidatie-methodes in goede 
handen ligt! Anja (S.) en Sten, ik vond het fijn om met jullie te sparren over vet-oxidatie in emulsies, en heb van jullie geleerd over hoe dit gaat in colloïdale systemen. En jullie enthousiasme omtrent mijn NMR-methodes waren echt motiverend! Dank daarvoor. Sylvia and Caroline, thanks for being my awesome TNI-sisters.

Jolanda, jij bedankt dat je me altijd wilde helpen als ik weer even niet snapte hoe ik dit of dat precies geregeld moest krijgen, met dit boekje bijvoorbeeld, of cursussen en congressen. I would also like to thank Bianca, Roelant and many other FCH-colleagues, who really made me feel, even though I was not always physically present, that I was part of the group. I intensely enjoyed the PhD-trips to Japan and Austria/Italy with all of you!

I would also like to thank several people on the other side of the road, in Helix. Elena, thank you very much for teaching me how to use the ESR, without you my fourth chapter would not have been possible. John (P.), jij bedankt voor al je technische hulp, zowel met de ESR als NMR. Yanzhang, I wish to thank you for being such a quick learner, ensuring that the developed NMR-LipOx-methods are properly used by new students.

Tatiana, Koen, Mattia, Suyeon, Ahmad, Abbas, Raquel, Morwarid, thanks for the great times I had on Fridays in Helix, it was always a pleasure to share the office with you all. I enjoyed hanging out with you whenever I had the opportunity. Netty, Brenda, Herbert, Camilla, and other BIP-colleagues, thank you for always making me feel welcome in your group, it was much appreciated!

During these years, I had the pleasure to supervise a handful of students, who I really want to thank for their contribution to the forthcoming of this thesis. Faruk, ik vond het een genot om met jou samen te mogen werken, je een beetje nonchalante enthousiasme was aanstekelijk. Gedurende die zes maanden dat je stage bij me liep heb ik je enorm zien groeien, en je hebt ook nog mooi werk afgeleverd, wat essentieel was voor hoofdstuk 6, dank hiervoor. Sophie, merci! Thank you for your great ideas and hard work in setting up the first iterations of the NMR-method in chapter 1. You also showed Ewoud how to really play table-tennis, well done! Mariyana, thank you for your hard work and spirit, I really appreciated the way you handled hardships, as things did often go differently than we hoped!

Yafei, I wish to thank you for your hard work in setting up the ESR-experiments, your independence and perseverance were much appreciated. Andries, bedankt voor je chocolade! En natuurlijk bedankt voor je inzet en topwerk dat je geleverd hebt. Zonder al jouw harde werk en goede ideeën was hoofdstuk 5 nooit zodanig van de grond gekomen, dank hiervoor! Lorenz, thank you for the nice time I had supervising you. I very much enjoyed it and appreciated the fact that we could both laugh with each other and keep each other sharp and 
honest. Thanks for your great ideas and hard work, being absolutely crucial for the method development in chapter 4. I am glad that you got a $\mathrm{PhD}$-position in $\mathrm{FCH}$, well deserved!

Gelukkig had en heb ik ook een leven buiten werk en mayonaise-analyses. Tijdens de jaren dat Unilever nog in Vlaardingen zat, woonde ik in Rotterdam, waar ik amper iemand kende. Dit was tot het moment dat ik bij Crossfit RTM ging trainen, want hier heb ik veel hele leuke mensen ontmoet, waaronder Tolga, Shane, Bob, Rebecca en Boyan, die mijn leven naast werk op sportief en sociaal vlak een enorme boost hebben geven dank hiervoor. Ik wil vooral hier Tolga en Shane uitlichten, omdat jullie tijdens mijn Rotterdam-periode echt goeie vrienden zijn geworden. Ik heb veel met jullie kunnen lachen, en vond het altijd geweldig om met jullie te trainen en zelfs hier en daar een wedstrijdje mee te pikken.

Willem, Dennis, Sjoerd en Shaun, jullie zijn al mijn vrienden sinds respectievelijk de basisschool, middelbare school en c1000-tijd. Dus ik ken jullie al voor zo'n vijftig tot zeventig procent van mijn leven, waardoor jullie een grote invloed hebben gehad op de persoon die ik nu ben en deze $\mathrm{PhD}$ toch mooi heb weten af te ronden. Bedankt dat jullie er altijd zijn om leuke dingen mee te doen en jullie ook totaal andere werk-interesses hebben, wat ervoor zorgt dat veel beter weet wat er naast mijn dagelijkse bubbel bestaat. Ik hoop dat we nog uit veel escape-rooms kunnen ontsnappen, binnen en buiten Nederland!

Mama, bedankt voor alles. Bedankt dat je altijd een luisterend oor bent mocht ik dat nodig hebben. Bedankt dat je altijd even lief bent. Bedankt dat je altijd goed voor ons gezorgd hebt, en ons hebt geleerd dat het waard is om altijd van het goede van de mens uit te gaan. Bedankt dat je nooit te veel druk op mij hebt gelegd, en me altijd lekker mijn ding liet doen, wetende dat het toch wel goed zou komen. Ook jij bedankt, Henri, voor het heel gelukkig maken van moederlief!

Papa, ook jij enorm bedankt. Bedankt voor het mij bijbrengen van een solide werk-ethiek, voor het laten zien van de praktische aanpak. Bedankt dat je me hebt geleerd dat mannen ook emotie mogen tonen. De competitieve geest die ik van jou heb mogen erven, was vroeger vaker een vloek dan een zegen, maar door de jaren heen heb ik dit weten om te draaien. Bedankt voor de passie voor voetbal die je van jongs af aan met ons gedeeld hebt, en dan vooral voor Ajax en Oranje.

Joyce, zusterlief. Jij bedankt dat je altijd lekker tegengas geeft en me altijd een spiegel voorhoudt, ik heb hierdoor veel over mezelf geleerd. Je bent een hele lieve zus, waarmee ik ook altijd enorm kan lachen. Diego, thank you for being such a great boyfriend to my sister, and for the amazing Italian food! 
Robin, broederlief. Bedankt dat je door dik en dun altijd mijn beste maatje bent. Wij hebben bijna volledig overlappende interesses, soms met verschillende intensiteiten. Hierdoor kan ik met jou echt overal over praten. Dit gaat van games, padel, boeken, politiek tot wetenschap, en het doen van een $\mathrm{PhD}$, waar jij ook ervaring mee hebt. Ilse, bedankt dat je zo'n leuke schoonzus bent, en jullie beiden bedankt dat ik nu twee geweldig (op het moment van schrijven) nichtjes heb, ja ik heb het over jullie, Luna en Noa.

Anja, last but zeker not least. Tijdens mijn hele $\mathrm{PhD}$ heb ik het geluk gehad om jou mijn vriendin te mogen noemen. Ik vond en vind het heerlijk dat ik bij jou echt mezelf kan zijn, waardoor ik altijd lekker thuis kan ontspannen om mezelf weer op te laden voor wat er weer komen gaat. Bedankt voor al je steun als ik even gestrest was. Ik hoop nog vele jaren van jouw bijzijn te mogen genieten. Ik hou van jou. 


\section{About the author}




\section{Curriculum vitae}

Donaldus Wilhelmus Hendricus (Donny) Merkx was born on the 14th of November 1989 in Nijmegen. The first ten years, he grew up in Alphen aan de Maas, after which he moved to Beuningen. He attended Pax Christi College in Druten and obtained his VWO-diploma in 2007 majoring both "Nature \& Health" and "Nature \& Technology".

In the same year, Donny started his study on Chemistry at the Radboud University in Nijmegen. In 2011, he obtained

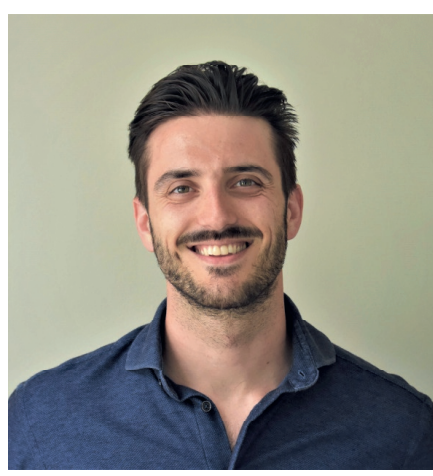
his BSc with a minor at the Synthetic Organic Chemistry department of Prof. Dr. Floris Rutjes with a thesis called "Rate constants of cycloaddition reactions on DIBAC".

Donny continued his master's majoring in Synthetic Organic Chemistry in the same group with a thesis called "Synthesis of fluorogenic cyclooctynes". He performed his internship at Janssen Pharmaceutics in Beerse (Be), where he worked on the synthesis and development of sugarlike potential anti-cancer compounds. In 2013, he graduated with the distinction 'Bene Meritum' on his master's degree.

In the same year, he started working at the R\&D department of Unilever in Vlaardingen as an NMR-analyst. In October 2016, Donny started combining his work at Unilever with a part-time $\mathrm{PhD}$ at the Wageningen University in a cooperative project called 'Tapping into Nature's Ingenuity (TNI)' where he got the opportunity to explore lipid oxidation in food emulsions in more depth, fully utilizing his NMR expertise. The results of this research are found in this thesis. In April 2021, he was appointed as a Flavour Chemistry Scientist within Unilever (now moved to Wageningen). 


\section{List of publications}

This thesis:

Merkx, D. W. H., Hong, G. T. S., Ermacora, A., \& van Duynhoven, J. P. M. (2018). Rapid Quantitative Profiling of Lipid Oxidation Products in a Food Emulsion by ${ }^{1} \mathrm{H}$ NMR. Analytical Chemistry, 90(7), 4863-4870.

Merkx, D. W. H., Delić, F., Wierenga, P. A., Hennebelle, M., \& van Duynhoven, J. P. M. (2019). ${ }^{31} \mathrm{P}$ NMR assessment of the phosvitin-iron complex in mayonnaise. Magnetic Resonance in Chemistry, 57(9), 540-547.

Merkx, D. W. H., Plankensteiner, L., Yu, Y., Wierenga, P. A., Hennebelle, M., \& van Duynhoven, J. P. M. (2021). Evaluation of PBN spin-trapped radicals as early markers of lipid oxidation in mayonnaise. Food Chemistry, 334, 127578.

Merkx, D. W. H., Swager, A., van Velzen, E. J. J., van Duynhoven, J. P. M., \& Hennebelle, M. (2021). Quantitative and Predictive Modelling of Lipid Oxidation in Mayonnaise. Antioxidants, 10(2), 287.

Merkx, D. W. H., Boerkamp, V. J. P., Hennebelle, M., \& van Duynhoven, J. P. M., Quantitative ${ }^{1} \mathrm{H}$ NMR analysis of food lipid hydroperoxides and aldehydes. In S.S. Anderson (Ed.), Multidimensional characterization of dietary lipids, New York City: Springer, to be published.

Merkx, D. W. H. ${ }^{1}$, Boerkamp, V. J. P. ${ }^{1}$, Wang, J., Hennebelle, M., \& van Duynhoven, J. P. M. Lipid epoxide quantification in a food emulsion by ${ }^{1} \mathrm{H}-{ }^{13} \mathrm{C}$ HSQC NMR spectroscopy, to be submitted.

${ }^{1}$ Equal contribution 
Other publications:

Debets, M. F., Prins, J. S., Merkx, D. W. H., van Berkel, S. S., van Delft, F. L., van Hest, J. C. M., \& Rutjes, F. P. J. T. (2014). Synthesis of DIBAC analogues with excellent SPAAC rate constants. Organic \& Biomolecular Chemistry, 12(27), 5031-5037.

van Erven, G., de Visser, R., Merkx, D. W. H., Strolenberg, W., de Gijsel, P., Gruppen, H., \& Kabel, M. A. (2017). Quantification of Lignin and Its Structural Features in Plant Biomass Using ${ }^{13} \mathrm{C}$ Lignin as Internal Standard for Pyrolysis-GC-SIM-MS. Analytical Chemistry, 89(20), 10907-10916.

Merkx, D. W. H., Westphal, Y., van Velzen, E. J. J., Thakoer, K. V., de Roo, N., \& van Duynhoven, J. P. M. (2018). Quantification of food polysaccharide mixtures by ${ }^{1} \mathrm{H}$ NMR. Carbohydrate Polymers, 179, 379-385.

Yang, S., Verhoeff, A. A., Merkx, D. W. H., van Duynhoven, J. P. M., \& Hohlbein, J. (2020). Quantitative Spatiotemporal Mapping of Lipid and Protein Oxidation in Mayonnaise. Antioxidants, 9(12), 1278.

Pandeirada, C. O., Merkx, D. W. H., Janssen, H.-G., Westphal, Y., \& Schols, H. A. (2021). TEMPO/NaClO$/ 2 / \mathrm{NaOCl}$ oxidation of arabinoxylans. Carbohydrate Polymers, 259, 117781.

Merkx, D. W. H., du Chatinier, W., El-Ouagmiri, M., van Dalen, G., Kalathil, R., \& Duchateau, G.S.M.J.E. Multivariate characterization of Mulberry extracts for alphaglucosidase inhibition, to be submitted. 


\section{Overview of Completed Training Activities}

\section{Discipline specific activities}

\begin{tabular}{lll}
\hline Courses & & \\
\hline Food Chemistry Analysis & VLAG, Wageningen, NL & $2017^{\mathrm{a}}$ \\
Reaction kinetics in Food Sciences & VLAG, Wageningen, NL & 2018 \\
Conferences & & \\
\hline Eurofed Lipids & EFL, Uppsala, Sweden & $2017^{\mathrm{b}}$ \\
PANIC & CHI, Hilton Head Island SC, USA & $2017^{\mathrm{b}}$ \\
FAST 2017 & TI COAST, Veldhoven, NL & $2017^{\mathrm{b}}$ \\
Pickering emulsion conference & FPE/VLAG, Wageningen, NL & $2018^{\mathrm{b}}$ \\
MR in Food Science & Ampere, Rennes, France & $2018^{\mathrm{a}}$ \\
Int. Symp. On Lipid Oxidation \& Antioxidants & EFL, Graz, Austria & $2018^{\mathrm{b}}$ \\
NMR Discussion Group meeting & NMRDG, Terneuzen, NL & $2019^{\mathrm{b}}$ \\
Int. Symp. On Lipid Oxidation \& Antioxidants & EFL, online & $2020^{\mathrm{b}}$
\end{tabular}

\section{General courses}

\begin{tabular}{lll}
\hline Scientific Publishing & WGS, Wageningen, NL & 2017 \\
Scientific Writing & WGS, Wageningen, NL & 2017 \\
Presenting with impact & WGS, Wageningen, NL & 2018 \\
Effective behaviour in your prof. surroundings & WGS, Wageningen, NL & 2017 \\
Introduction to R & VLAG, Wageningen, NL & 2020 \\
Brain training & WGS, Wageningen, NL & 2019 \\
Applied statistics & VLAG, Wageningen, NL & 2020 \\
& & \\
Other activities & & \\
\hline Preparation of research proposal & FCH, Wageningen & 2016 \\
PhD study tour to Japan & FCH, Japan & $2016^{\mathrm{a}, \mathrm{b}}$ \\
PhD study tour to Italy/Austria & FCH, Italy \& Austria & $2018^{\mathrm{a}, \mathrm{b}}$ \\
Scientific department meetings & FCH, Wageningen, NL & $2016-2021$ \\
Scientific department meetings & Unilever, Vlaardingen/Wageningen, NL & $2016-2021$ \\
\hline
\end{tabular}
${ }^{\mathrm{a}}$ poster presentation, ${ }^{\mathrm{b}}$ oral presentation

Abbreviations: VLAG, Graduate School for Advanced studies in Food Technology, Agrobiotechnology, Nutrition and Health Sciences; EFL, European Federation for the Science and Technology of Lipids; CHI, Cambridge Healthtech Institute; TI COAST, Top Institute COmprehensive Analytical Science and Technology; FPE, Food Process \& Engineering; NMRDG. Nuclear Magnetic Resonance Discussion Group; PANIC, Practical Applications of NMR in Industry Conference, WGS, Wageningen Graduate School; FCH, Food CHemistry. NL, the Netherlands. SC, South Carolin 
The work presented in this thesis was performed at the Unilever R\&D laboratories in Vlaardingen and Wageningen, the Netherlands, and at the Laboratories of Food Chemistry and Biophysics of Wageningen University \& Research, the Netherlands

This work was part of the project Tapping into Nature's Ingenuity - Natural Oxidation Control (TNI-NOC), which was (partly) financed by Unilever.

Cover design by Remco Wetzels

Printed by ProefschriftMaken

Edition: 250 copies

Donny Merkx, 2021 

